\title{
Physics of ULIRGs with MUSE and ALMA: The PUMA project
}

\section{Properties of the survey and first MUSE data results}

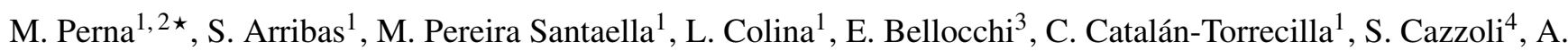 \\ Crespo Gómez ${ }^{11}$, R. Maiolind $\underline{5677}$, J. Piqueras Lópe $2^{11}$, and B. Rodríguez del Pind ${ }^{1}$
}

${ }^{1}$ Centro de Astrobiología, (CAB, CSIC-INTA), Departamento de Astrofísica, Cra. de Ajalvir Km. 4, 28850 - Torrejón de Ardoz, Madrid, Spain

2 INAF - Osservatorio Astrofisico di Arcetri, Largo Enrico Fermi 5, I-50125 Firenze, Italy

3 Centro de Astrobiología (CSIC-INTA), ESAC Campus, 28692 Villanueva de la Cañada, Madrid, Spain

4 IAA - Instituto de Astrofísica de Andalucía (CSIC), Apdo. 3004, 18008, Granada, Spain

5 University of Cambridge, Cavendish Laboratory, Cambridge CB3 OHE, UK

6 University of Cambridge, Kavli Institute for Cosmology, Cambridge CB3 OHE, UK

7 Department of Physics and Astronomy, University College London, Gower Street, London WC1E 6BT, UK

Received September 15, 1996; accepted March 16, 1997

\section{ABSTRACT}

Context. Ultraluminous infrared galaxies (ULIRGs) are characterised by extreme starburst (SB) and active galactic nucleus (AGN) activity, and are therefore ideal laboratories for studying the outflow phenomena and their feedback effects. We have recently started a project called Physics of ULIRGs with MUSE and ALMA (PUMA), which is a survey of 25 nearby $(z<0.165)$ ULIRGs observed with the integral field spectrograph MUSE and the interferometer ALMA. This sample includes systems with both AGN and SB nuclear activity in the pre- and post-coalescence phases of major mergers.

Aims. The main goals of the project are (i) to study the prevalence of (ionised, neutral, and molecular) outflows as a function of the galaxy properties, (ii) to constrain the driving mechanisms of the outflows (e.g. distinguish between SB and AGN winds), and (iii) to identify and characterise feedback effects on the host galaxy. In this first paper, we present details on the sample selection, MUSE observations, and data reduction, and derive first high-level data products.

Methods. MUSE data cubes were analysed to study the dynamical status of each of the 21 ULIRGs observed so far, taking the stellar kinematics and the morphological properties inferred from MUSE narrow-band images into account. We also located the ULIRG nuclei, taking advantage of near-infrared (HST) and millimeter (ALMA) data, and studied their optical spectra to infer (i) the ionisation state through standard optical line ratio diagnostics, and (ii) outflows in both atomic ionised ([O III], $\mathrm{H} \alpha$ ) and neutral (Na ID) gas.

Results. We show that the morphological and stellar kinematic classifications are consistent: post-coalescence systems are more likely associated with ordered motions, while interacting (binary) systems are dominated by non-ordered and streaming motions. We also find broad and asymmetric [O III] and Na ID profiles in almost all nuclear spectra, with line widths in the range [300 - 2000] $\mathrm{km} / \mathrm{s}$, possibly associated with AGN- and SB-driven winds. This result reinforces previous findings that indicated that outflows are ubiquitous during the pre- and post-coalescence phases of major mergers.

Key words. Galaxies:active - Galaxies: starburst - Galaxies: ISM - Galaxies: interactions

\section{Introduction}

Theory and observations suggest that galaxies are open systems that evolve into a quasi-stationary state, where flows of baryons determine the growth and evolution of their stellar populations and the supermassive black hole $(\mathrm{BH})$ at their centre (see e.g. Schaye et al. 2010; Bothwell et al. 2013, Sánchez Almeida et al. 2014, Hopkins et al. 2016; Naab \& Ostriker 2017; Dekel et al. 2019). Gas accretion from the cosmic web or from merging interactions triggers star formation (SF) and activity from active galactic nuclei (AGN); in turn, the energy released by the AGN and the stars heats and pushes the surrounding interstellar medium (ISM), regulating the formation of new stars and the accretion onto the BH with feedback mechanisms (e.g. Silk 2013; Hopkins et al. 2012, 2016). The role of these outflows is believed to be crucial because they not only regulate (and

^ E-mail: mperna@cab.inta-csic.es possibly quench) SF, but also redistribute dust and metals over large distances within the galaxy, or even expel them into the circumgalactic and intergalactic media (e.g. Veilleux et al. 2005. 2020). In this scenario, the galaxy mass function (e.g. Somerville \& Davé 2015), the mass-metallicity relation (Maiolino \& Mannucci 2019), and the correlation of the black hole with spheroid mass (Kormendy \& Ho 2013) are shaped by outflows.

Although this general scenario is relatively well established, many details of the processes involved remain largely unknown. An intrinsic difficulty is associated with the fact that the selfadjusted balance of gas accretion, gas outflow, and SF and AGN activity depends on the coupling between physical processes that involve multi-phase (ionised, neutral, and molecular) gas at very different physical scales, from cosmic web structures and mergers to molecular clouds (where SF occurs) and the vicinity of the BH (Sánchez Almeida et al.|2014). 
In recent years, the study of outflows at high- $z$ has gained much interest. Because the early Universe is characterised by episodes of intense SF and AGN activity (Madau \& Dickinson 2014), models predict that powerful outflows had a key role in shaping primeval galaxies and their subsequent evolution (e.g. Ceverino et al. 2018; Pillepich et al. 2018; Davé et al. 2019). Although the observational constraints provided by current facilities are limited, they indeed suggest that galaxy-scale winds are highly prevalent at the peak of SF $(z \sim 1-3$; e.g. Genzel et al. 2014; Brusa et al. 2015; Carniani et al. 2016; Föerster-Schreiber et al. 2018; Villar Martín et al. 2020, Kakkad et al. 2020), and may be extremely powerful at very early epochs (e.g. Maiolino et al. 2012; Bischetti et al. 2019).

Local ultraluminous infrared galaxies (ULIRGs, with restframe $[8-1000 \mu \mathrm{m}] \mathrm{L}_{I R}$ in excess of $\left.10^{12} L \odot\right)$ are powered by strong SBs (Genzel et al. 1998) and/or AGN (Nardini et al. 2010), and therefore are ideal laboratories for studying the outflow phenomena. While scarce in the nearby universe, luminous star-forming galaxies (SFGs) are much more numerous at high$z$; they are responsible for $\sim 50 \%$ of the total star formation rate (SFR) density at $z \sim 2$ when their number density is a factor $~$ 800x higher than locally (e.g., Pérez-González et al. 2005; Magnelli et al. 2013, Casey et al. 2014). For high-z luminous SFGs, however, some properties may differ from those of their local counterparts. While local ULIRGs are dominated by strong interactions and major mergers, recent morphological studies have found that distant luminous SFGs appear to be a mixture of merging interacting systems and disk galaxies (e.g. Arribas et al. 2012, Kartaltepe et al. 2012, Kaviraj et al. 2013, Hung et al. 2014; Alcorn et al. 2019;: Wisnioski et al. 2018), and therefore a fraction may maintain their high observed SFRs by continuous gas accretion through cold gas flows and minor mergers (Ocvirk et al. 2008; Dekel et al. 2009). Nevertheless, local ULIRGs offer the opportunity of investigating the properties of strong outflows and their feedback effects at similar SFR levels as observed at high- $z$, but at much higher spatial resolution and signal-to-noise ratio.

In the past, ULIRGs have been the target of numerous studies, which have demonstrated the presence of ionised (e.g. Heckman et al. 1990, Colina et al. 1999, Bedregal et al. 2009, Colina et al. |2012; Westmoquette et al. |2012; Rupke \& Veilleux 2013a; Rodríguez Zaurín et al. 2013, Bellocchi et al. 2013; Arribas et al. 2014, Rich et al. 2011, 2015), neutral (e.g. Martin 2005; Rupke et al. 2005a b c , Rupke \& Veilleux 2013a, Cazzoli et al. 2016), and molecular (e.g. Feruglio et al.2010, Sturm et al. 2011; Spoon et al. 2013; Veilleux et al. 2013, Rupke \& Veilleux 2013b, Cicone et al. 2014; Emonts et al. 2018;, Pereira-Santaella et al.2018, Fluetsch et al. 2019) outflows. Most of these previous works are limited to the study of a specific gas phase, however, or have resolutions that cannot trace the outflow structure in detail.

To overcome these limitations, we have recently started a project aimed at studying the outflow phenomena in a sample of 25 representative ULIRGs, based on integral field spectroscopy (IFS) with MUSE at the Very Large Telescope (VLT) and interferometric ALMA data (PUMA: "Physics of ULIRGs with MUSE and ALMA"). The combination of the data provided by these two facilities offers the possibility of studying the multi-phase structure of the outflow at subkiloparsec (sub$\mathrm{kpc}$ ) angular resolutions over a field of view (FOV) that covers the main body of the system. The key goals of this project include i) studying the prevalence of outflows as a function of the galaxy and $\mathrm{BH}$ properties, ii) establishing the relative role of the different gas phases in gas flows, iii) constraining the driving mechanisms of the outflow (e.g. distinguish between SB- and
AGN-driven winds), and iv) identifying and characterising negative and/or positive feedback, and quantifying their effects on the host galaxy. The first such detailed study of the multi-phase galaxy-scale outflows has recently been presented for the closest ULIRG in our sample, Arp 220 (Perna et al. 2020).

In this paper, we present the MUSE data for the entire PUMA sample that are required to trace and characterise the ionised and neutral components of the outflows, while in Pereira-Santaella et al. (in prep.) we describe the associated ALMA data that we use to study the molecular phase. The current MUSE data represent a significant improvement in quality with respect to previous surveys of ULIRGs based on the use of first-generation IFS instruments such as INTEGRAL (García-Marín et al. 2009ab) and VIMOS (Arribas et al. 2008). The MUSE data presented here also provide a complementary view of outflow phenomena with respect to other MUSE surveys, for instance targeting local Seyfert galaxies (e.g. MAGNUM, Mingozzi et al. 2019; CARS, Husemann et al. 2019), whose outflows would likely originate from AGN, or galaxies with prominent nuclear rings and bars (TIMER, Gadotti et al. 2019), whose outflows would probably originate from AGN and/or SB activity triggered by secular processes.

This paper is organised as follows. In Sect.2] we describe the sample selection. Section 3 presents the MUSE observations and data reduction. In Sect. 4 we describe the main morphological properties of our ULIRGs, as inferred from MUSE optical continuum and line feature structure, and from HST near-infrared (near-IR) images. In Sect. 5] we describe the dynamical status of each ULIRG, taking into account the morphological properties and the stellar kinematics derived in this work. Section 6 presents the nuclear gas properties inferred from the analysis of MUSE spectra in terms of ionisation conditions and multi-phase (ionised and neutral) outflows. Finally, Sect. 7 summarises our conclusions. Throughout this paper, we adopt the cosmological parameters $H_{0}=70 \mathrm{~km} / \mathrm{s} / \mathrm{Mpc}, \Omega_{m}=0.3$, and $\Omega_{\Lambda}=0.7$.

\section{Sample selection}

The PUMA project is designed to investigate the main properties of outflows in a representative distance-limited $(<800 \mathrm{Mpc}$, $z<0.165)$ sample of ULIRGs. The targets are selected to cover the widest possible range in host galaxy properties, such as (i) activity class (i.e. we consider systems with both AGN and SB nuclear activity), (ii) interaction stage (from advanced interacting pairs to mergers), and (iii) $8-1000 \mu \mathrm{m}$ IR luminosity.

The parent sample is assembled from the IRAS 1 Jy Survey (Kim \& Sanders 1998), the IRAS Revised Bright Galaxy sample (Sanders et al.2003), and the Duc et al. (1997) catalogue. From these surveys, we select ULIRGs with declination between -65 and +20 degrees, and within the required distance range (i.e. $z<$ $0.165)$. We also request the targets to have mid-IR Spitzer spectroscopy so that the AGN contribution can be estimated reliably even if it is completely obscured at optical wavelengths (e.g. Veilleux et al. 2009, 2013, Spoon et al.(2013). More than $80 \%$ of the general population of ULIRGs are in close pairs or in already coalescent systems (Veilleux et al.2002). We therefore focus our sample on advanced interacting systems with nuclear projected separations smaller than $10 \mathrm{kpc}$ (i.e. systems classified as IIIb, IV, and V in the Veilleux et al. 2002 scheme). Forty-four systems fulfil all these criteria, from which we selected 23 objects that uniformly sample the parameter space discussed above. In particular, about half (12) of the systems are pre-mergers of type IIIb (interacting stage hereinafter), and the remaining targets are in the later type IV and V (merger) stages. 

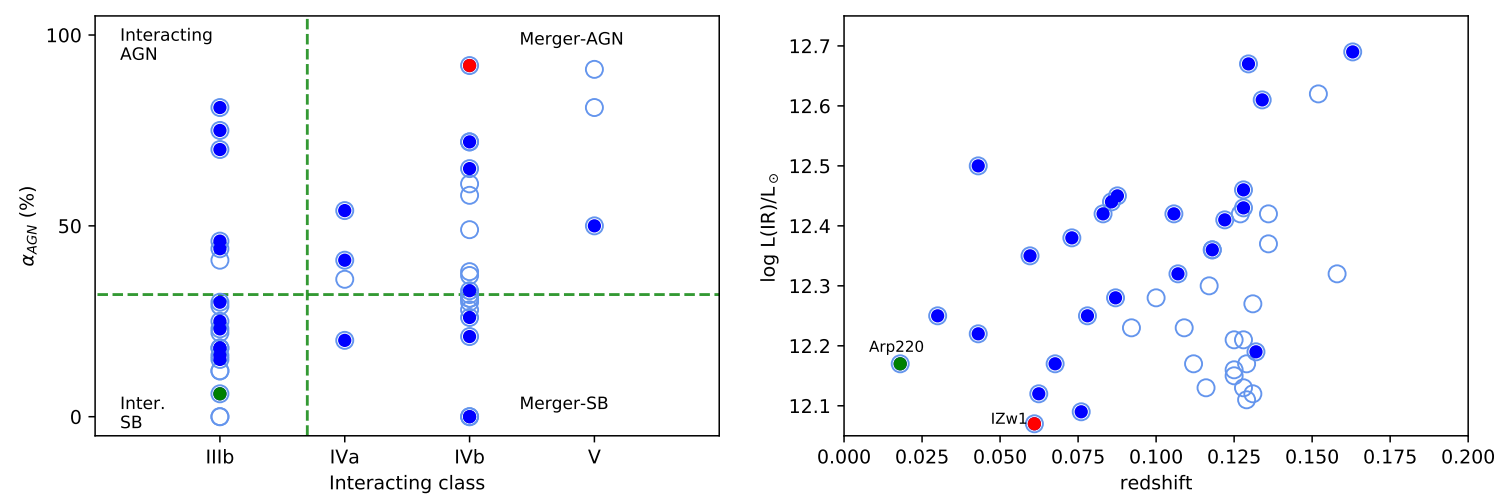

Fig. 1. Sample properties. Parent sample formed by the 44 targets that meet the selection criteria (empty and solid blue circles) and the 25 objects selected for the MUSE-ALMA sample (filled blue circles), including Arp220 (green) and IZw1 (red). See text for selection details. Left: Interacting class classification, according to the Veilleux et al. (2002) scheme, vs. contribution of the AGN to the total luminosity, according to method 6 of Veilleux et al. (2009). The vertical green line distinguishes between interacting galaxies and mergers in a more simplified classification; the horizontal line is used to separate SB and AGN targets. Right: Redshift vs. IR luminosity. For IZw1 the bolometric luminosity is considered instead of $\mathrm{L}_{I R}$.

The AGN contribution to the total bolometric luminosity, $\alpha_{A G N}$, was derived considering the 30 to $15 \mu \mathrm{m}$ flux ratio (following Veilleux et al. 2013): a median value of $32 \%$ is obtained for the selected sample of 23 targets and for the parent sample of 44 objects. We considered targets with $\alpha_{A G N}$ above this median value as significantly affected by the AGN. We also added the Arp220 system (although its declination is slightly higher) and the IZw1 galaxy (whose IR luminosity does not meet the ULIRG criterion, although $\mathrm{L}_{b o l}>10^{12} \mathrm{~L}_{\odot}$ ) to the group of 23 targets. These two systems are often considered archetypical of the local luminous SB (Arp220) and type 1 AGN (IZw1).

We finally note that all but three sources obey the wellknown radio-far-IR correlation, with a mean q-parameter of 2.37 (Yun et al. 2001). The only source with an excess in its radio emission is F13451+1232, with $q=-0.34$. This object hosts the powerful radio source $4 \mathrm{C} 12.50$, with its twinjet morphology (Lister et al. 2003). The systems 20087-0308 and F22491-1808 are instead associated with an IR excess ( $q=3.009$ and 3.025, respectively; Yun et al. 2001), possibly indicating a dust-enshrouded AGN or compact SBs.

The properties of the final sample of 25 systems we presented so far are reported in Table 1 . The left panel of Fig. 1 shows the distribution of the PUMA systems in the $\alpha_{A G N}-$ interacting class (IC) space: the horizontal line separates SB from AGN systems, while the vertical line separates interacting from merger targets. Our selected sources uniformly sample this twodimensional parameter space. In addition, the selected objects also uniformly sample the ULIRG luminosity range (right panel of Fig. 1).

\section{MUSE observations}

MUSE observations were conducted as part of our programme "Subkpc multi-phase gas structure of massive outflows in ultraluminous infrared galaxies" (ESO projects 0103.B-0391(A) and 0104.B-0151(A), PI: Arribas). The observations presented in this paper were carried out with VLT/MUSE in its adaptive-opticsassisted wide-field mode (AO-WFM; Bacon et al.|2010).

MUSE observations cover a $60^{\prime \prime} \times 60^{\prime \prime}$ FOV with a sampling of $0.2^{\prime \prime} \times 0.2^{\prime \prime}$, resulting in a dataset of $\sim 90000$ individual spectra. We used the nominal instrument setup, with a spectral coverage from 4750 to $9350 \AA$ and a mean resolution of $2.65 \AA$ (FWHM). Because we use $\mathrm{AO}$ with a sodium laser guide system, the wavelength range 5800-5970 $\AA$ is blocked to avoid contamination and saturation of the detector by sodium light. For our sources the Na ID feature is redshifted outside this wavelength range.

The requested observations were distributed in three 40minute observing blocks (OBs) with a total integration time of two hours on source for all ULIRG systems. We split the exposures in each OB into four (dithered and rotated by $90^{\circ}$ ) frames of $612 \mathrm{~s}$ each. Observations were performed with seeing 1"; the root mean square of the flux variation was $\sim 1 \%$, as measured at night by the VLT DIMM station.

Seventy percent of the MUSE targets (i.e. 17 out of the 25) were observed as part of our programs. A few sources were not observed with the entire requested time: for instance, only one frame was obtained for the source F16090-0139. Additional frames were instead obtained for 07251-02448, F110950238, and IZw1 because their first observations were classified as grade $\mathrm{C}$. In our analysis, we combined all these additional frames after verifying that including them does not affect the quality of the final data cubes negatively.

MUSE archival data exist for four out of the eight remaining targets: 13120-5453, F14378-3651, 17208-0014, and F224911808 (already presented in Fluetsch et al. 2020). These sources were observed in seeing-limited WFM and with $\sim 30$-minute exposure times. Because the seeing conditions during their observations were good, we decided to include the archival data of these four targets in our analysis. We note that seven of our selected sources are also part of the sample presented in Fluetsch et al. (2020): IZW1, 20100-1651 and 19542+1110, for which we present higher quality data, as well as $13120-5453$, F143783651, 17208-0014 and F22491-1808.

Our PUMA survey so far consists of 21 ULIRGs observed with MUSE-WFM; information about the MUSE data used in this work are collected in Table 2 At the mean distance $(\sim 400$ $\mathrm{Mpc}$ ), the MUSE spaxel scale, resolution, and FoV correspond to $0.34 \mathrm{kpc}, 1 \mathrm{kpc}$, and $\sim 100 \times 100 \mathrm{kpc}^{2}$.

\subsection{Data reduction and astrometry registration}

MUSE observations were reduced using the MUSE EsoReflex pipeline recipes (muse - 2.6.2), which provide a fully calibrated and combined MUSE data cube. In the last step of our data reduction, we identified and subtracted the residual sky contami- 
Table 1. Sources in the PUMA survey

\begin{tabular}{|c|c|c|c|c|c|c|c|c|c|c|c|c|}
\hline IRAS ID (Other) & $\begin{array}{c}\text { RA } \\
\left({ }^{h}: m{ }^{m}{ }^{s}\right)\end{array}$ & $\begin{array}{c}\text { DEC } \\
\left({ }^{\circ}:{ }^{\prime}{ }^{\prime \prime}\right)\end{array}$ & $\begin{array}{c}\mathrm{D} \\
(\mathrm{Mpc})\end{array}$ & $\begin{array}{c}\log L_{I R} \\
\left(L_{\odot}\right)\end{array}$ & $q$ & SB-AGN & $\alpha_{A G N}$ & \multicolumn{2}{|c|}{$\begin{array}{l}\text { optical } \\
\text { type }\end{array}$} & morph & IC & $\begin{array}{c}\mathrm{NS} \\
(\mathrm{kpc})\end{array}$ \\
\hline (1) & (2) & (3) & (4) & (5) & (6) & (7) & (8) & (9) & (10) & (11) & (12) & (13) \\
\hline F00091-0738 & $00: 11: 43.27$ & $-07: 22: 07.34$ & 519 & $12.27 \pm 0.03$ & 2.81 & AGN & $0.46(0.58)$ & HII & - & I & IIIb & 2.3 \\
\hline F00188-0856 & $00: 21: 26.51$ & $-08: 39: 25.99$ & 563 & $12.41 \pm 0.04$ & 2.32 & AGN & $0.50(0.35)$ & $\mathrm{LI}(\mathrm{N}) \mathrm{ER}$ & Sy 2 & M & $\mathrm{V}$ & $<0.3$ \\
\hline F00509+1225 (IZw1) & $00: 53: 34.93$ & $+12: 41: 35.94$ & 243 & $11.30 \pm 0.05^{1}$ & 2.51 & AGN & 0.92 & Sy 1 & Sy 1 & M & $\mathrm{IVb}$ & $<0.2$ \\
\hline F01572+0009 (Mrk 1014) & $01: 59: 50.25$ & $+00: 23: 40.88$ & 717 & $12.63 \pm 0.03$ & 2.01 & AGN & $0.65(0.27)$ & Sy 1 & Sy 1 & M & $\mathrm{IVb}$ & $<0.4$ \\
\hline F05189-2524 & $05: 21: 01.40$ & $-25: 21: 45.30$ & 189 & $12.17 \pm 0.01$ & 2.72 & AGN & $0.72(0.30)$ & Sy 2 & Sy 2 & M & $\mathrm{IVb}$ & $<0.1$ \\
\hline $07251-0248$ & $07: 27: 37.61$ & $-02: 54: 54.25$ & 390 & $12.41 \pm 0.04$ & 2.76 & SB & $0.30(0.39)$ & - & HII & I & $\mathrm{IIIb}$ & 1.8 \\
\hline 09022-3615 & 09:04:12.71 & $-36: 27: 01.93$ & 268 & $12.30 \pm 0.01$ & 2.21 & AGN? & $0.54(0.09)$ & HII & HII & M & IVa & $<0.1$ \\
\hline $10190+1322$ & $10: 21: 42.94$ & $+13: 06: 53.83$ & 343 & $12.05 \pm 0.03$ & 2.41 & SB & 0.17 & HII & HII & I & $\mathrm{IIIb}$ & 7.2 \\
\hline F11095-0238 & 11:12:03.38 & $-02: 54: 22.94$ & 477 & $12.28 \pm 0.03$ & 2.15 & AGN & $0.44(0.60)$ & $\mathrm{LI}(\mathrm{N}) \mathrm{ER}$ & $?$ & I & IIIb & 1.1 \\
\hline F12072-0444 & $12: 09: 45.12$ & $-05: 01: 13: 31$ & 573 & $12.40 \pm 0.03$ & 2.55 & AGN & $0.75(0.41)$ & Sy 2 & Sy 2 & I & IIIb & 2.3 \\
\hline $\mathrm{F} 12112+0305$ & $12: 13: 46.06$ & $+02: 48: 41.53$ & 329 & $12.33 \pm 0.02$ & 2.65 & SB & 0.17 & LI(N)ER & - & I & IIIb & 4.18 \\
\hline 13120-5453 (WKK 2031) & $13: 15: 06.32$ & $-55: 09: 22.82$ & 139 & $12.25 \pm 0.03$ & 2.91 & AGN & $0.33(0.01)$ & Sy 2 & Sy 2 & M & $\mathrm{IVb}$ & $<0.1$ \\
\hline $\mathrm{F} 13451+1232$ & $13: 47: 33.36$ & $+12: 17: 24.24$ & 542 & $12.31 \pm 0.04$ & -0.34 & AGN & $0.82(0.60)$ & Sy 2 & Sy 2 & I & IIIb & 4.3 \\
\hline F14348-1447 & $14: 37: 38.28$ & $-15: 00: 24.24$ & 370 & $12.35 \pm 0.02$ & 2.37 & SB & $0.17(0.04)$ & $\mathrm{LI}(\mathrm{N}) \mathrm{ER}$ & $?$ & I & IIIb & 5.45 \\
\hline F14378-3651 & $14: 40: 59.01$ & $-37: 04: 31.93$ & 302 & $12.13 \pm 0.02$ & 2.35 & AGN & $0.21(0.01)$ & Sy 2 & Sy 2 & M & IVa & $<0.1$ \\
\hline F15327+2340 (Arp220) & $15: 34: 57.22$ & $+23: 30: 11.50$ & 82 & $12.17 \pm 0.01$ & 2.61 & SB & $0.06(0.17)$ & $\mathrm{LI}(\mathrm{N}) \mathrm{ER}$ & $\mathrm{LI}(\mathrm{N}) \mathrm{ER}$ & $\mathrm{I}$ & $\mathrm{IIIb}$ & 0.37 \\
\hline F16090-0139 & $16: 11: 40.42$ & $-01: 47: 06.35$ & 592 & $12.57 \pm 0.02$ & 2.34 & SB & $0.41(0.24)$ & LI(N)ER & HII & $\mathrm{M}$ & IVa & $<0.7^{2}$ \\
\hline F16156+0146 & $16: 18: 09.37$ & $+01: 39: 21.76$ & 585 & $12.11 \pm 0.06$ & 2.18 & AGN & $0.70(0.40)$ & Sy 2 & - & I & IIIb & 8.26 \\
\hline $17208-0014$ & $17: 23: 21.96$ & $-00: 17: 00.86$ & 189 & $12.50 \pm 0.02$ & 2.70 & SB & $0.00(0.11)$ & HII & $\mathrm{LI}(\mathrm{N}) \mathrm{ER}$ & $\mathrm{M}$ & $\mathrm{IVb}$ & $<0.1$ \\
\hline F19297-0406 & $19: 32: 22.30$ & $-04: 00: 01.80$ & 376 & $12.40 \pm 0.02$ & 2.49 & SB & $0.23(0.01)$ & HII & ? & I & $\mathrm{IIIb}^{3}$ & 1.1 \\
\hline $19542+1110$ & $19: 56: 35.78$ & $+11: 19: 05.03$ & 284 & $12.12 \pm 0.04$ & 2.55 & SB & $0.26(0.04)$ & LI(N)ER & LI(N)ER & $\mathrm{M}$ & $\mathrm{IVb}$ & $<0.1$ \\
\hline 20087-0308 & $20: 11: 23.87$ & $-02: 59: 50.71$ & 463 & $12.44 \pm 0.03$ & 3.01 & SB & $0.20(0.03)$ & LI(N)ER & LI(N)ER & M & IVa & $<0.9$ \\
\hline $20100-4156$ & $20: 13: 29.56$ & $-41: 47: 35.21$ & 571 & $12.64 \pm 0.02$ & 2.49 & SB & $0.26(0.20)$ & HII & LI(N)ER & I & IIIb & 6.5 \\
\hline F20414-1651 & $20: 44: 18.16$ & $-16: 40: 16.82$ & 381 & $12.30 \pm 0.08$ & 2.47 & SB & 0.00 & HII & - & M & $\mathrm{IVb}$ & $<0.2$ \\
\hline F22491-1808 & $22: 51: 49.24$ & $-17: 52: 23.66$ & 339 & $12.19 \pm 0.03$ & 3.03 & $\mathrm{SB}$ & 0.15 & HII & HII & I & IIIb & 2.7 \\
\hline
\end{tabular}

Notes. Column (1): IRAS identification code (alternative name); (2) and (3): RA and DEC coordinates of the ULIRG nuclei from this work; for binary systems, we report the coordinates of the near-IR brightest nucleus; (4): Hubble distance (from the NED); (5): IR luminosity; (6): q radio-IR parameter (Condon et al.|1991); (7): simplified AGN-SB classification based on the information provided in columns (8) and (9); (8): fraction of AGN contribution to $L_{b o l}$ derived from the 30 to $15 \mu \mathrm{m}$ flux ratio $(f 30 / f 15)$. This ratio gives a good first-order approach to the contribution of the AGN to $\mathrm{L}_{\text {bol }}$ because it agrees to within $10-15 \%$ with the average value of the six methods considered in Veilleux et al. (2009). In order to homogenise the various calibrations of this ratio, we took the results of Veilleux et al. (2013) as reference and fit a second-order polynomial to the $\log (f 30 / f 15)$ function. The flux ratio values are from Veilleux et al. (2009.2013) and Spoon et al. (2013). Values in brackets are derived according to the method of Nardini et al. (2010), which is based on the analysis of the $5-8 \mu \mathrm{m}$ spectral range; (9-10) optical spectral type according to Veilleux et al. (2009. 2013) in column 9, and according to our classification obtained from nuclear spectra (Sect. 6) in column 10; (11): simplified morphology classes (I: interacting, M: mergers); see text for details. (12): IC according to the scheme by Veilleux et al. (2002), obtained from Veilleux et al. (2009) or derived from HST imaging; (13): Nuclear separation from/Veilleux et al. (2009), Duc et al. (1997), and Kim et al. (2013). ${ }^{1}[40-500] \mu \mathrm{m} \mathrm{IR}$ luminosity from Petric et al. (2015). This target does not meet the IR luminosity criterion for being a ULIRG (although $\mathrm{L}_{b o l}>10^{12} \mathrm{~L}_{\odot}$ ), but it has been included because it is usually considered an archetypical type 1 AGN.

${ }^{2}$ No HST images are available for F16090-0139, therefore the NS upper limit has been obtained on the basis of our ALMA images (PereiraSantaella et al., in prep).

${ }^{3}$ F19297-0406, originally associated with a single nucleus (Kim et al.|2013), is here classified as a IIIb system because two nuclei in near-IR HST and ALMA (Pereira-Santaella et al., in prep) images are detected.

nation in the final data cube using the Zurich Atmosphere Purge (ZAP) software package (Soto et al. 2016; see e.g. Sect. 3 in Perna et al. 2020).

We estimated the spatial resolution from the foreground stars in the MUSE FOV for all but Arp220 and F11095-0238, for which no stars are detected. For each bright star in the FOV, we performed a 2D Gaussian fit, and derived an estimate for the angular resolution from the FWHM of the Gaussian fit. The spatial resolution in the data cube of F11095-0238 was obtained from a 2D Gaussian fit of the broad-line region (BLR) emission of a quasi-stellar object (QSO) at $\mathrm{z} \sim 2.2$ (CXOGSG J111204.2-025415); the spectral resolution of Arp220 was taken from Perna et al. (2020). Because of the MUSE seeing-enhancer mode (and the good seeing conditions during the acquisition of seeing-limited MUSE data), we reached an overall average resolution of $\sim 0.7^{\prime \prime}$, corresponding to physical scales in the range $\sim 0.2-2 \mathrm{kpc}$ depending on the redshift of the system (Table 2).

The astrometric registration was performed using the Gaia DR2 catalogue (Gaia Collaboration 2018). We created continuum maps from the MUSE observations (see Sect. 4]and Fig. 2) where we measured the position of all the Gaia DR2 stars present in the MUSE FOV. Then, we shifted the MUSE cube astrometric solution to minimise the difference between the Gaia sky coordinates of these stars and those derived from the MUSE data. We took the proper motions of the stars as listed in the Gaia catalogue into account. For most systems, we used between 2 and 35 Gaia stars to perform this correction. For 5 systems (F051892524, F12071-0444, F13451+1232, F14348-1447, and F110950238), fewer than 2 Gaia stars were available in the MUSE FOV; therefore we used HST optical images (F814W) as reference whose astrometry was tied to Gaia DR2 using the method described above. Then, we used objects detected in both the HST and MUSE images to determine the MUSE astrometric solution. HST near-IR image (F160W) astrometry was similarly tied to Gaia DR2. We estimate an average uncertainty in the MUSE astrometric solution of $0.15^{\prime \prime}(0.7$ MUSE spaxels) based on the comparison between measured and expected coordinates of the Gaia stars. 

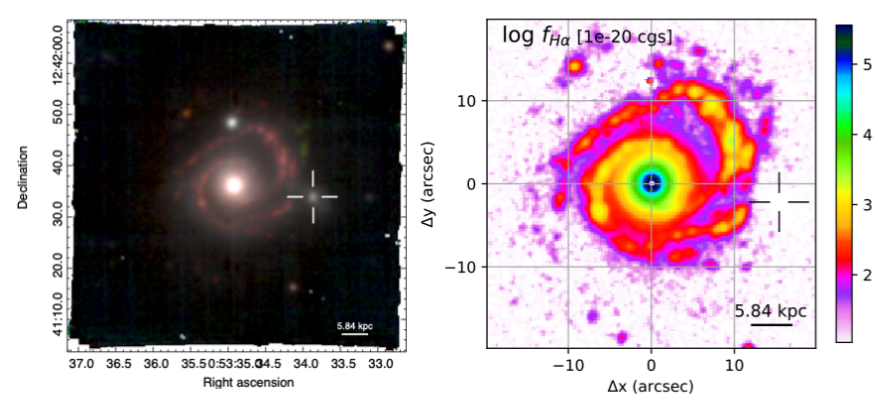

I Zw 1
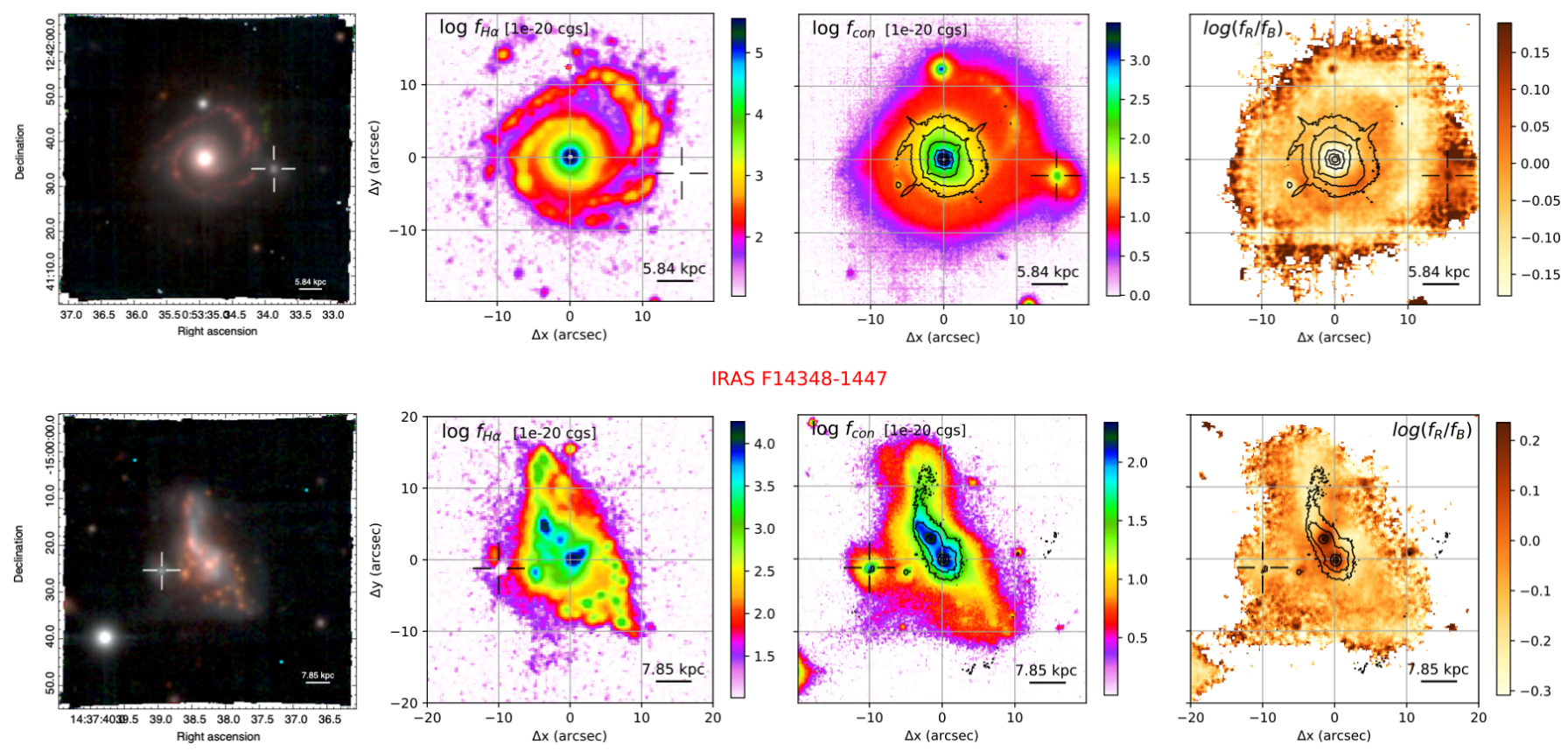

IRAS F14348-1447

Fig. 2. IZw1 (top) and F14348-1447 (bottom) MUSE images. Left column: Colour-composite optical image, showing [O III] (green), H $\alpha$ (red), and continuum emission (blue). Second column: $\mathrm{H} \alpha$ map obtained by collapsing the MUSE data cube on the emission line after subtracting the continuum using adjacent regions at shorter and longer wavelengths. Third column: Red continuum ( $8500 \AA ̊)$ image from MUSE, with contours from HST/F160W. Right column: Continuum colour map obtained from MUSE by dividing the red continuum image $(\sim 8500 \AA)$ by the blue image $(\sim 4500 \AA)$; contours from HST/F160W. See Appendix C for further details. The crosses in the panels show the detected satellites in the MUSE FOV.

\section{Stellar and ionised gas distributions}

In order to appreciate the MUSE data quality and its scientific potential, in this section we present three-colour, continuum, and emission line maps built directly from the MUSE data cubes, and briefly describe the general properties that can be inferred from them. A full characterisation of the systems, similar to the one obtained for Arp220 (Perna et al. 2020; Catalán-Torrecilla et al., in prep.), will be presented in future papers.

In particular, in this section we present the maps for two representative targets in our sample: IZw1, the type 1 AGN archetypical, hosted by an ongoing minor merger (e.g. Scharwächter et al. 2007), and the ULIRG F14348-1447, an SBdominated interacting system. Colour and flux images for the remaining targets in our sample are presented in Appendix C.

\subsection{Colour-composite maps}

Colour-composites of the two representative targets are shown in Fig. 2 (left column). These maps are built using three images derived by collapsing the MUSE data cubes along the wavelength direction. The green and red images cover the [O III $] \lambda 5007$ ([O III] hereinafter) and $\mathrm{H} \alpha$ line transitions (with underlying continuum emission), respectively. In particular, for each target, we selected the wavelength ranges covering the [O III] lines taking into account the line velocity variations observed across the MUSE FOV; the $\mathrm{H} \alpha$ maps were constructed in a similar fashion, but reducing the velocity range to minimise the contamination from the $[\mathrm{N} \mathrm{II}]$ doublet. The individual wavelength ranges used to produce composite images are reported in the figure captions in Appendix C. The blue image is instead associated with the continuum emission at bluest wavelengths. The blue, green, and red images have identical colour-bar scales and limits, and are combined to obtain an approximation to the actual relative contributions (and extensions) of [O III], $\mathrm{H} \alpha$, and stellar continuum emission in the ULIRGs.

These composites provide a first-order description of the ULIRG morphologies. Compact and regular galactic structures are observed only in three targets: IZw1 (Fig. 2, top left), F00188-0856 (Fig. C.1), and 19542+1110 (Fig. C.74). The remaining ULIRGs present irregular morphologies (e.g. elongated along the merger axis; e.g. Fig. 2, bottom left) and bright tidal tails extended over tens of kpc (e.g. Fig. C.22).

The three-colour images also allow us to compare continuum and ionised gas distribution, providing an indication of the physical processes in the ULIRGs. $\mathrm{H} \alpha$ emission generally matches the stellar continuum distributions, both in the innermost nuclear regions, tracing $\mathrm{SB}$ or AGN activity, and in the outer regions, tracing more diffuse ionised gas. Clumpy $\mathrm{H} \alpha$ emission is also detected along tidal arms. In a few targets, the [O III] dominates continuum and $\mathrm{H} \alpha$ emission, possibly indicating AGN ionisation conditions (see e.g. IZw1 nuclear and north-west regions in Fig. 2, top left).

Finally, these images provide a first-order identification of foreground stars, generally associated with bright point-like sources, and galaxies at different redshifts, showing a heterogeneous mix of morphologies, colours, and sizes (see also e.g. Perna et al. 2020). Nine ULIRGs in our sample present between one and two nearby satellites in the MUSE FOV at projected distances from $\sim 5$ to $\sim 50 \mathrm{kpc}$ (see e.g. crosses in Fig. 2), and with velocities within a few $100 \mathrm{~s} \mathrm{~km} / \mathrm{s}$ from the ULIRG systemics (Table A.1). A more detailed description of ULIRG structures and environmental properties is provided in Sect. 5.3. where we combine optical (MUSE) and near-IR (HST) imaging information with stellar kinematics results. 
Table 2. MUSE observation resolutions and exposure times

\begin{tabular}{lccc} 
IRAS ID & $\begin{array}{c}\text { resolution } \\
(" / \mathrm{kpc}) \\
(2)\end{array}$ & $\begin{array}{c}\text { seeing } \\
\left({ }^{\prime \prime}\right)\end{array}$ & $\begin{array}{c}\mathrm{t}_{\exp } \\
(\mathrm{h})\end{array}$ \\
(1) & $0.70 / 1.60$ & 0.7 & 0.68 \\
\hline F00188-0856 & $0.70 / 0.80$ & 0.8 & 2.72 \\
IZw1 & $0.70 / 2.00$ & 0.8 & 2.04 \\
F01572+0009 & $0.60 / 0.50$ & 0.7 & 2.04 \\
F05189-2524 & 0.60 \\
$07251-0248$ & $0.55 / 0.45$ & 0.7 & 2.55 \\
09022-3615 & $0.60 / 0.70$ & 0.9 & 2.04 \\
$10190+1322$ & $0.7 / 1.00$ & 0.8 & 2.04 \\
F11095-0238 & $0.75 / 1.50$ & 0.9 & 2.72 \\
F12072-0444 & $0.80 / 1.90$ & 0.8 & 0.68 \\
13120-5453* & $0.90 / 0.60$ & 0.9 & 0.52 \\
F13451+1232 & $0.75 / 1.70$ & 0.9 & 2.04 \\
F14348-1447 & $0.70 / 1.10$ & 1.0 & 0.68 \\
F14378-3651* & $0.90 / 1.10$ & 1.0 & 0.47 \\
Arp220 & $0.55 / 0.20$ & 1.0 & 0.65 \\
F16090-0139 & $0.70 / 1.70$ & 1.1 & 0.17 \\
17208-0014* & $0.55 / 0.45$ & 0.4 & 0.39 \\
F19297-0406 & $0.65 / 1.05$ & 1.0 & 2.04 \\
19542+1110 & $0.70 / 0.80$ & 0.7 & 2.04 \\
20087-0308 & $0.70 / 1.40$ & 1.1 & 2.04 \\
20100-4156 & $0.60 / 1.40$ & 0.8 & 2.04 \\
F22491-1808* & $0.50 / 0.70$ & 0.4 & 0.41 \\
\hline
\end{tabular}

Notes. Column (1): Target name. (2): Measured angular resolution at $7000 \AA$, and physical scale at the redshift of the detected target. (3): Mean DIMM seeing during the time of the observation. (4): Total integration time.

*: Seeing-limited MUSE observations from the programs 0102.B0617(A) (13120-5453 and F14378-3651) and 0101.B-0368(B) (172080014 and F22491-1808).

\subsection{Ionised gas and continuum emission maps}

In Fig. 2 we also display the $\mathrm{H} \alpha$ maps (second column), the red continuum emission (third column), and the continuum colour maps (right column) for IZw1 and F14348-1447. In the last two columns, we show the HST/F160W emission with black contours. The $\mathrm{H} \alpha$ maps are obtained by collapsing the MUSE data cube on the emission line after subtracting the continuum using adjacent regions at shorter and longer wavelengths; the red image is associated with the continuum emission at reddest wavelengths, but avoiding the range $9000-9300 \AA$ (observer frame), which is sometimes affected by poor background subtraction; the continuum-colour maps are instead built by dividing the red image by a blue one, derived by collapsing the MUSE data cube at wavelengths of $\sim 4500 \AA$. These maps are centred on the position of the ULIRG nuclei, corresponding to the peak emission in the near-IR HST images (black contours in Fig. 2). For F143481447 (and other systems with double nuclei), the zero-position corresponds to the brightest nucleus in the HST/F160W image. $\mathrm{H} \alpha$ and continuum emission maps for the entire sample are reported in Appendix C

The inspection of these images allows us a first identification of bright star-forming clumps in the outermost regions, associated with strong $\mathrm{H} \alpha$ and blue continuum emissions (e.g. along the IZw1 galactic arms in Fig. 2), or old stellar populations with faint (or absent) $\mathrm{H} \alpha$ and red spectra (e.g. in the IZw1 west satellite). Tidal tails in our sample show both red and blue continuum emission (see e.g. Fig. C.22); blue tails are generally associated with $\mathrm{H} \alpha$ emission, suggesting a certain sign of recent SF (e.g. Yuan et al. 2018).

The innermost regions of ULIRGs usually show strong $\mathrm{H} \alpha$ emission and red continuum, also associated with bright
near-IR emission (black contours) in almost all our targets. This is probably due to the severe channelling of gas and dust during the merger process, which is capable of triggering intense AGN and SB activities (responsible for the $\mathrm{H} \alpha$ nuclear emission). An imperfect match of the MUSE optical and HST near-IR peak positions is instead observed for 09022-3615, 17208-0014, and the W nucleus of F22491-1808: these discrepancies could be explained considering an even higher nuclear extinction, associated with cold circumnuclear material (Pereira-Santaella et al., in prep.) or with dust lanes across the galaxy cores (see e.g Fig. C.63). Blue nuclear spectra are observed in IZw1 (Fig. 2, top right) and F01572+0009 (Fig. C.9), the two Seyfert 1 galaxies in our sample.

In Appendix C we also report [O III] and [S II] $\lambda \lambda 6716,31$ maps for all targets. $\mathrm{H} \alpha$ emission is generally brighter and more extended than [O III]: the former is generally detected in plumes, filaments, and extended arcs (e.g. Fig. C.32) as well as in compact clumps (e.g. Fig. C.23, possibly indicating shocks (e.g. when bright [S II] is also detected) or SF activity.

Overall, ionised gas and continuum emission maps display a great richness in spatial detail at different spatial scales. In all cases, they reveal different levels of galaxy interaction and strong nuclear activity, in line with their ULIRG nature.

\section{Dynamical status}

\subsection{Stellar feature modelling}

We used the penalised pixel-fitting routines (pPXF; Cappellari \& Emsellem 2004, Cappellari 2017) to extract the stellar kinematics. We made use of the Indo-U.S. Coudé Feed Spectral Library (Valdes et al. 2004) as stellar spectral templates to model the stellar continuum emission and absorption line systems. The models, with a spectral resolution of $1.35 \AA$, were broadened to the (wavelength-dependent) spectral resolution of the MUSE data $(\sim 2.6-2.9 \AA)$ before the fitting process (see e.g. Husser et al. 2016). pPXF fits were performed on binned spaxels using a Voronoi tessellation (Cappellari \& Copin 2003) to achieve a minimum signal-to-noise ratio $\mathrm{S} / \mathrm{N}>16$ per wavelength channel on the continuum in the vicinity of the MgIb transitions. We note, however, that in the innermost nuclear regions, a large fraction of the original spaxels remains unbinned because of the high quality of the MUSE data.

The entire wavelength range covered with MUSE (i.e. 4700$9300 \AA$, observer frame) was used in our analysis to model the stellar component and recover the stellar kinematics after masking all optical emission lines detected in the data cubes. Because broad emission lines alter the local continuum and the stellar absorption profiles, causing poor pPXF fits, we used wider masks in the spatial regions where broad profiles are detected; for the two Sy 1 sources, much broader masks were used for the BLR lines in the innermost nuclear regions. In addition, we masked the resonant $\mathrm{Na}$ ID transitions because both stellar and interstellar absorption can cause these lines. Finally, we excluded a narrow wavelength region at $\sim 7630 \AA$ (observer frame) from the analysis, which is associated with strong sky-subtraction residuals, and the region $5800-5970 \AA$, which is blocked by a filter to avoid contamination from the AO lasers (see Sect. 3 ).

During the fitting procedure, we used fourth-order multiplicative Legendre polynomials to match the overall spectral shape of the data. These polynomials are generally used instead of an extinction law, which was found to produce poorer fits to the stellar continuum, and allow us to correct for small inaccuracies in the flux calibration (see e.g. Belfiore et al. 2019). 


\subsection{Stellar velocity fields and velocity dispersion maps}

The pPXF best-fit results were used to derive the systemic redshift of the ULIRGs. In particular, for the sources with one nucleus, we derived the zero-velocity by measuring the stellar kinematics at the position of the nucleus; when two nuclei are detected instead, we set the $V_{*}=0 \mathrm{~km} / \mathrm{s}$ measuring the stellar kinematics at the position of the brightest nucleus in the red wavelengths. The target 09022-3615 does not show well-defined nuclear regions in the available HST images (covering the restframe UV and optical regimes); therefore, the nucleus of this target was located on the basis of ALMA millimeter continuum and $\mathrm{CO}(2-1)$ emission line maps (Pereira-Santaella et al., in prep.). Finally, for the two Sy 1 sources with strong continuum and BLR emission in the central regions (preventing the detection of stellar features), the systemic redshifts were chosen to obtain a symmetric stellar velocity gradient along their major axis, taking advantage of their well-defined rotational patterns (see below). All spectroscopic redshifts are reported in Table A.1. together with the coordinates of the nuclei. In this table, we also report the position and spectroscopic (pPXF) redshifts of the ULIRG nearby companions detected in the MUSE data cubes.

Figure 3 shows the IZw1 (top) and F14348-1447 (bottom) stellar kinematic maps derived from $\mathrm{pPxF}$ analysis. In Appendix C we report the stellar kinematic maps for all ULIRGs in our sample. An ordered disk-like rotation can be observed in IZw1, as well as in another ten ULIRGs in our sample (e.g. Figs. C.3 and C.15). These galaxies show a well-defined velocity gradient axis, with line-of-sight velocity amplitudes of $\sim \pm 100$ $\mathrm{km} / \mathrm{s}$. They also present regular velocity dispersion configurations, with $\sigma_{*}$ of a few $100 \mathrm{~s} \mathrm{~km} / \mathrm{s}$ in the nuclear regions, and $\sigma_{*}$ close to the MUSE spectral resolution outside. However, signatures of tidal interactions are present in many of them, especially in the outermost regions (e.g. Figs. C.11 and C.43).

F14348-1447 (Fig. 3, bottom) shows a kinematic axis following the ULIRG elongated structure, on scales of $\sim 30 \mathrm{kpc}$ and with amplitudes up to $\sim \pm 150 \mathrm{~km} / \mathrm{s}$. The velocity dispersion map is quite irregular, although higher $\sigma_{*}(\sim 120 \mathrm{~km} / \mathrm{s})$ can be found at the location of its nuclei. 20087-0308 (Fig. C.78) and F22491-1808 (Fig. C.87) show very similar characteristics. Their kinematics are therefore reasonably strongly affected by the interaction of the merging galaxies.

Different configurations can instead be observed in 09022 3615 (Fig. C.24), F12072-0444 (Fig. C.38), F13451+1232 (Fig. C.47), and 20100-4156 (Fig. C.82): they show clear evidence of non-rotational motions, without preferential velocity gradient axes and with high $\sigma_{*}(>100 \mathrm{~km} / \mathrm{s})$ over the entire systems. For these ULIRGs, the stellar kinematics are therefore reasonably dominated by tidal forces as well.

Finally, F11095-0238 (Fig. C.33) and F16090-0139 (Fig. C.61) do not show clear kinematic patterns. F11095-0238 pPXF maps could suggest a nearly face-on orientation because of the small velocity amplitudes across the system and the relatively higher $\sigma_{*}$ in the innermost nuclear regions. The unconstrained kinematics in F16090-0139 are instead probably due to its very short exposure time (and low $\mathrm{S} / \mathrm{N}$ ).

We stress here that more regular kinematics might be present on subkpc scales in the innermost nuclear regions and in the vicinity of the nuclei of binary systems (see e.g. Medling et al. 2014), even when large-scale motions are irregular or are dominated by streams. For instance, Pereira-Santaella et al. (2018) reported circumnuclear molecular disks around the F14348-1447 SW and NE nuclei. More detailed investigation of subkpc motions in individual systems will be presented in a forthcoming
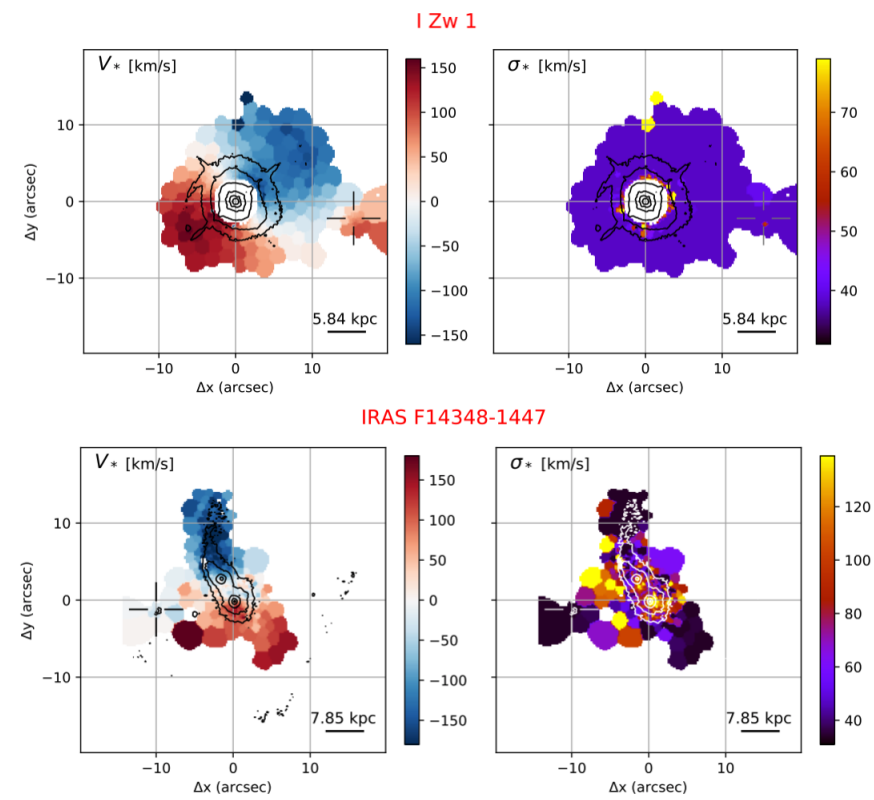

Fig. 3. pPXF stellar line-of-sight velocity (left) and velocity dispersion maps (right) for IZw1 (top) and F14348-1447 (bottom); $\sigma_{*}$ are not corrected for instrumental broadening. Contours from HST/F160W images, starting from $3 \sigma$ levels, equally spaced in steps of 0.5 dex. The IZw 1 central pixels have been masked because of the strong continuum and BLR emission, which prevents the detection of stellar absorption features.

paper; here we focus on the main (kpc-scale) stellar kinematic features.

\subsection{Stellar velocity versus morphological classification}

Table 3 summarises the morphological and stellar kinematics information we collected so far for our ULIRGs. They are based on the current MUSE colour and flux maps, HST/F160W imaging, and pPXF stellar kinematic results. For a more immediate visualisation, we also report in Figs. 4 and 5 the MUSE $\mathrm{H} \alpha$ and stellar velocity maps that we also present in Appendix C within a simplified version of the $\alpha_{A G N}-\mathrm{IC}$ diagram. These properties allow us to revise the morphological classification (with respect to the one used for the selection process in Sect.2), and confirm consistency with the derived stellar kinematics.

According to Table 3, the observed 21 ULIRGs can be divided into four different groups, taking their IC and the main stellar kinematic features into account. The first group contains 8 sources characterised by ordered disk-like rotations and a merger classification, with a single nucleus and small nearby companions (F00188-0856, IZw1, F01572+0009, F05189-2524, 13120-5453, F14378-3651, 17208-0014, and 19542+1110). Almost all of them show additional detached (non-rotational) velocity structures in the outermost regions, possibly due to tidal tails.

The second group contains three systems, characterised by ordered disk-like motions and an interacting classification: 07251-0248, 10190+1322, and Arp220. 07251-0248 shows a disk-like rotation pattern with a kinematic centre close to the two nuclei, separated by $1.82 \mathrm{kpc}$, and a strong extended tidal tail. 10190+1322 shows two overlapping disks centred at the positions of the two nuclei, separated by $\sim 7 \mathrm{kpc}$. Finally, Arp220 show a disturbed kpc-scale disk in the innermost nuclear regions (see also Fig. 3 in Perna et al. 2020). 


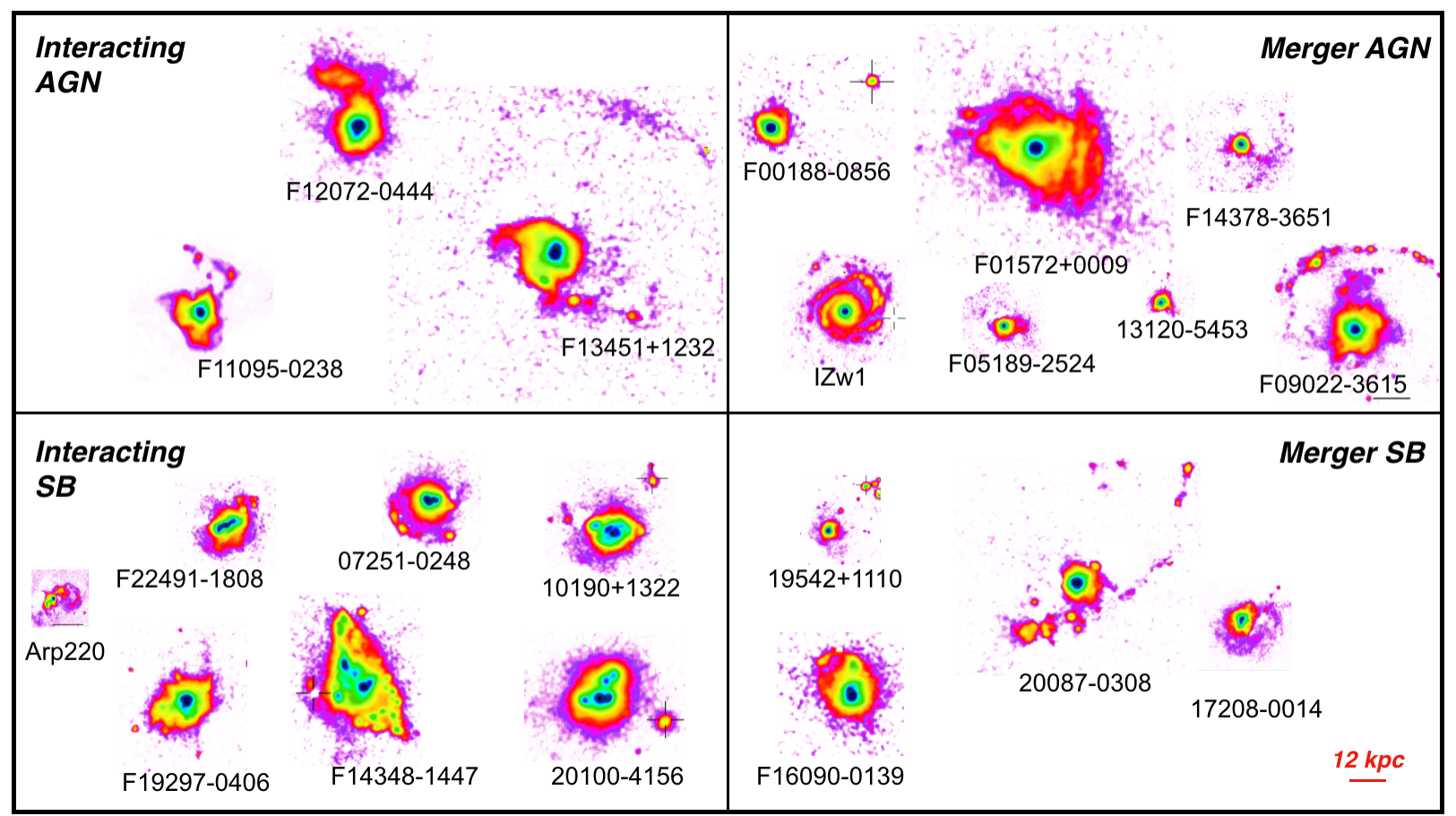

Fig. 4. MUSE H $\alpha$ maps for all 21 ULIRGs of the PUMA sample. All maps are taken from the images shown in Appendix C The galaxies are displayed in a simplified version of the $\alpha_{A G N}$ - IC plane shown in Fig. 1. with the same physical scale; north is up.

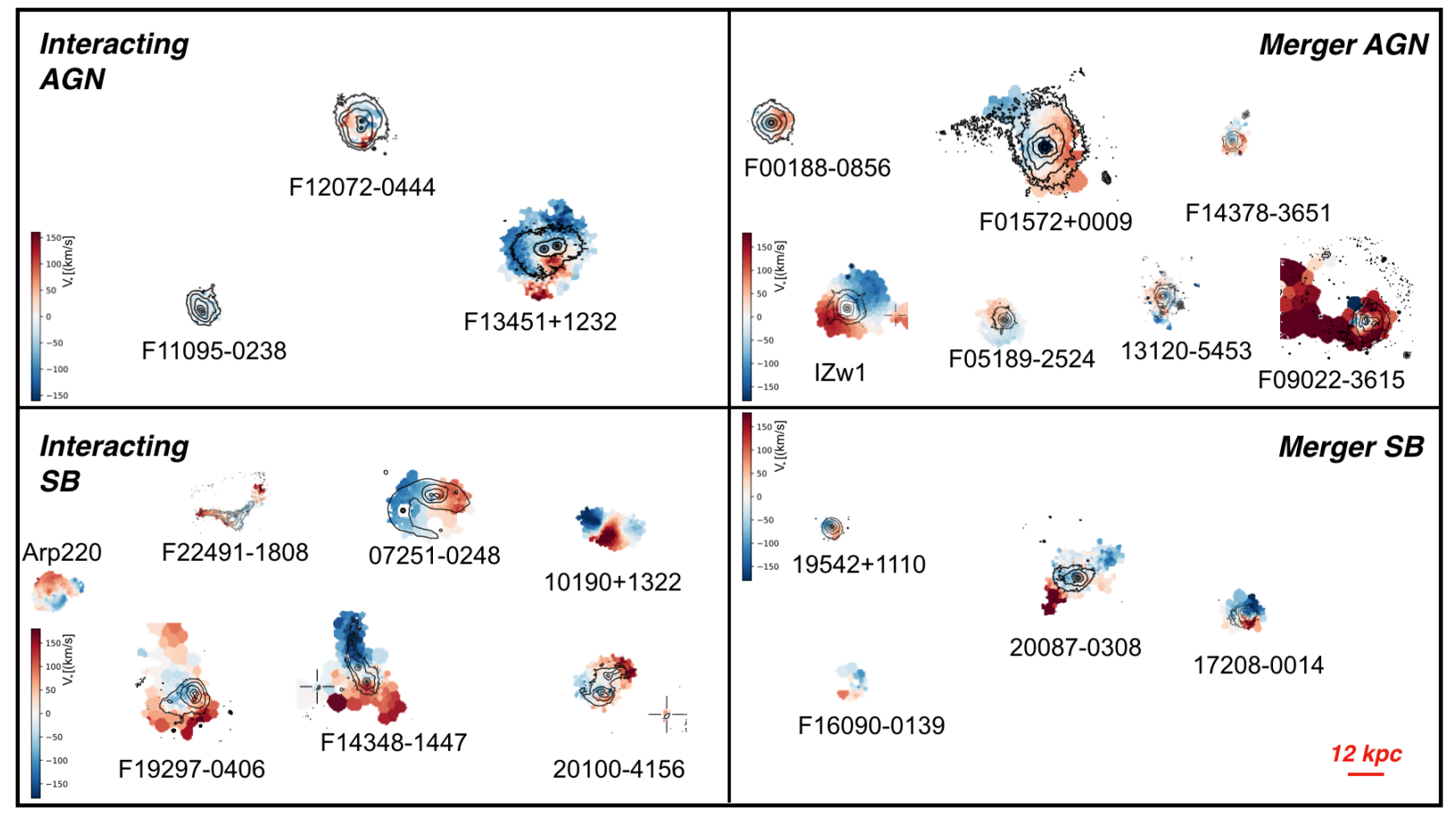

Fig. 5. MUSE stellar velocity maps for all 21 ULIRGs of the PUMA sample taken from the images shown in Appendix C The galaxies are displayed in a simplified version of the $\alpha_{A G N}$ IC plane shown in Fig. 1. with the same physical scale; north is up.

The third group contains three ULIRGs, mainly characterised by large-scale streaming motions along the axes connecting their nuclei (F14348-1447 and F22491-1808), or along the strong and extended tails (20087-0308). F14348-1447 and F22491-1808 are interacting, while 20087-0308 is classified as a merger system.

Finally, the last group contains seven systems, mainly characterised by non-ordered stellar motions: 09022-3615, F110950238, F12072-0444, F13451+1232, F16090-0139, F19297-
0406, and 20100-4156. Two of them have a merger classification (09022-3615 and F16090-0139), and the remaining sources are interacting.

Two sources deserve a special mention: 09022-3615 and F19297-0406. HST/F814W and HST/F225W images of 090223615 show two main regions of intense SF in the central part of the system, with a few bright compact ( $\lesssim 0.1^{\prime \prime}$, i.e. $\left.150 \mathrm{pc}\right)$ clumps at the position of the two peaks in the MUSE continuum image (Fig. C.22). These findings might suggest two SB 
Table 3. Morphological and kinematic classification

\begin{tabular}{lllcc}
$\begin{array}{l}\text { IRAS ID } \\
\text { (1) }\end{array}$ & $\begin{array}{l}\text { overall stellar kinematics } \\
\text { (2) }\end{array}$ & $\begin{array}{l}\text { main morphological features } \\
(3)\end{array}$ & $\begin{array}{c}\mathrm{N}_{\text {comp }} \\
(4)\end{array}$ & $\begin{array}{c}\text { IC } \\
(5)\end{array}$ \\
\hline F00188-0856 & ordered motions & 1 nucleus & 1 & $\mathrm{M}$ \\
IZw1 & ordered motions & 1 nucleus, spiral arms, [O III] clumps at kpc-scales & 1 & $\mathrm{M}$ \\
F01572+0009 & ordered motions & 1 nucleus, tails, extended H $\alpha$ & 1 & $\mathrm{M}$ \\
F05189-2524 & ordered motions & 1 nucleus, several tails, extended H $\alpha$ & 0 & $\mathrm{M}$ \\
$07251-0248$ & ordered motions & 2 nuclei, ring-like shape, H $\alpha$ clumps at kpc-scales & 0 & $\mathrm{I}$ \\
$09022-3615$ & non-ordered motions & 1 nucleus, tail, H $\alpha$ clumps at kpc-scales & 0 & $\mathrm{M}$ \\
$10190+1322$ & ordered motions & 2 nuclei and overlapping disks, H $\alpha$ clumps at kpc-scales & 2 & $\mathrm{I}$ \\
F11095-0238 & non-ordered motions & 2 nuclei, tails, extended H $\alpha$ at kpc-scales & 0 & $\mathrm{I}$ \\
F12072-0444 & non-ordered motions & 2 nuclei, tails, diffuse H $\alpha$ at kpc-scales & 2 & $\mathrm{I}$ \\
$13120-5453$ & ordered motions & 1 nucleus, extended structures and tails & 0 & $\mathrm{M}$ \\
F13451+1232 & non-ordered motions & 2 nuclei, tails, H $\alpha$ clumps at kpc-scales & 0 & $\mathrm{I}$ \\
F14348-1447 & large-scale streaming motions & 2 nuclei, H $\alpha$ clumps at kpc-scales & 1 & $\mathrm{I}$ \\
F14378-3651 & ordered motions & 1 nucleus, tails, and extended H $\alpha$ shell & 1 & $\mathrm{M}$ \\
Arp220 & ordered motions & 2 nuclei, streaming motions, H $\alpha$ shells & 0 & $\mathrm{I}$ \\
F16090-0139 & non-ordered motions & 1 nucleus, plume, H $\alpha$ bubble & 0 & $\mathrm{M}$ \\
$17208-0014$ & ordered motions & 1 nucleus, complex structures and kpc-scale tails & 0 & $\mathrm{M}$ \\
F19297-0406 & non-ordered motions & 1 nucleus, tails & 0 & $\mathrm{I}$ \\
$19542+1110$ & ordered motions & 1 nucleus, H $\alpha$ clumps at kpc-scales & 1 & $\mathrm{M}$ \\
$20087-0308$ & large-scale streaming motions & 1 nucleus, tails, H $\alpha$ clumps at kpc-scales & 0 & $\mathrm{M}$ \\
$20100-4156$ & non-ordered motions & 2 nuclei, tail & 1 & $\mathrm{I}$ \\
F22491-1808 & large-scale streaming motions & 2 nuclei, tails, diffuse H $\alpha$ & 0 & $\mathrm{I}$ \\
\hline
\end{tabular}

Notes.

Column (1): Target name.

Column (2): Simplified classification for the stellar motions from the pPXF analysis.

Column (3): Main morphological features.

Column (4): Number of companions in the MUSE FOV; see Table A.1 for details.

Column (5): IC classification.

regions, separated by $\sim 1.5 \mathrm{kpc}$ and with a stellar velocity offset of $\sim 60 \mathrm{~km} / \mathrm{s}$. In contrast, its nucleus, located $\sim 0.5^{\prime \prime}$ south of the southern SB based on ALMA data (Pereira-Santaella et al., in prep.), is highly obscured and not associated with strong optical emission. F19297-0406 was originally classified as a merger (e.g. Kim et al.2013); the inspection of near-IR HST (Fig. C.67) and ALMA (Pereira-Santaella et al., in prep.) allowed the identification of two nuclei, separated by $\sim 1.1 \mathrm{kpc}$. This source is therefore classified here as interacting (i.e. IIIb). For all remaining sources in our MUSE sample, we instead confirm the morphological classification reported in the literature.

The separation in the four sub-samples highlights that merging systems are more likely associated with ordered disk-like motions (8 out of 11, i.e. F00188-0856, IZw1, F01572+0009, F05189-2524, 13120-5453, F14378-3651, 17208-0014, and $19542+1110)$, while interacting systems are generally associated with non-ordered or streaming motions ( 7 out of 10 , i.e. F11095-0238, F12072-0444, F13451+1232, F14348-1447, F19297-0406, 20100-4156, and F22491-1808; see also e.g. Bellocchi et al. 2016 for similar results). The morphological class (i.e. interacting or merger) therefore is consistent in general with the stellar kinematic classification (i.e. dominant non-ordered or ordered motions); we note, however, that even when rotation patterns are observed, tidal streams are also present, indicating that dynamical relaxation times depend on galactocentric distances: Kinematic disturbances from interactions are indeed expected to fade within a few rotation cycles (e.g. Dale et al. 2001, Kronberger et al. 2007), and after the coalescence phase, the most external structures therefore require longer times to follow the rotation pattern and preserves irregular tidally induced velocities.

Indications of ordered motions in merger remnants have been reported in the literature: for instance, Barrera-Ballersteros et al.
(2015) traced the stellar kinematics in CALIFA merging galaxies, and found that $\sim 90 \%$ of merger remnants exhibit disk-like motions (see their Figs. B.3 and B.4); moreover, K-band observations reported by Rothberg \& Joseph (2004) revealed that most of the mergers in their sample show disky isophotals. Numerical simulations suggest that the number of stellar disks that survives or re-forms after an interaction is a strong function of the stellar mass ratio and gas content of the interacting galaxies: For instance, gas-rich mergers can yield disk-dominated remnants, while for modest gas fractions, the remnants are likely to resemble spheroidal-like galaxies (e.g. Hopkins et al. 2009a b; Naab \& Ostriker 2017).

We finally note that the $\mathrm{H} \alpha$ flux and stellar velocity maps in the simplified $\alpha_{A G N}-$ IC diagram (Fig 4) do not show clear trends, but are overall consistent with the Sanders et al. (1988) evolutionary scenario. In particular, more compact and dynamically relaxed systems are more likely to be found in the mergerAGN class, while interacting galaxies more likely present extended structures with several additional nuclear clumps and knots of SF.

\section{ISM nuclear properties: Outflows and ionisation conditions}

In this section, we report the general ISM properties of the ULIRG nuclear regions obtained from analysing MUSE data. In particular, we revise the optical classification of the nuclear spectra and infer the possible presence of atomic outflows, taking advantage of the unprecedented quality of MUSE data. This information is required to understand whether the PUMA sample, selected to characterise multi-phase outflows, does host gas flows that might be due to SB and AGN winds. 

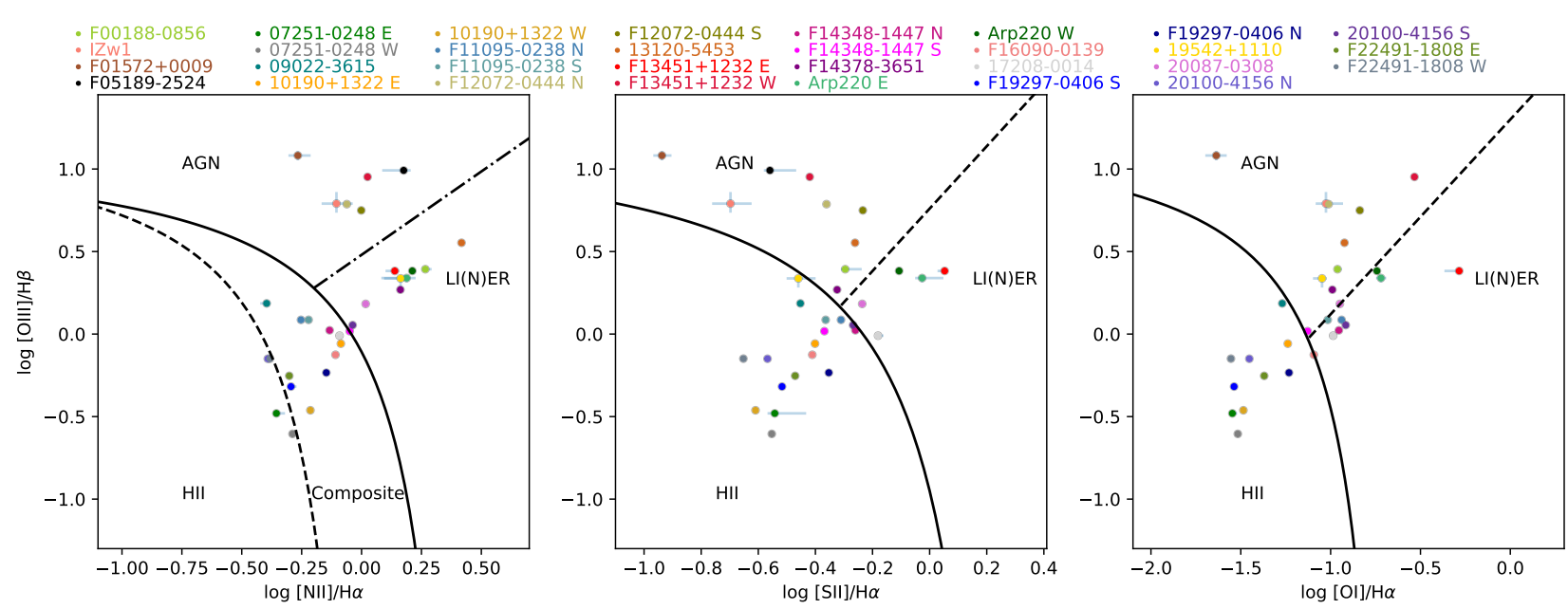

Fig. 6. Standard diagnostic BPT diagrams showing our flux ratio measurements obtained from the ULIRG nuclear spectra. Each nucleus is identified with a distinct colour, as labelled. Black curves separate HII-, composite-, AGN- and LI(N)ER-like line ratios, as labelled in the three panels (see text for details).

Thirty-one nuclear spectra were extracted from circular apertures centred at the position of the ULIRG nuclei, with $r<0.4^{\prime \prime}$ (i.e. considering our average spatial resolution). As a first step, we used pPXF to model the stellar contribution (Sect. 5), and after subtracting it, we used our own suite of python scripts to simultaneously fit all ISM prominent features. In particular, we modelled all emission lines with a combination of Gaussian profiles to account for both narrow and BLR features in type $1 \mathrm{AGN}$, or more in general, for asymmetric profiles with broad wings, that may trace perturbed kinematics and outflows. Na ID absorption is instead fit with a model parameterised in the optical depth space, as has been described in Perna et al. (2020). In the next section, we describe the modelling prescription for the ISM features of all but IZw1 and F01572 nuclei, the two Sy 1 whose prescription is presented in Appendix B

\subsection{ISM feature modelling}

We modelled the $\mathrm{H} \beta$ and $\mathrm{H} \alpha$ lines, the $\mathrm{HeI} \lambda 5876$, the [O III] $\lambda \lambda 44959,5007$, [N II] $\lambda \lambda 6548,83$, [S II] $\lambda \lambda 6716,31$, and [O I] $\lambda \lambda 6300,64$ doublets with Gaussian profiles. We constrained the wavelength separation between emission lines in accordance with atomic physics; moreover, we fixed the FWHM to be the same for all the emission lines. Finally, the relative flux of the two [N II] and [O III] components was fixed to 2.99 , the relative flux of the two [O I] lines was fixed to 3.13 , and the [S II] flux ratio was required to be within the range $0.44<$ $f(\lambda 6716) / f(\lambda 6731)<1.42$ (Osterbrock \& Ferland 2006).

To account for potential asymmetric line profiles, we performed each spectral fit four times at maximum, with one to four kinematic components (i.e. Gaussian sets, each centred at a given velocity and with a given FWHM). The final number of kinematic components used to model the spectra was derived on the basis of the Bayesian information criterion (BIC, Schwarz 1978; see e.g. Perna et al. 2019).

We simultaneously modelled the contribution of Na ID resonant lines. When sodium emission was detected, Gaussian profiles were used, requiring a Na ID doublet line ratio between the optically thick $(f(H) / f(K)=1)$ and thin $(f(H) / f(K)=2)$ limits (e.g. Rupke \& Veilleux 2015), where $H$ and $K$ indicate the sodium transitions at 5891 and $5896 \AA$, respectively. The $\mathrm{Na}$ ID absorption contribution was instead fitted with a model parametrised in the optical depth space, following Sato et al. (2009),

$$
\begin{aligned}
I(\lambda) & =I_{e m}(\lambda) \times f_{A B S}(\lambda) \\
& =I_{e m}(\lambda) \times\left(1-C_{f} \times\left[1-\exp \left(-\tau_{0} e^{-\left(\lambda-\lambda_{K}\right)^{2} /\left(\lambda_{K} b / c\right)^{2}}-\right.\right.\right. \\
& \left.\left.\left.2 \tau_{0} e^{-\left(\lambda-\lambda_{H}\right)^{2} /\left(\lambda_{H} b / c\right)^{2}}\right)\right]\right),
\end{aligned}
$$

where $H$ and $K$ indicate the two sodium transitions, $C_{f}$ is the covering factor, $\tau_{0}$ is the optical depth at the line centre $\lambda_{K}$, $b$ is the Doppler parameter $(b=F W H M /[2 \sqrt{\ln (2)}])$, and $c$ is the light velocity. The term $I_{e m}(\lambda)$ in Eq. 1 represents the intrinsic (unabsorbed) intensity, defined as $I_{*}+I_{H e I}$, where $I_{*}$ is the best-fit model obtained from the pPXF analysis, and $I_{\mathrm{HeI}}$ is the helium line intensity (Baron et al. 2020). When more than one kinematic component was required to fit Na ID absorption features, we assumed the case of partially overlapping atoms on the line of sight, so that the total sodium profile can be reproduced by multiple components and $I(\lambda)=I_{e m}(\lambda) \times \prod_{i=1}^{n} f_{A B S}^{i}(\lambda)$, where $f_{A B S}^{i}(\lambda)$ is the $i$ th component (as given in Eq. 1 ) used to model the sodium features (see also Sect. 3.1 in Rupke et al. 2002). We stress here that the kinematics of a given Na ID component (either in absorption or emission) are tied to those of a corresponding Gaussian set used to model the ISM emission lines.

Best-fit models for individual nuclear regions in our ULIRGs sample are reported in Appendix C. These fit results are used to investigate the dominant ionisation mechanism(s) for the emitting gas, and to derive the incidence of neutral and ionised outflows.

\subsection{BPT diagnostics}

Figure 6 shows the BPT diagrams with the results from our spectroscopic analysis. All line ratios were derived by integrating the line flux over the entire fitted profiles (i.e. considering all kinematic components used to model the lines). For simplicity, hereinafter the [O III] $\lambda 5007 / \mathrm{H} \beta$ versus [N II] $\lambda 6583 / \mathrm{H} \alpha$ (left panel), the $[\mathrm{O}$ III $] \lambda 5007 / \mathrm{H} \beta$ versus $[\mathrm{S}$ II $] \lambda \lambda 6716,31 / \mathrm{H} \alpha$ (centre), and [O III] $\lambda 5007 / \mathrm{H} \beta$ versus [O II] $\lambda 6300 / \mathrm{H} \alpha$ (right) are labelled [N II]-, [S II]-, and [O I]-BPT diagrams, respectively. In the figure, each coloured dot identifies a unique nuclear spectrum, as labelled at the top. 

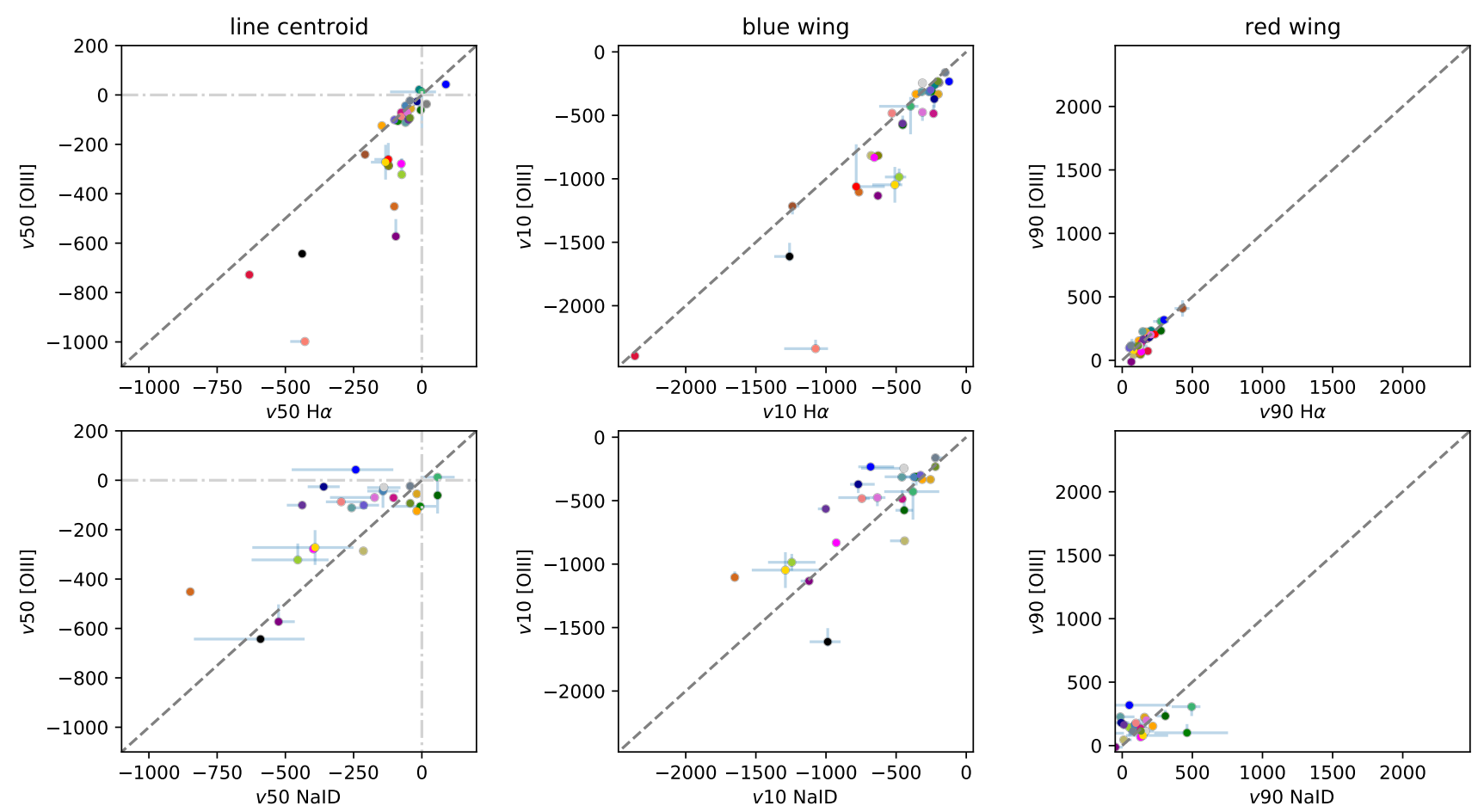

Fig. 7. Velocity-velocity diagram used to compare the non-parametric velocities of [O III], $\mathrm{H} \alpha$, and $\mathrm{Na}$ ID gas for the 31 nuclei reported in Table 4 Each nucleus is identified with a distinct colour (see labels in Fig. 6). In the left column, we show the central velocities of [O III] vs. H $\alpha$ (top) and of [O III] vs. Na ID (bottom); horizontal and vertical dot-dashed lines mark the zero-velocity positions, and the dashed line displays the 1:1 relation. In the central column, we show the $v 10$ velocities, associated with the blue wings of [O III] and $\mathrm{H} \alpha$ (top), and of [O III] and Na ID (bottom); the dashed line indicates the 1:1 relation. In the right column, we show the $v 90$ velocities, associated with the red wings of [O III] and $\mathrm{H} \alpha$ (top) and of [O III] and $\mathrm{Na}$ ID (bottom); the dashed line indicates the 1:1 relation.

The curves drawn in the [N II]-BPT diagram correspond to the theoretical boundary for extreme SBs (Kewley et al. 2001) and the empirical relation (Kauffman et al. 2003) used to separate purely $\mathrm{SF}$ galaxies from composite AGN-SF galaxies and AGN-/LI(N)ER-dominated systems (e.g. Kewley et al. 2006; Belfiore et al. 2016); the dot-dashed line is from Cid Fernandes et al. (2010), and is used to separate LI(N)ERs and AGN. The curves in the [S II]- and [O I]-BPT diagrams correspond to the optical classification scheme of Kewley et al. (2006, 2013), and are also used in this case to separate SF galaxies from AGN and LI(N)ER systems, as labelled in the figures.

For the sources for which at least two BPT diagnostics indicate the same region, we constrained the dominant ionisation mechanism responsible for the line emission: 11 out of 31 nuclei are associated with SF, 9 out of 31 with AGN, and 6 out of 31 with $\mathrm{LI}(\mathrm{N})$ ER ionisation. For the remaining five spectra, the BPT diagnostics provide inconsistent results, i.e. the three diagnostics indicate three different ionisation mechanisms.

Our flux ratio measurements are distributed over all the different regions of the BPT diagrams, and the inferred classification is overall consistent with that reported in the literature (Table 11, although the classifications in the literature were obtained from long-slit (spatially integrated) spectra. Our optical classification is also generally consistent with the $\alpha_{A G N}$ values reported in Table 1 The only exception in this case might be represented by 09022-3615, for which we observe SF-like line ratios, although the $\alpha_{A G N}=0.54$ was derived with mid-IR diagnostics; our results are instead consistent with the small (near-IR based) AGN fraction inferred by Nardini et al. (2010, see Table 11. These findings could suggest an extremely obscured AGN in this system (see also Sect. 5.3). We also note that 11 out of 17 nuclei with $\alpha_{A G N}<0.32$ have LI(N)ER-like ratios, possibly indicating shocks induced by SB-driven outflows or gravitational interactions. In fact, while in many local galaxies, the LI(N)ER emission is associated with gas ionised by the hard radiation field of evolved (post-AGB) stars and with $\mathrm{H} \alpha$ equivalent widths $<3$ (e.g. Belfiore et al.2016), this does not apply to our ULIRG nuclear regions.

\subsection{Multi-phase outflow incidence}

Almost all nuclear spectra show asymmetric and broad line profiles, possibly indicating strongly perturbed gas kinematics in both neutral (Na ID) and ionised (e.g. [O III], $\mathrm{H} \alpha$ ) atomic components. To characterise the overall kinematic properties of the ISM gas in a homogeneous way (i.e. to avoid any dependence on the number of distinct kinematic components used to model the line features in individual spectra), we used the non-parametric velocities $v 10, v 50$, and $v 90$, defined as the 10th, 50th, and 90th percentile velocities of the fitted line profiles, respectively, and the line width $w 80$, that is, the difference between the 85th and 15th percentile velocities (see e.g. Harrison et al. 2014). All velocities are derived with respect to the systemic velocity, as inferred from the stellar velocities measured from pPXF (see Sec. 57. [O III] and $\mathrm{H} \alpha$ line features are used as tracers for the ionised gas kinematics; instead, ISM neutral atomic gas kinematics are traced by the Na ID absorption component, detected in almost all nuclear spectra and showing the broadest profiles compared with the Na ID emission component (detected in only a few targets).

Figure 7 (first column) shows the velocity-velocity diagrams (VVD) that compare the central velocities (v50) of [O III] and $\mathrm{H} \alpha$ in the top panel and the VVDs of [O III] and Na ID in the 

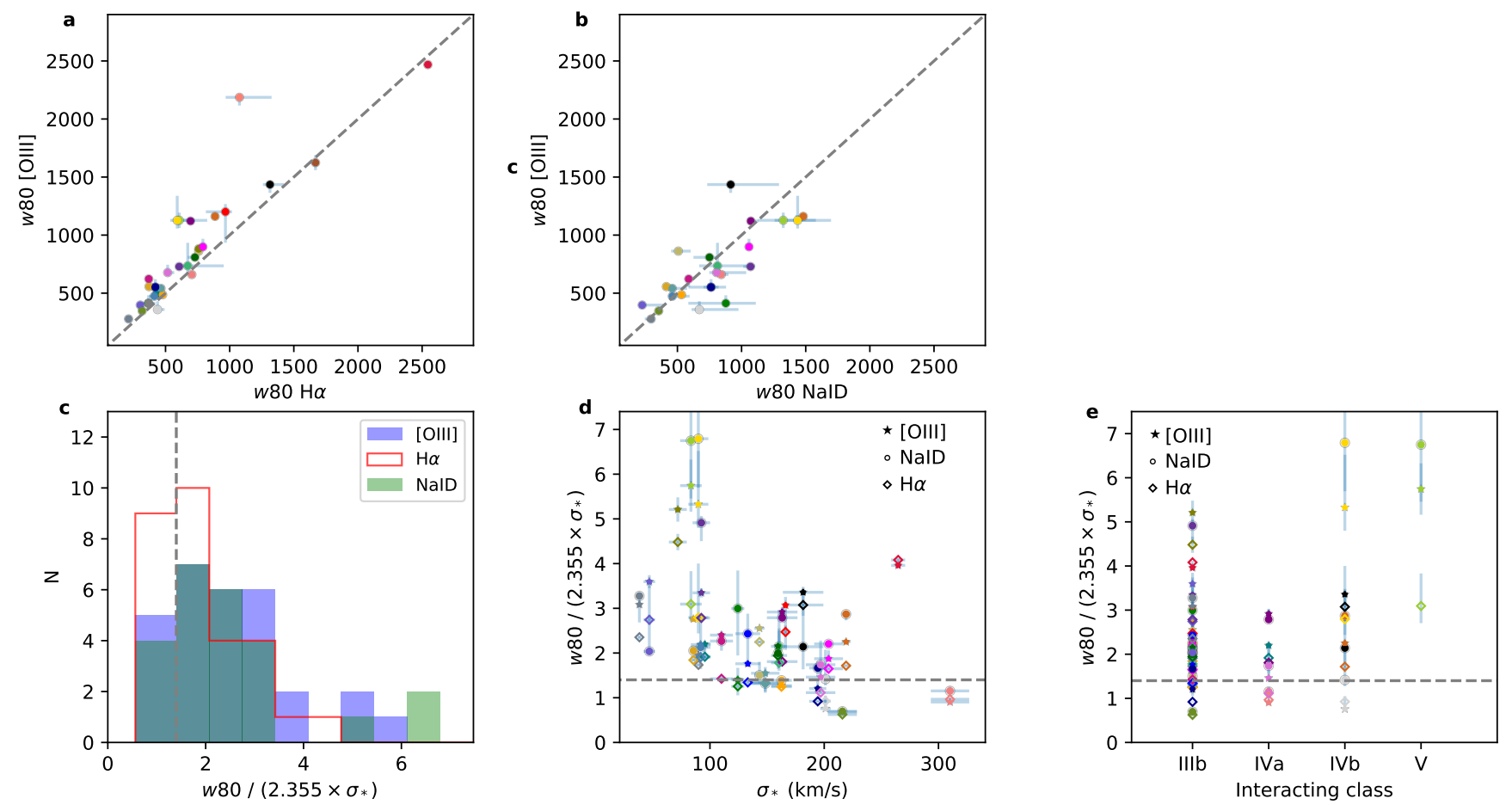

Fig. 8. Panels $a$ and $b$ : $w 80$ velocity-velocity diagram for [O III] vs. $\mathrm{H} \alpha(a)$, and [O III] vs. Na ID (b). Each nucleus is identified with a distinct colour (see labels in Fig. 6). Dashed lines display the 1:1 relation. Panel $c$ : Distributions of $\eta=w 80 /\left(2.355 \times \sigma_{*}\right)$ for the three kinematic tracers, as labelled in the legend; the dashed vertical line marks the position $\eta=1.4$ (see text). Panel $d$ : [O III] (stars), H $\alpha$ (diamonds), and Na ID (circles) $\eta$ measurements as a function of $\sigma_{*}$. None of the velocity dispersions and $w 80$ measurements are corrected for instrumental broadening; this correction would affect the only two $\sigma_{*}$ measurements close to the MUSE spectral resolution $(\sim 50 \mathrm{~km} / \mathrm{s})$, associated with the $20100-4156 \mathrm{~N}$ and F22491-1808 W nuclei, further increasing their $\eta$ values. Panel e: [O III] (stars), $\mathrm{H} \alpha$ (diamonds), and Na ID (circles) $\eta$ measurements as a function of the IC. The dashed horizontal line in panels $\mathrm{d}$ and e marks the position $\eta=1.4$.

bottom panel. Almost all line centroids are blueshifted with respect to the systemic velocity, indicating a significant contribution from approaching emitting ([O III] and $\mathrm{H} \alpha$ ) and absorbing (Na ID) material. This is confirmed by the fact that generally, the line profiles show very prominent blue wings, and faint and less extended red wings, as also shown in the central and right columns of Fig. 7, presenting $v 10$ and $v 90$ VVD, respectively.

In general, [O III] shows more extreme velocities than those of $\mathrm{H} \alpha$ (see also e.g. Bae \& Woo al. 2014, Venturi et al. 2018, and Cicone et al.2016 for similar results; but see also Rodríguez del Pino et al. 2019). The Na ID velocities in turn appear slightly higher than those of the [O III], although the former are associated with higher uncertainties: $\langle v 10$ [O III] $/ v 10 \mathrm{H} \alpha\rangle=1.27$ and $\langle v 10 \mathrm{Na}$ ID $/ v 10[\mathrm{O}$ III] $\rangle=1.16$. The high uncertainties in Na ID velocities are due to the fit degeneracy. In particular, they could originate from a blending between the HeI (in emission) and the Na ID features when strong sodium absorption is detected at very high negative velocities (e.g. F05189-2524 and F14378-3651). Alternatively, it can originate from the modelling of Na ID P Cygni profiles (F05189-2524 and F11095-0238 NE).

In Fig. 8 (panels $a$ and $b$ ) we show the $w 80$ VVD. In this case, we also observe that in general, Na ID $w 80$ are slightly higher than $[\mathrm{O}$ III] $w 80$, which in turn are higher than $\mathrm{H} \alpha$ line widths: $\langle w 80$ [O III] $/ w 80 \mathrm{H} \alpha\rangle=1.15$ and $\langle w 80 \mathrm{Na}$ ID $/ w 80$ $[\mathrm{O}$ III $]\rangle=1.10$. In order to distinguish between gravitational and outflow processes that might cause the velocity shifts and the extreme $v 10$ and $w 80$ in the [O III], $\mathrm{H} \alpha$ and $\mathrm{Na}$ ID lines, we compared their line widths with the stellar velocity dispersion at the position of the nuclei. Following Woo et al. (2016), we assumed that a non-gravitational component dominates the gravitational component when $\eta=w 80 /\left(2.355 \times \sigma_{*}\right)>1.4$, where the stellar velocity dispersion $\sigma_{*}$ traces the gravitational motions. This is a conservative criterion, as originally proposed for type $2 \mathrm{AGN}$ and applied here to ULIRG nuclear spectra: In fact, $\sigma_{*}$ may be enhanced by motions associated with the merging process in ULIRGs.

In panel $\mathrm{c}$ of Fig. 8 we report the histograms of the $\eta$ measurements, as obtained for the three kinematic tracers; in panel $d$, we instead show $\sigma_{*}$ as a function of $\eta$, for $\mathrm{H} \alpha$, [O III], and $\mathrm{Na}$ ID features. We derived $\eta<1.4$ from both [O III] and Na ID for only three nuclei: 10190+1322 E, F16090-0139, and F22491-1808 E. The $\mathrm{H} \alpha$ distribution instead shows nine nuclei with $\eta<1.4$, again indicating that the Balmer line can be less strongly affected by non-gravitational motions. For each tracer and nucleus, we indicate in Table 4 whether gravitational or non-gravitational motions dominate according to the chosen criterion.

We note that the $\eta$ criterion can be applied for all nuclei for which $(i) \sigma_{*}$ can be derived from pPXF analysis, and (ii) the specific kinematic tracer for ionised and neutral gas is detected. [O III] and $\mathrm{H} \alpha$ lines are detected in all spectra, but no stellar features are present in the IZw1 and F01572+0009 nuclear spectra; therefore ionised gas $\eta$ measurements were derived for all but these two Sy 1 nuclei. In contrast, Na ID absorption is not detected in 7 out of 31 nuclear spectra: the two Sy 1 targets, IZw1, and F01572+0009 show strong continuum and very broad line emission from the HeI at the position of the two Na ID transitions; moreover, although Na ID emission is detected, the strong BLR component prevents a robust analysis of a possible neutral outflow. The F13451+1332 W spectrum does not show sodium absorption (Fig. C.49), although a faint $\left(C_{f}=0.11\right)$ and broad $(b=400 \mathrm{~km} / \mathrm{s}$ ) Na ID profile in absorption has been reported in Rupke et al. (2005c). The Na ID emission component that we 

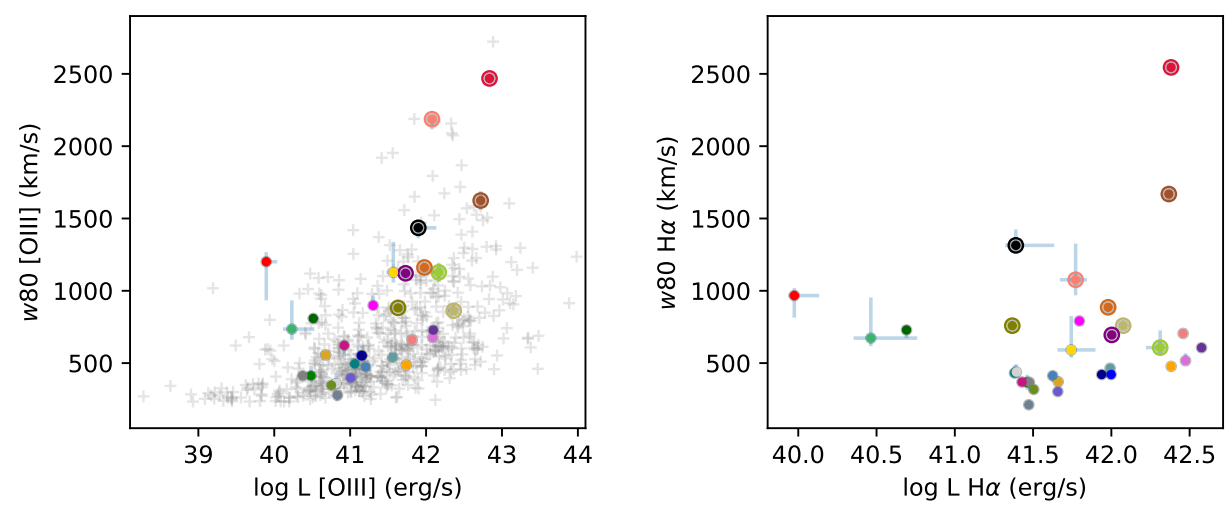

Fig. 9. Left: [O III] non-parametric velocity $w 80$ as a function of $\mathrm{L}_{[O I I I]}$ for the ULIRG nuclei presented in this work (colour-coded as in Fig. 6), and the X-ray/SDSS AGN (grey crosses) from Perna et al. (2017). Larger symbols identify the ULIRG nuclei with AGN ionisation (see Table 4). Right: $\mathrm{H} \alpha$ non-parametric velocity $w 80$ as a function of $\mathrm{L}_{H \alpha}$ for the ULIRG nuclei presented in this work.
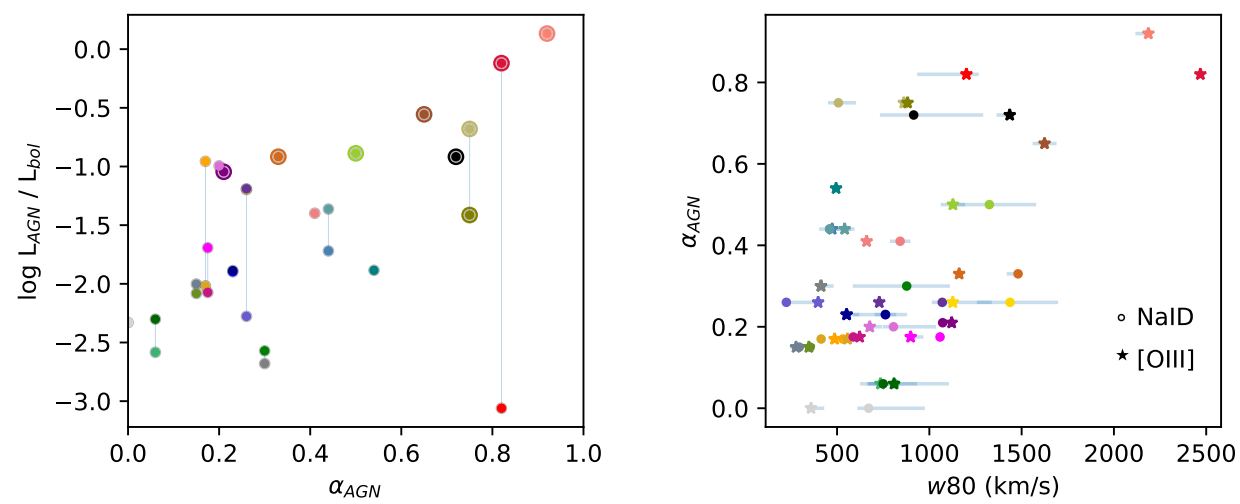

Fig. 10. Left: (mid-IR based) AGN fraction $\alpha_{A G N}$ as a function of the ratio $\mathrm{L}_{A G N} / \mathrm{L}_{b o l}$, with $\mathrm{L}_{A G N}=3 \times 10^{3} \mathrm{~L}_{[O I I I]}$ and $\mathrm{L}_{b o l}=1.15 \times \mathrm{L}_{I R}$. The nuclei of binary systems are connected with a vertical line; larger symbols refer to the sources with dominant AGN ionisation according to our BPT diagnostics. Right: [O III] and Na ID non-parametric velocity $w 80$ as a function of the AGN fraction $\alpha_{A G N}$, defined on the basis of mid-IR diagnostics (Sect. 11). Each nucleus is identified with a distinct colour, as in Fig.6

used to model the observed spectrum might not be as reliable because pPXF best-fit results are not robust (no strong stellar absorbing features are detected over the entire wavelength range covered with MUSE). Finally, for the remaining four spectra (07251-0248 W, F12072-0444 S, F13451+1232 E, and 090223615), the stellar Na ID contribution is responsible for the total absorption according to our pPXF analysis results. As a consequence, we can infer the presence/absence of dominant nongravitational motions in absorbing neutral gas for 24 nuclei.

For the sources with $\eta<1.4$ from both [O III] and Na ID kinematics (i.e. 10190+1322 E, F16090-0139, and F22491-1808 E), we note that in at least one sources, F16090-0139, the stellar velocity dispersion might be overestimated (because of the low $\mathrm{S} / \mathrm{N}$ ), while the ISM features are extremely broad (e.g. Na ID $w 80 \sim 840 \mathrm{~km} / \mathrm{s}$; see Fig. C.62). This nucleus presents the highest $\sigma_{*}$ in our sample (see Fig. 8, panel $d$ ). Hence, the F160900139 nucleus is reasonably associated with an outflow. We also note that the two Sy 1 nuclei display extremely broad features, likely associated with outflows. Taking these arguments into account, we conclude that non-gravitational motions dominate the neutral (ionised) gas kinematics in 20 out of 24 (28 out of 31) nuclei.

Finally, panel e of Fig. 8 shows the $\eta$ measurements as a function of the IC of each target. No clear trend is observed for the three gas kinematic tracers (i.e. Na ID, $\mathrm{H} \alpha$, and [O III], with Spearman coefficients of $\sim 0.1$ ). Despite the poor statistics, this finding suggests that nuclear winds are ubiquitous during the pre- and post-coalescence phases of major mergers. The dominant non-gravitational component in almost all ULIRG nuclei, together with the prevalent prominent blue wings in [O III] and $\mathrm{Na}$ ID, with $v 10$ of several $100 \mathrm{~s} \mathrm{~km} / \mathrm{s}$, indicates strong multiphase outflows driven by either AGN or SB nuclear activity.

\subsection{AGN- and SB-driven outflows}

In Sect. 5.3 we reported a good consistency between the morphological and stellar kinematic classifications, with merging systems more likely associated with ordered disk-like motions (in 8 out of 11 of our ULIRGs) and interacting systems with non-ordered or streaming motions (in 7 out of 10 ULIRGs). The ubiquitous presence of atomic outflows (Sect. 6.3) suggests that nuclear winds are not related to a specific phase or to more/less dynamically relaxed systems, but are common in all final stages of the merger process (as has been reported in the literature; see e.g. Sect. 10.5 in Perna et al. 2020). In this section, we therefore investigate the possible connection between AGN and SB activity and the atomic outflow velocities.

Figure 9 (left) shows the distribution of the [O III] $w 80$ measurements against the [O III] luminosity. In the same figure (right), we also report the $\mathrm{H} \alpha w 80$ versus $\mathrm{L}_{H \alpha}$. The [O III] and $\mathrm{H} \alpha$ luminosities were corrected for extinction, considering the measured Balmer decrement and a Cardelli et al. (1989) extinction law. The [O III] measurements show a positive trend with increasing luminosity (with a Spearman coefficient of 0.6), while 
Table 4. ISM nuclear properties

\begin{tabular}{|c|c|c|c|c|c|c|}
\hline \multirow{2}{*}{$\begin{array}{l}\text { IRAS ID (other) } \\
\text { (1) }\end{array}$} & \multirow{2}{*}{$\begin{array}{l}\text { optical } \\
\text { class } \\
\text { (2) }\end{array}$} & \multirow{2}{*}{$\begin{array}{l}\text { BPTs } \\
\text { (3) }\end{array}$} & \multicolumn{3}{|c|}{$\begin{array}{l}\text { dominant motions } \\
\text { from } \eta \text { criterion }\end{array}$} & \multirow{2}{*}{$\begin{array}{c}\max w 80 \\
(\mathrm{~km} / \mathrm{s}) \\
(7)\end{array}$} \\
\hline & & & $\begin{array}{r}\mathrm{H} \alpha \\
(4)\end{array}$ & $\begin{array}{c}{[\mathrm{O}} \\
(5)\end{array}$ & $\begin{array}{c}\mathrm{Na} \text { ID } \\
(6)\end{array}$ & \\
\hline F00188-0856 & AGN & $(\mathrm{L}, \mathrm{A}, \mathrm{A})$ & non-grav & non-grav & non-grav & $1325_{-195}^{+255}(\mathrm{Na}$ ID) \\
\hline IZw1 & AGN & $(\mathrm{A}, \mathrm{A}, \mathrm{A})$ & - & - & - & $2190_{-70}^{+10}([\mathrm{O}$ III $])$ \\
\hline F01572+0009 & AGN & $(\mathrm{A}, \mathrm{A}, \mathrm{A})$ & - & - & - & $1670_{-60}^{+10}([\mathrm{O} \mathrm{III}])$ \\
\hline F05189-2524 & AGN & $(\mathrm{A}, \mathrm{A}, \mathrm{A})$ & non-grav & non-grav & non-grav & $1860_{-10}^{+130}([\mathrm{O}$ III $])$ \\
\hline $07251-0248 \mathrm{E}$ & SB & $(\mathrm{S}, \mathrm{S}, \mathrm{S})$ & grav & non-grav & non-grav & $880_{-200}^{+230}(\mathrm{Na}$ ID) \\
\hline $07251-0248 \mathrm{~W}$ & SB & $(\mathrm{S}, \mathrm{S}, \mathrm{S})$ & non-grav & non-grav & - & $410 \pm 5([\mathrm{O}$ III $])$ \\
\hline $09022-3615$ & SB & $(\mathrm{C}, \mathrm{S}, \mathrm{S})$ & non-grav & non-grav & - & $495 \pm 5([\mathrm{O}$ III $])$ \\
\hline $10190+1322 \mathrm{E}$ & SB & $(\mathrm{C}, \mathrm{S}, \mathrm{S})$ & grav & grav & grav & $530_{-20}^{+5}(\mathrm{Na}$ ID) \\
\hline $10190+1322 \mathrm{~W}$ & SB & $(\mathrm{C}, \mathrm{S}, \mathrm{S})$ & non-grav & non-grav & non-grav & $555_{-5}^{+5}([\mathrm{O} \mathrm{III}])$ \\
\hline F11095-0238 NE & $?$ & $(\mathrm{C}, \mathrm{S}, \mathrm{L})$ & non-grav & non-grav & non-grav & $610_{-70}^{+10}([\mathrm{O}$ III $])$ \\
\hline F11095-0238 SW & $?$ & (C,S,L) & grav & non-grav & grav & $540 \pm 5($ [O III] $)$ \\
\hline F12072-0444 N & AGN & $(\mathrm{A}, \mathrm{A}, \mathrm{A})$ & non-grav & non-grav & non-grav & $860 \pm 5([\mathrm{O}$ III] $)$ \\
\hline F12072-0444 S & AGN & $(\mathrm{A}, \mathrm{A}, \mathrm{A})$ & non-grav & non-grav & - & $880 \pm 5([\mathrm{O}$ III $])$ \\
\hline $13120-5453$ & AGN & $(\mathrm{L}, \mathrm{A}, \mathrm{A})$ & non-grav & non-grav & non-grav & $1480_{-60}^{+5}(\mathrm{Na}$ ID) \\
\hline $\mathrm{F} 13451+1232 \mathrm{E}$ & LI(N)ER & $(\mathrm{L}, \mathrm{L}, \mathrm{L})$ & non-grav & non-grav & - & $1200_{-270}^{+60}([\mathrm{O}$ III $])$ \\
\hline $\mathrm{F} 13451+1232 \mathrm{~W}(4 \mathrm{C} 12.50)$ & AGN & $(\mathrm{A}, \mathrm{A}, \mathrm{A})$ & non-grav & non-grav & - & $2545 \pm 5(\mathrm{H} \alpha)$ \\
\hline F14348-1447 NE & $?$ & $(\mathrm{C}, \mathrm{S}, \mathrm{L})$ & non-grav & non-grav & non-grav & $620_{-70}^{+10}([\mathrm{O}$ III $])$ \\
\hline F14348-1447 SW & $?$ & $(\mathrm{~L}, \mathrm{~S}, \mathrm{~A})$ & non-grav & non-grav & non-grav & $1060 \pm 5(\mathrm{Na}$ ID $)$ \\
\hline F14378-3651 & AGN & $(\mathrm{L}, \mathrm{A}, \mathrm{A})$ & non-grav & non-grav & non-grav & $1120 \pm 5([\mathrm{O} \mathrm{III}])$ \\
\hline Arp220 E & LI(N)ER & $(\mathrm{L}, \mathrm{L}, \mathrm{L})$ & non-grav & non-grav & non-grav & $810_{-140}^{+300}(\mathrm{Na}$ ID) \\
\hline Arp220 W & LI(N)ER & $(\mathrm{L}, \mathrm{L}, \mathrm{L})$ & non-grav & non-grav & non-grav & $810 \pm 5([\mathrm{O}$ III $])$ \\
\hline F16090-0139 & SB & $(\mathrm{C}, \mathrm{S}, \mathrm{S})$ & grav* $^{*}$ & grav* $^{*}$ & grav* $^{*}$ & $840_{-55}^{+60}(\mathrm{Na}$ ID) \\
\hline $17208-0014$ & LI(N)ER & $(\mathrm{C}, \mathrm{L}, \mathrm{L})$ & grav & grav & non-grav & $435_{-5}^{+55}(\mathrm{Na}$ ID) \\
\hline F19297-0406 S & SB & $(\mathrm{C}, \mathrm{S}, \mathrm{S})$ & grav & non-grav & non-grav & $760_{-180}^{+110}(\mathrm{Na}$ ID) \\
\hline F19297-0406 N & SB & $(\mathrm{C}, \mathrm{S}, \mathrm{S})$ & grav & grav & non-grav & $630_{-55}^{+10}(\mathrm{Na}$ ID) \\
\hline $19542+1110$ & $?$ & $(\mathrm{~L}, \mathrm{~S}, \mathrm{~A})$ & non-grav & non-grav & non-grav & $1440_{-180}^{+260}(\mathrm{Na}$ ID) \\
\hline $20087-0308$ & LI(N)ER & $(\mathrm{L}, \mathrm{L}, \mathrm{L})$ & grav & non-grav & non-grav & $800_{-50}^{+230}(\mathrm{Na}$ ID) \\
\hline $20100-4156 \mathrm{~N}$ & SB & $(\mathrm{S}, \mathrm{S}, \mathrm{S})$ & non-grav & non-grav & non-grav & $400 \pm 5([\mathrm{O}$ III $])$ \\
\hline $20100-4156 \mathrm{~S}$ & LI(N)ER & $(\mathrm{L}, \mathrm{S}, \mathrm{L})$ & non-grav & non-grav & non-grav & $560_{-380}^{+400}(\mathrm{Na}$ ID) \\
\hline F22491-1808 E & SB & $(\mathrm{C}, \mathrm{S}, \mathrm{S})$ & grav & grav & grav & $355 \pm 5$ (Na ID) \\
\hline F22491-1808 W & SB & $(\mathrm{S}, \mathrm{S}, \mathrm{S})$ & non-grav & non-grav & non-grav & $295_{-50}^{+10}(\mathrm{Na}$ ID) \\
\hline
\end{tabular}

Notes.

Column (1): Target name.

Column (2): Optical classification obtained from the three BPT diagrams shown in Fig. 6 The classification is defined only when two or three diagnostic diagrams indicate the same ionisation mechanism; when the three diagnostics indicate three different mechanisms, a question mark is placed in this column.

Column (3): Ionisation class from the [N II]-, [S II]-, and [O I]-BPT diagostics (S: HII, C: composite, A: AGN, and L: LI(N)ER line ratios).

Columns (4-6): Dominant motions from $\eta=w 80 /\left(2.355 \times \sigma_{*}\right)$, considering the $\mathrm{H} \alpha$, [O III], and Na ID line widths; see Sect. 6.4 for details.

Column (7): Maximum w80 measured in the $\mathrm{H} \alpha$ and [O III] emission lines as well as the Na ID absorption features.

${ }^{*}$ The asterisk indicates that the stellar velocity dispersion in the F16090-0139 nucleus is probably overestimated $\left(\sigma_{*}=310 \mathrm{~km} / \mathrm{s}\right)$ because of the low $\mathrm{S} / \mathrm{N}$.

$\mathrm{H} \alpha$ does not show any significant correlation; we also note that our ULIRGs cover similar region in the [O III] diagram as the X-ray/SDSS AGNs analysed in Perna et al. (2017), reported in our figure with grey pluses. This might suggest that (i) the AGN activity, traced by $\mathrm{L}_{[O I I I]}$, is responsible for the outflows in the ULIRG nuclei, or that (ii) AGN- and SB-driven winds are hardly distinguishable in this diagram.

To test these two scenarios, we studied the correlation between two different estimates for the AGN fraction: (i) $\alpha_{A G N}$, the AGN contribution to the $\mathrm{L}_{b o l}$ according to mid-IR fluxes (Sect. 11), and (ii) $L_{A G N} / L_{b o l}$, the ratio between the [O III]-based AGN luminosity (inferred assuming a bolometric correction of $3 \times 10^{3}$; Heckman et al. 2004), and the bolometric luminosity $\mathrm{L}_{\text {bol }}$, given by $1.15 \times L_{I R}$ (Veilleux et al. 2009). A positive correlation between these quantities is expected if [O III] traces the AGN power. In Fig. 10 (left), we report $\alpha_{A G N}$ as a function of $L_{A G N} / L_{b o l}$. A significant correlation (with a Spearman coeffi- cient of 0.6) is found when we exclude the most deviating measurement, namely the red dot associated with F13451+1232 E (whose AGN fraction is reasonably overestimated; see below). Therefore this finding is in principle consistent with the hypothesis that the AGN activity causes the outflows we observe in almost all ULIRG nuclei.

However, there are a number of caveats concerning the relation in the left panel of Fig. 10. Both $\mathrm{L}_{b o l}$ and $\alpha_{A G N}$ are based on spatially integrated IR emission measurements and refer to the entire ULIRG systems. We can reasonably assume that most of this IR emission comes from the nuclei (e.g. Lutz et al. 1999, Pereira-Santaella et al., in prep.), and that all quantities in the figure are related to the same spatial regions, at least for the targets with a single nucleus. For the binary systems, we used the same $\mathrm{L}_{I R}$ and $\alpha_{A G N}$ for both nuclei (these systems are connected with vertical lines in the figure). This is a reasonable assumption because the [O III] luminosities associated with the two nu- 
clei of individual systems are comparable within a factor of a few. The only exception is represented by F13451+1232, with $\mathrm{L}_{[O I I I]}=6.8 \times 10^{42} \mathrm{erg} / \mathrm{s}(\mathrm{W})$ and $7.8 \times 10^{39} \mathrm{erg} / \mathrm{s}(\mathrm{E})$. The western nucleus, associated with AGN activity (Fig. 6), harbours the powerful radio source 4C 12.50 (Lister et al. 2003), and might cause most of the IR emission. If this is the case, the only deviating point in Fig. 10 (left), associated with F13451+1232 E, should have a much smaller $\alpha_{A G N}$. However, a detailed investigation is required to confirm all assumptions mentioned so far. Moreover, BPT diagnostics revealed that AGN dominates SB and LI(N)ER ionisation mechanisms in only nine nuclei (large symbols in Fig. 10, left); in the remaining nuclei, the [O III] luminosity is therefore a poor tracer for the AGN power. All these arguments weaken the significance of the trend reported in the left panel of Fig. 10 .

We also considered the correlation between the $\mathrm{Na}$ ID and [O III] line widths and $\alpha_{A G N}$ because broader line profiles are generally reported in the literature for AGN-driven outflows (e.g. Cazzoli et al. 2016, Kakkad et al. 2020). These measurements are reported in the right panel of Fig. 10 and do not show a clear trend (Spearman correlation of 0.4 ). We therefore conclude that although a significant contribution of [O III] might be due to AGN, a combination of SB- and AGN-driven winds might cause the observed atomic outflows. More detailed analyses of the outflow nature will be presented in future papers, however, together with detailed spatially resolved properties of neutral and ionised outflows (e.g. Perna et al. 2020).

We finally note that most of the nuclei with neutral outflows have relatively low AGN fractions; instead, at $\alpha_{A G N}>0.5, \mathrm{Na}$ ID outflows are present in only one nucleus, F05189-2524 (out of eight, see the right panel of Fig. 10, confirming the difficulties in observing neutral outflows in systems with strong AGN (e.g. Perna et al. 2017, Bae \& Woo 2018, Nedelchev et al. 2019). A detailed spatially resolved analysis is required, however, to exclude neutral outflows in the more external regions of these powerful AGN (e.g. Rupke et al.|2017; Perna et al., in prep.).

\section{Conclusions}

The project called Physics of ULIRGs with MUSE and ALMA (PUMA) is a survey of 25 nearby ULIRGs observed with MUSE and ALMA. This is a representative sample that covers the entire ULIRG luminosity range, and it includes a combination of systems with AGN and SB nuclear activity in (advanced) interacting and merging stages. This project represents the first such study intended to characterise the multi-phase structure of the ISM in local ULIRGs at subkpc resolutions. This paper is the first in a series that will explore the prevalence of ionised, neutral, and molecular outflows as a function of the galaxy and $\mathrm{BH}$ properties, and the nature and the (feedback) effects of such outflows on the galaxy evolution.

In this work, we presented the first data products obtained from analysing the MUSE data of the 21 ULIRGs observed so far. We described the stellar kinematics derived with the pPXF analysis, and the properties of stellar and ionised gas emission. Our first results are summarised below. Colour-composites, ionised gas, and stellar continuum emission images show a great richness in spatial details at different spatial scales. These images reveal recent galaxy interactions and strong (dust-enshrouded) nuclear activity, in line with their ULIRG nature (see e.g. Fig. 2).

Stellar kinematics revealed that merging systems are more likely associated with ordered disk-like motions (8 out of 11), while binary (interacting) systems are dominated by non-ordered and streaming motions ( 7 out of 10; see e.g. Fig. 57).

The sources with stellar rotational patterns are more likely found in compact mergers hosting AGN (6 out of 11); on the other hand, more extended structures with non-ordered motions are found in interacting systems, showing several additional nuclear SF clumps and strong tidal tails (Figs. 4 and 5).

All ULIRGs show distinct velocity structures that are detached from the the inner pattern through tidal tails. This also applies to the post-coalescence mergers with clear inner rotational patterns, and it confirms that the external structures require longer times to reach a dynamically relaxed configuration.

In the second part of the paper, we analysed the 31 nuclear spectra extracted from the positions of the ULIRG nuclei, deriving the physical and kinematic properties of the nuclear ISM. Our first results are summarised below. We used BPT diagnostics to constrain the dominant ionisation mechanism that causes the optical emission lines: 11 out of 31 nuclei are associated with SF, 9 out of 31 with AGN, and 6 out of 31 with LI(N)ER ionisation. Overall, the inferred classification is consistent with the classifications reported in the literature (Table 1), although the latter were obtained with long-slit spectra. Our classification is also consistent with the archival $\alpha_{A G N}$ estimates, obtained from mid-IR diagnostics. For the remaining 5 nuclei, the three BPT diagnostics indicated three different ionisation mechanisms, possibly indicating a more complex mixture between SB, AGN, and shock-induced ionisation (see Table 4).

Almost all nuclear spectra show asymmetric and broad line profiles in both neutral (Na ID) and ionised (e.g. [O III]) transitions. Nuclear ISM features display velocity dispersions $>0.15$ dex higher than $\sigma_{*}$ in $\sim 85 \%$ of the nuclear spectra. Following Woo et al. (2016), we considered these enhancements with respect to $\sigma_{*}$ as an indication of strong non-gravitational motions in the ISM component. Together with the extreme $v 10$ velocities associated with [O III] and Na ID lines (Fig. 7), this suggests the ubiquitous presence of powerful nuclear winds in our sample.

Most of the nuclei with neutral outflows have relatively low AGN fractions; at $\alpha_{A G N}>0.5$, Na ID outflows are instead present in one nucleus (out of 8, see Fig. 10, left), confirming the difficulties of observing neutral outflows in systems with strong AGN.

While in the this paper we have presented and described the general properties of the sample and the MUSE data, more detailed studies also involving ALMA data will be presented in future papers.

Acknowledgements. We thank the referee for an expert review of our paper. The authors thanks Elena Valenti for her support when preparing the observations, and G. Vietri for useful discussion on spectral analysis of type 1 AGN. MP is supported by the Programa Atracción de Talento de la Comunidad de Madrid via grant 2018-T2/TIC-11715. MP, SA, CTC and LC acknowledge support from the Spanish Ministerio de Economía y Competitividad through the grant ESP2017-83197-P, and PID2019-106280GB-I00. MPS acknowledges support from the Comunidad de Madrid through the Atracción de Talento Investigador Grant 2018-T1/TIC-11035 and PID2019-105423GAI00 (MCIU/AEI/FEDER,UE). EB acknowledges support from Comunidad de Madrid through the Attracción de Talento grant 2017-T1/TIC-5213. SC acknowledge financial support from the State Agency for Research of the Spanish MCIU through the "Center of Excellence Severo Ochoa" award to the Instituto de Astrofísica de Andalucía (SEV-2017-0709). ACG acknowledges support from the Spanish Ministerio de Economía y Competitividad through the grant BES2016-078214. RM acknowledges ERC Advanced Grant 695671 "QUENCH" and support by the Science and Technology Facilities Council (STFC). JPL acknowledges financial support by the Spanish MICINN under grant AYA201785170-R. 


\section{References}

Alcorn, L.Y., Tran K.-V., Glazebrook K., et al. 2018, ApJ, 858, 47 Arribas, S., Colina, L., Monreal-Ibero, A., et al. 2008, A\&A, 479, 687 Arribas, S., Colina, L., Alonso-Herrero, A., et al. 2012, A\&A, 541, 20 Arribas, S., Colina, L., Bellocchi, E., et al. 2014, A\&A, 568, 14A

Bacon, R., Accardo, M., Adjali, L., et al. 2010, in Society of Photo-Optical Instrumentation Engineers (SPIE) Conference Series, Vol. 7735, Society of Photo-Optical Instrumentation Engineers (SPIE) Conference Series, 773508 Bae, H. J. \& Woo, J. H. 2014, ApJ, 795, 30

Bae, H. \& Woo, J. 2018, ApJ, 853, 185

Baldwin, J. A., Phillips, M. M. \& Terlevich, R. 1981, PASP, 93, 5B

Baron, D., Netzer, N., Davies, R. I., Prochaska, J. X. 2020, MNRAS, 494, 5396

Barrera-Ballersteros, J. K., García-Lorenzo, Falcón-Barroso, J., et al. 2015, A\&A, 582, 21

Bedregal, A. G., Colina, L., Alonso-Herrero, A., Arribas S. 2009, ApJ, 698, 1852 Belfiore, F., Maiolino, R., Maraston, C., et al. 2016, MNRAS, 461, 3111

Belfiore, F., Westfall, K. B., Schaefer, A., et al. 2019, ApJ, 158, 160

Bellocchi, E., Arribas, S., Colina, L., Miralles-Caballero, D. 2013, A\&A, 557, 59

Bellocchi, E., Arribas, S., Colina, L. 2016, A\&A, 591, 85

Bischetti, M., Maiolino, R., Carniani, S., et al. 2019, A\&A, 630, 59

Bothwell, M. S., Smail, I., Chapman, S.C., et al. 2013, MNRAS, 429, 3047

Brusa, M., Bongiorno, A., Cresci, G., et al. 2015, MNRAS, 446, 2394

Cappellari, M., Copin, Y. 2003, MNRAS, 342, 345C

Cappellari, M. \& Emsellem, E. 2004, PASP, 116, 138C

Cappellari, M. 2017, MNRAS, 466, 798

Cardelli, J. A., Clayton, G. C., \& Mathis, J. S. 1989, ApJ, 345, 245

Carniani, S., Marconi, A., Maiolino, R., et al. 2016, A\&A, 591, 28

Casey, C. M., Narayanan, D., Cooray, A. 2014, PhR, 541, 45

Cazzoli, S., Arribas, S., Maiolino, R., Colina, L. 2016, A\&A, 590, A125

Ceverino, D., Klessen, R. S., Glover, S. C. O., et al. 2018, MNRAS, 480, 4842

Cicone, C., Maiolino, R., Sturm, E., et al. 2014, A\&A, 562, 21

Cicone, C., Maiolino, R., Marconi, A., 2016, A\&A, 588, 41

Cid Fernandes, R., Stasińska, G., Schlickmann, M. S. et al. 2010, MNRAS, 403 , 1036

Colina, L., Arribas, S., Borne, K.D. 1999, ApJ, 527, 13

Colina, L., Pereira-Santaella, M., Alonso-Herrero, A., et al. 2012, ApJ, 749, 116

Condon, J. J., Anderson, M. L., \& Helou, G. 1991, ApJ, 376, 95

Cresci, G., Mainieri, V., Brusa, M., et al. 2015, ApJ, 799, 81C

Davé, R., Anglés-Alcázar, D., Narayanan, D., et al. 2019, MNRAS, 486, 2827

Dale, D.A., Giovanelli, R., Haynes, M. P., et al. 2001, AJ, 121, 1886

Dekel, A., Birnboim, Y., Engel, G., 2009, Nature, 457, 451

Dekel, A., Sarkar, K. C., Fangzhou, J., et al., 2019, MNRAS, 488, 4753

Duc, P.-A., Mirabel, I. F., \& Maza, J. 1997, A\&AS, 124, 533

Emonts, B.H.C., Carilli, C., Narayanan, D., et al. 2018, ASPC, 517, 587

Feruglio, C., Maiolino, R., Piconcelli, E., et al. 2010, A\&A, 518, 155F

Fluetsch, A., Maiolino, R., Carniani, S., et al. 2019, MNRAS, 483, 4586

Fluetsch, A., Maiolino, R., Carniani, S., et al. 2020, arXiv:2006.13232

Föerster-Schreiber, N. M., Renzini, A., Mancini, C., et al. 2018, ApJS, 238, 21

Gadotti, D.A., Sánchez-Blázquez, P., Falcón-Barroso, J., et al. 2019, MNRAS, 482,506

Gaia Collaboration 2018, A\&A, 616, $1 \mathrm{G}$

García-Marín, M., Colina, L., Arribas, S. 2009a, A\&A, 505, 1319

García-Marín, M., Colina, L., Arribas, S. 2009b, A\&A, 505, 1017

Genzel, R., Lutz, D., Sturm, E., et al. 1998, ApJ, 498, 2, 579

Genzel, R., Föerster-Schreiber, N. M., Rosario, D., et al. 2014, ApJ, 796, 7

Harrison, C. M., Alexander, D. M., Mullaney, J. R., Swinbank, A. M. 2014, MNRAS, 441, 3306

Heckman, T. M., Armus, L., \& Miley, G. K. 1990, ApJS, 74, 833

Heckman, T.M., Kauffmann, G., Brinchmann, J., et al., 2004, ApJ, 613, 109H

Hopkins, P. F., Cox, T. J., Younger, J. D., \& Hernquist, L. 2009a, ApJ, 691, 1168

Hopkins, P. F., Somerville, R. S., Cox, T. J., et al. 2009b, MNRAS, 397, 802

Hopkins, P. F., Quataert, E., Murray, N. 2012, MNRAS, 421, 3522

Hopkins, P. F., Torrey, P., Faucher-Giguere, C., et al. 2016, MNRAS, 458, 816

Hung, C.-L., Sanders, D. B., Casey, C. M., et al. 2013, ApJ, 791, 63

Husemann, B., Scharwächter, J., Davis, T. A., et al. 2019, A\&A, 627, 53

Husser, T., Kamann, S., Dreizler, S., et al. 2016, A\&A, 588, 148

Kakkad, D., Mainieri, V., Vietri, G., et al., 2020, A\&A, 642, 147

Kartaltepe, J. S., Dickinson, M., Alexander, D. M., et al. 2012, ApJ, 575, 23

Kauffman, G., Heckman, T. M., Tremonti, C., et al 2003, MNRAS, 346, 1055

Kaviraj, S., Rowlands, K., Alpaslan, M., et al. 2013, MNRAS, 435, 1463

Kewley, L. J., Dopita, M. A., Sutherland, R. S., et al. 2001, ApJ, 556, 121

Kewley, L. J., Groves, B., Kauffmann, G., Heckman, T. 2006, MNRAS, 372, 961

Kewley, L. J., Maier, C., Yabe, K., et al. 2013, ApJ, 774, L10

Kim, D.-C., \& Sanders, D. B. 1998, ApJS, 119, 41

Kim, D.-C., Evans, A. S., Vavilkin, T., et al. 2013, ApJ, 768, 102

Kormendy, J. \& Ho, L. C. 2013, ARAA, 51, 511

Kronberger, T., Kapferer, W., Schindler, S., Ziegler, B. L. 2007, A\&A, 473, 76

Lister, M. L., Kellermann, K. I., Vermeulen, R. C. 2003, ApJ, 584, 135

Lutz, D., Veilleux, S., Genzel, R., 1999, ApJ, 517, 13
Madau, P., Dickinson, M. 2014, ARA\&A, 52, 415

Magnelli, B., Popesso, P., Berta, S., et al. 2013, A\&A, 553, A132 Maiolino, R., Gallerani, S., Neri, R., et al. 2012, MNRAS,425, L66 Maiolino, R. \& Mannucci, F. 2019, A\&ARv, 27, 3

Martin, C. L. 2005, ApJ, 621, 227

Medling, A. M., U, V., Guedes, J., et al. 2014, ApJ, 784, 70

Mingozzi, M., Cresci, G., Venturi, G., et al. 2019, A\&A, 622, 146

Naab, T., Ostriker, J. P. 2017, ARA\&A, 55, 59N

Nagao, T., Marconi, A., \& Maiolino, R. 2006, A\&A, 447, 157

Nardini, E., Risaliti, G., Watabe, Y., et al. 2010, MNRAS, 405, 2505

Nedelchev, B., Sarzi, M., Kaviraj, S. 2019, MNRAS, 486, 1608

Ocvirk, P., Pichon, C., Teyssier, R. 2008, MNRAS, 390, 1326

Osterbrock, D. E. \& Ferland, G. J., 2006, Astrophysics of Gaseous Nebulae and Active Galactic Nuclei. University Science Books

Pereira-Santaella, M., Colina, L., García-Burillo, S. et al. 2018, A\&A, 616, 171

Pérez-González, P. G., Rieke, G. H.; Egami, E. et al. 2005, ApJ, 630, 82P

Perna, M., Lanzuisi, G., Brusa, M., Mignoli, M., Cresci, G., 2017, A\&A, 603A, 99P

Perna, M., Cresci, G., Brusa, M., et al. 2019, A\&A, 623, 171

Perna, M., Arribas, S., Catalán-Torrecilla, C., et al. 2020, A\&A, 643, 139

Petric, A. O., Ho, L. C., Flagey, N. J. M., Scoville, N. Z. 2015, ApJS, 219, 22

Pillepich, A., Springel, V., Nelson, D., et al. 2018, MNRAS, 473, 4077

Rich, J. A., Kewley, L. J. \& Dopita, M. A. 2011, ApJ, 734, 87

Rich, J. A., Kewley, L. J. \& Dopita, M. A. 2015, ApJS, 221, 28

Rodríguez Zaurín, J., Tadhunter, C. N., Rose, M., \& Holt, J. 2013, MNRAS, 432, 138

Rodríguez del Pino, B., Arribas, S., Piqueras López, J., et al., 2019, A\&A, 630, 124

Rothberg, B., \& Joseph, R. D., 2004, AJ, 128, 2098

Rupke, D. S., Veilleux, S., \& Sanders, D. B. 2002, ApJ, 570, 588

Rupke, D.S., Veilleux, S. \& Sanders, D.B. 2005a, ApJS, 160, 87R

Rupke, D.S., Veilleux, S. \& Sanders, D.B. 2005b, ApJS, 160, 115R

Rupke, D.S., Veilleux, S. \& Sanders, D.B. 2005c, ApJ, 632, 751

Rupke, D. S. N., \& Veilleux, S. 2013, ApJ, 775, 15R

Rupke, D. S. N., \& Veilleux, S. 2013, ApJ, 768, 75R

Rupke, D. S. N., \& Veilleux, S. 2015, ApJ, 801, 126

Rupke, D. S. N., Gultekin, K., Veilleux, S. 2017, ApJ, 850, 40

Sanders, D. B., Soifer, B. T., Elias, J. H., et al. 1988, ApJ, 325, 74S

Sanders, D. B., Mazzarella, J. M., Kim, D. C., et al. 2003, AJ, 126, 1607

Sánchez Almeida, J., Elmegreen, B. G., Muñoz-Tuñón, C., Elmegreen, D. M. 2014, A\&ARv, 22, 71

Sato, T., Martin, C. L., Noeske, K. G., et al. 2009, ApJ, 696, 214

Schaye, J., Dalla Vecchia, C., Booth, C.M. et al. 2010, MNRAS, 402, 1536

Scharwächter, J., Eckart, A., Pfalzner, S. et al. 2007, A\&A, 469, 913

Schwarz, G., 1978, Ann. Stat., 6, 461

Smits, D. P. 1996, MNRAS, 278, 683

Silk, J. 2013, ApJ, 772, 112S

Soto, K. T., Lilly, S. J., Bacon, R., et al. 2016, MNRAS, 458, 3210

Spoon, H. W. W., Farrah, D., Lebouteilleur, V., et al. 2013, ApJ, 775, 127

Somerville, R. S., \& Davé, R. 2015, ARA\&A, 53, 51S

Sturm, E., González-Alfonso, E., Veilleux, S., et al. 2011, ApJ, 733, L16

Valdes, F., Gupta, R., Rose, J.A., et al. 2004, ApJS, 152, 251

Veilleux, S., Kim, D.-C., \& Sanders, D. B. 2002, ApJS, 143, 315

Veilleux, S., Cecil, G., Bland-Hawthorn, J. 2005, ARAA, 43, 769

Veilleux, S., Rupke, D. S. N., Kim, D.-C., et al. 2009, ApJS, 182, 628

Veilleux, S., Meléndez, M., Sturm, E., et al. 2013, ApJ, 776,27V

Veilleux, S., Bolatto, A., Tombesi, F., et al. 2017, ApJ, 843, 18V

Veilleux, S., Maiolino, R., Bolatto, A. D., Aalto, S. 2020, A\&ARv, 28, 2

Venturi, G., Nardini, E., Marconi, A., et al. 2018, A\&A, 619, 74

Véron-Cetty, M. -P., Joly, M., Véron, P., 2004, A\&A, 417, 515

Villar Martín, M., Perna, M., Humphrey, A., et al. 2020, A\&A, 634, 116

Westmoquette M.S., Clements D.L., Bendo G.J. \& Khan S.A. 2012, MNRAS 424, 416W

Wisnioski, E., Mendel, J. T., Föerster-Schreiber N.M., et al., 2018, ApJ, 855, 97

Woo, J., H., Bae, H., J., Son, D., \& Karouzos, M. 2016, ApJ, 817, 108

Yuan, F., T., Argudo-Fernández, M., Shen, S., et al. 2018, A\&A, 613, 13

Yun, M. S., Reddy, N., A., Condon, J., J., 2001, ApJ, 554, 803 


\section{Appendix A: Spectroscopic redshifts}

Table A.1 display all spectroscopic redshifts we derived by modelling the stellar continuum and line features of nuclear spectra, as well as of ULIRG nearby companions detected in the MUSE data cubes. Coordinates, velocity offsets, and projected distances from the bright nucleus of each ULIRG are also reported.

\section{Appendix B: Seyfert 1 fit analysis}

For the two Sy 1 nuclear spectra, we proceeded in two steps. First, we modelled the continuum with a power law and the iron emission components with the observational templates of Véron-Cetty et al. (2004). This initial fit was performed considering the wavelength ranges $4050-7100 \AA$ (for F01572+0009) and $4450-7100 \AA$ (for Izw1), but masking all other prominent emission lines, namely the BLR Balmer and He lines, as well as the NLR forbidden [O III], [N II], [S II], and [O I] features. We therefore obtained a template for the continuum and iron emission that we used in the second step.

Then, we modelled all remaining emission lines with a combination of Gaussian profiles, as well as broken power-law functions (e.g. Cresci et al. 2015) for the BLR emission components, together with the continuum and iron components (for which we obtained good initial estimates for the fit parameters from the previous step). This analysis is very similar to the one presented in Sect. 6, except for the following aspects. Additional emission lines were detected and included in the fit of Sy 1 spectra: the Balmer $\mathrm{H} \gamma$ and $\mathrm{H} \delta$, the Si II $\lambda \lambda 6347,71$, the He II at $4685 \AA$, and

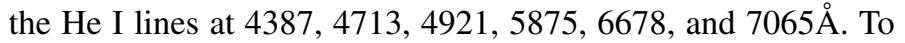
model the Balmer emission lines, we used two parameters for the $\mathrm{H} \alpha$ and $\mathrm{H} \beta$ amplitudes, and derived the $\mathrm{H} \alpha / \mathrm{H} \beta$ flux ratio to measure the dust extinction using the Cardelli et al. (1989) extinction law and assuming case $\mathrm{B}$ recombination as well as $T_{e}=10^{4} \mathrm{~K}$. This dust correction was then used to infer the fluxes of $\mathrm{H} \gamma$ and $\mathrm{H} \delta$, considering case $\mathrm{B}$ recombination flux ratios $\mathrm{H} \gamma / H \beta=0.468$ and $\mathrm{H} \delta / H \beta=0.260$. Similarly, we considered the standard He I theoretical intensity ratios from Smits (1996) to constrain the fluxes of all He I transitions: In particular, we assumed low-density plasma conditions (with $T_{e}=10^{4}$ $\mathrm{K}$ and $\left.N_{e}=10^{3} \mathrm{~cm}^{-3}\right)$ for the NLR component, and high-density conditions (with $T_{e}=10^{4} \mathrm{~K}$ and $N_{e}=10^{6} \mathrm{~cm}^{-3}$ ) for the BLR. Therefore we used a unique parameter for the HeI $\lambda 4471$ flux amplitude, and the theoretical intensity ratios to infer the fluxes of the remaining transitions, correcting for the dust extinction (from the Balmer decrement). For the BLR components, we assumed that all Balmer and helium lines have the same broken power-law indices (e.g. Nagao et al. 2006) and kinematics. BLR emission components were used for all permitted transitions (see e.g. Véron-Cetty et al. 2004).

\section{Appendix C: Individual ULIRGs}

Figures C.1 C.89 display the three-colour composites, stellar and emission line maps, as well as the nuclear spectra for each ULIRG presented in this paper. 
Table A.1. ULIRG nuclei and companion (pPXF spectroscopic) redshifts and positions.

\begin{tabular}{|c|c|c|c|c|c|}
\hline source name & $\left(\begin{array}{c}\mathrm{RA} \\
\left({ }^{h}:{ }^{m}:{ }^{s}\right) \\
(2)\end{array}\right.$ & $\begin{array}{c}\text { DEC } \\
\left({ }^{\circ}:{ }^{\prime}:{ }^{\prime \prime}\right) \\
(3)\end{array}$ & (4) & $\begin{array}{c}\Delta V \\
(\mathrm{~km} / \mathrm{s}) \\
(5)\end{array}$ & $\begin{array}{l}\text { Projected distance } \\
(" / / \mathrm{kpc}) \\
(6)\end{array}$ \\
\hline F00188-0856 & $0: 21: 26.52$ & $-8: 39: 25.92$ & $0.1284 \pm 0.0001$ & & - \\
\hline F00188-0856: c & $0: 21: 25.43$ & $-8: 39: 18.46$ & $0.1219 \pm 0.0001$ & $-60 \pm 25$ & $18.0 / 41.6$ \\
\hline IZw1 & $0: 53: 34.93$ & $+12: 41: 35.94$ & $0.0611 \pm 0.0001$ & - & - \\
\hline IZw1: c & $0: 53: 33.88$ & $+12: 41: 33.63$ & $0.0613 \pm 0.0002$ & $+60 \pm 25$ & $15.5 / 18.4$ \\
\hline F01572+0009 & $1: 59: 50.25$ & $+0: 23: 40.87$ & $0.1632 \pm 0.0001$ & - & - \\
\hline F01572+0009: c & $1: 59: 48.76$ & $+0: 23: 43.49$ & $0.1623 \pm 0.0001$ & $-244 \pm 10$ & $21.7 / 61.3$ \\
\hline F05189-2524 & $5: 21: 01.40$ & $-25: 21: 45.30$ & $0.0428 \pm 0.0001$ & - & - \\
\hline 07251-0248 E & $7: 27: 37.61$ & $-2: 54: 54.25$ & $0.0879 \pm 0.0001$ & - & - \\
\hline 07251-0248 W & $7: 27: 37.54$ & $-2: 54: 54.39$ & $0.0881 \pm 0.0001$ & $55 \pm 10$ & $1.1 / 1.8$ \\
\hline $09022-3615$ & 9:04:12.71 & $-36: 27: 01.93$ & $0.0596 \pm 0.0001$ & - & - \\
\hline $10190+1322 \mathrm{~W}$ & $10: 21: 42.49$ & $+13: 06: 53.83$ & $0.0767 \pm 0.0001$ & - & - \\
\hline $10190+1322 \mathrm{E}$ & $10: 21: 42.75$ & $+13: 06: 55.61$ & $0.0758 \pm 0.0001$ & $-264 \pm 10$ & $4.9 / 7.2$ \\
\hline $10190+1322: \mathrm{c}_{S E}$ & $10: 21: 43.83$ & $+13: 06: 47.54$ & $0.0760 \pm 0.0001$ & $-187 \pm 11$ & $20.6 / 30.1$ \\
\hline $10190+1322: c_{N W}$ & $10: 21: 41.88$ & $+13: 07: 06.36$ & $0.0771 \pm 0.0001$ & $120 \pm 11$ & $15.8 / 23.1$ \\
\hline F11095-0238 NE & $11: 12: 03.38$ & $-2: 54: 22.94$ & $0.1064 \pm 0.0001$ & & - \\
\hline F11095-0238 SW & 11:12:03.36 & $-2: 54: 23.30$ & $0.1065 \pm 0.0002$ & $+30 \pm 18$ & $0.6 / 1.1$ \\
\hline F12072-0444 N & $12: 09: 45.12$ & $-5: 01: 13.31$ & $0.1288 \pm 0.0001$ & - & - \\
\hline F12072-0444 S & $12: 09: 45.13$ & $-5: 01: 14.23$ & $0.1288 \pm 0.0001$ & $-7 \pm 10$ & $1.0 / 2.3$ \\
\hline F12072-0444: $c_{1}$ & 12:09:44.81 & $-5: 00: 54.78$ & $0.1302 \pm 0.0006$ & $360 \pm 30$ & $19.1 / 44.2$ \\
\hline F12072-0444: $c_{2}$ & 12:09:44.82 & $-5: 00: 52.33$ & $0.1302 \pm 0.0006$ & $360 \pm 30$ & $21.6 / 49.94$ \\
\hline $13120-5453$ & $13: 15: 06.32$ & $-55: 09: 22.82$ & $0.0310 \pm 0.0001$ & - & - \\
\hline F13451+1232 W & $13: 47: 33.36$ & $+12: 17: 24.24$ & $0.1218 \pm 0.0002$ & - & - \\
\hline F13451+1232 E & $13: 47: 33.49$ & $+12: 17: 23.76$ & $0.1218 \pm 0.0002$ & $-5 \pm 10$ & $2.0 / 4.3$ \\
\hline F14348-1447 SW & $14: 37: 38.28$ & $-15: 00: 24.24$ & $0.0824 \pm 0.0002$ & - & - \\
\hline F14348-1447 NE & $14: 37: 38.40$ & $-15: 00: 21.29$ & $0.0822 \pm 0.0002$ & $-49 \pm 30$ & $3.4 / 5.3$ \\
\hline F14348-1447: c & $14: 37: 38.95$ & $-15: 00: 25.47$ & $0.0823 \pm 0.0001$ & $-22 \pm 8$ & $10.3 / 16.1$ \\
\hline F14378-3651: $\mathrm{n}$ & $14: 40: 58.89$ & $-37: 04: 32.08$ & $0.0682 \pm 0.0001$ & - & - \\
\hline F14378-3651: c & 14:41:01.15 & $-37: 04: 43.94$ & $0.0684 \pm 0.0001$ & $48 \pm 7$ & $29.7 / 38.9$ \\
\hline F15327+2340 (Arp220): $\mathrm{n}_{W}$ & $15: 34: 57.24$ & $+23: 30: 11.70$ & $0.0181 \pm 0.0001$ & - & - \\
\hline $\mathrm{F} 15327+2340(\operatorname{Arp} 220): \mathrm{n}_{E}$ & $15: 34: 57.30$ & $+23: 30: 11.90$ & $0.0182 \pm 0.0001$ & $30 \pm 9$ & $1 / 0.37$ \\
\hline F16090-0139: n & $16: 11: 40.42$ & $-1: 47: 06.56$ & $0.1337 \pm 0.0002$ & - & - \\
\hline 17208-0014: $\mathrm{n}$ & $17: 23: 21.94$ & $-00: 17: 00.96$ & $0.0430 \pm 0.0001$ & - & - \\
\hline F19297-0406 S & 19:32:22.30 & $-4: 00: 01.80$ & $0.0854 \pm 0.0001$ & - & - \\
\hline F19297-0406 N & $19: 32: 22.31$ & $-4: 00: 01.03$ & $0.0853 \pm 0.0001$ & $-25 \pm 9$ & $0.7 / 1.1$ \\
\hline $19542+1110$ & $19: 54: 35.78$ & $11: 19: 05.03$ & $0.0624 \pm 0.0002$ & - & - \\
\hline 19542+1110: c & 19:54:34.91 & 11:19:20.94 & $0.0628 \pm 0.0001$ & $40 \pm 15$ & $20.5 / 24.8$ \\
\hline $20087-0308$ & $20: 11: 23.87$ & $-2: 59: 50.71$ & $0.1052 \pm 0.0001$ & - & - \\
\hline 20100-4156 SE & $20: 13: 29.56$ & $-41: 47: 35.21$ & $0.1297 \pm 0.0002$ & - & - \\
\hline $20100-4156 \mathrm{NW}$ & $20: 13: 29.48$ & $-41: 47: 32.58$ & $0.1297 \pm 0.0001$ & $-6 \pm 10$ & $2.8 / 6.5$ \\
\hline $20100-4156$ c & $20: 13: 28.63$ & $-41: 47: 38.39$ & $0.1300 \pm 0.0001$ & $90 \pm 8$ & $10.5 / 24.3$ \\
\hline F22491-1808 W & $22: 51: 49.24$ & $-17: 52: 23.66$ & $0.0776 \pm 0.0001$ & - & - \\
\hline F22491-1808 E & $22: 51: 49.35$ & $-17: 52: 24.12$ & $0.0777 \pm 0.0002$ & $+35 \pm 9$ & $1.8 / 2.7$ \\
\hline
\end{tabular}

Notes. Column (1): Target name. (2) and (3): Coordinates (RA and DEC). (4): Spectroscopic redshift from pPXF at the position of the stellar continuum peak. (5): Velocity with respect to the ULIRG systemic. (6): Projected distance from the ULIRG (brightest) nucleus. 

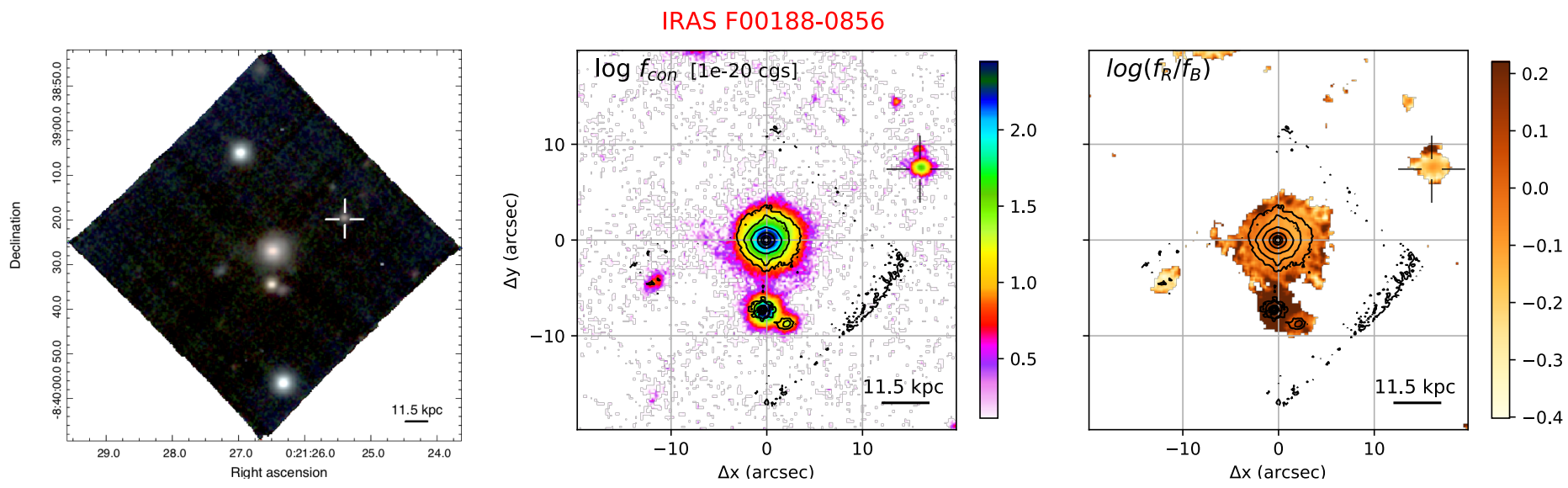

Fig. C.1. IRAS F00188-0856 images from MUSE observations with TOT $=0.68 \mathrm{hr}$. Left: Colour-composite optical image, showing [O III] (green, from the wavelength range $4990-5015 \AA$ rest-frame), $\mathrm{H} \alpha$ (red, $6555-6574 \AA$ ), and stellar continuum (blue, $4400-4500 \AA$ ). Centre: Red $(7390-7530 \AA)$ continuum image, with contours from HST/F160W. Right: Continuum colour map obtained from MUSE by dividing the red continuum image (in the central panel) by a blue image obtained by collapsing the stellar emission in the range $4400-4500 \AA$; contours from HST/F160W. In all panels, we display the IRAS F00188-0856 companion with a cross.
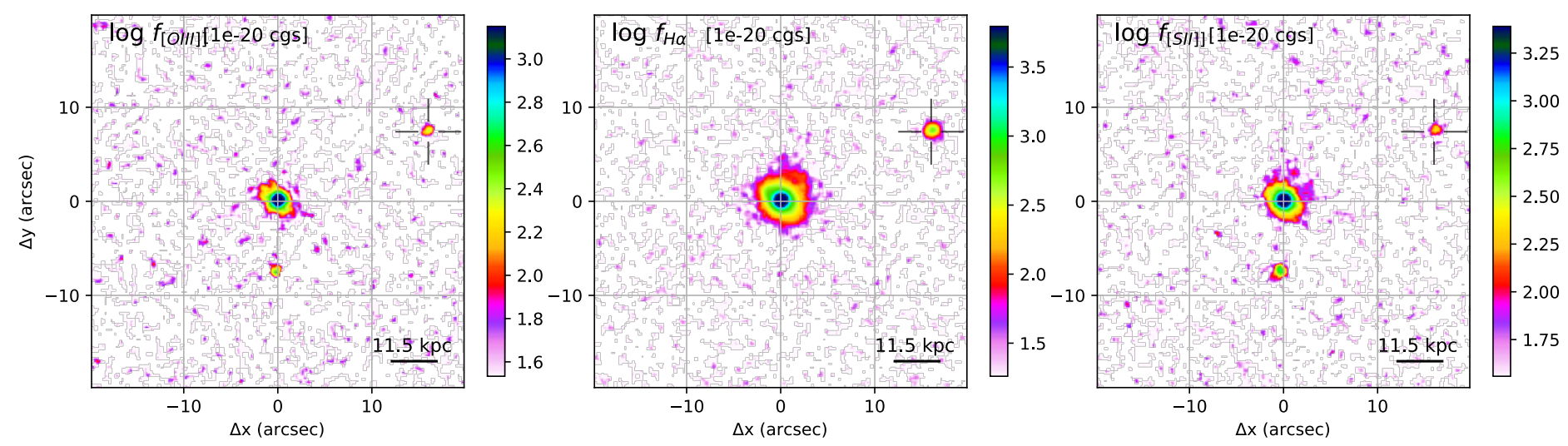

Fig. C.2. IRAS F00188-0856 emission line images from MUSE observations. [O III] (left, from the wavelength range $4990-5015 \AA ̊$ rest-frame), $\mathrm{H} \alpha$ (centre, $6555-6574 \AA$ ), and [S II] (right, $6702-6742 \AA$ ) images have been obtained by subtracting continuum emission using the adjacent regions at shorter and longer wavelengths with respect to the emission line systemics. In all panels, we display the position of the companion galaxy with a cross.
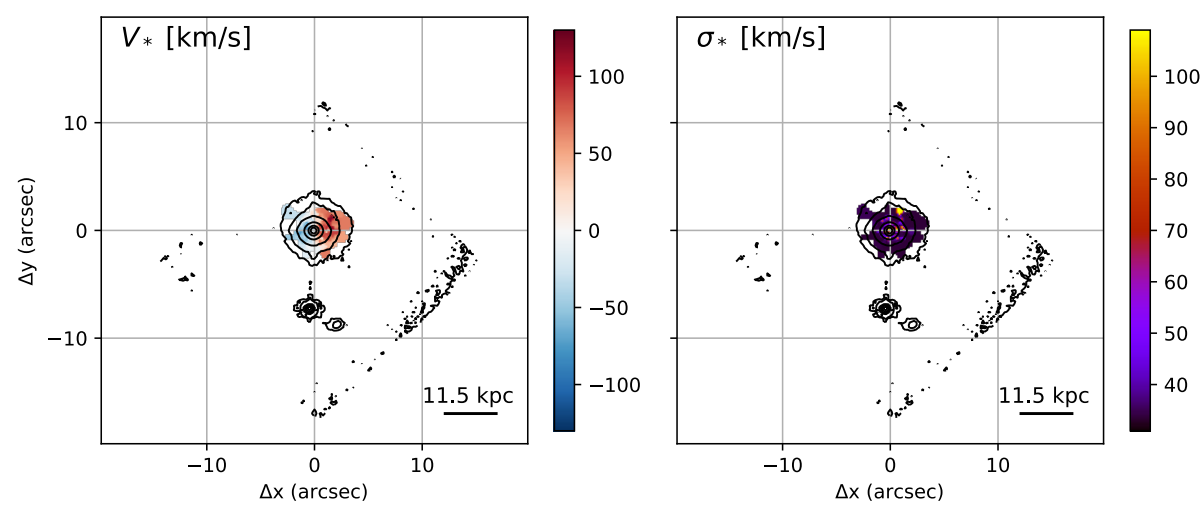

Fig. C.3. IRAS F00188-0856 stellar kinematic maps from the pPXF analysis with contours from HST/F160W. The left panel shows the stellar velocity $V_{*}$, and the right panel displays the velocity dispersion $\sigma_{*}$. 


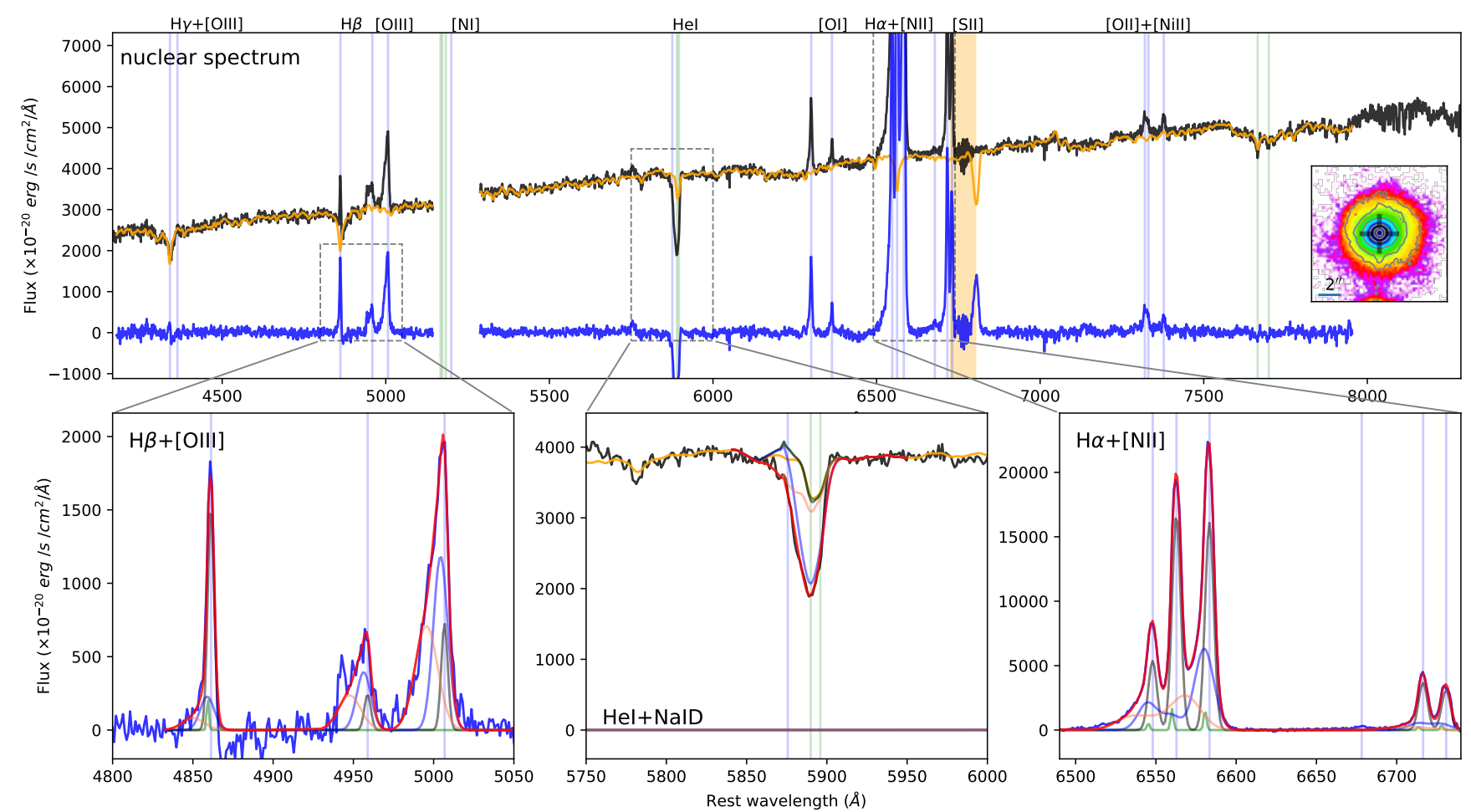

Fig. C.4. Top panel: IRAS F00188-0856 nuclear spectrum (black curve), extracted from a circular aperture with $r<0.4^{\prime \prime}$. The corresponding pPXF best-fit model profile is shown in orange. The continuum-subtracted spectrum (blue curve) is obtained by subtracting the best-fit pPXF model from the original spectrum. The vertical blue lines mark the wavelengths of the emission lines detected in the spectrum; the vertical green lines mark the position of stellar absorption systems (i.e. from left to right: MgI triplet, $\mathrm{Na}$ ID and KI doublets). The regions excluded from the pPXF fit and corresponding to the most intense sky line residuals are highlighted as orange shaded areas; the portions of the spectra around $5300 \AA$ are missing because a filter blocked the laser contamination. The inset in the top panel shows the red stellar continuum emission map in the vicinity of the nuclear regions, with HST/F160W contours as in Fig. C.1. the nuclear position is shown with a black marker. Bottom insets: Multi-component best-fit analysis results for the main emission (H $\beta$ and [O III], left; $\mathrm{H} \alpha$, [N II] and [S II], right) and absorption (Na ID, centre) line features. Na ID multi-component best-fit curves are superimposed on the observed spectrum (black curve); emission components are not required to reproduce the total profile of Na ID. 

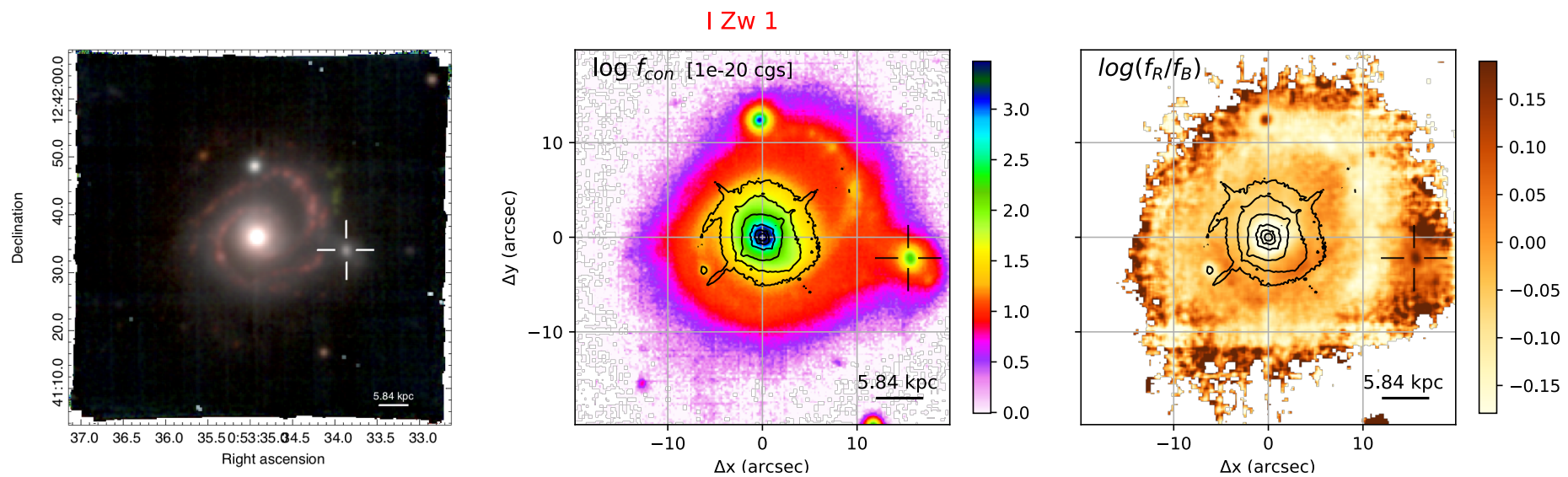

Fig. C.5. IZw1 images from MUSE observations with TOT $=2.72 \mathrm{hr}$. Left: Colour-composite optical image, showing [O III] (green, from the wavelength range $4959-5018 \AA$ rest-frame), H $\alpha$ (red, $6552-6570 \AA$ ), and stellar continuum (blue, $4570-4670 \AA$ ). Centre: Red (8010 $8110 \AA$ ) continuum image from MUSE, with contours from HST/F160W. Right: Continuum colour map obtained from MUSE by dividing the red continuum image (central panel) by a blue image obtained by collapsing the stellar emission in the range $4570-4670 \AA$; contours from HST/F160W. In all panels, we display the IZw1 companion galaxy with a cross.
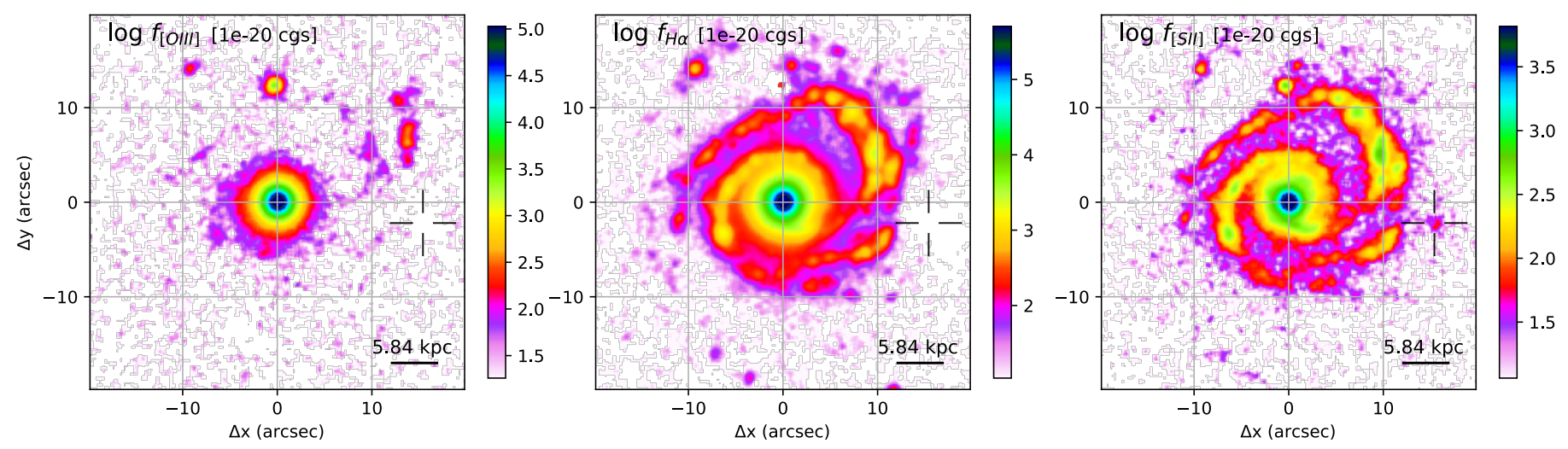

Fig. C.6. IZw1 emission line images from MUSE observations. [O III] (left, from the wavelength range $4985-5018 \AA ̊$ rest-frame), H $\alpha$ (centre, 6552-6570 ) and [S II] (right, 6710-6741 ) images have been obtained by subtracting continuum emission using the adjacent regions at shorter and longer wavelengths with respect to the emission line systemics. In all panels, we display the position of the non-emission line companion galaxy of IZw1 with crosses.
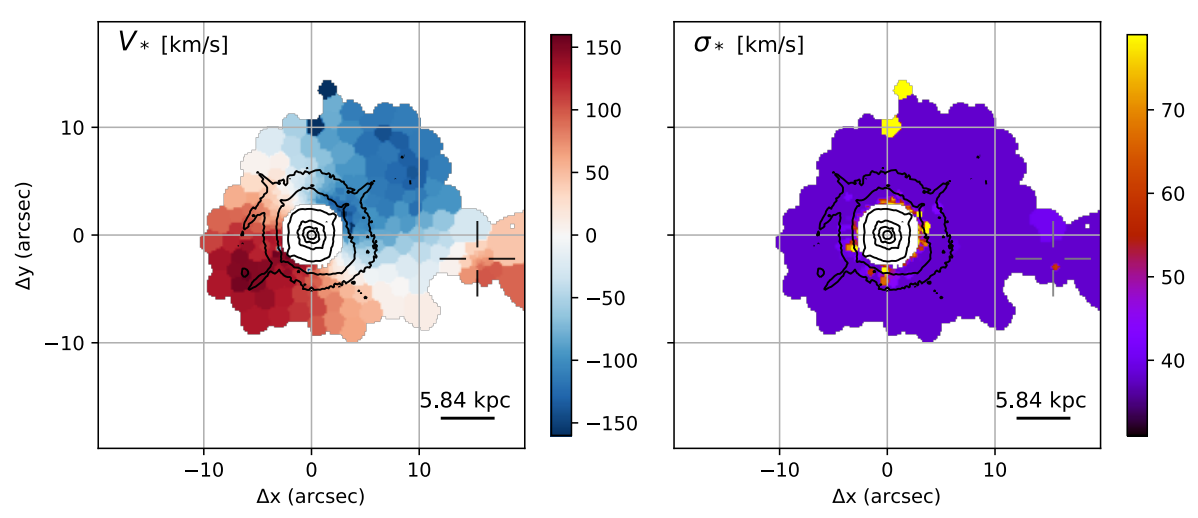

Fig. C.7. Left: IZw1 stellar kinematic maps from the pPXF analysis with contours from HST/F160W. The left panel shows the stellar velocity $V_{*}$, and the right panel represents the velocity dispersion $\sigma_{*}$. The central pixels have been masked because of strong AGN continuum and BLR emission, which prevents the detection of stellar continuum and absorption features. 

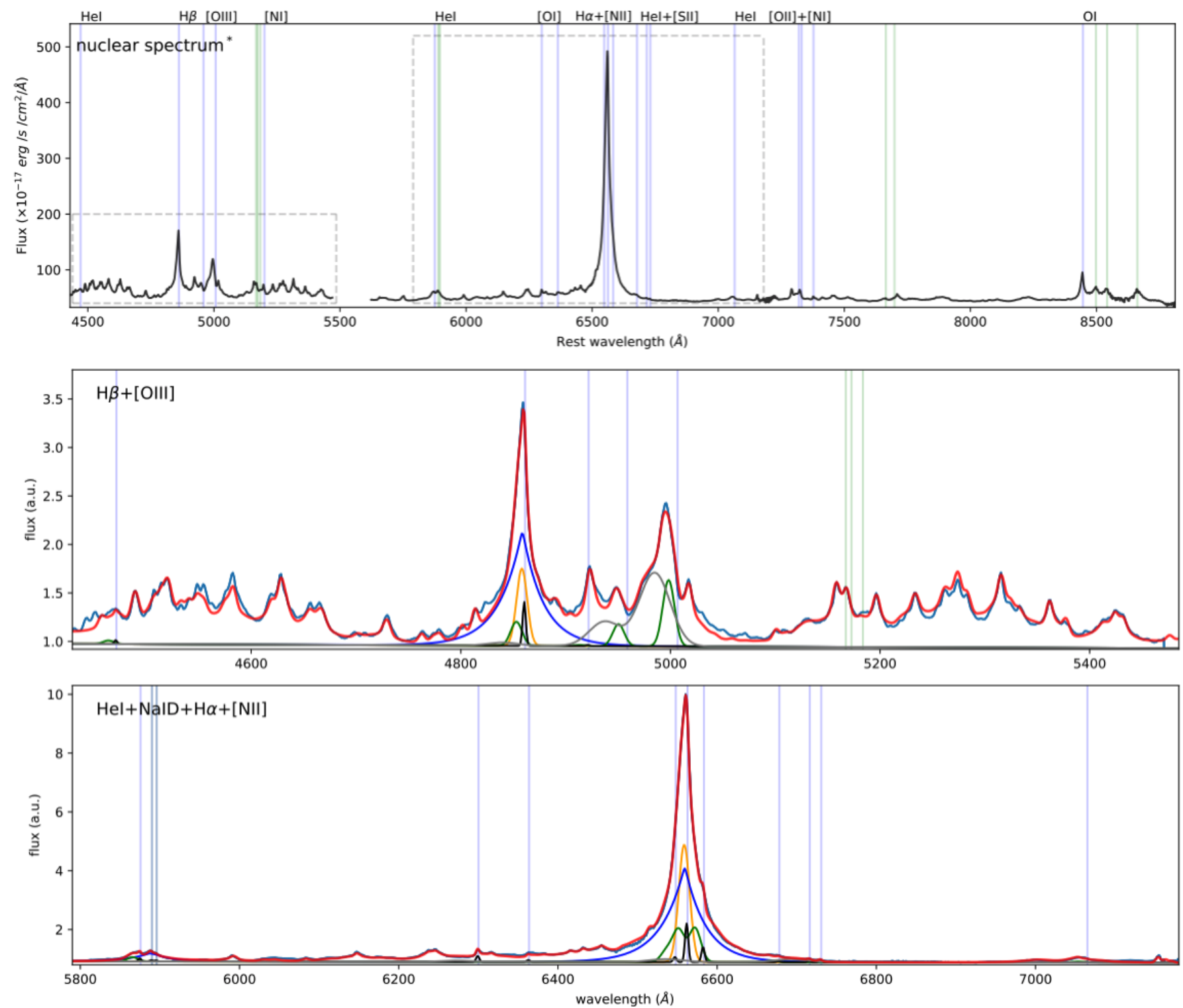

Fig. C.8. Top: IZw1 nuclear spectrum (black curve) extracted from a circular aperture $r<0.4^{\prime \prime}$ but excluding the central pixel (where H $\alpha$ is saturated). The blue vertical lines mark the wavelengths of the emission lines detected in the spectrum; the green lines mark the position of stellar absorption systems (i.e. from left to right: $\mathrm{MgI}$ triplet, $\mathrm{Na}$ ID and $\mathrm{KI}$ doublets, CaII triplet). The latter transitions in absorption are not detected because of the strong AGN continuum and emission lines. The portions of the spectra around $5500 \AA$ are missing because a filter blocked the laser contamination; the portions in the two boxes are reported in the central and bottom panels with the best-fit curves. Centre: Normalised nuclear spectrum in the vicinity of the $\mathrm{H} \beta+[\mathrm{O} \mathrm{III}]$ complex (blue curve), with total best-fit model (red). Individual kinematic components are shown with different colours: black, green, and grey Gaussians represent systemic and outflow components; blue and orange curves are used to model the BLR Balmer emission; and iron components are not reported. Bottom: Normalised nuclear spectrum in the vicinity of the HeI+Na ID and H $\alpha+\mathrm{NII}$ complexes (blue curve), with superimposed best-fit model (red curve). Different kinematic components required to reproduce the spectrum are shown as in the central panel. 

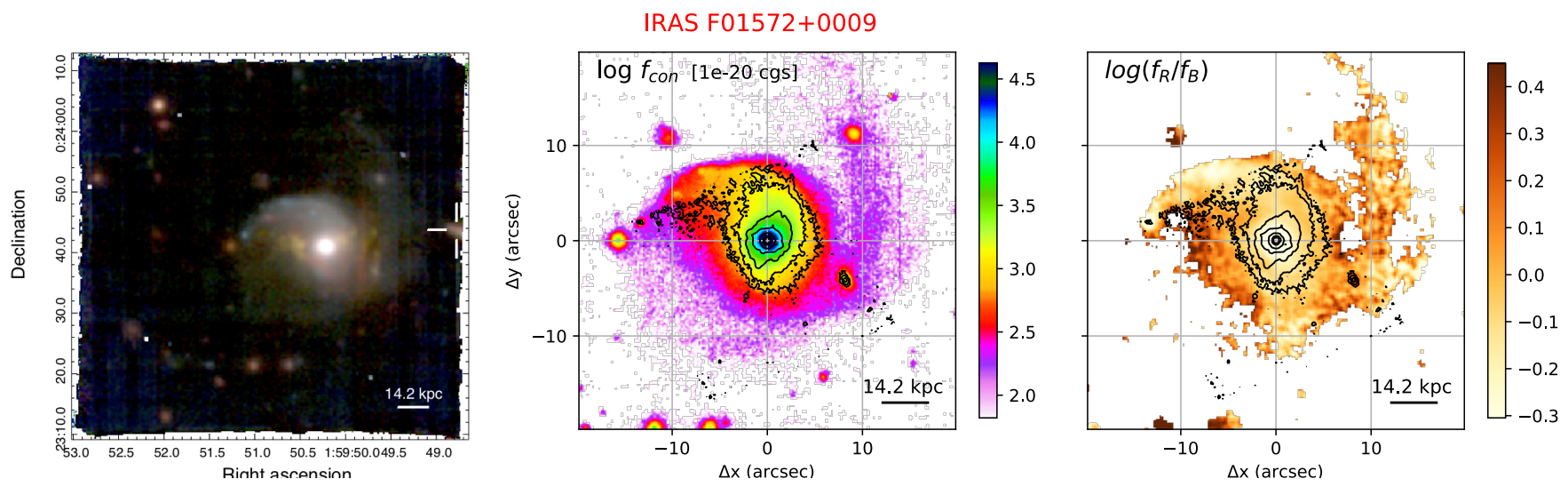

Fig. C.9. IRAS F01572+0009 images from MUSE observations with TOT = $2.04 \mathrm{hr}$. Left: Colour-composite optical image showing [O III] (green, from the wavelength range $4925-4970 \AA$ rest-frame), $\mathrm{H} \alpha$ (red, $6550-6575 \AA$ ), and continuum (blue, $4150-4220 \AA$ ). Centre: Red (7330-7400 $)$ continuum image from MUSE with contours from HST/F160W. Right: Continuum colour map obtained from MUSE by dividing the red continuum image (central panel) by a blue image obtained by collapsing the stellar emission in the range $4150-4220 \AA$; contours from HST/F160W. In the first panel, we display the F01572+0009 companion galaxy with a cross.
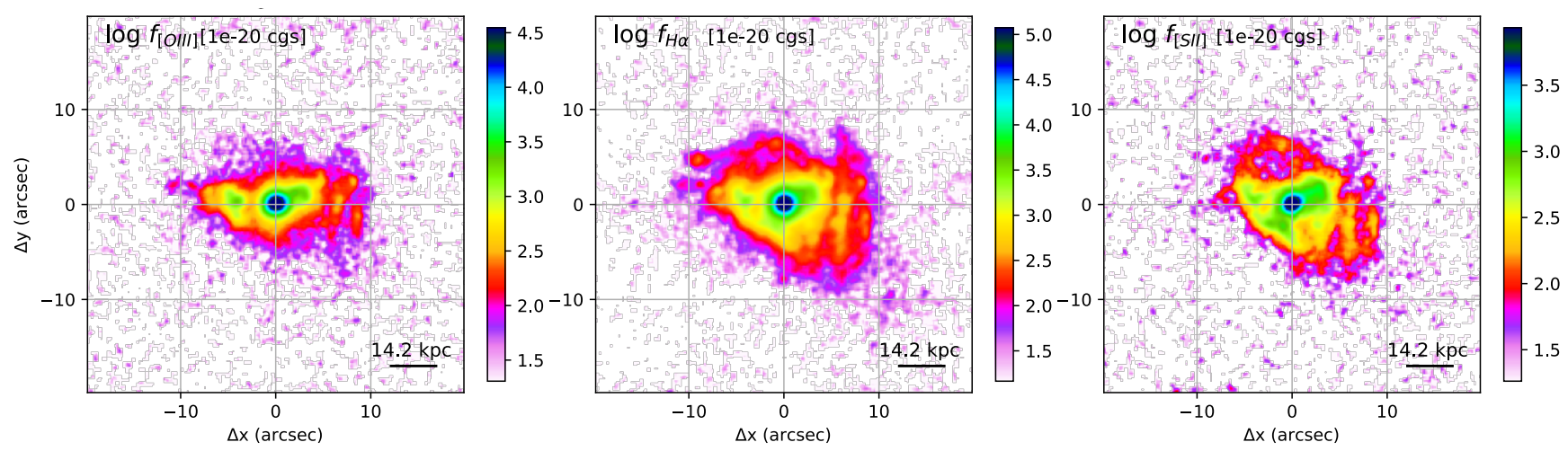

Fig. C.10. IRAS F01572+0009 emission line images from MUSE observations. [O III] (left, from the wavelength range 4925 - 4970 ̊ rest-frame), $\mathrm{H} \alpha$ (centre, $6550-6575 \AA$ ), and [S II] (right, $6700-6748 \AA$ ) images have been obtained by subtracting continuum emission using the adjacent regions at shorter and longer wavelengths with respect to the emission line systemics.
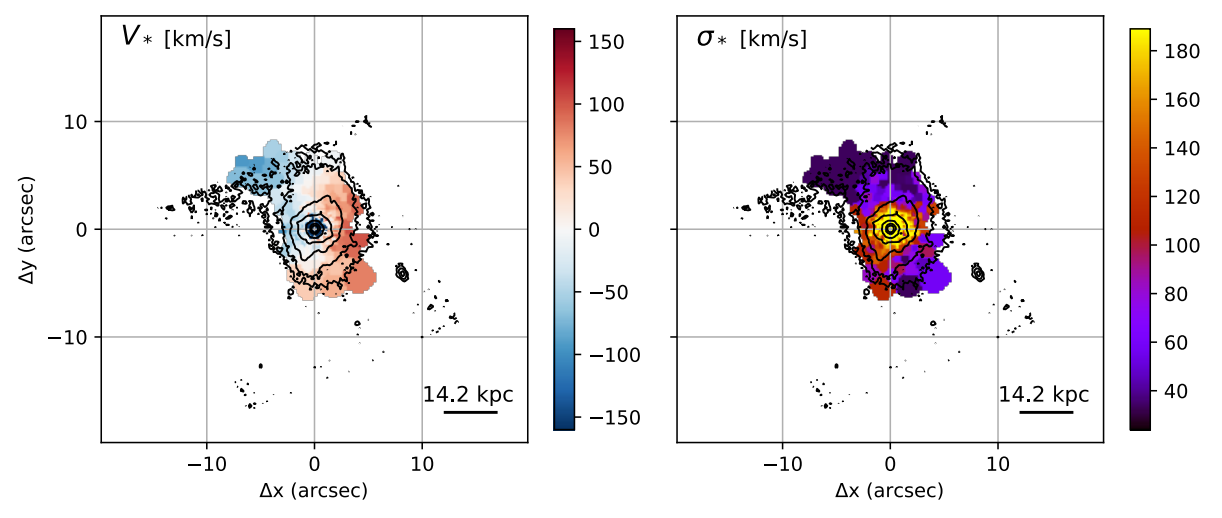

Fig. C.11. Left: IRAS F01572+0009 stellar kinematic maps from the pPXF analysis with contours from HST/F160W. The left panel shows the stellar velocity $V_{*}$, and the right panel represents the velocity dispersion $\sigma_{*}$. 

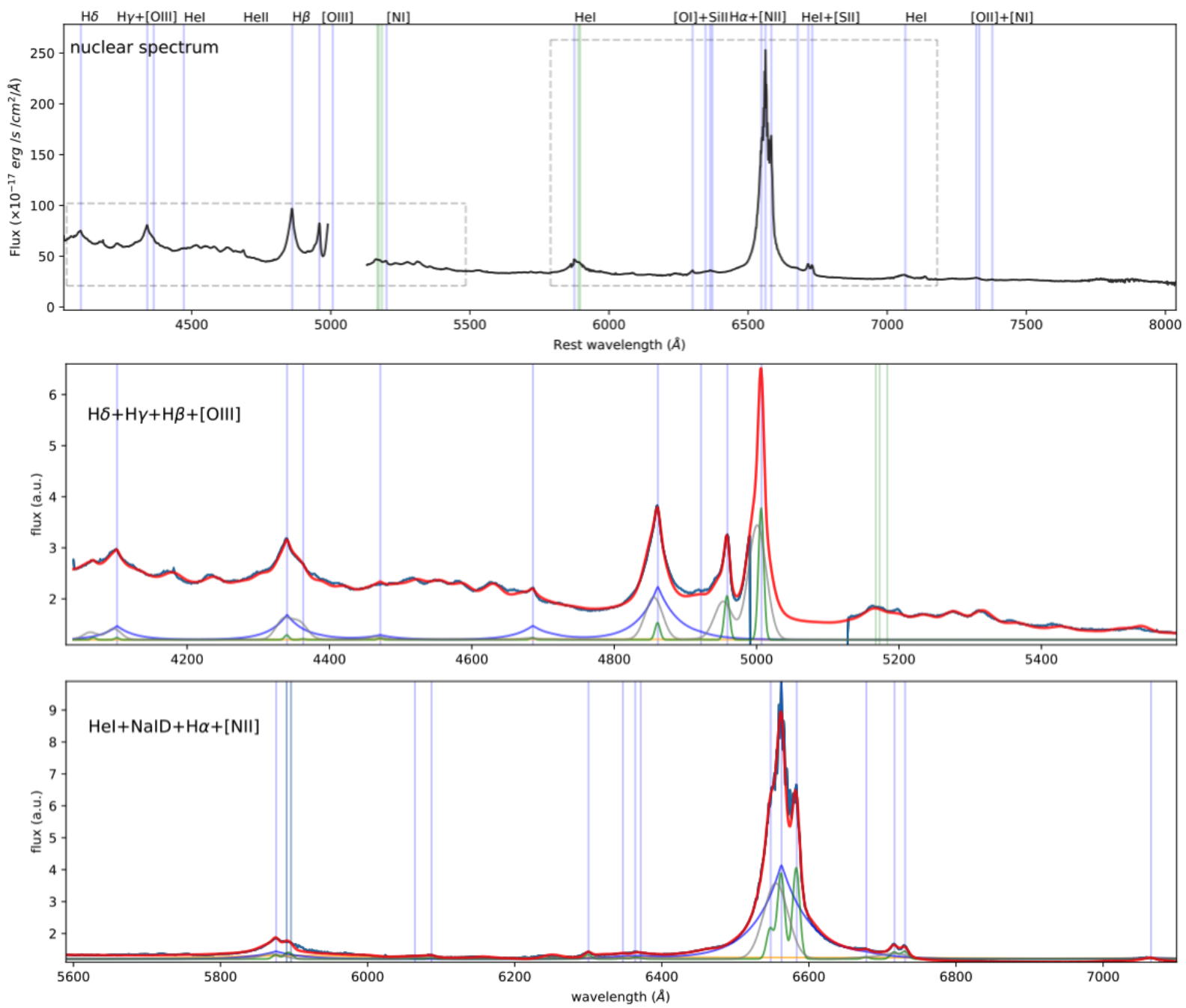

Fig. C.12. Top: IRAS F01572+0009 nuclear spectrum (black curve) extracted from a circular aperture $r<0.4^{\prime \prime}$. The blue vertical lines mark the wavelengths of the emission lines detected in the spectrum; the green lines mark the position of stellar absorption systems (i.e. from left to right: $\mathrm{MgI}$ triplet, and Na ID doublets). The latter transitions in absorption are not detected because of the strong AGN continuum and emission lines. The portions of the spectra around $5050 \AA$ are missing because a filter blocked the laser contamination; the portions in the two boxes are reported in the central and bottom panels with the best-fit curves. Centre: Normalised nuclear spectrum in the vicinity of the H $\beta+[\mathrm{O}$ III] complex (blue curve), with total best-fit model (red). Individual kinematic components are shown with different colours: green and grey Gaussians represent systemic and outflow components, respectively; blue and orange curves are used to model the BLR Balmer emission; and iron components are not reported. The [O III] 55007 line is mostly within the missing portion of the spectrum; this profile has been reconstructed using the [O III] $\lambda 4959$ transition. Bottom: Normalised nuclear spectrum in the vicinity of the HeI+Na ID and $\mathrm{H} \alpha+\mathrm{NII}$ complexes (blue curve), the best-fit model is superimposed (red curve). The different kinematic components required to reproduce the spectrum are shown as in the central panel. 

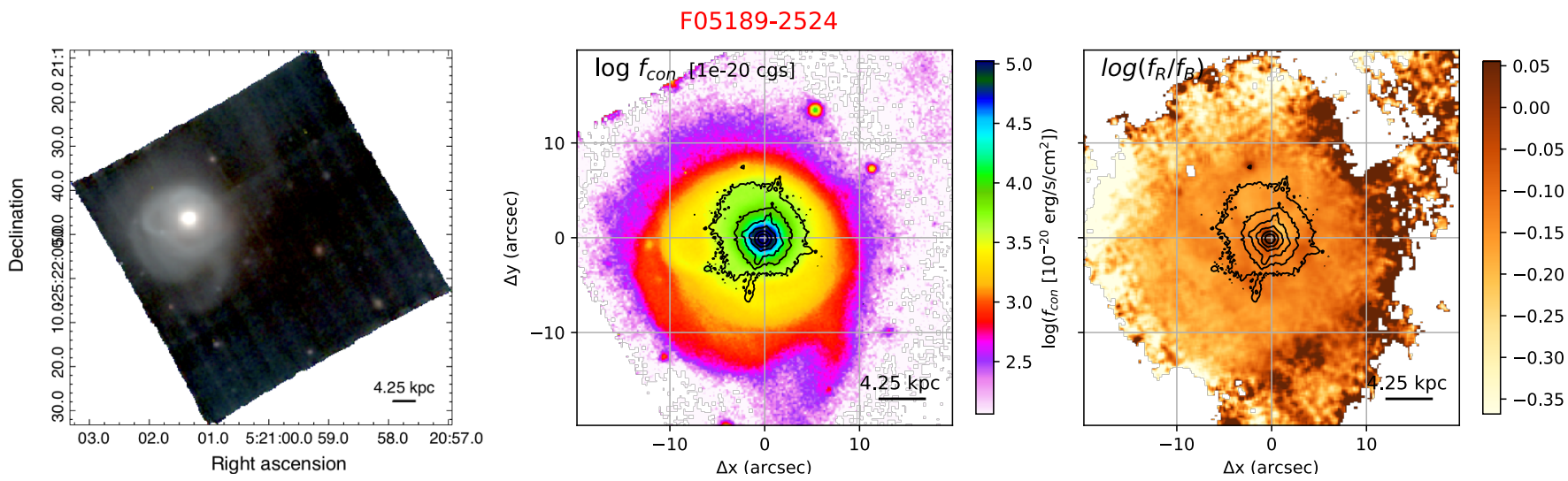

Fig. C.13. IRAS F05189-2524 images from MUSE observations with TOT = 2.04 hr. Left: Colour-composite optical image, showing [O III] (green, from the wavelength range $4962-5022 \AA$ rest-frame), $\mathrm{H} \alpha$ (red, $6554-6578 \AA$ ), and continuum (blue, $4530-4660 \AA$ ) emission. Centre: Red $(8025-8150 \AA$ ) continuum image from MUSE with contours from HST/F160W. Right: Continuum colour map obtained from MUSE by dividing the red continuum image (central panel) by a blue image obtained by collapsing the emission in the range 4530 - $4660 \AA$; contours from HST/F160W.
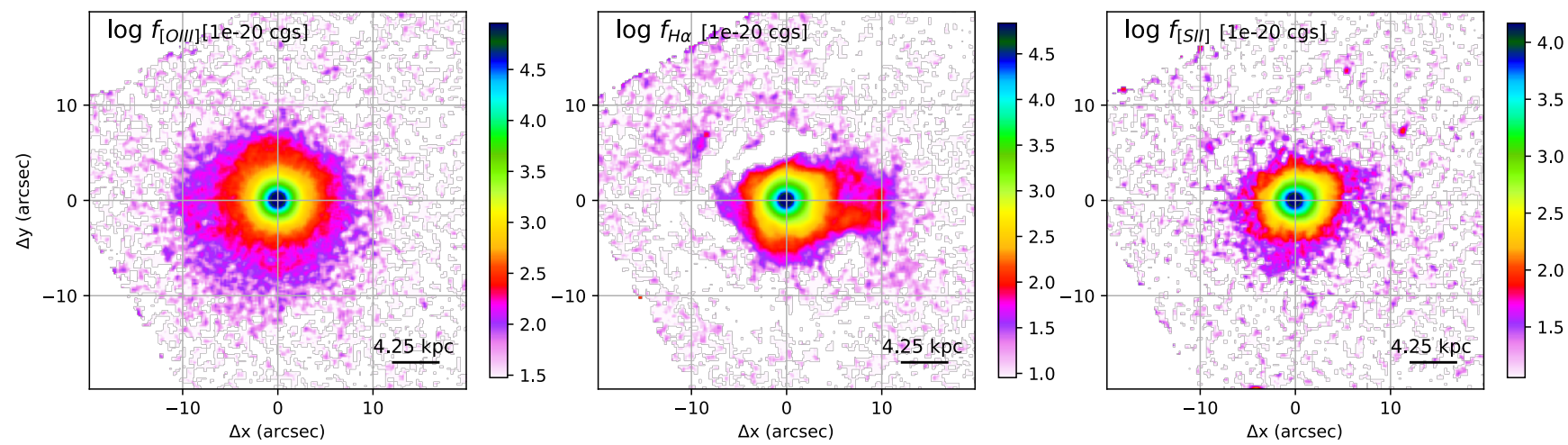

Fig. C.14. IRAS F05189-2524 emission line images from MUSE observations. [O III] (left, from the wavelength range $4962-5022 \AA$ rest-frame), $\mathrm{H} \alpha$ (centre, $6554-6578 \AA$ ), and [S II] (right, $6694-6742 \AA$ ) images have been obtained by subtracting continuum emission using the adjacent regions at shorter and longer wavelengths with respect to the emission line systemics.
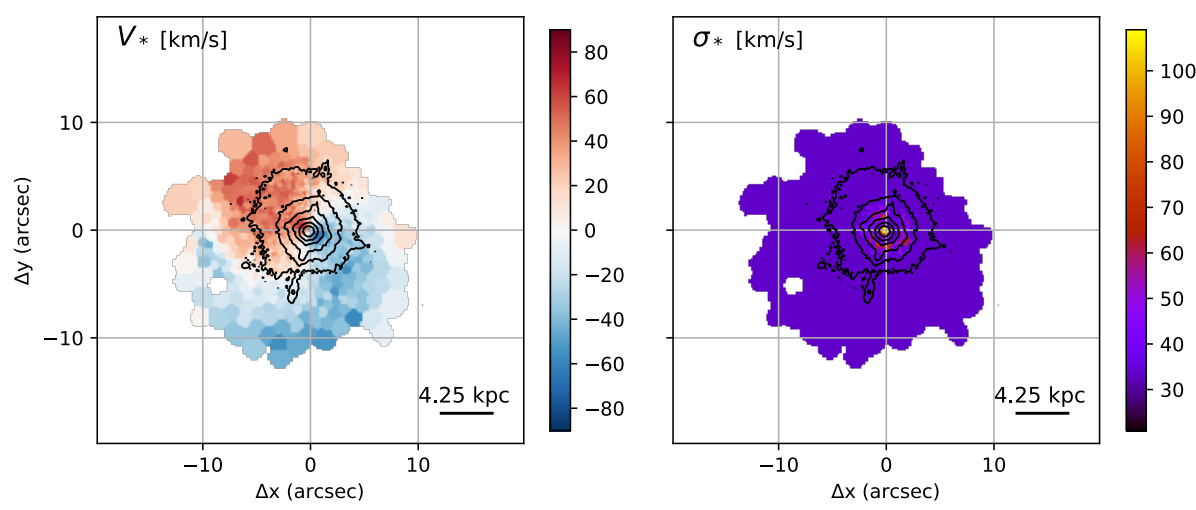

Fig. C.15. Left: IRAS F05189-2524 stellar kinematic maps from the pPXF analysis with contours from HST/F160W. The left panel shows the stellar velocity $V_{*}$, and the right panel represents the velocity dispersion $\sigma_{*}$. 


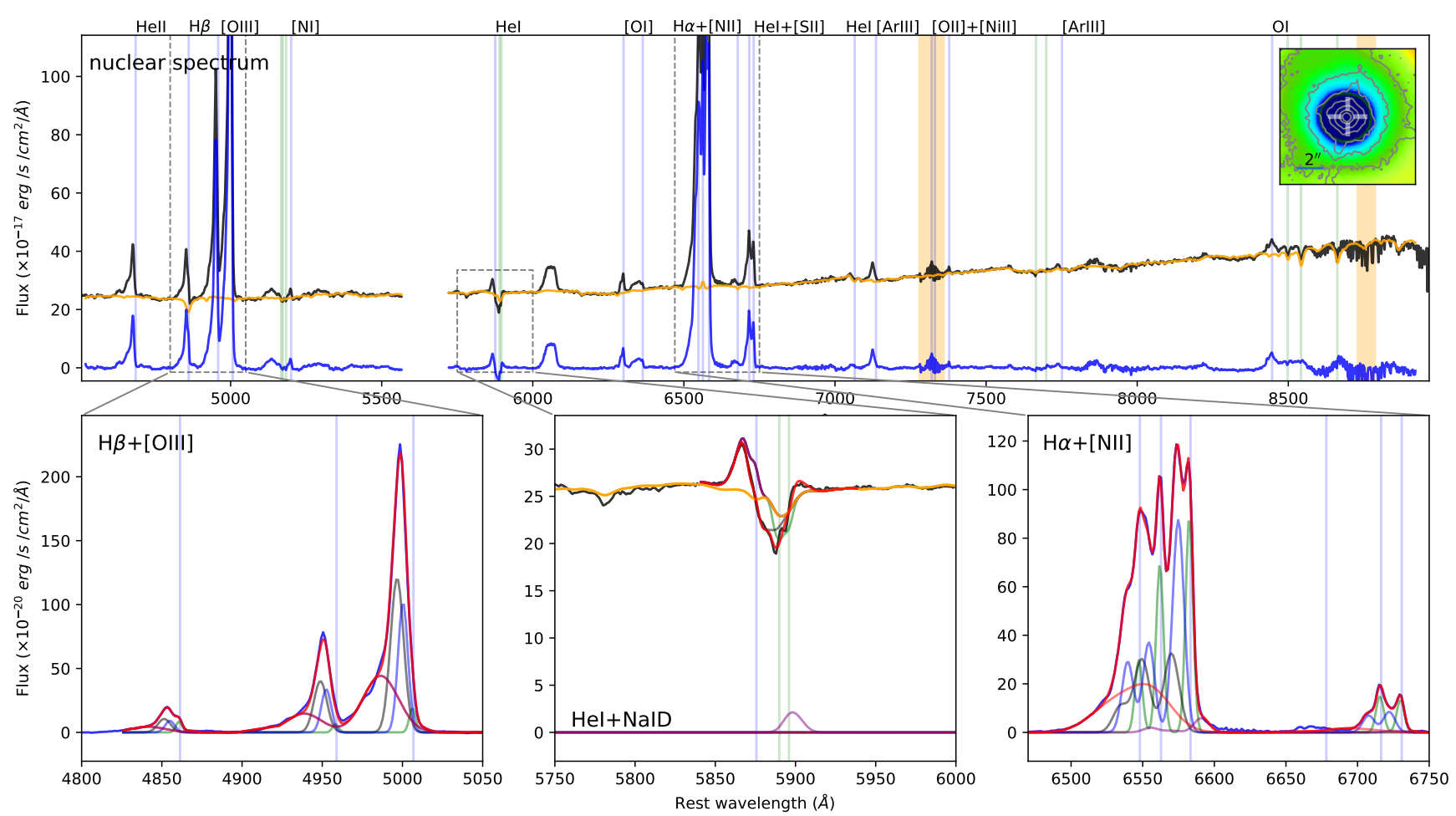

Fig. C.16. Top panel: IRAS F05189-2524 nuclear spectrum (black curve) extracted from a circular aperture with $r<0.4^{\prime \prime}$. The corresponding pPXF best-fit model profile is shown in orange. The continuum-subtracted spectrum (blue curve) is obtained by subtracting the best-fit pPXF model from the original spectrum. The vertical blue lines mark the wavelengths of the emission lines detected in the spectrum, and the vertical green lines mark the position of stellar absorption systems (i.e. from left to right: MgI triplet, $\mathrm{Na}$ ID and KI doublets, CaII triplet). The regions excluded from the pPXF fit and corresponding to the most intense sky line residuals are highlighted as orange shaded areas; the portions of the spectra around $5700 \AA$ are missing because a filter blocked the laser contamination. The inset in the top panel shows the red stellar continuum emission map in the vicinity of the nuclear regions with HST/F160W contours as in Fig. C.13, the nuclear position is shown with a white marker. Bottom insets: Multi-component best-fit analysis results for the main emission (H $\beta$ and [O III], left; $\mathrm{H} \alpha$, [N II] and [S II], right) and absorption (Na ID, centre) line features. Na ID multi-component best-fit curves required to reproduce the cool, neutral absorption are superimposed on the observed spectrum (black curve); emission components are shifted downward for the sake of clarity. 

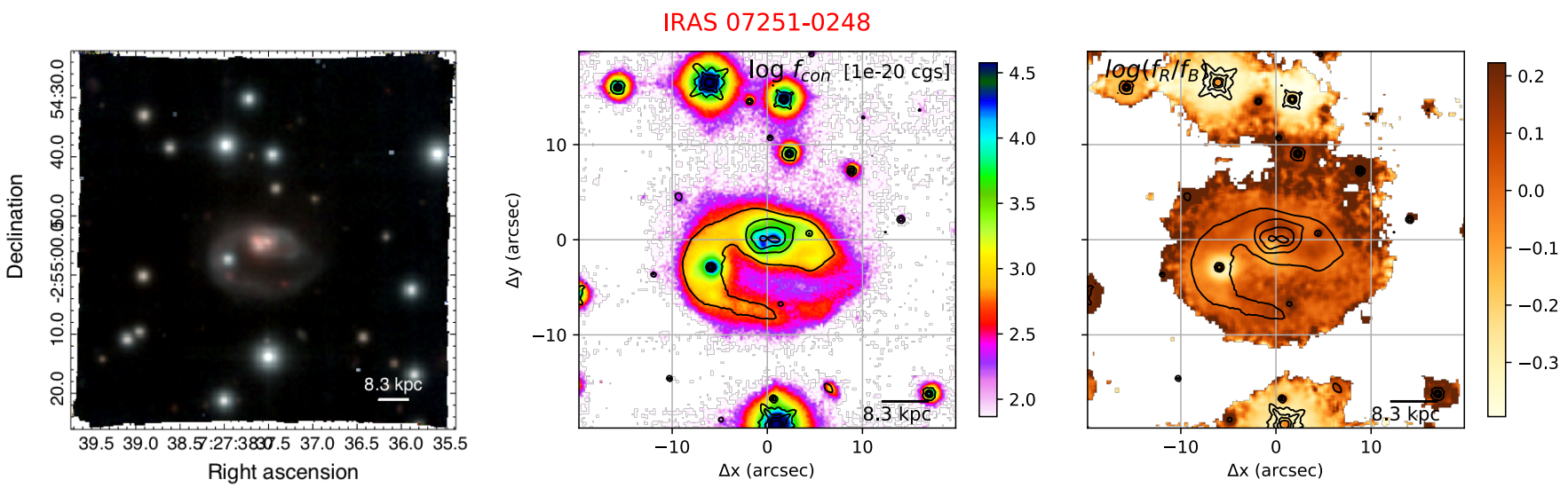

Fig. C.17. IRAS 07251-0248 images from MUSE observations with TOT $=2.55$ hr. Left: Colour-composite optical image showing [O III] (green, from the wavelength range $4995-5020 \AA$ rest-frame), $\mathrm{H} \alpha$ (red, $6554-6575 \AA$ ), and stellar continuum (blue, $4430-4540 \AA$ ). Centre: Red

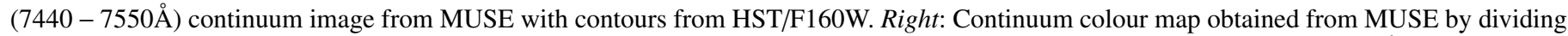
the red continuum image (central panel) from a blue image obtained by collapsing the stellar emission in the range 4430 - $4540 \AA$; contours from HST/F160W.
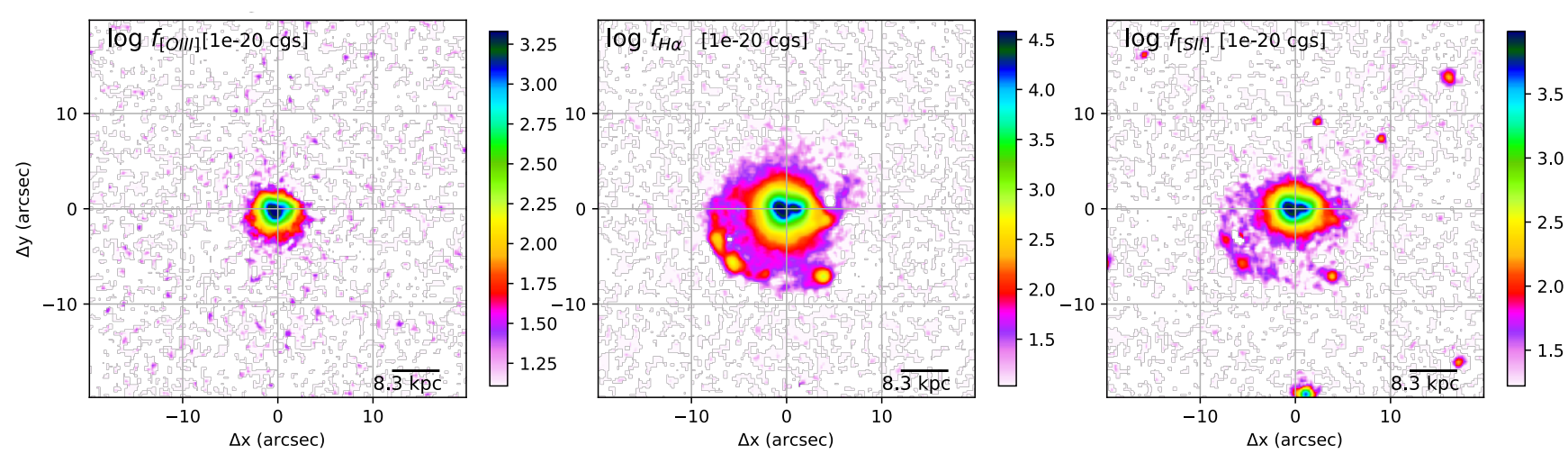

Fig. C.18. IRAS 07251-0248 emission line images from MUSE observations. [O III] (left, from the wavelength range 4995 - 5020 ̊ rest-frame), $\mathrm{H} \alpha$ (centre, $6554-6575 \AA$ ), and [S II] (right, $6704-6746 \AA$ ) images have been obtained by subtracting continuum emission using the adjacent regions at shorter and longer wavelengths with respect to the emission line systemics.
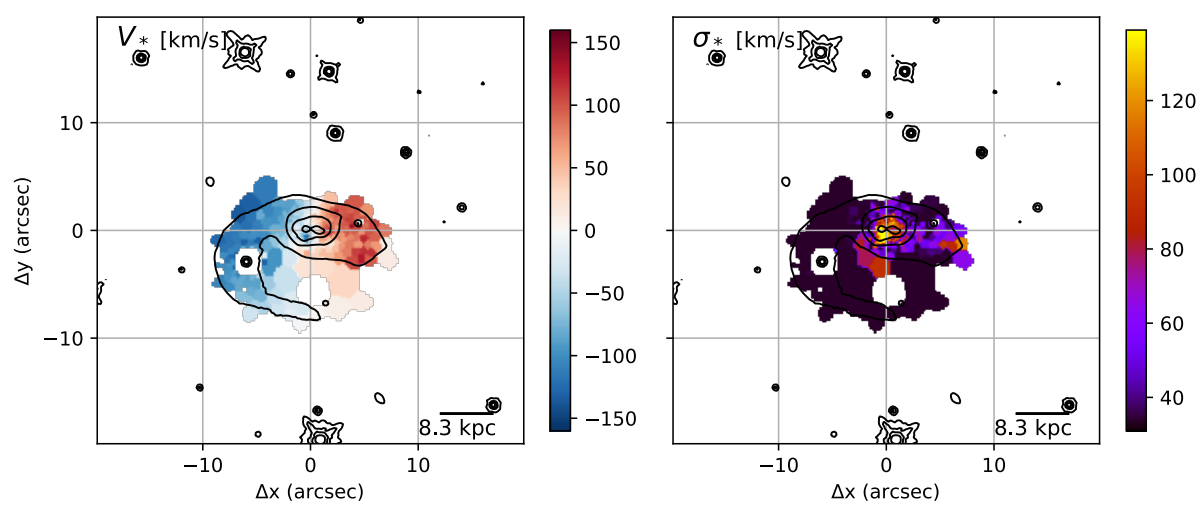

Fig. C.19. IRAS 07251-0248 stellar kinematic maps from the pPXF analysis with contours from HST/F160W. The left panel shows the stellar velocity $V_{*}$, and the right panel represents the velocity dispersion $\sigma_{*}$. 


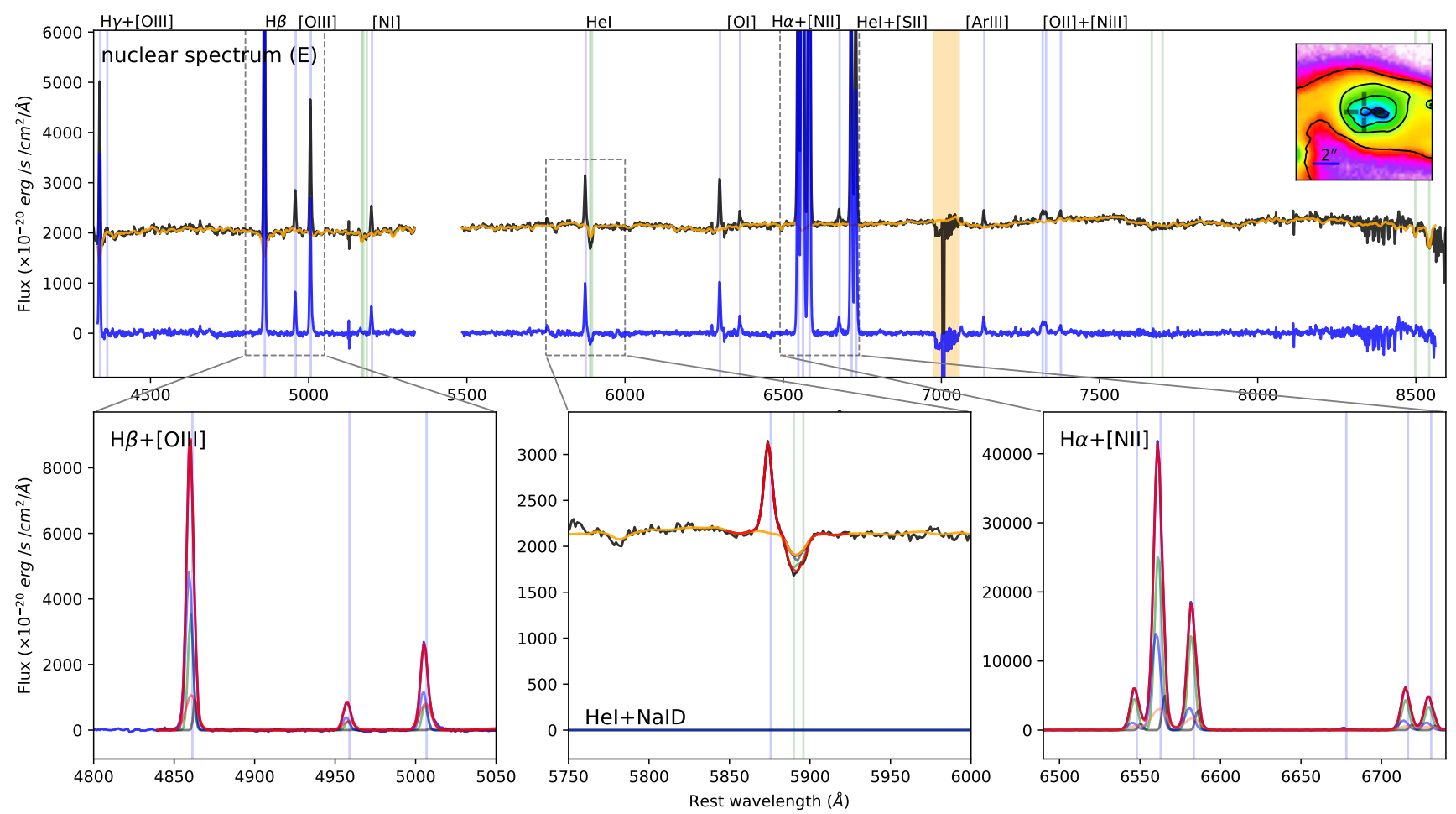

Fig. C.20. IRAS 07251-0248 E nuclear spectrum extracted from a circular aperture with $r<0.4^{\prime \prime}$, with the corresponding pPXF (top panel) and multi-component (bottom insets) best-fit models. See Fig. C.4 for details.

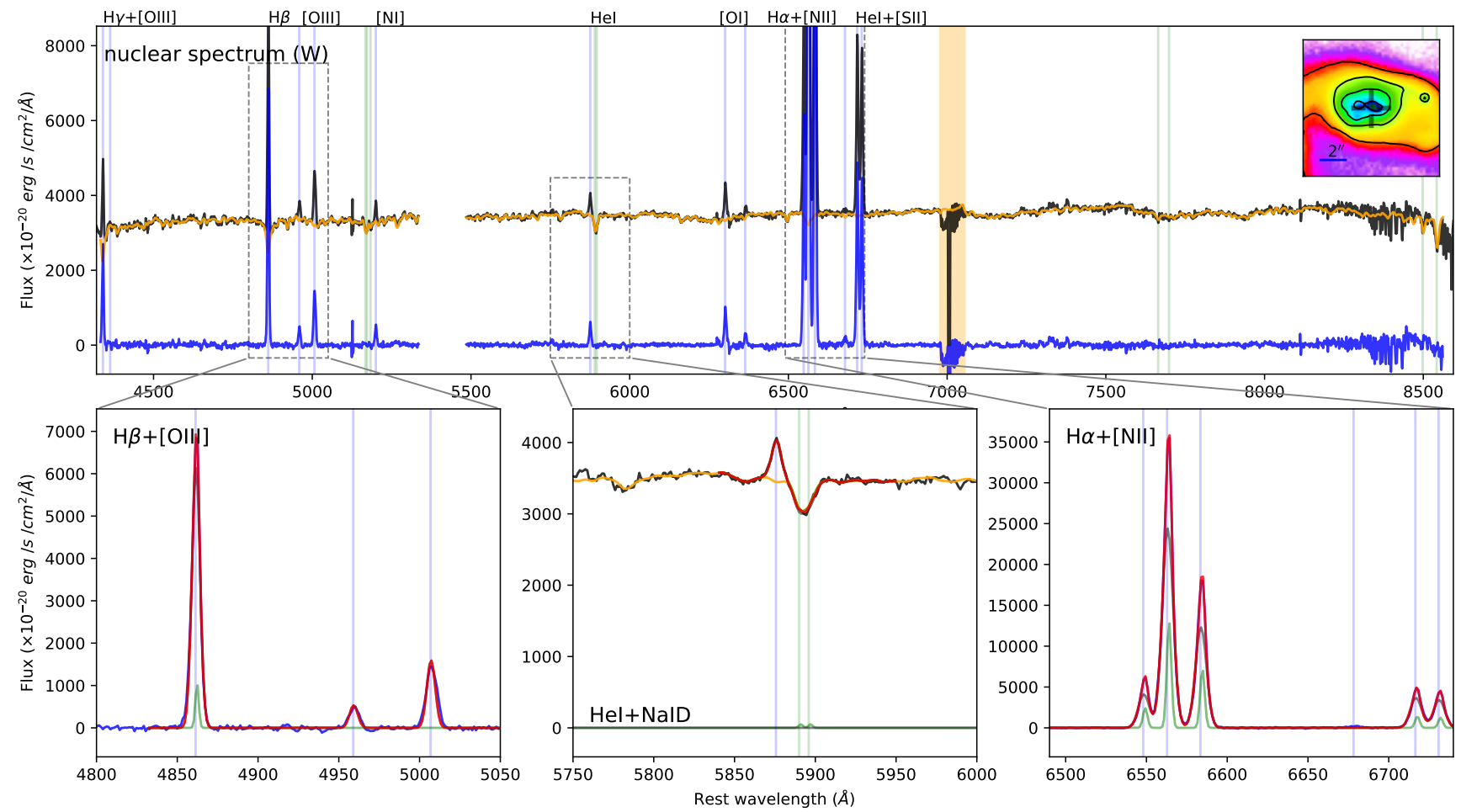

Fig. C.21. IRAS 07251-0248 W nuclear spectrum extracted from a circular aperture with $r<0.4^{\prime \prime}$, with the corresponding pPXF (top panel) and multi-component (bottom insets) best-fit models. See Fig. C.4 for details. 

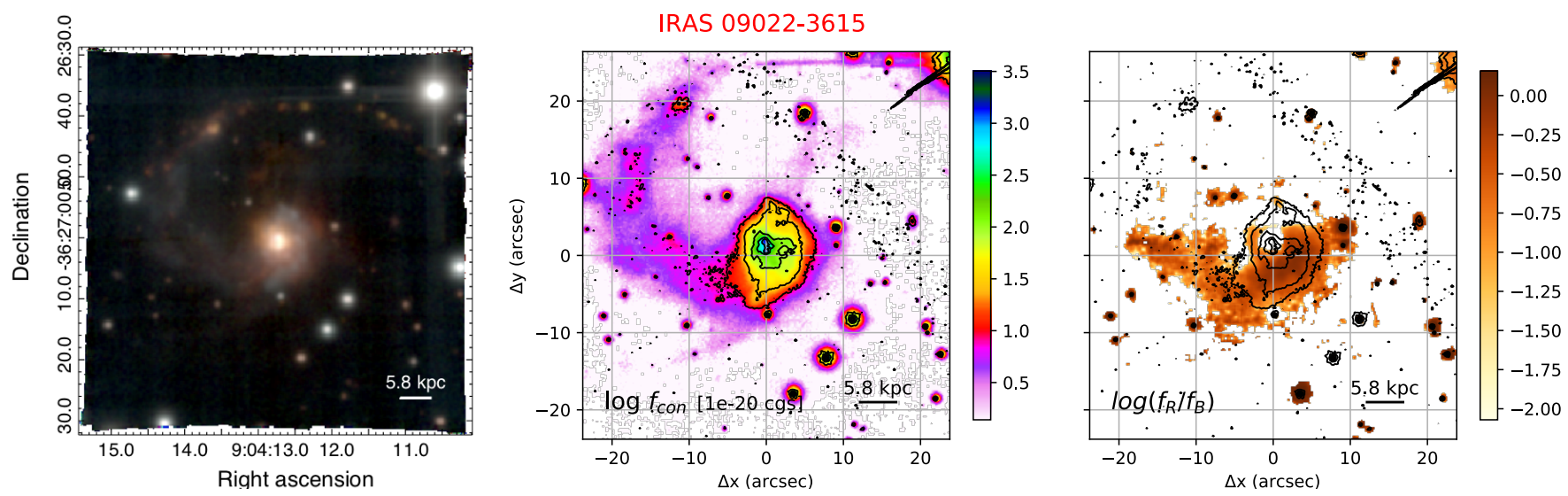

Fig. C.22. IRAS 09022-3615 images from MUSE observations with TOT $=2.04 \mathrm{hr}$. Left: Colour-composite optical image showing [O III] (green, from the wavelength range $4995-5020 \AA$ rest-frame), $\mathrm{H} \alpha$ (red, $6552-6574 \AA$ ), and stellar continuum (blue, $4550-4710 \AA$ ). Centre: Red $(7600-7760 \AA)$ stellar continuum image from MUSE with contours from HST/F814W. Right: Stellar continuum colour map obtained from MUSE by dividing the red continuum image (central panel) by a blue image obtained by collapsing the stellar emission in the range $4550-4710 \AA$; contours from HST/F814W.
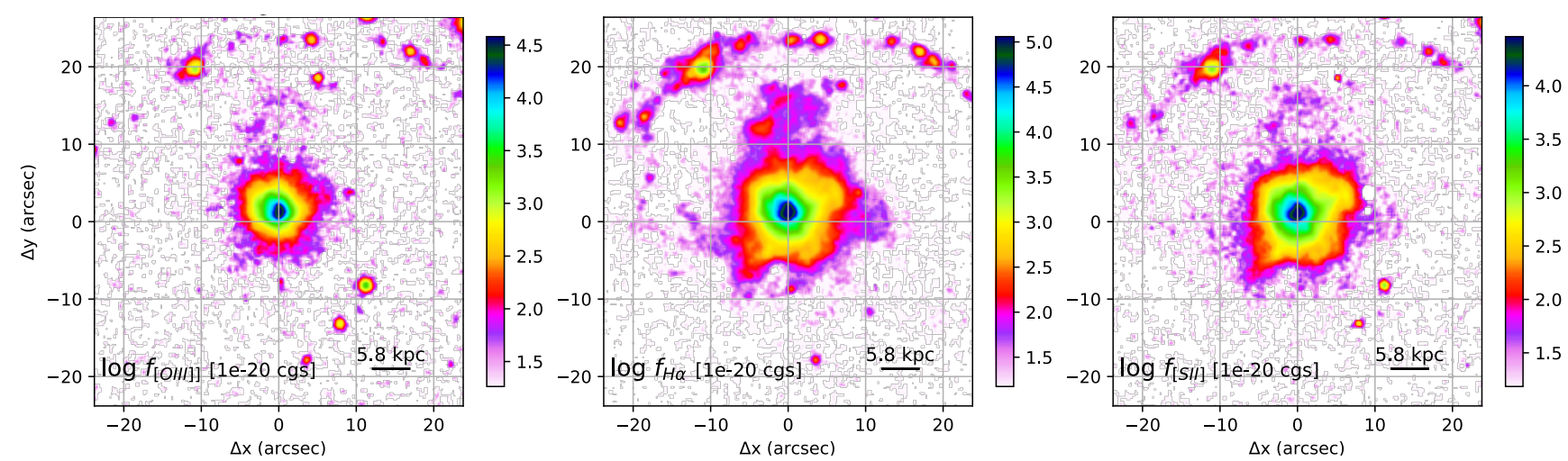

Fig. C.23. IRAS 09022-3615 emission line images from MUSE observations. [O III] (left, from the wavelength range $4995-5020 \AA$ rest-frame),

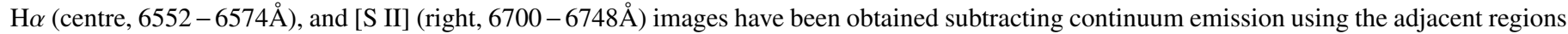
at shorter and longer wavelengths with respect to the emission line systemics.
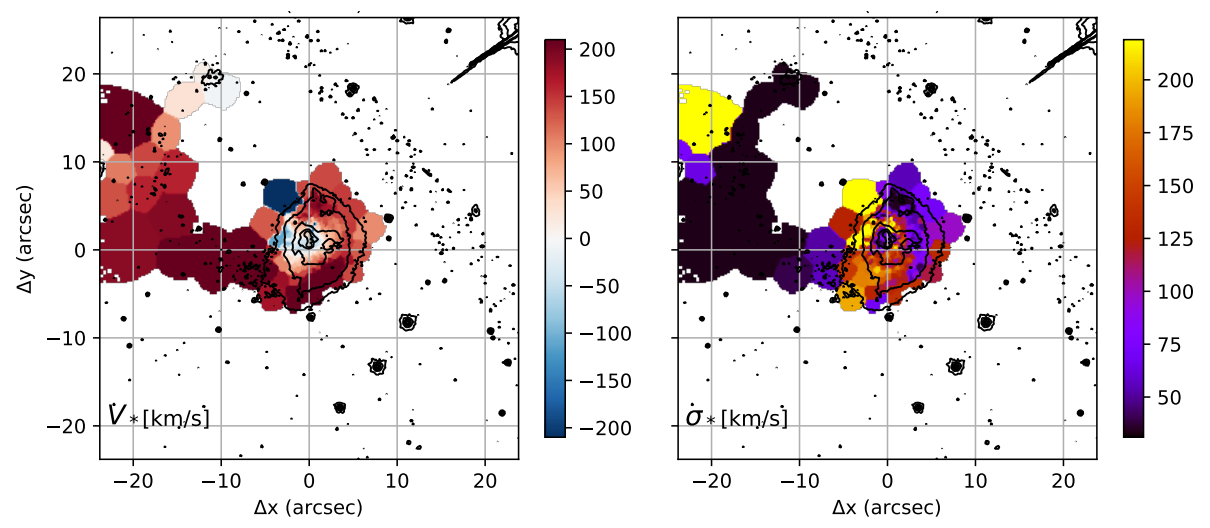

Fig. C.24. IRAS 09022-3615 stellar kinematic maps from the pPXF analysis with contours from HST/F814W. The left panel shows the stellar velocity $V_{*}$, and the right panel represents the velocity dispersion $\sigma_{*}$. 


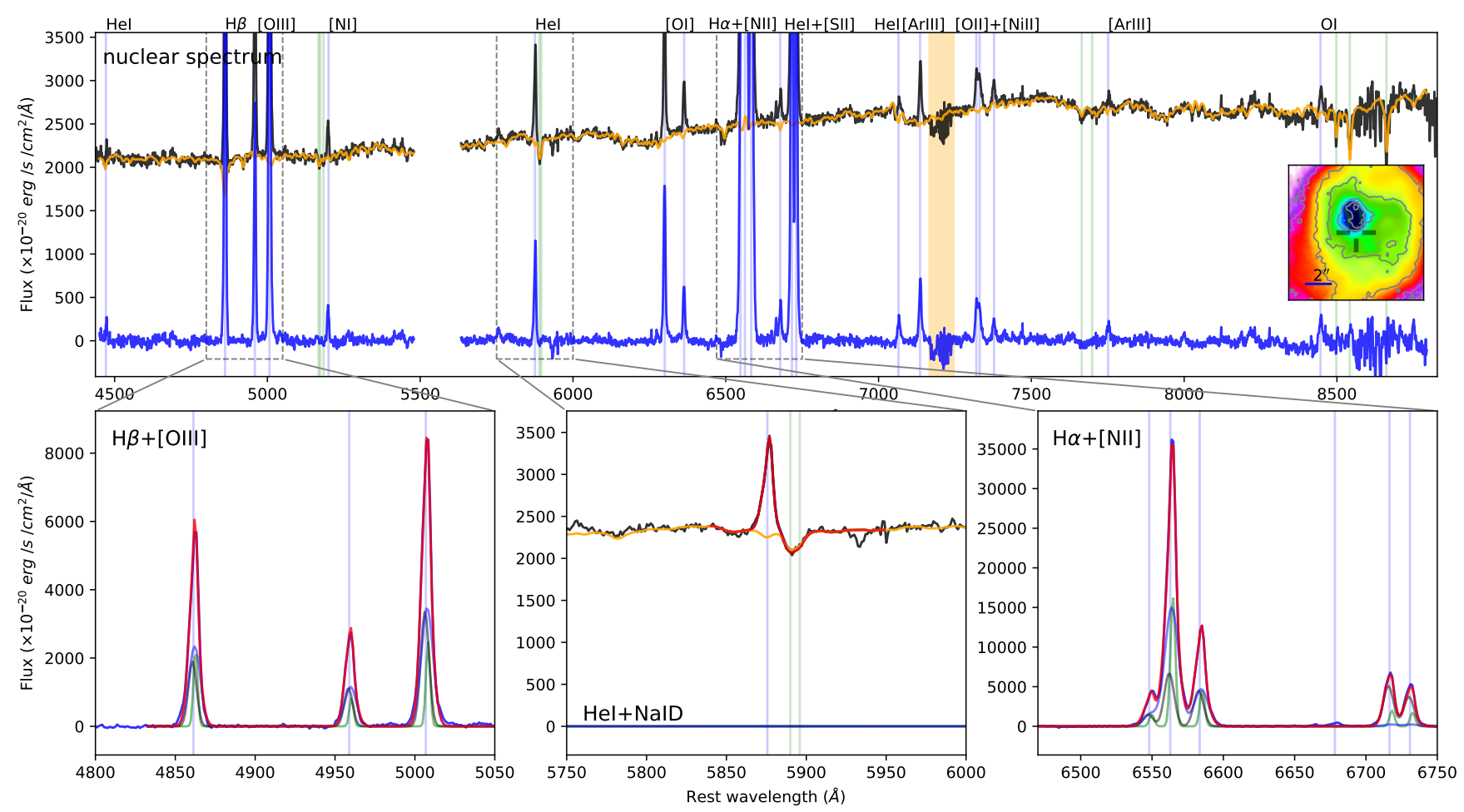

Fig. C.25. IRAS 09022 nuclear spectrum extracted from a circular aperture with $r<0.4^{\prime \prime}$, with the corresponding pPXF (top panel) and multicomponent (bottom insets) best-fit models. See Fig. C.4 for details. 

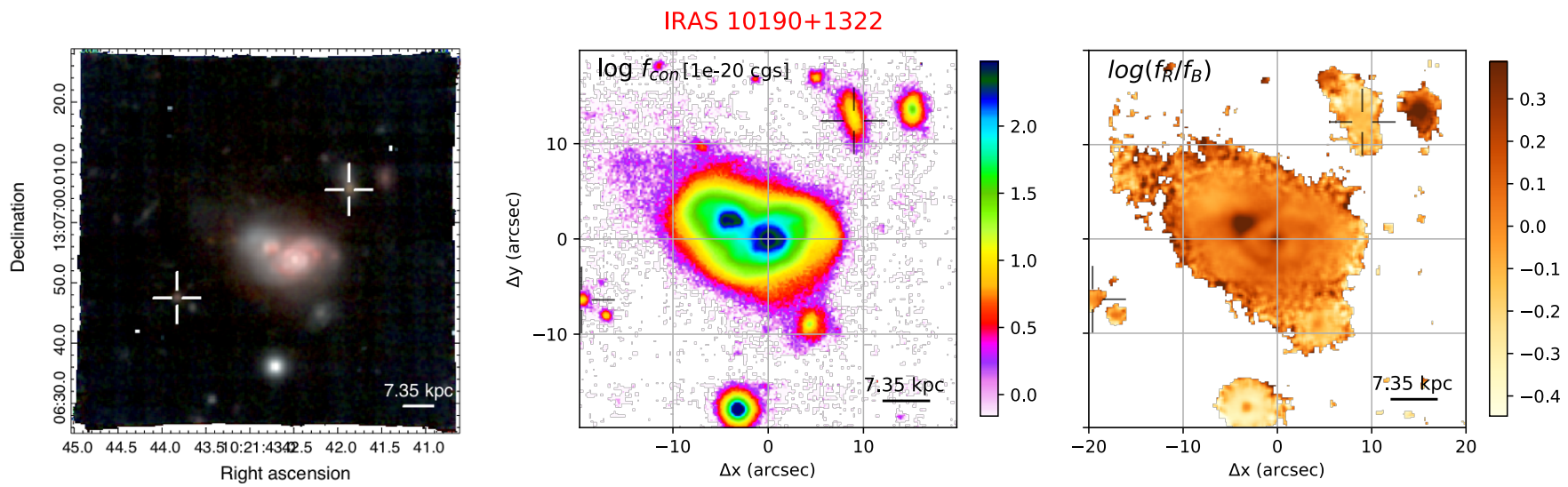

Fig. C.26. IRAS 10190+1322 images from MUSE observations with TOT $=2.04 \mathrm{hr}$. Left: Colour-composite optical image showing [O III] (green, from the wavelength range 4988 - 5022 ̊ rest-frame), H $\alpha$ (red, $6556-6572 \AA$ ), and stellar continuum (blue, $4460-4570 \AA$ ). Centre: Red

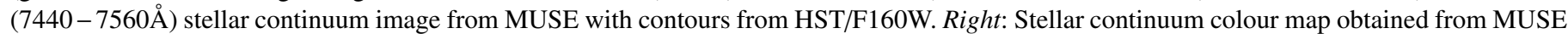
by dividing the red continuum image (central panel) by a blue image obtained by collapsing the stellar emission in the range $4460-4570 \AA$. In all panels, we display the two companion galaxies of IRAS 10190+1322 with cross markers.
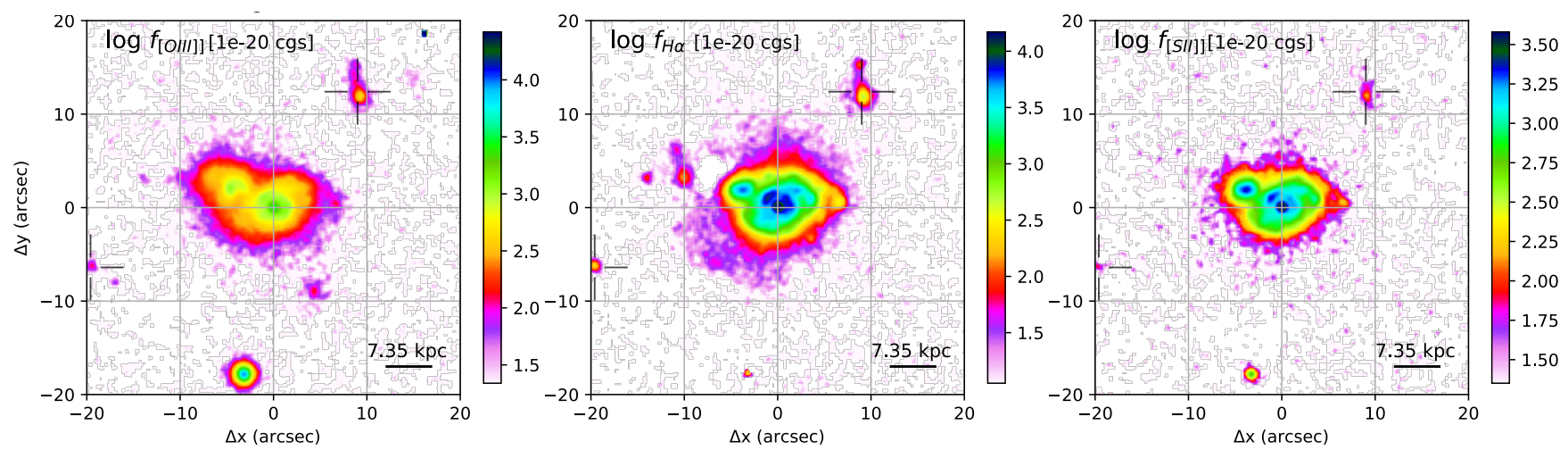

Fig. C.27. IRAS 10190+1322 emission line images from MUSE observations. [O III] (left, from the wavelength range $4988-5022 \AA ̊$ rest-frame), $\mathrm{H} \alpha$ (centre, $6556-6572 \AA$ ), and [S II] (right, $6704-6747 \AA$ ) images have been obtained by subtracting continuum emission using the adjacent regions at shorter and longer wavelengths with respect to the emission line systemics.
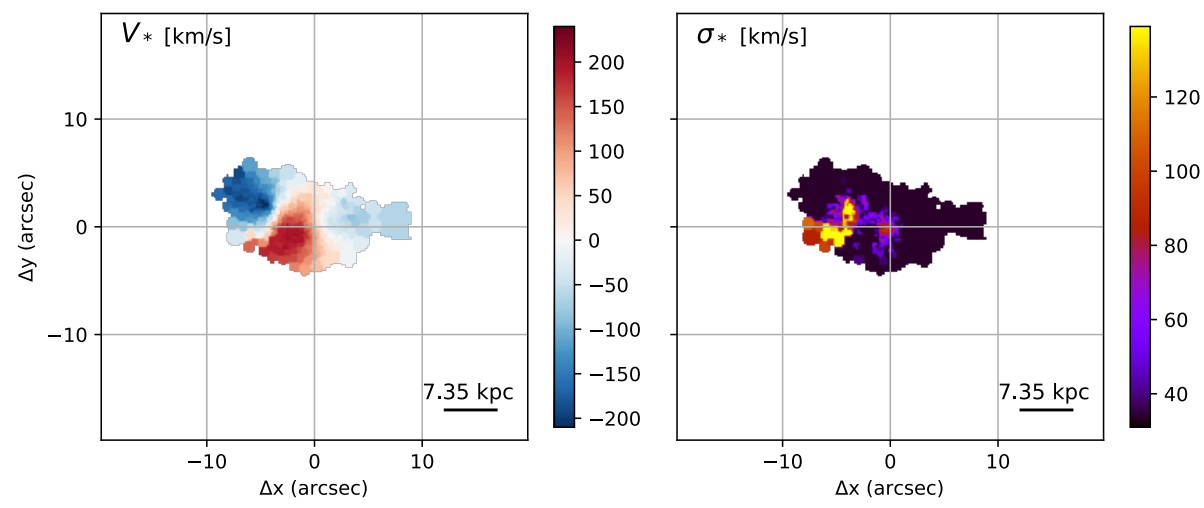

Fig. C.28. IRAS 10190+1322 stellar kinematic maps from the pPXF analysis. The left panel shows the stellar velocity $V_{*}$, and the right panel represents the velocity dispersion $\sigma_{*}$. 


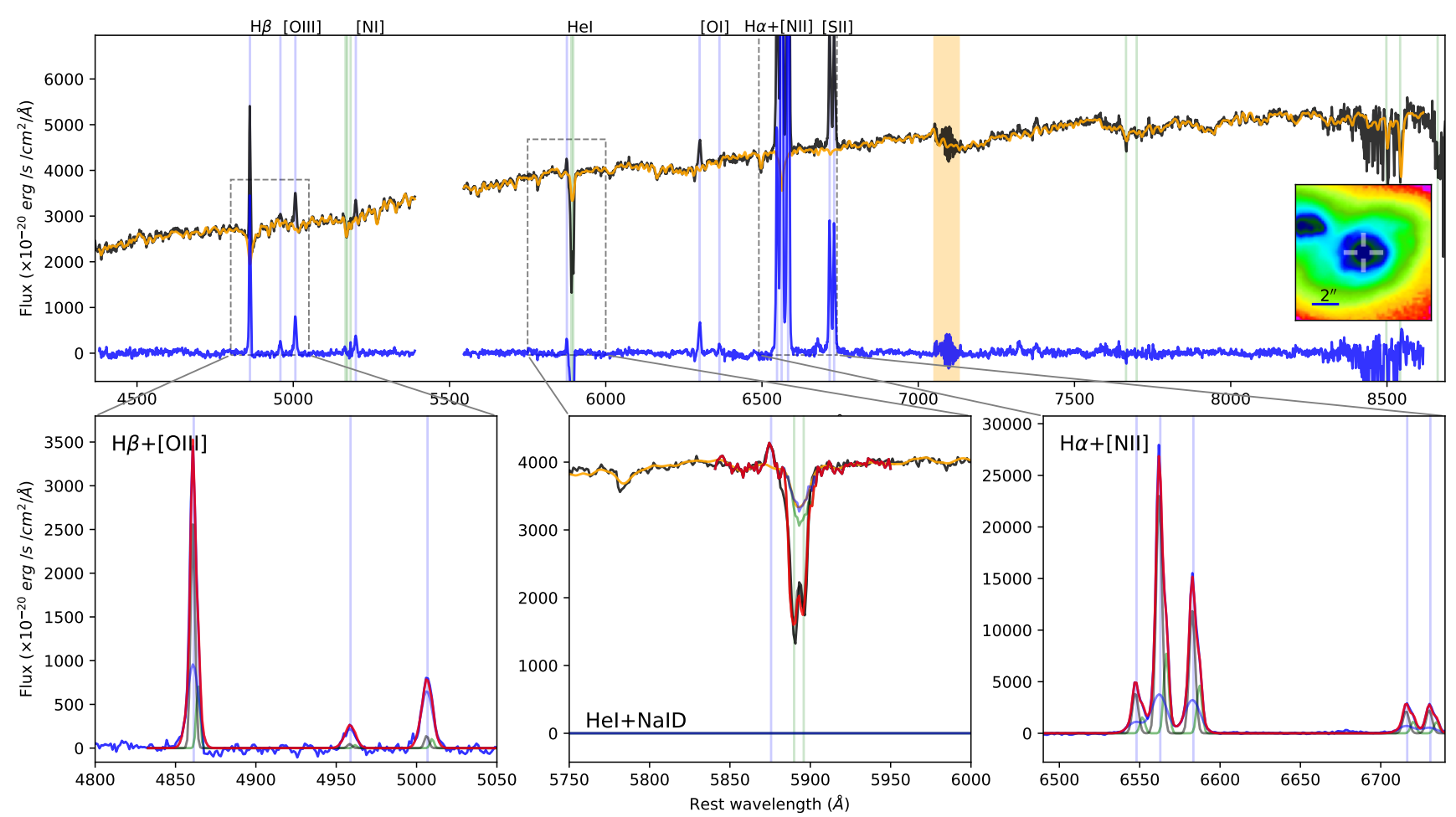

Fig. C.29. IRAS 10190+1322 W nuclear spectrum extracted from a circular aperture with $r<0.4^{\prime \prime}$, with the corresponding pPXF (top panel) and multi-component (bottom insets) best-fit models. See Fig. C.4 for details.

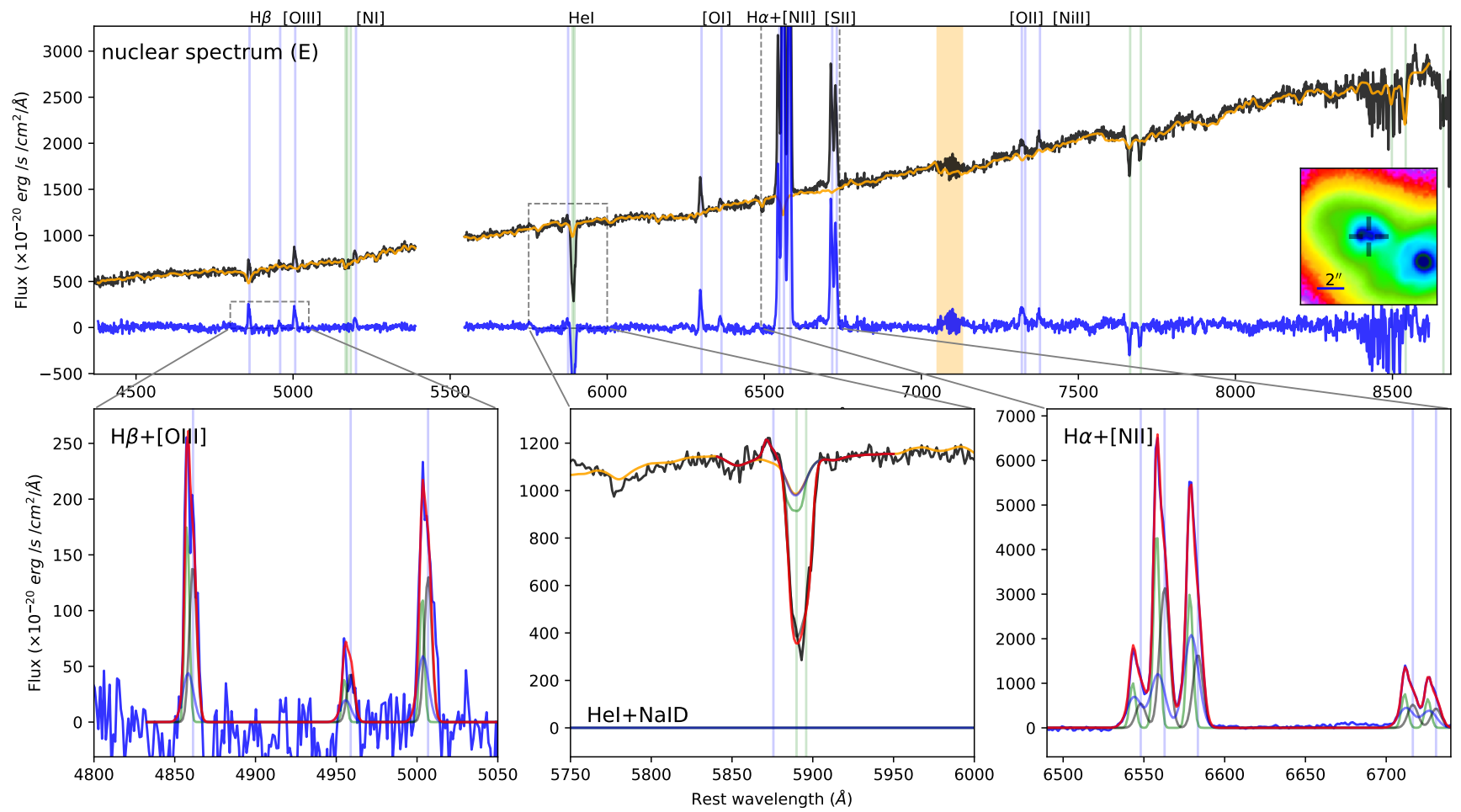

Fig. C.30. IRAS 10190+1322 E nuclear spectrum extracted from a circular aperture with $r<0.4^{\prime \prime}$, with the corresponding pPXF (top panel) and multi-component (bottom insets) best-fit models. See Fig. C.4 for details. 

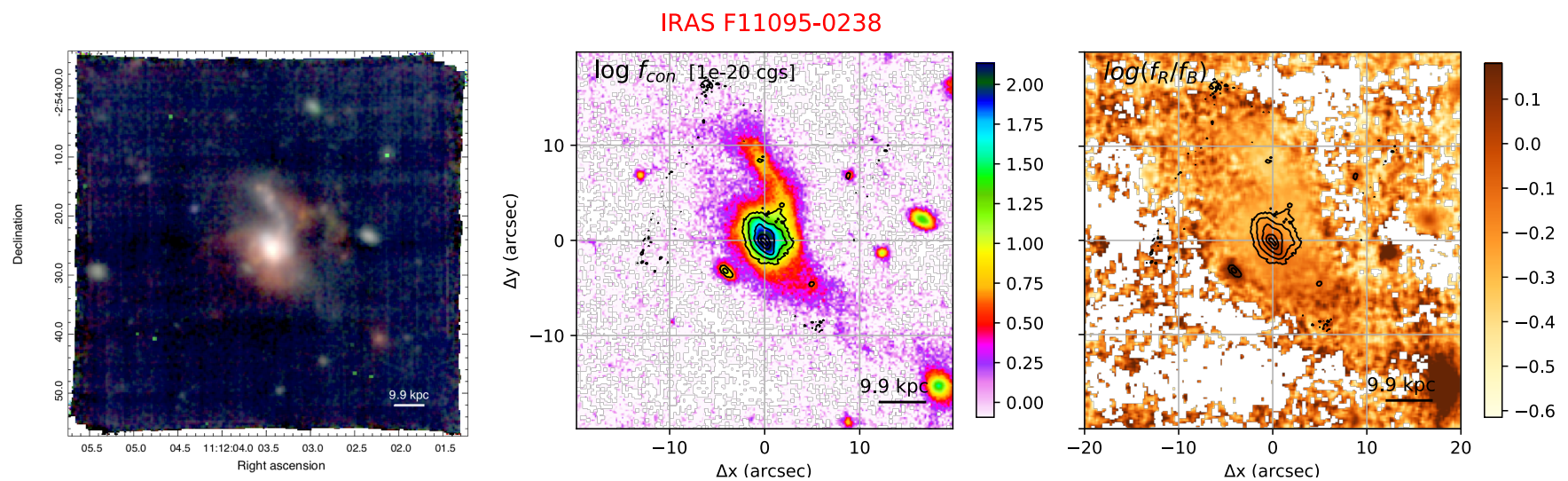

Fig. C.31. IRAS F11095-0238 images from MUSE observations with TOT = $2.72 \mathrm{hr}$. Left: Colour-composite optical image showing [O III] (green, from the wavelength range $4975-5032 \AA$ rest-frame), $\mathrm{H} \alpha$ (red, $6549-6576 \AA$ ), and continuum (blue, $4410-4490 \AA$ ). Centre: Red $(7690-7760 \AA)$ continuum image from MUSE, with contours from HST/F160W. Right: Continuum colour map obtained from MUSE by dividing the red continuum image (central panel) by a blue image obtained by collapsing the stellar emission in the range $4410-4490 \AA$; contours from HST/F160W.
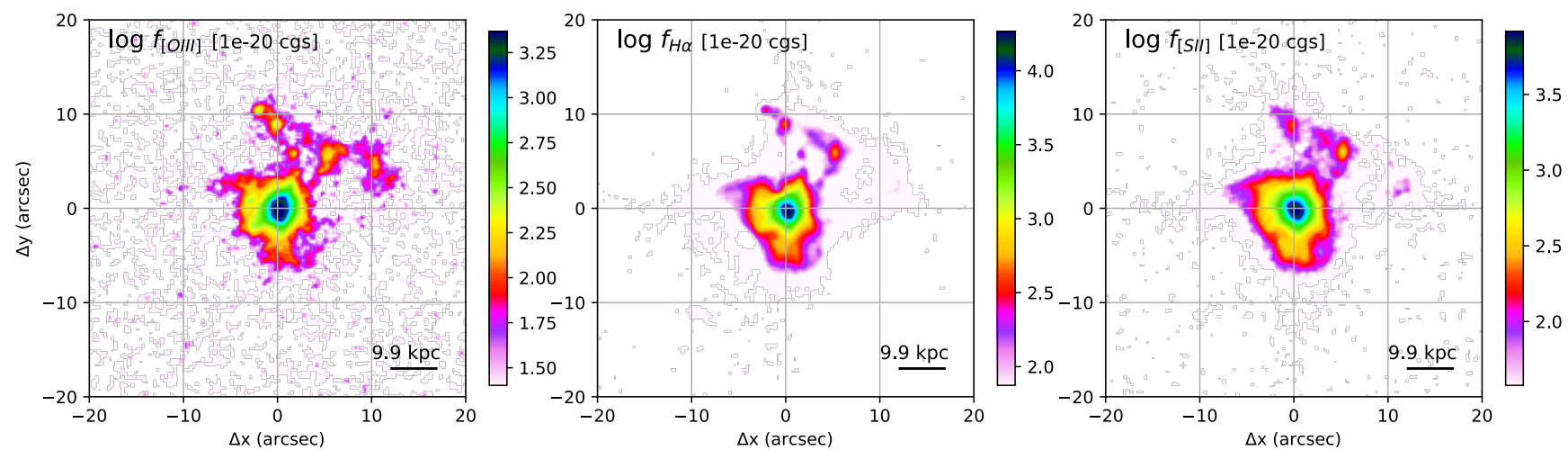

Fig. C.32. IRAS F11095-0238 emission line images from MUSE observations. [O III] (left, from the wavelength range 4975 - 5032Å rest-frame), $\mathrm{H} \alpha$ (centre, $6549-6576 \AA$ ), and [S II] (right, $6695-6749 \AA$ ) images have been obtained by subtracting continuum emission using the adjacent regions at shorter and longer wavelengths with respect to the emission line systemics.
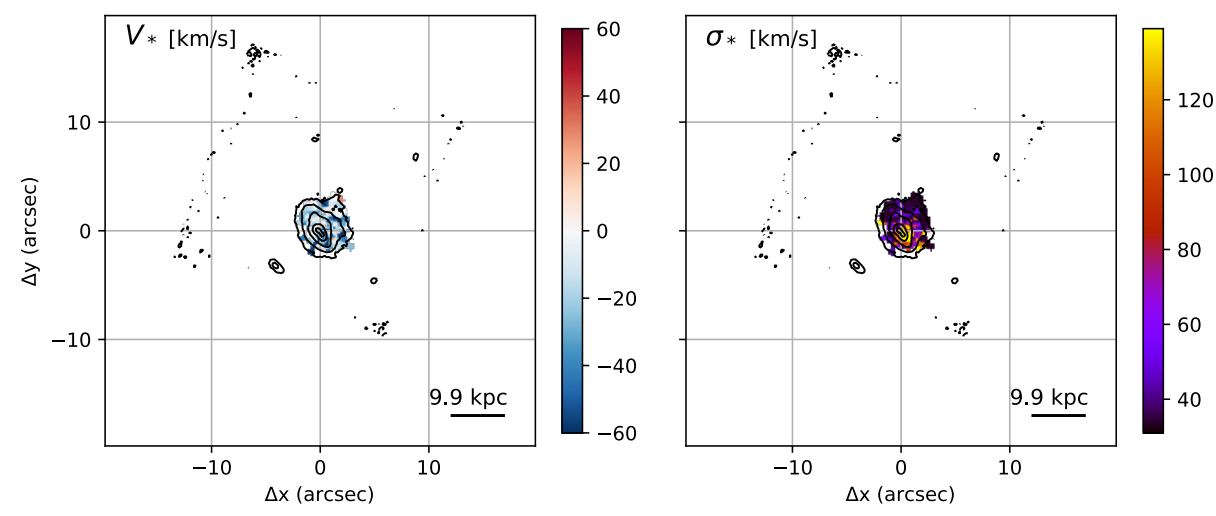

Fig. C.33. IRAS F11095-0238 stellar kinematic maps from the pPXF analysis with contours from HST/F160W. The left panel shows the stellar velocity $V_{*}$, and the right panel represents the velocity dispersion $\sigma_{*}$. 


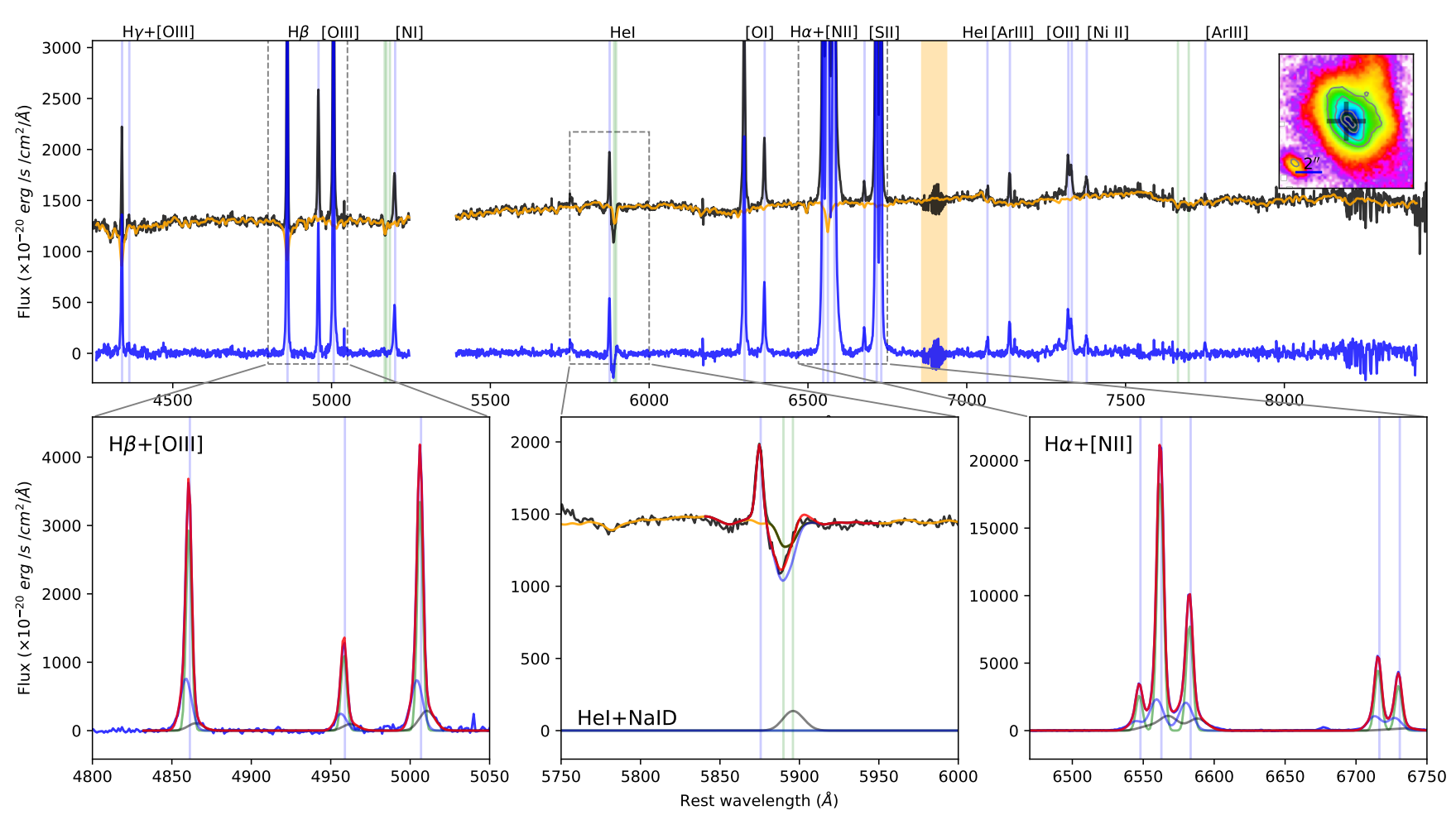

Fig. C.34. IRAS F11095-0238 NE nuclear spectrum extracted from a circular aperture with $r<0.4^{\prime \prime}$, with the corresponding pPXF (top panel) and multi-component (bottom insets) best-fit models. See Fig. C.4 for details.

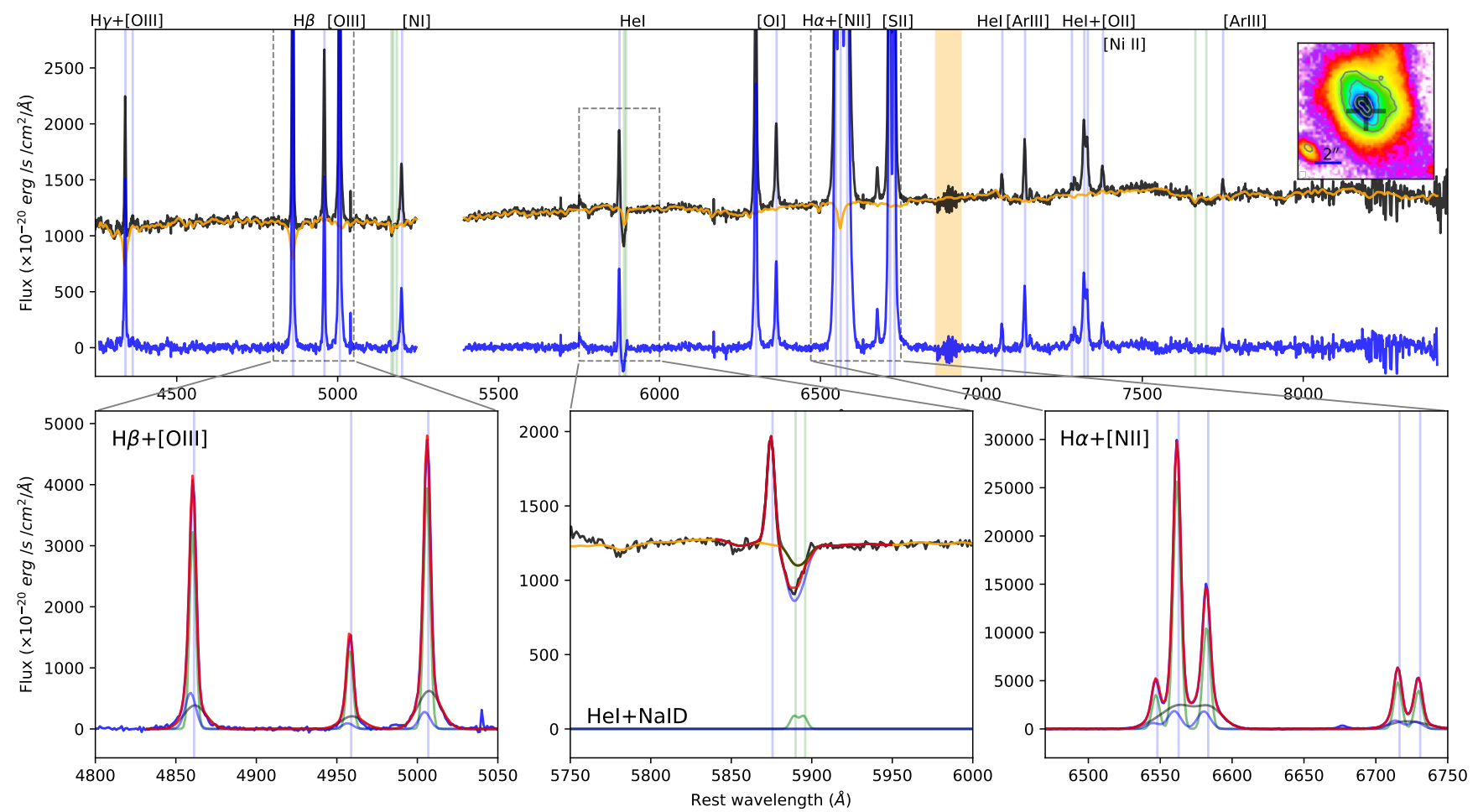

Fig. C.35. IRAS F11095-0238 SW nuclear spectrum extracted from a circular aperture with $r<0.4^{\prime \prime}$, with the corresponding pPXF (top panel) and multi-component (bottom insets) best-fit models. See Fig. C.4 for details. 

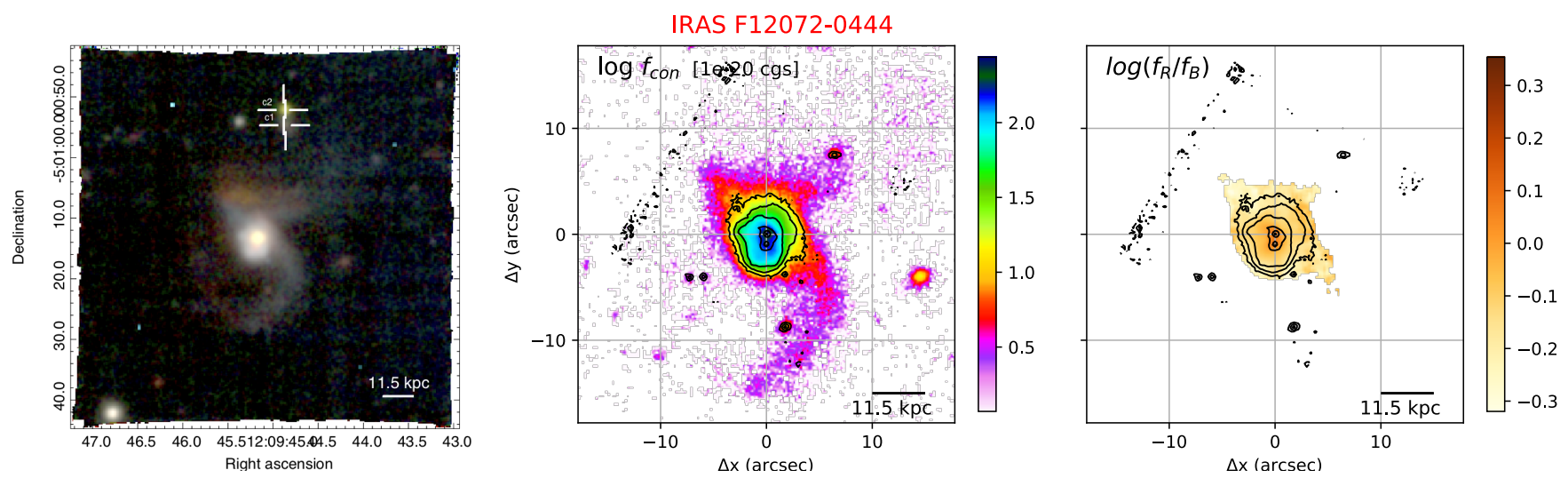

Fig. C.36. IRAS F12072-0444 images from MUSE observations with TOT $=0.68 \mathrm{hr}$. Left: Colour-composite optical image showing [O III]

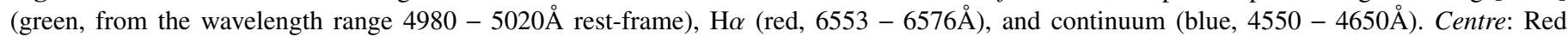
(7540 - 7610 $)$ continuum image from MUSE, with contours from HST/F160W. Right: Continuum colour map obtained from MUSE by dividing the red continuum image (central panel) by a blue image obtained by collapsing the stellar emission in the range $4550-4650 \AA$; contours from HST/F160W. In the first panel, we display the IRAS F12072-0444 companion galaxies with crosses.
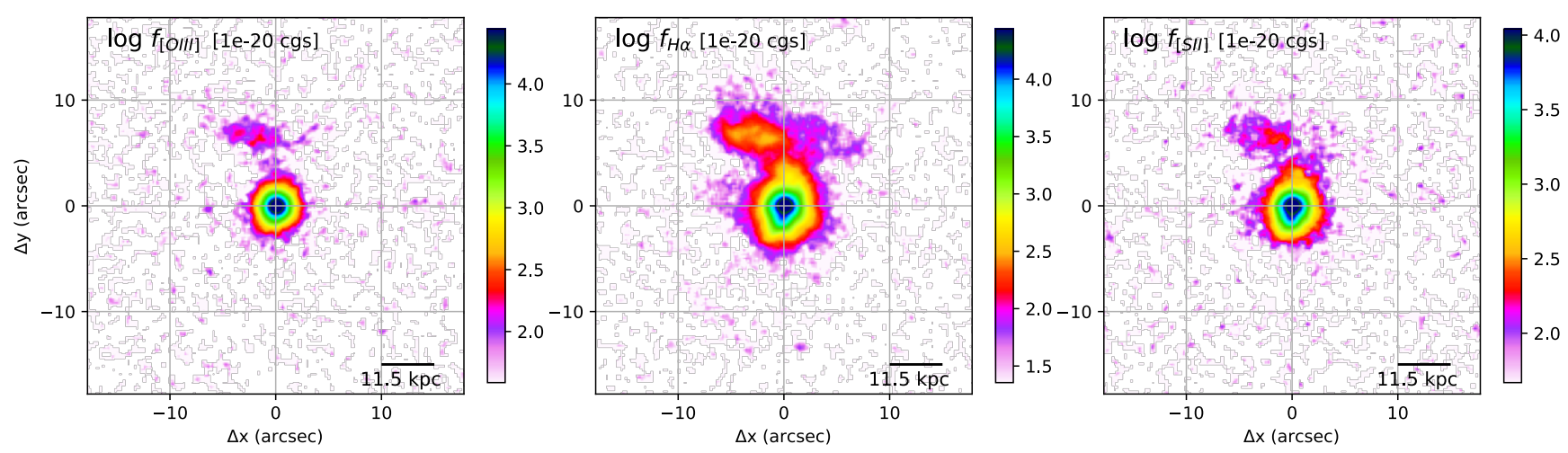

Fig. C.37. IRAS F12072-0444 emission line images from MUSE observations. [O III] (left, from the wavelength range 4980 - 5020 ̊ rest-frame), $\mathrm{H} \alpha$ (centre, $6553-6576 \AA$ ), and [S II] (right, $6695-6747 \AA$ ) images have been obtained by subtracting continuum emission using the adjacent regions at shorter and longer wavelengths with respect to the emission line systemics.
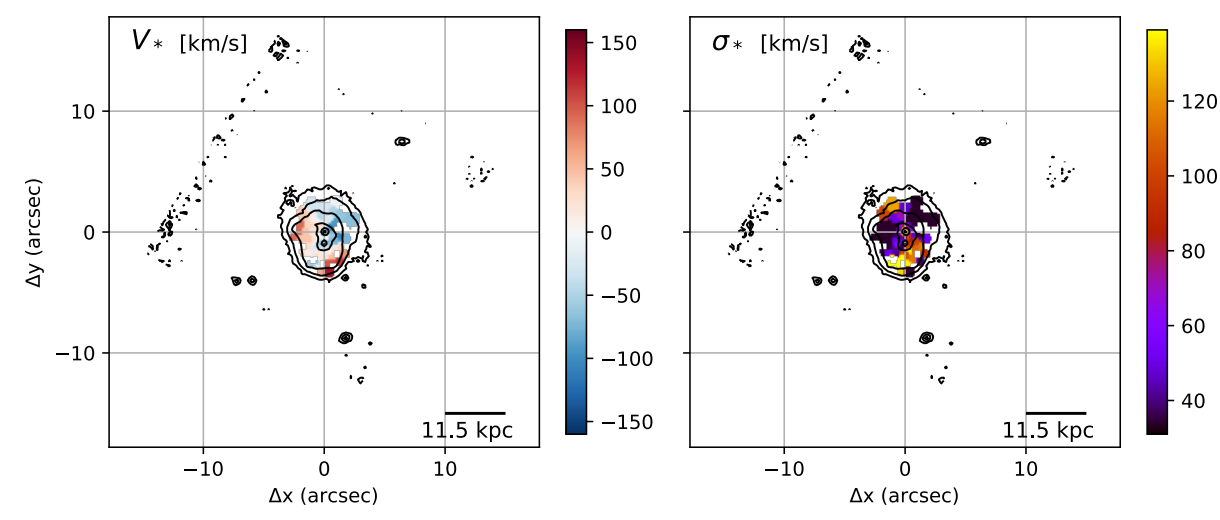

Fig. C.38. IRAS F12072-0444 stellar kinematic maps from the pPXF analysis with contours from HST/F160W. The left panel shows the stellar velocity $V_{*}$, and the right panel represents the velocity dispersion $\sigma_{*}$. 


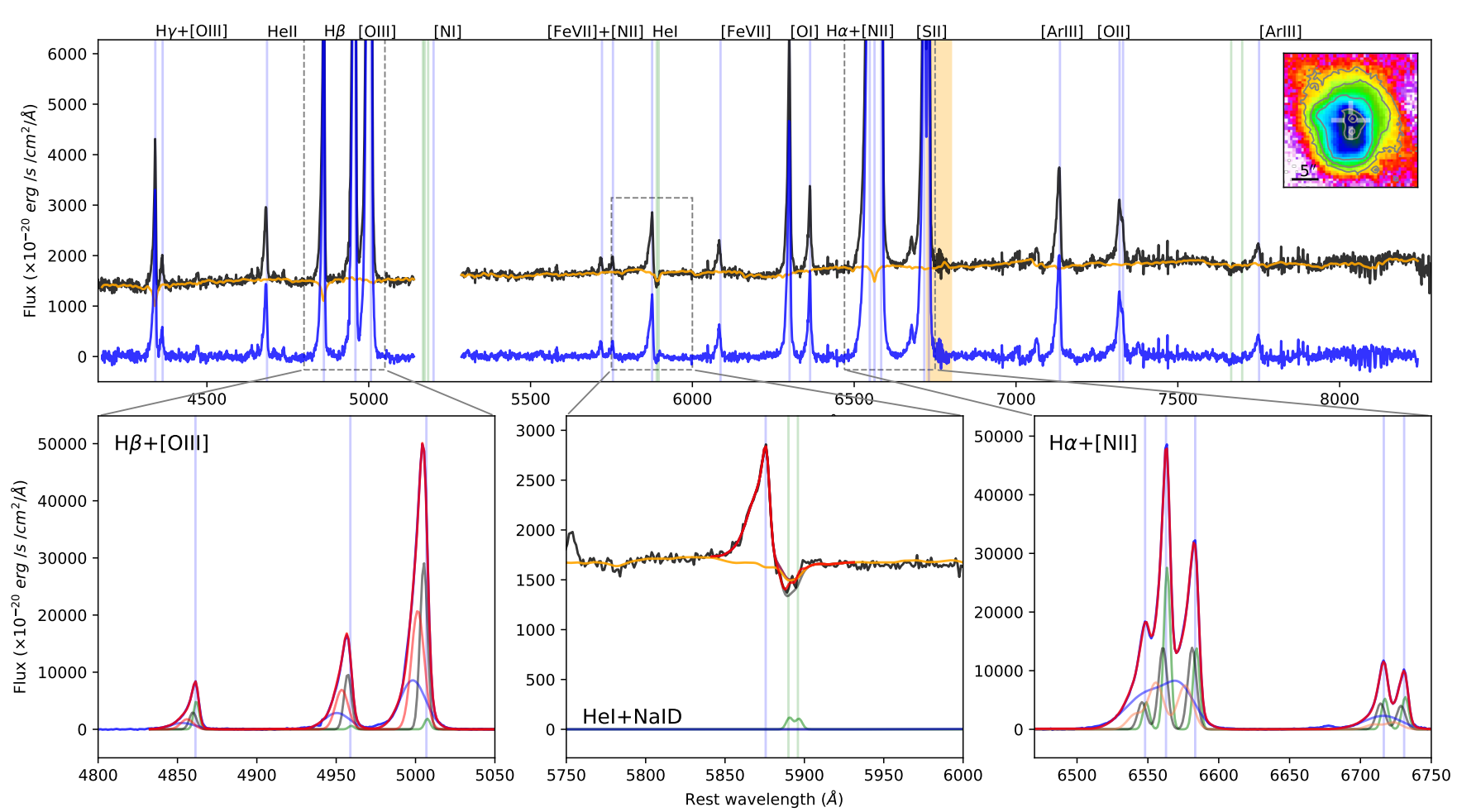

Fig. C.39. IRAS F12072-0444 NE nuclear spectrum extracted from a circular aperture with $r<0.4^{\prime \prime}$, with the corresponding pPXF (top panel) and multi-component (bottom insets) best-fit models. See Fig. C.4 for details.

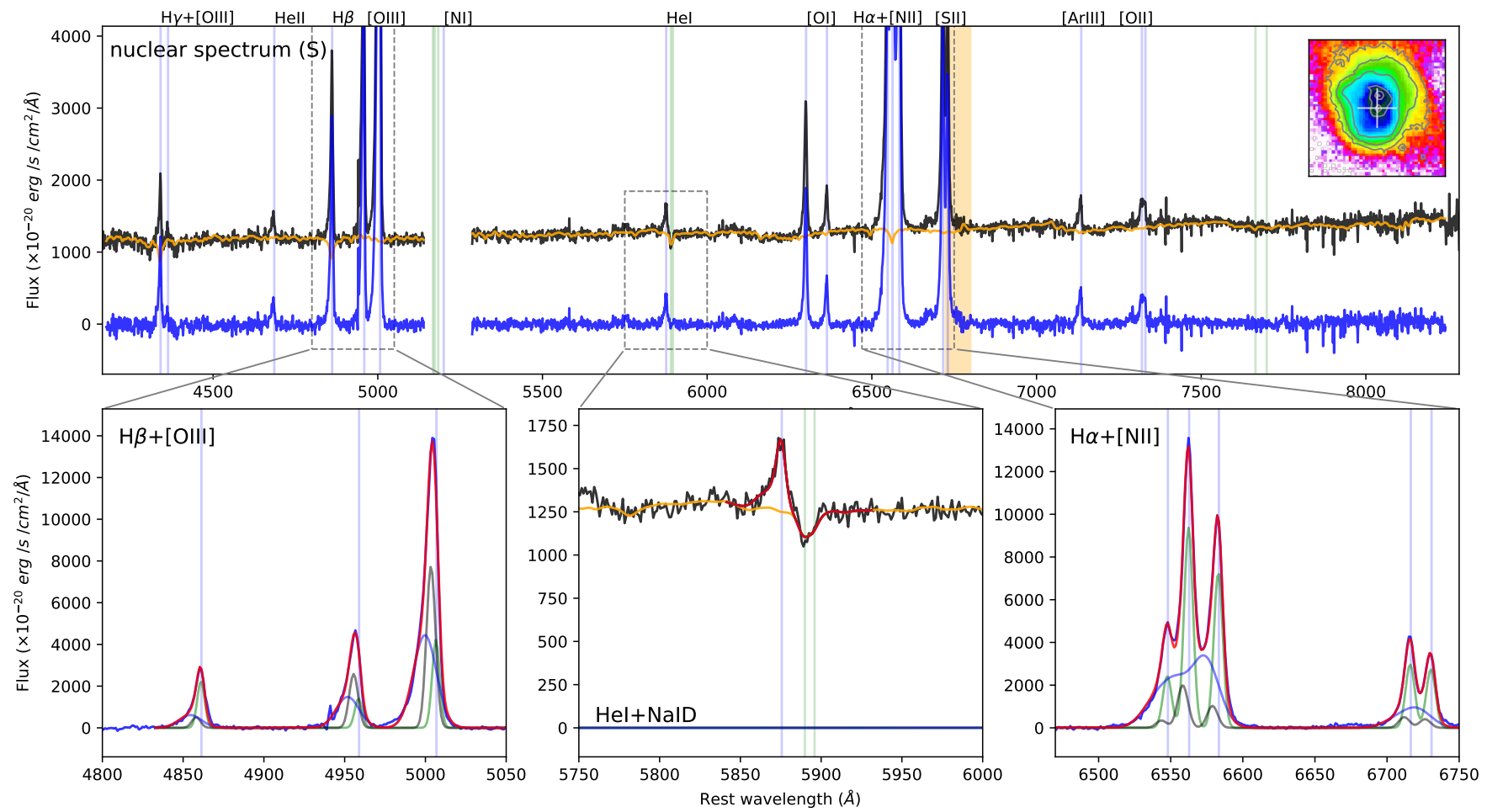

Fig. C.40. IRAS F12072-0444 SW nuclear spectrum extracted from a circular aperture with $r<0.4^{\prime \prime}$, with the corresponding pPXF (top panel) and multi-component (bottom insets) best-fit models. See Fig. C.4 for details. 

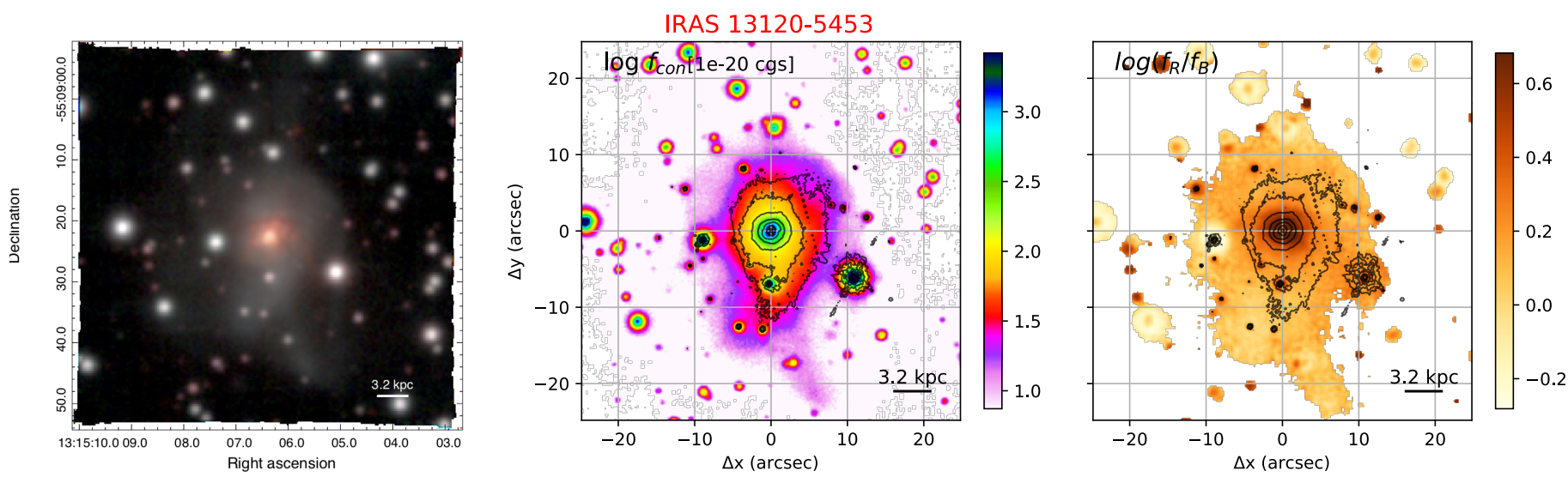

Fig. C.41. IRAS 13120-5453 images from MUSE observations with TOT = $0.52 \mathrm{hr}$. Left: Colour-composite optical image showing [O III] (green, from the wavelength range $4980-5014 \AA$ rest-frame), $\mathrm{H} \alpha$ (red, $6555-6572 \AA$ ), and continuum (blue, $4600-4700 \AA$ ). Centre: red (7500 - 7600 $)$ continuum image from MUSE with contours from HST/F160W. Right: Continuum colour map obtained from MUSE by dividing the red continuum image (central panel) by a blue image obtained by collapsing the stellar emission in the range $4600-4700 \AA$; contours from HST/F160W.
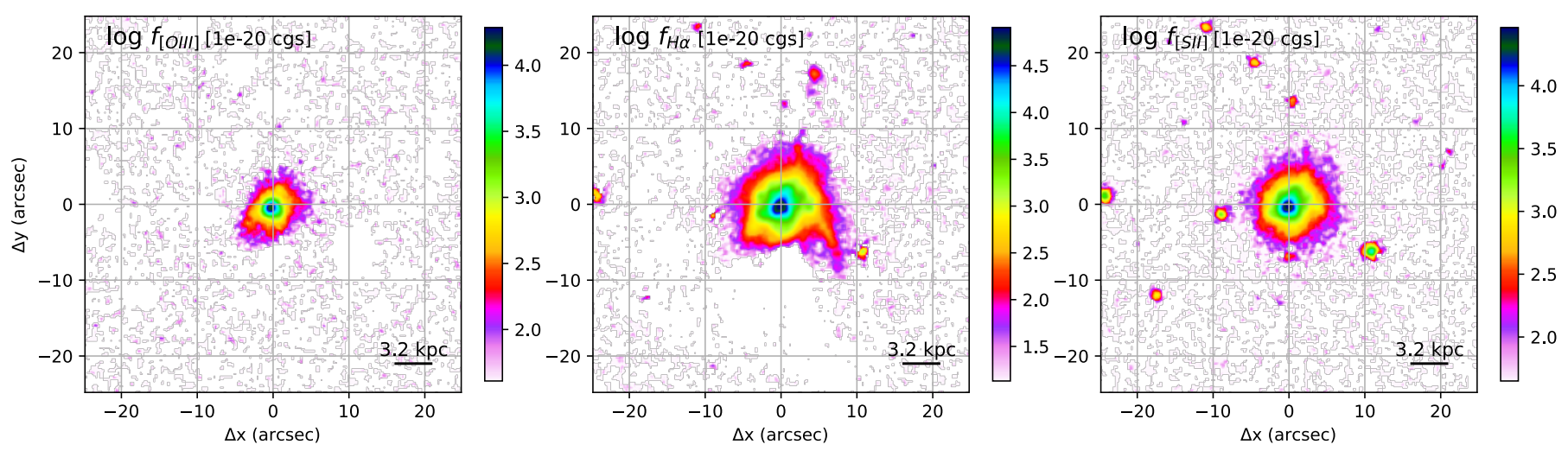

Fig. C.42. IRAS 13120-5453 emission line images from MUSE observations. [O III] (left, from the wavelength range $4980-5014 \AA ̊$ rest-frame), $\mathrm{H} \alpha$ (centre, $6555-6572 \AA$ ), and [S II] (right, $6694-6746 \AA$ ) images have been obtained by subtracting continuum emission using the adjacent regions at shorter and longer wavelengths with respect to the emission line systemics. In all panels, we display the position of the companion galaxy with a cross.
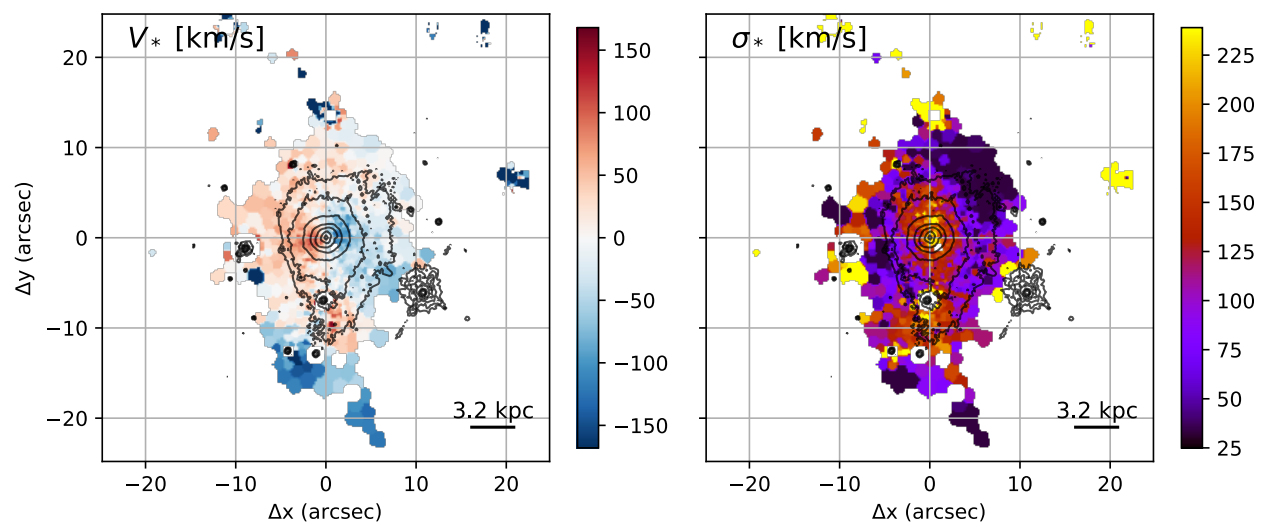

Fig. C.43. IRAS 13120-5453 stellar kinematic maps from the pPXF analysis with contours from HST/F160W. The left panel shows the stellar velocity $V_{*}$, and the right panel represents the velocity dispersion $\sigma_{*}$. The inner $3.5 \mathrm{kpc}$ may indicate a low-amplitude rotational pattern; he outside regions contain more irregular kinematics. 


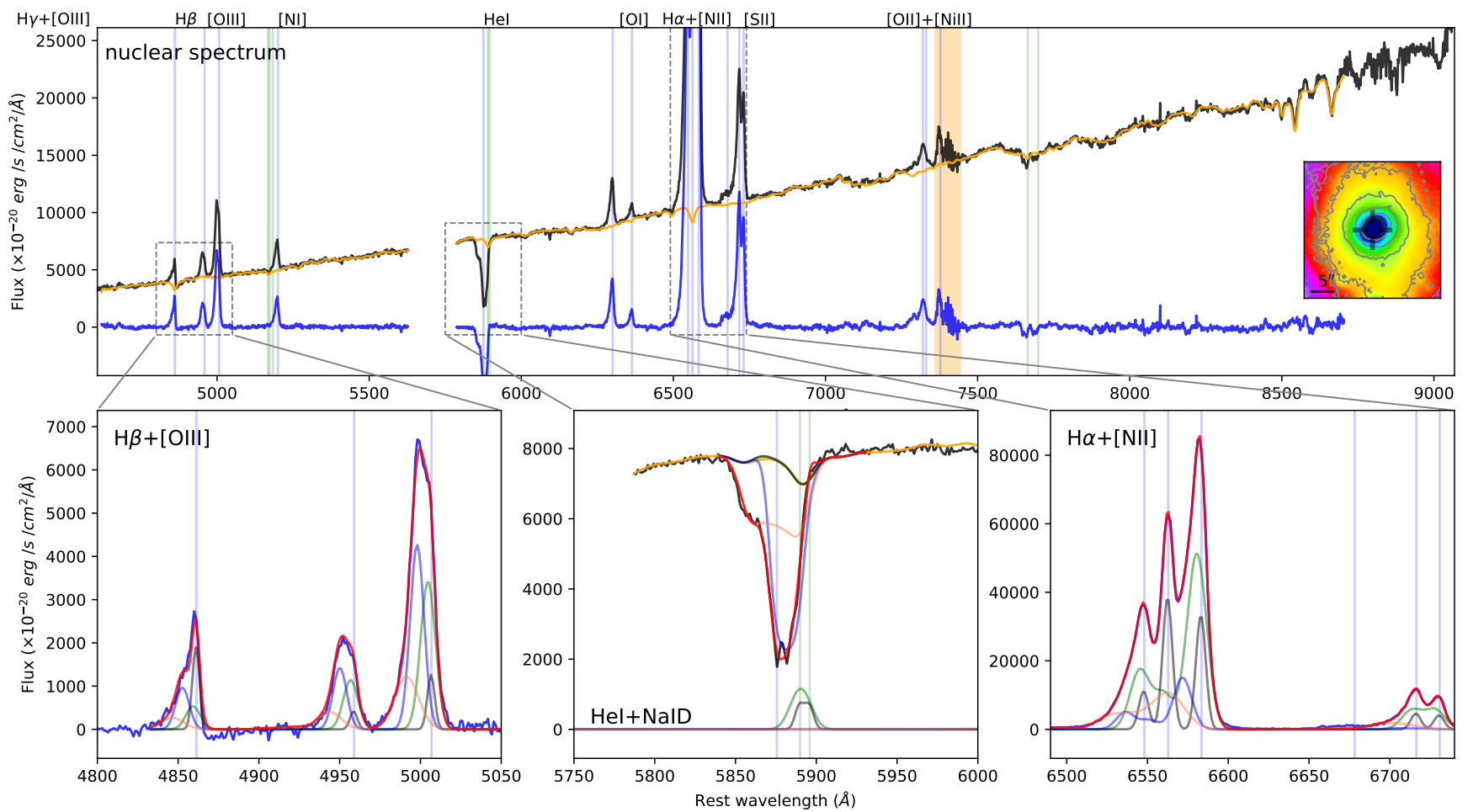

Fig. C.44. IRAS 13120-5453 nuclear spectrum extracted from a circular aperture with $r<0.4^{\prime \prime}$, with the corresponding pPXF (top panel) and multi-component (bottom insets) best-fit models. See Fig. C.4 for details. 

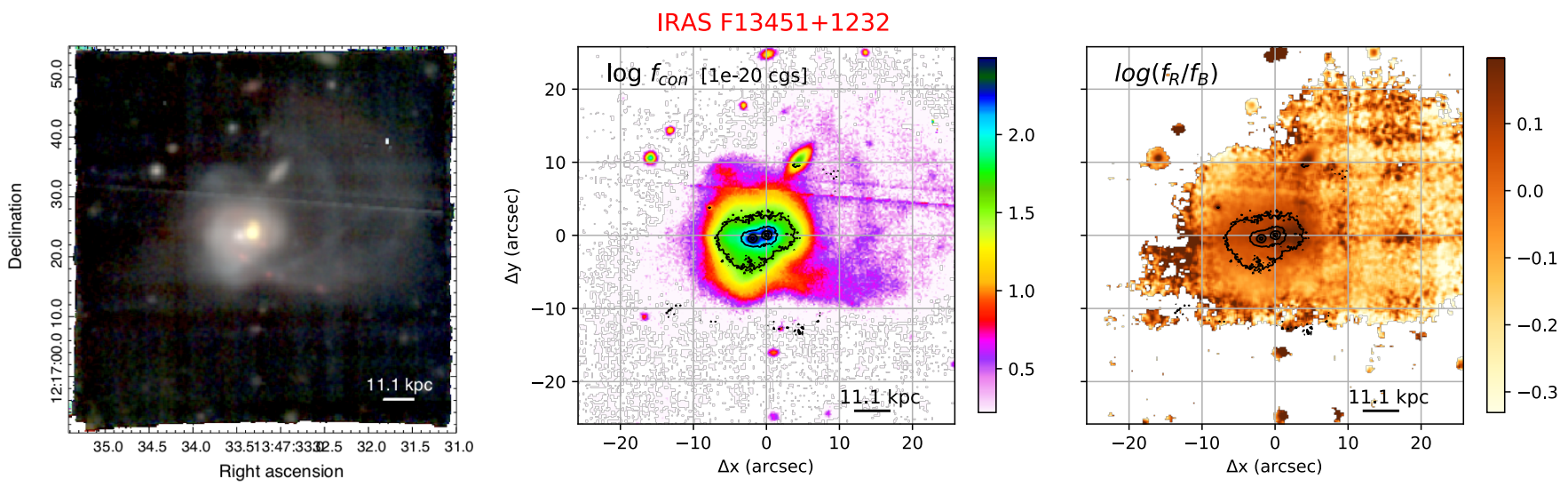

Fig. C.45. IRAS F13451+1232 images from MUSE observations with TOT $=2.04 \mathrm{hr}$. Left: Colour-composite optical image, showing [O III]

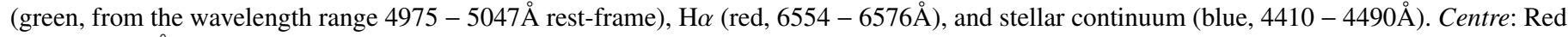

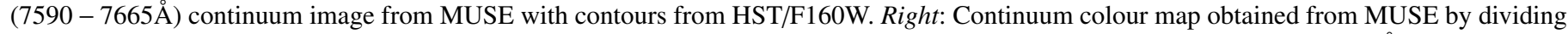
the red continuum image (central panel) by a blue image obtained by collapsing the stellar emission in the range $4410-4490 \AA$; contours from HST/F160W. In all panels, the top part of the ULIRG shows an almost horizontal feature that is due to the removal of a satellite trail.
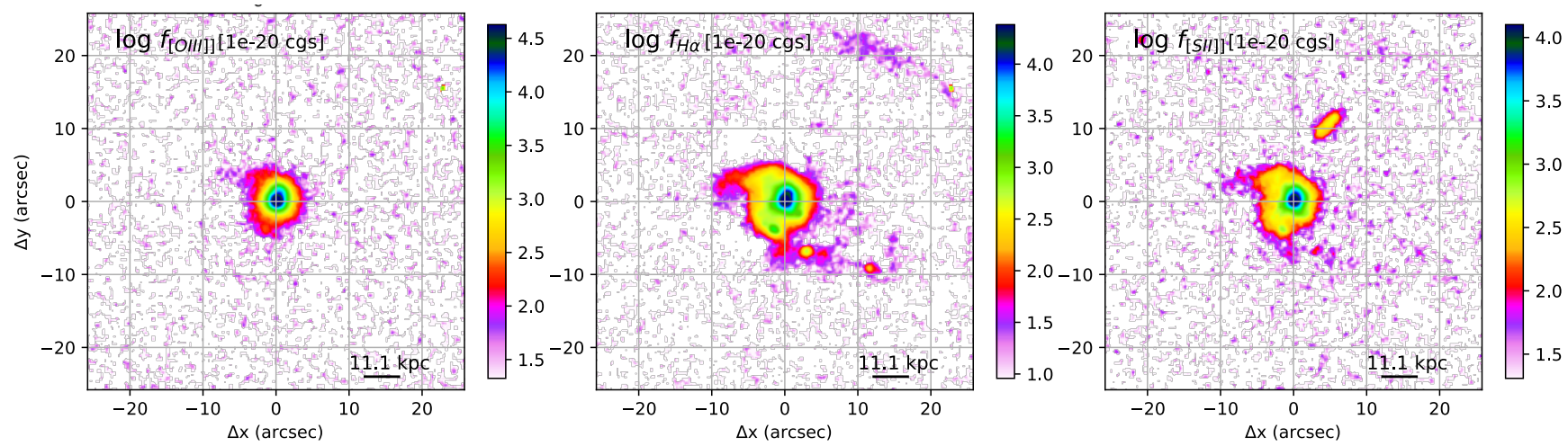

Fig. C.46. IRAS F13451+1232 emission line images from MUSE observations. [O III] (left, from the wavelength range 4975-5047 ̊ rest-frame), $\mathrm{H} \alpha$ (centre, $6554-6576 \AA$ ), and [S II] (right, $6662-6757 \AA$ ) images have been obtained by subtracting continuum emission using the adjacent regions at shorter and longer wavelengths with respect to the emission line systemics.
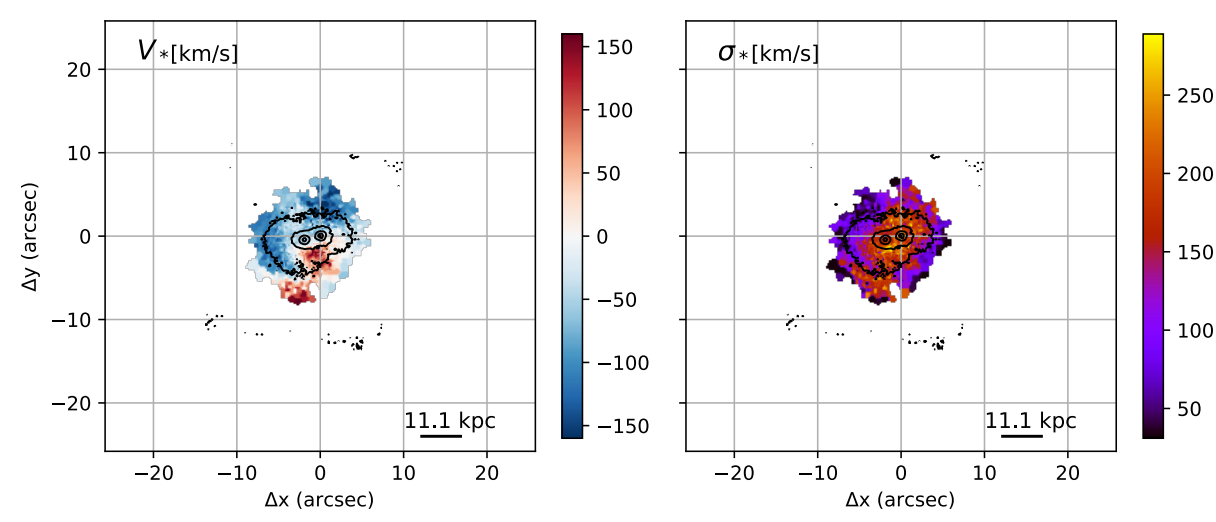

Fig. C.47. IRAS F13451+1232 stellar kinematic maps from the pPXF analysis with contours from HST/F160W. The left panel shows the stellar velocity $V_{*}$, and the right panel represents the velocity dispersion $\sigma_{*}$. 

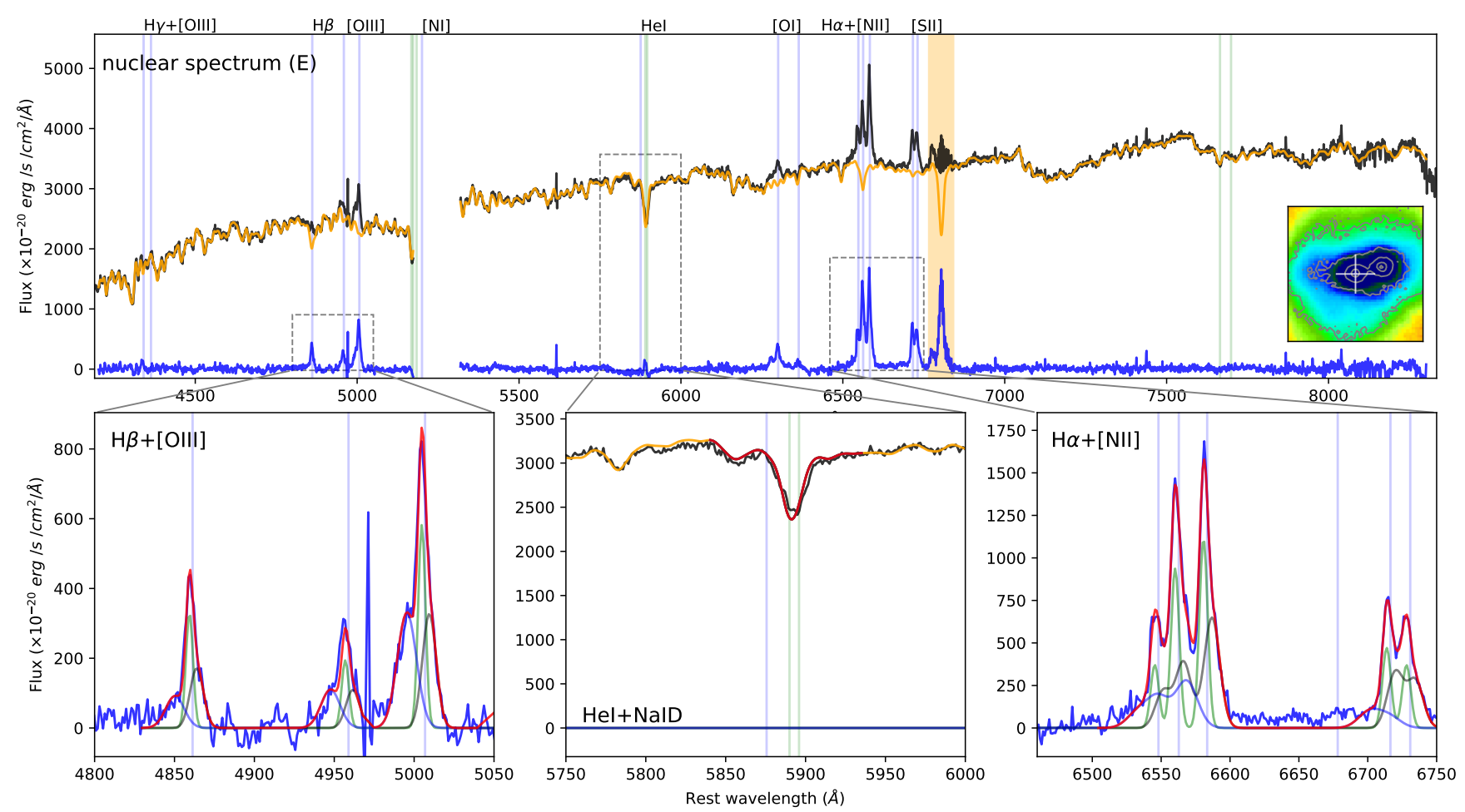

Fig. C.48. IRAS F13451+1232 E nuclear spectrum extracted from a circular aperture with $r<0.4^{\prime \prime}$, with the corresponding pPXF (top panel) and multi-component (bottom insets) best-fit models. See Fig. C.4 for details.

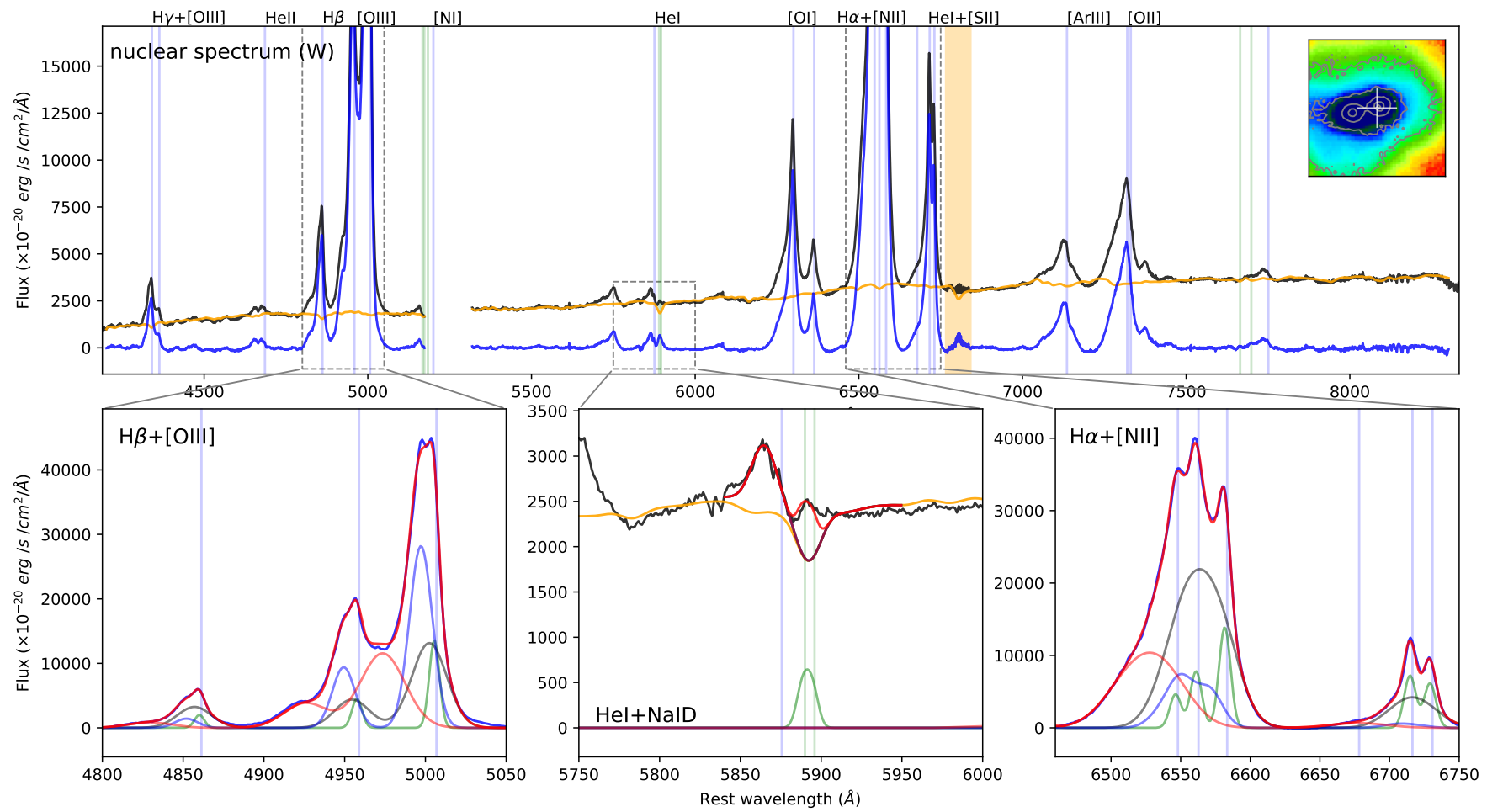

Fig. C.49. IRAS F13451+1232 W nuclear spectrum extracted from a circular aperture with $r<0.4^{\prime \prime}$, with the corresponding pPXF (top panel) and multi-component (bottom insets) best-fit models. See Fig. C.4 for details. 

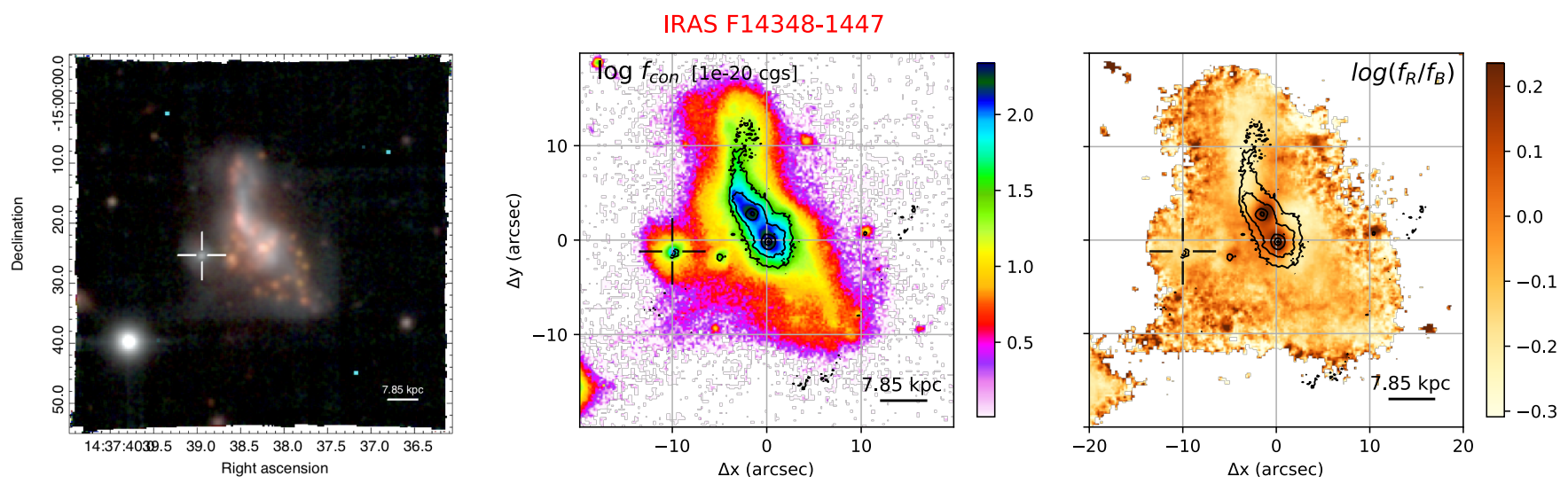

Fig. C.50. IRAS F14348-1447 images from MUSE observations with TOT = $0.68 \mathrm{hr}$. Left: Colour-composite optical image showing [O III] (green,

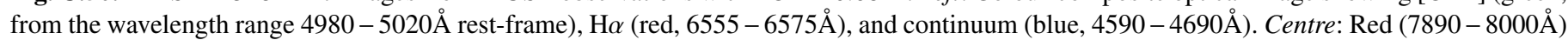
continuum image from MUSE with contours from HST/F160W. Right: Continuum colour map obtained from MUSE by dividing the red continuum image (central panel) by a blue image obtained by collapsing the stellar emission in the range $4590-4690 \AA$; contours from HST/F160W. In all panels, we display the IRAS F14348-1447 companion galaxy with a cross.
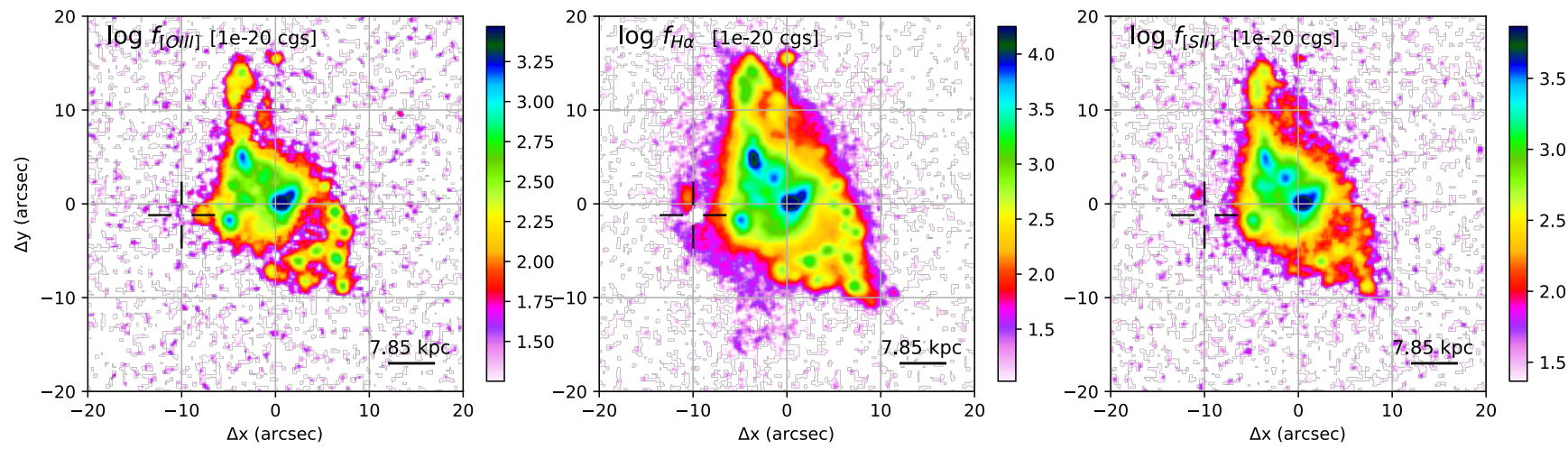

Fig. C.51. IRAS F14348-1447 emission line images from MUSE observations. [O III] (left, from the wavelength range $4980-5020 \AA$ rest-frame), $\mathrm{H} \alpha$ (centre, $6555-6575 \AA$ ), and [S II] (right, $6686-6750 \AA$ ) images have been obtained by subtracting continuum emission using the adjacent regions at shorter and longer wavelengths with respect to the emission line systemics. In all panels, we display the position of the companion galaxy with a cross; the line emission in the three maps is absent because they are faint.
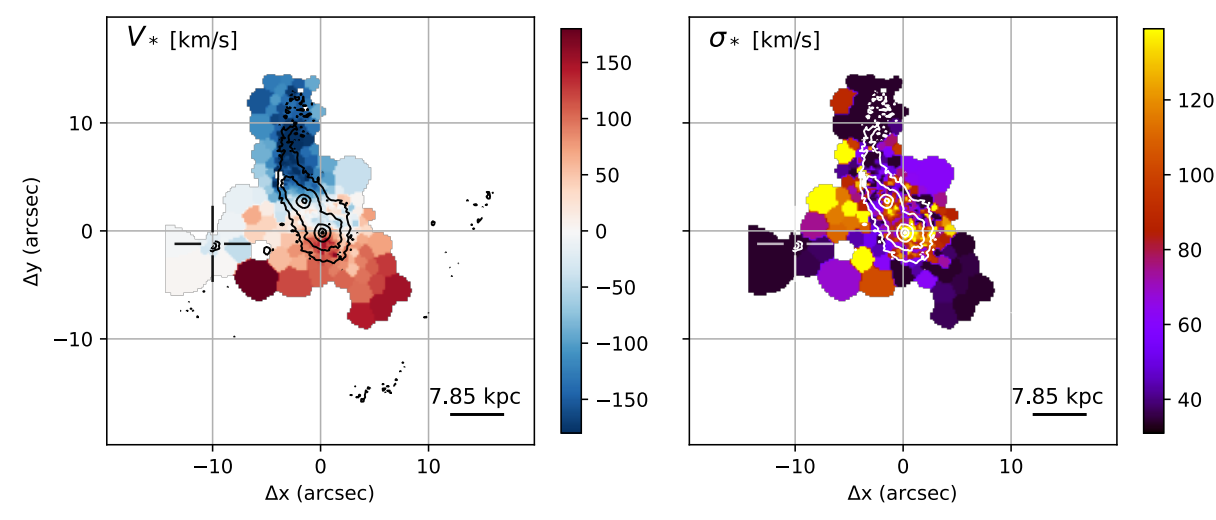

Fig. C.52. IRAS F14348-1447 stellar kinematic maps from the pPXF analysis with contours from HST/F160W. The left panel shows the stellar velocity $V_{*}$, and the right panel represents the velocity dispersion $\sigma_{*}$. 


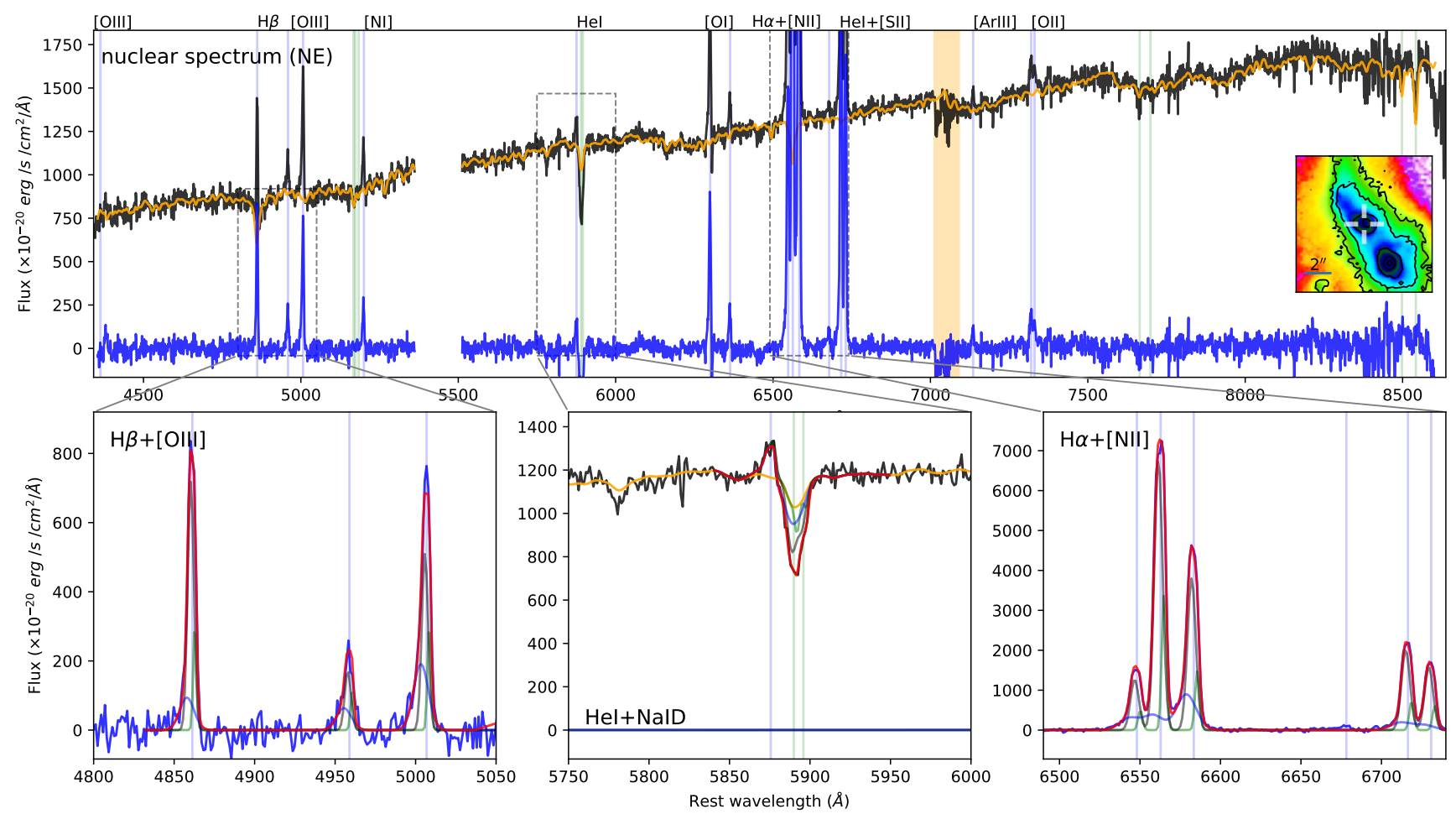

Fig. C.53. IRAS F14348-1447 NE nuclear spectrum extracted from a circular aperture with $r<0.4^{\prime \prime}$, with the corresponding pPXF (top panel) and multi-component (bottom insets) best-fit models. See Fig. C.4 for details.

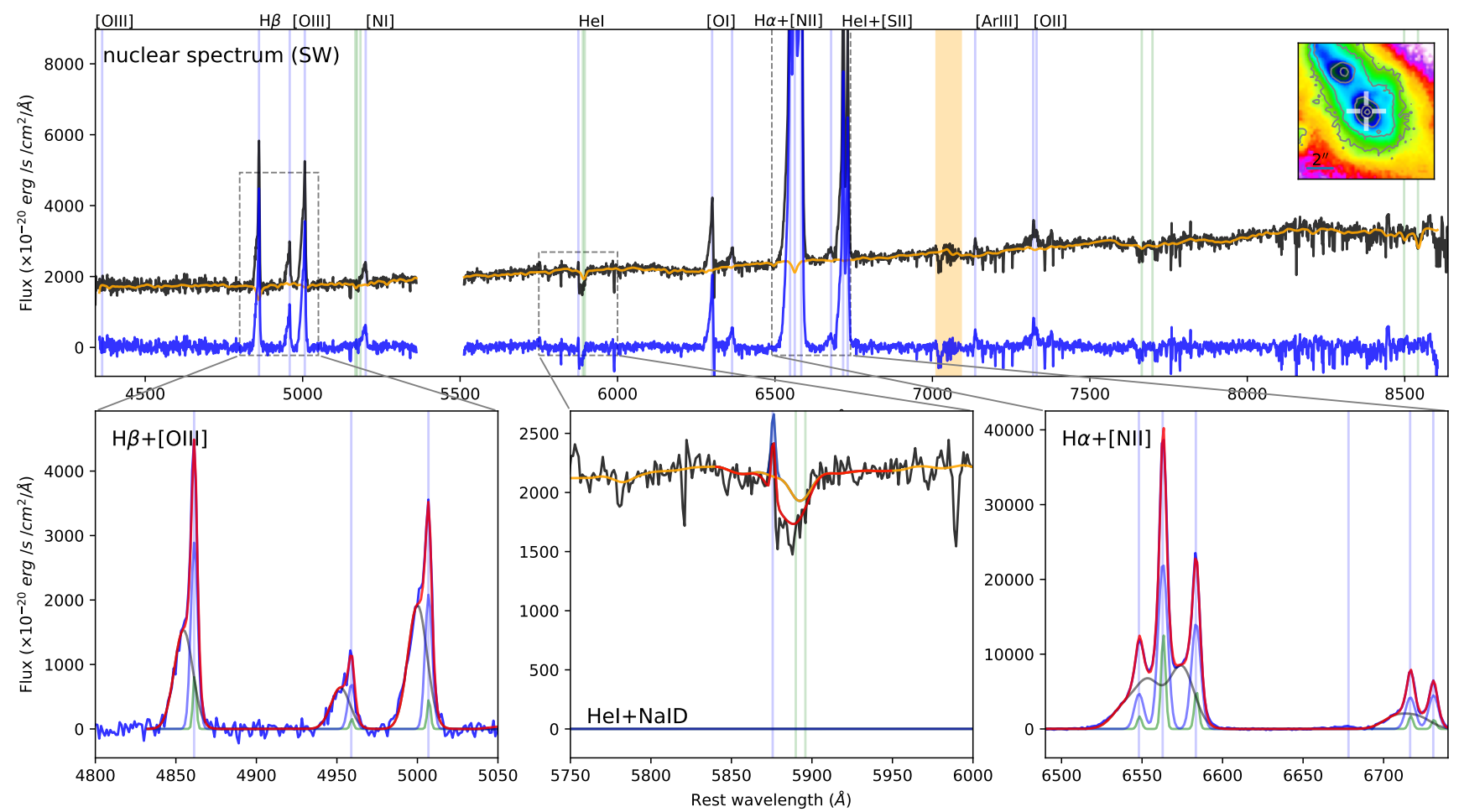

Fig. C.54. IRAS F14348-1447 SW nuclear spectrum extracted from a circular aperture with $r<0.4^{\prime \prime}$, with the corresponding pPXF (top panel) and multi-component (bottom insets) best-fit models. See Fig. C.4 for details. 

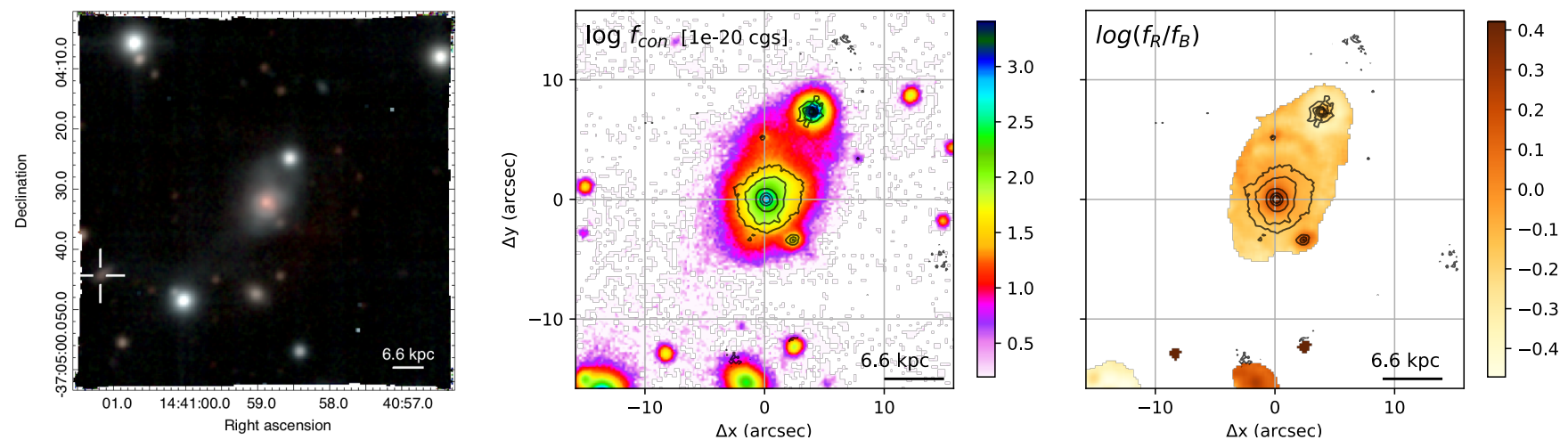

Fig. C.55. IRAS F14378-3651 images from MUSE-noAO observations with TOT = 0.47 hr. Left: Colour-composite optical image showing [O III]

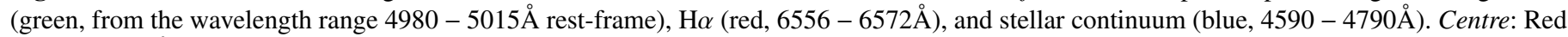
$(7500-7600 \AA)$ continuum image from MUSE with contours from HST/F160W. Right: Continuum colour map obtained from MUSE by dividing the red continuum image (central panel) by a blue image obtained by collapsing the stellar emission in the range $4590-4790 \AA$; contours from HST/F160W. In the left panel, we display the IRAS F14378-3651 companion galaxy with a cross.
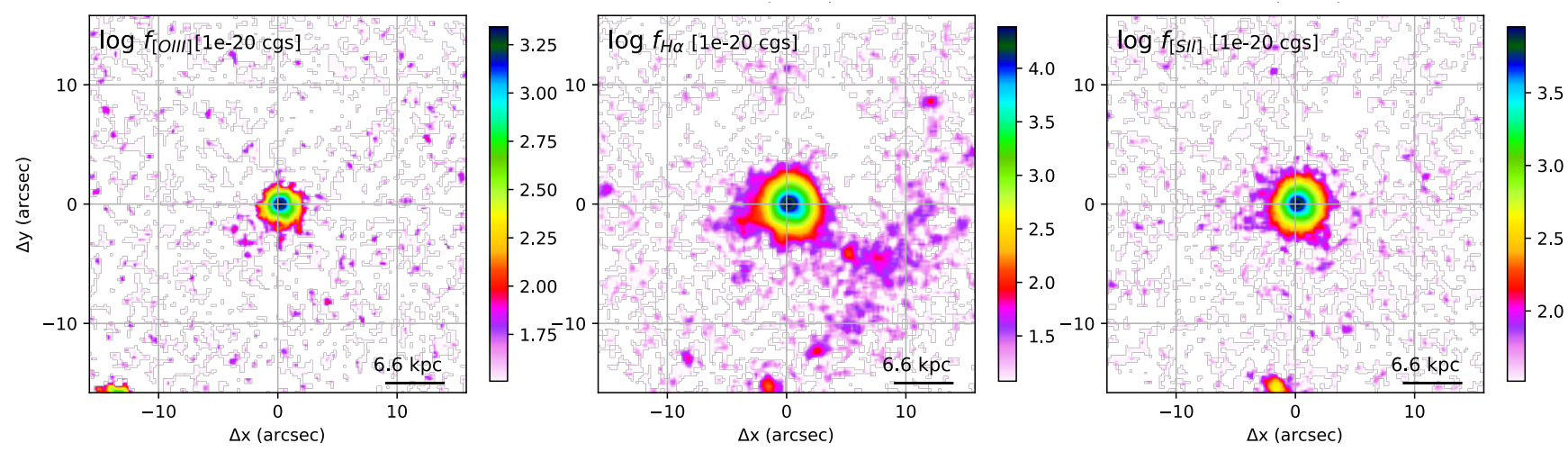

Fig. C.56. IRAS F14378-3651 emission line images from MUSE observations. [O III] (left, from the wavelength range 4980 - 5015Å rest-frame), $\mathrm{H} \alpha$ (centre, $6556-6572 \AA$ ), and [S II] (right, $6695-6747 \AA$ ) images have been obtained by subtracting continuum emission using the adjacent regions at shorter and longer wavelengths with respect to the emission line systemics.
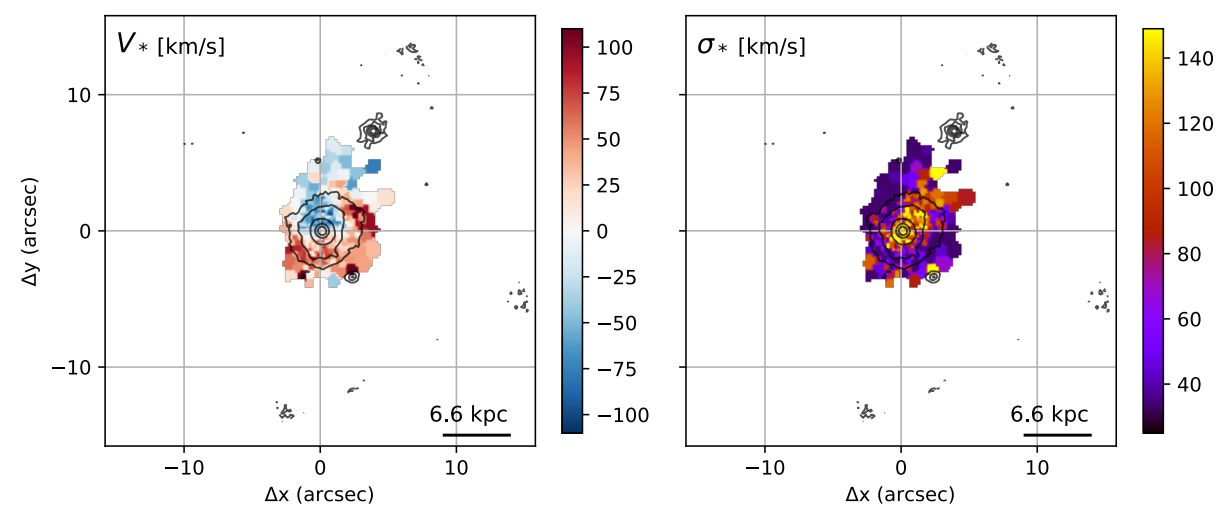

Fig. C.57. IRAS F14378-3651 stellar kinematic maps from the pPXF analysis with contours from HST/F160W. The left panel shows the stellar velocity $V_{*}$, and the right panel represents the velocity dispersion $\sigma_{*}$. 

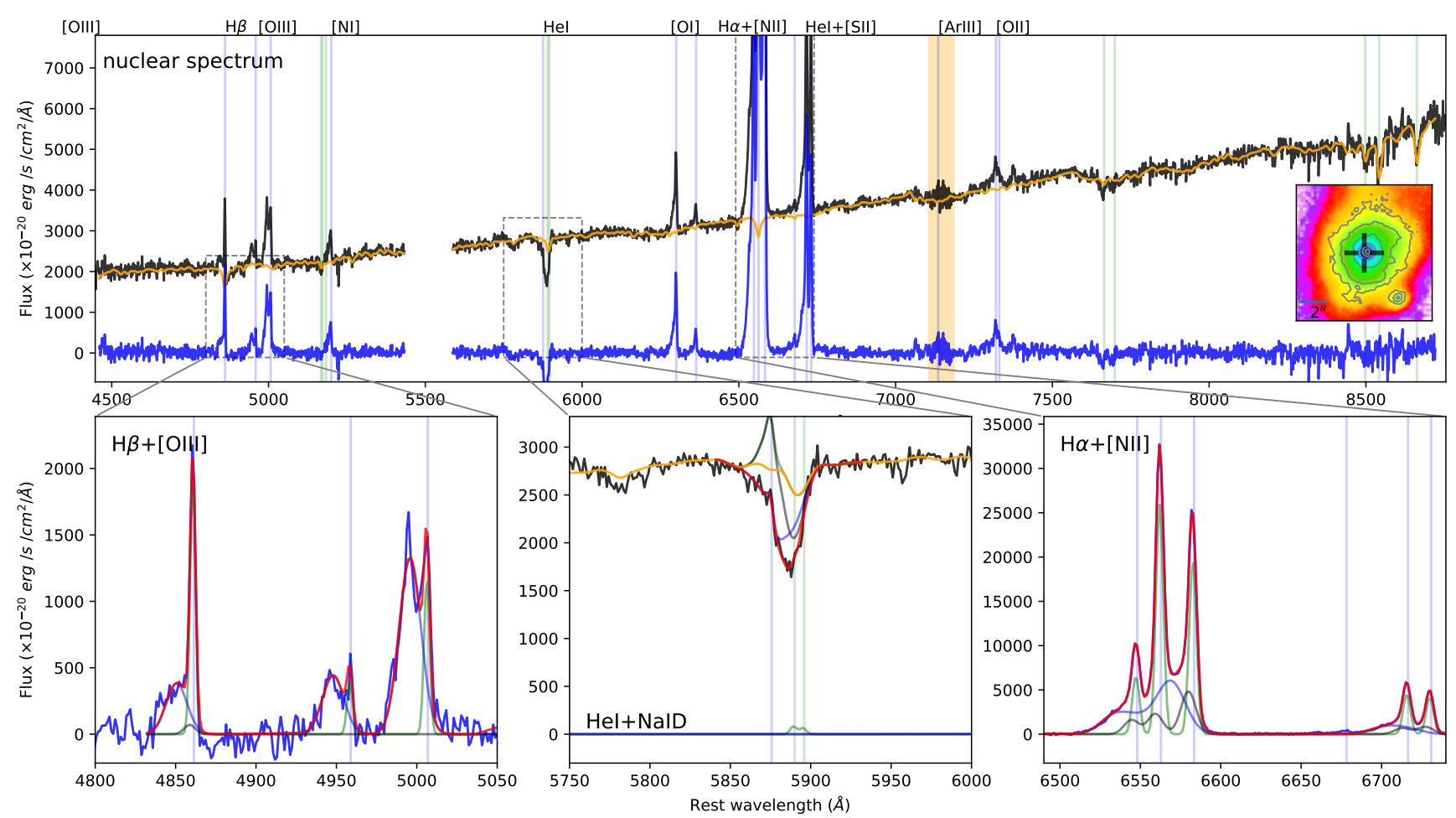

Fig. C.58. IRAS F14378-3651 nuclear spectrum extracted from a circular aperture with $r<0.4^{\prime \prime}$, with the corresponding pPXF (top panel) and multi-component (bottom insets) best-fit models. See Fig. C.4 for details. 

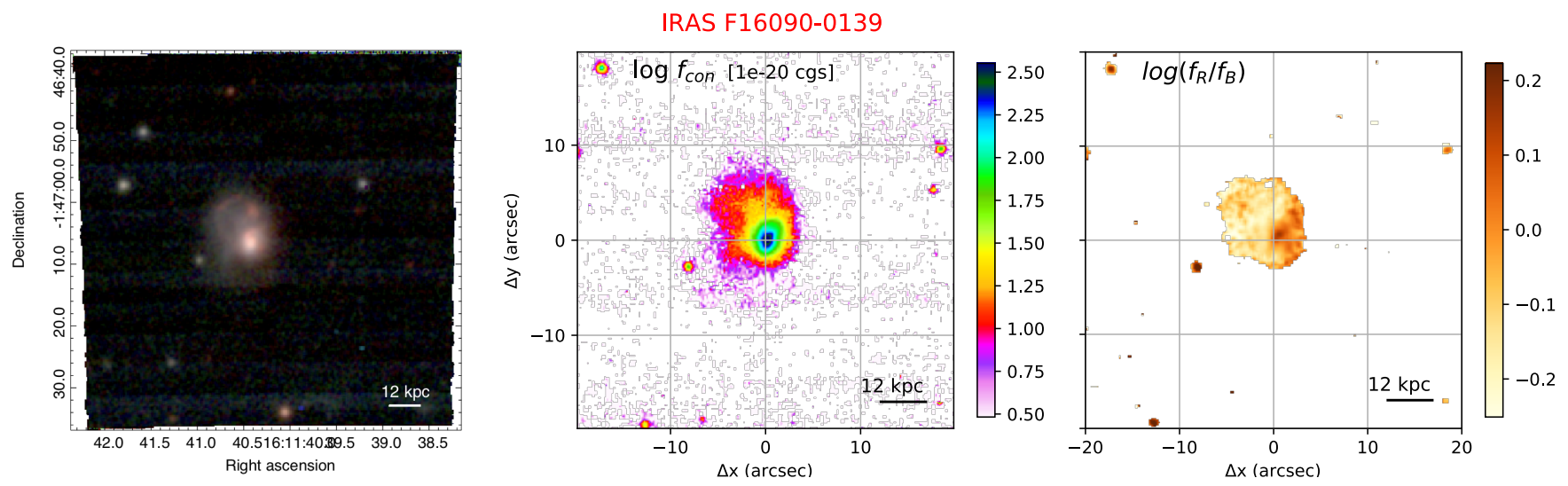

Fig. C.59. IRAS F16090-0139 images from MUSE observations with TOT $=0.17 \mathrm{hr}$. Left: Colour-composite optical image showing [O III] (green, from the wavelength range $4983-5016 \AA$ rest-frame), $\mathrm{H} \alpha$ (red, $6556-6573 \AA$ ), and stellar continuum (blue, $4420-4480 \AA$ ). Centre: Red $(7670-6730 \AA)$ continuum image from MUSE with contours from HST/F160W. Right: Stellar continuum colour map obtained from MUSE by dividing the red continuum image (central panel) by a blue image obtained by collapsing the stellar emission in the range $4420-4480 \AA$.
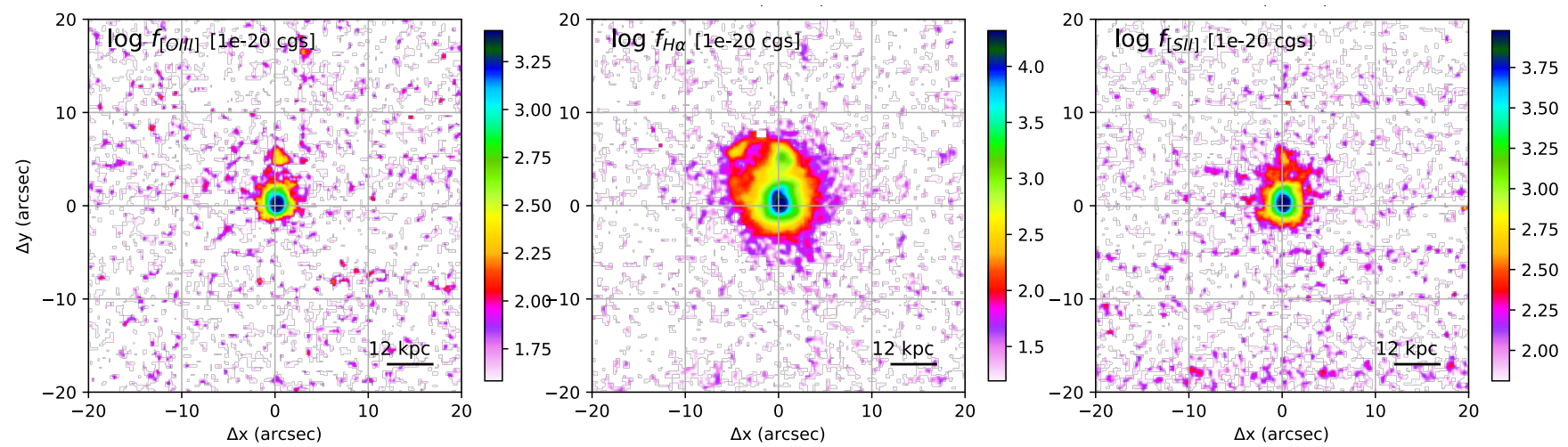

Fig. C.60. IRAS F16090-0139 emission line images from MUSE observations. [O III] (left, from the wavelength range 4983 - 5016 $\mathrm{A}$ rest-frame), $\mathrm{H} \alpha$ (centre, $6556-6573 \AA$ ), and [S II] (right, $6690-6747 \AA$ ) images have been obtained by subtracting continuum emission using the adjacent regions at shorter and longer wavelengths with respect to the emission line systemics.
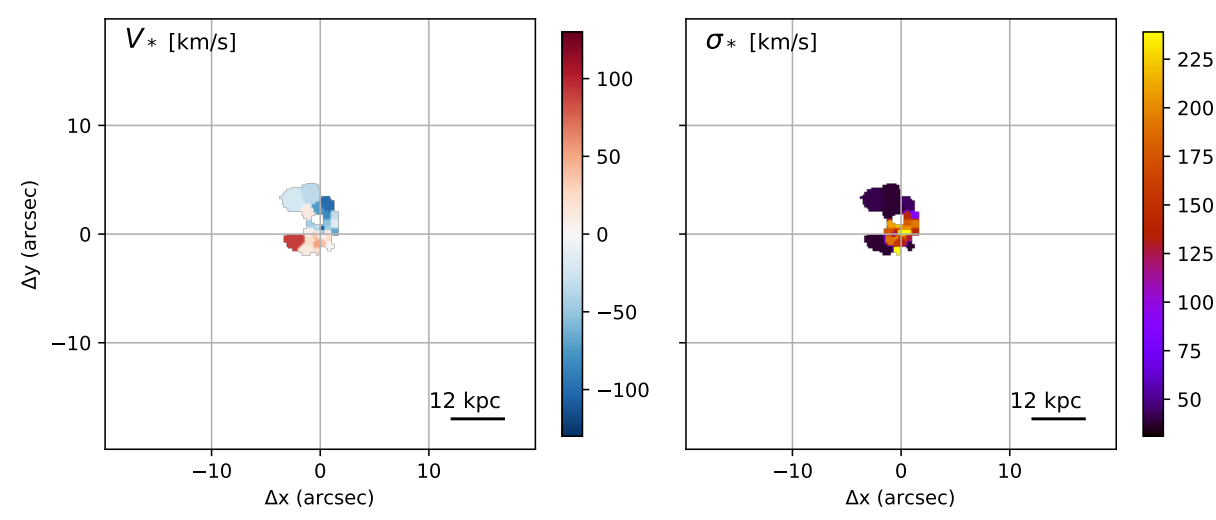

Fig. C.61. IRAS F16090-0139 stellar kinematic maps from the pPXF analysis with contours from HST/F160W. The left panel shows the stellar velocity $V_{*}$, and the right panel represents the velocity dispersion $\sigma_{*}$. 


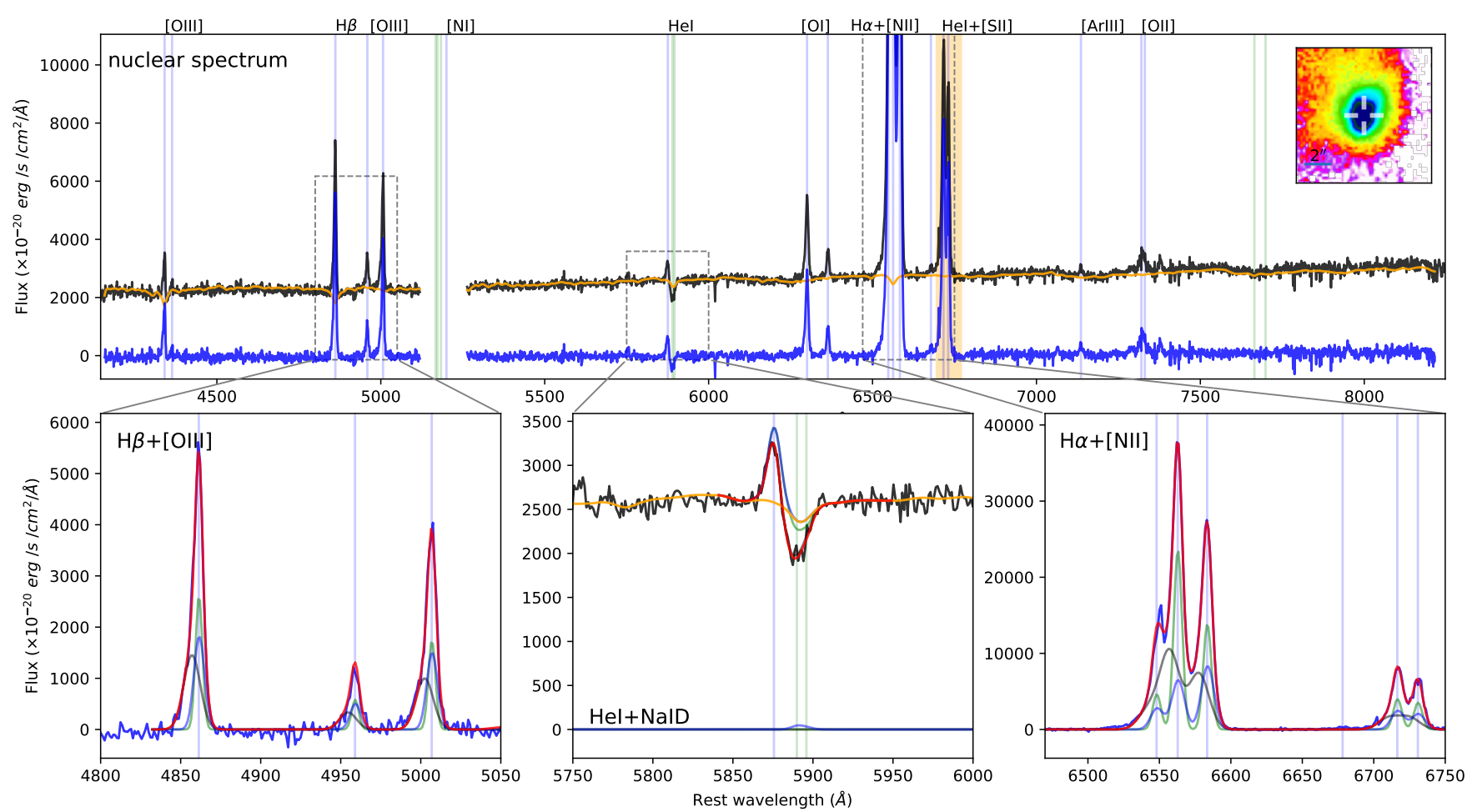

Fig. C.62. IRAS F16090-0139 nuclear spectrum extracted from a circular aperture with $r<0.4^{\prime \prime}$, with the corresponding pPXF (top panel) and multi-component (bottom insets) best-fit models. See Fig. C.4 for details. 

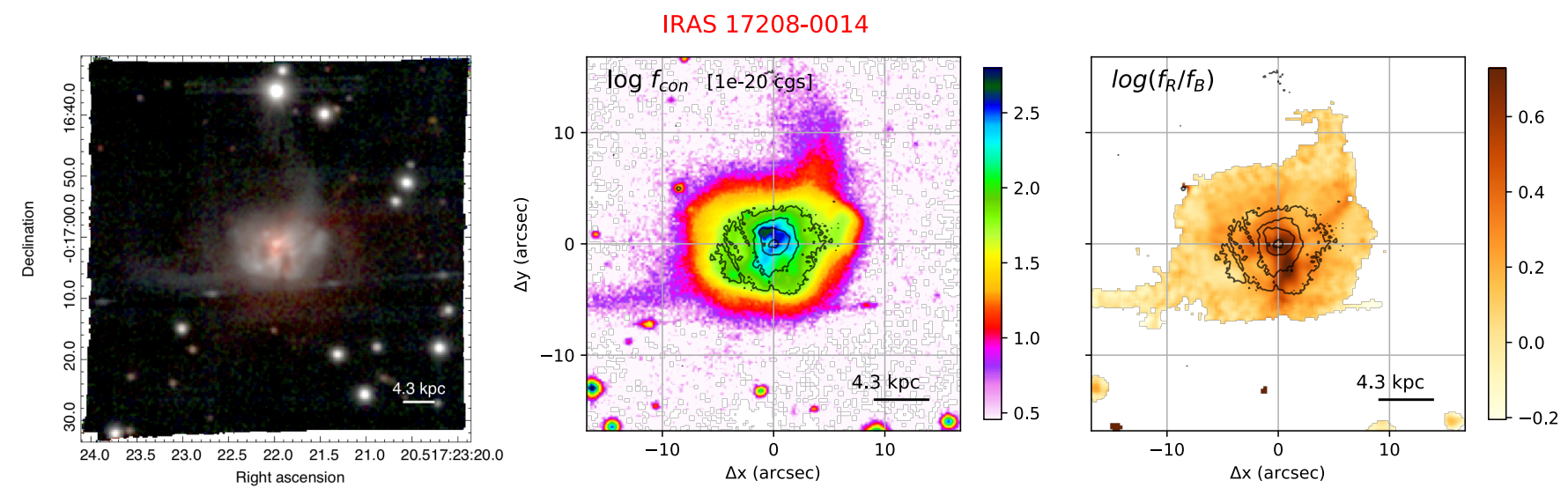

Fig. C.63. IRAS 17208-0014 images from MUSE observations with TOT $=0.39$ hr. Left: Colour-composite optical image showing [O III] (green, from the wavelength range $4975-5032 \AA$ rest-frame), $\mathrm{H} \alpha$ (red, $6549-6576 \AA$ ), and stellar continuum (blue, $4520-4490 \AA$ ). Centre: Red $(7690-7760 \AA)$ continuum image from MUSE with contours from HST/F160W. Right: Stellar continuum colour map obtained from MUSE by dividing the red continuum image (central panel) by a blue image obtained by collapsing the stellar emission in the range $4520-4490 \AA$. In all panels, the bottom part of the ULIRG shows a satellite trail resulting in three artificial blobs in the continuum emission.
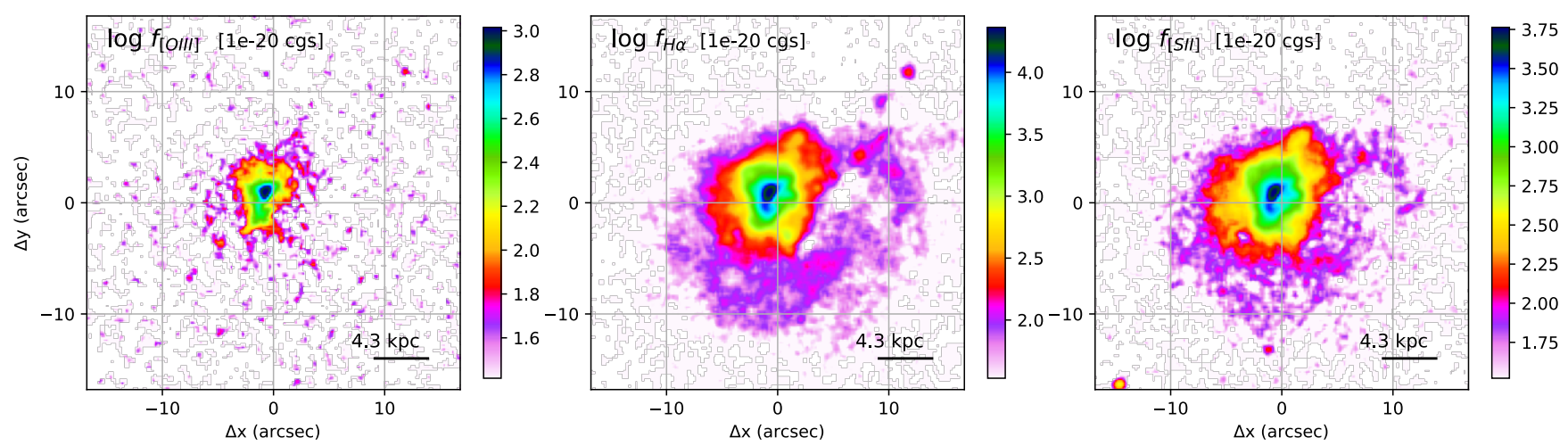

Fig. C.64. IRAS 17208-0014 emission line images from MUSE observations. [O III] (left, from the wavelength range 4975 - 5032Å rest-frame), $\mathrm{H} \alpha$ (centre, $6549-6576 \AA$ ), and [S II] (right, $6695-6749 \AA$ ) images have been obtained by subtracting continuum emission using the adjacent regions at shorter and longer wavelengths with respect to the emission line systemics.
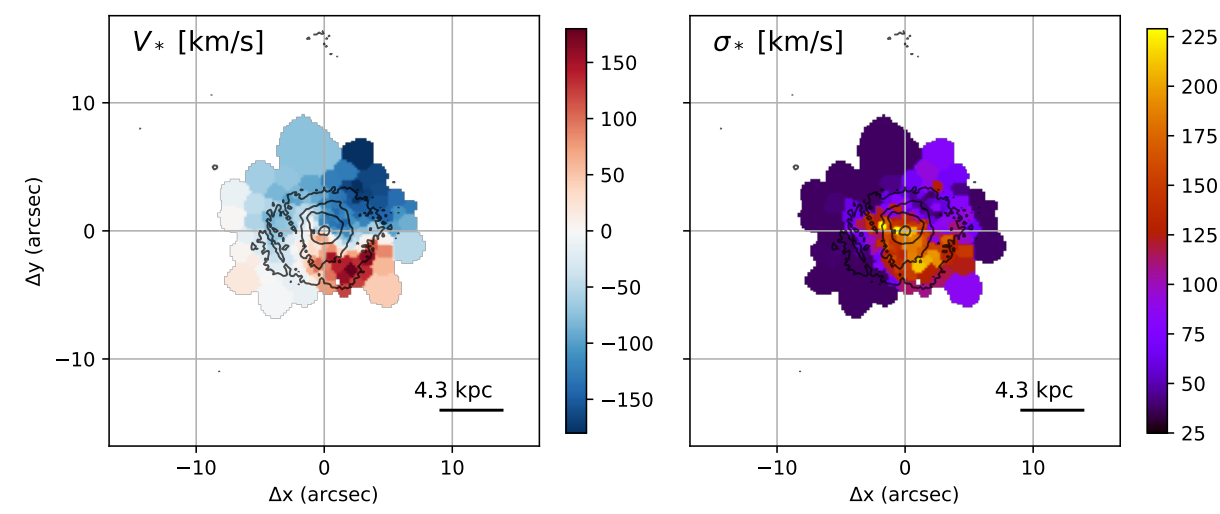

Fig. C.65. IRAS 17208-0014 stellar kinematic maps from the pPXF analysis with contours from HST/F160W. The left panel shows the stellar velocity $V_{*}$, and the right panel represents the velocity dispersion $\sigma_{*}$. 


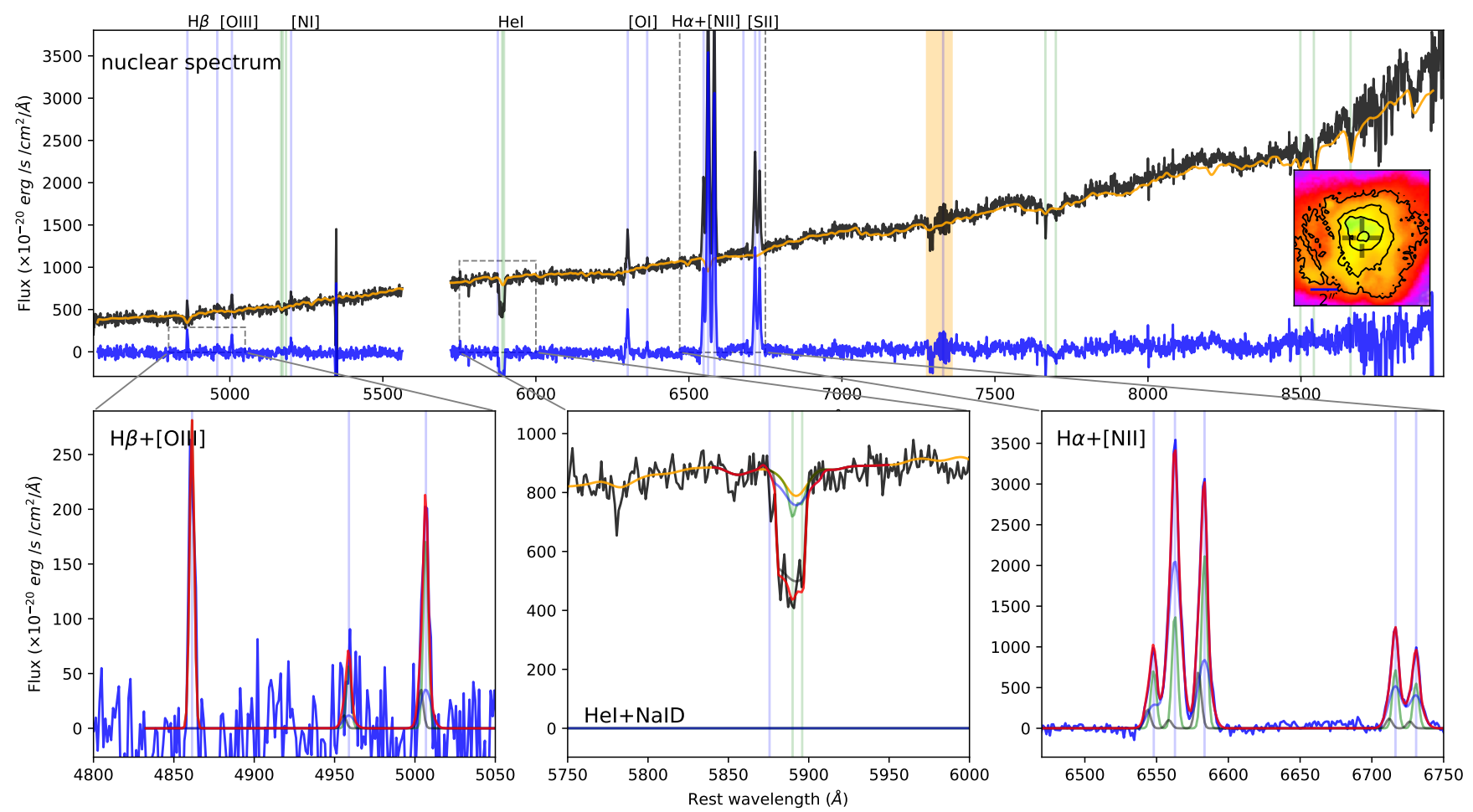

Fig. C.66. IRAS 17208-0014 nuclear spectrum extracted from a circular aperture with $r<0.4^{\prime \prime}$, with the corresponding pPXF (top panel) and multi-component (bottom insets) best-fit models. See Fig. C.4 for details. 

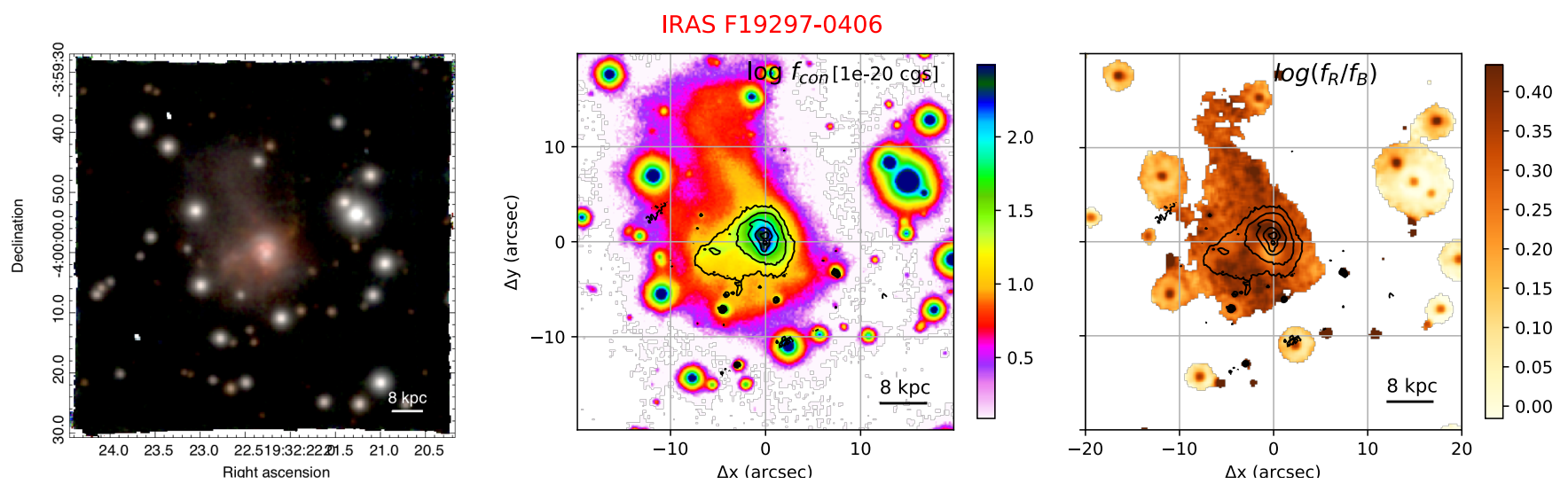

Fig. C.67. IRAS F19297-0406 images from MUSE observations with TOT $=2.04$ hr. Left: Colour-composite optical image showing [O III] (green, from the wavelength range $4991-5021 \AA$ rest-frame), H $\alpha$ (red, $6557-6579 \AA$ ), and stellar continuum (blue, $4550-4620 \AA ̊)$. Centre: Red $(7440-7590 \AA)$ continuum image from MUSE with contours from HST/F160W. Right: Stellar continuum colour map obtained from MUSE by dividing the red continuum image (central panel) by a blue image obtained by collapsing the stellar emission in the range $4550-4620 \AA$.
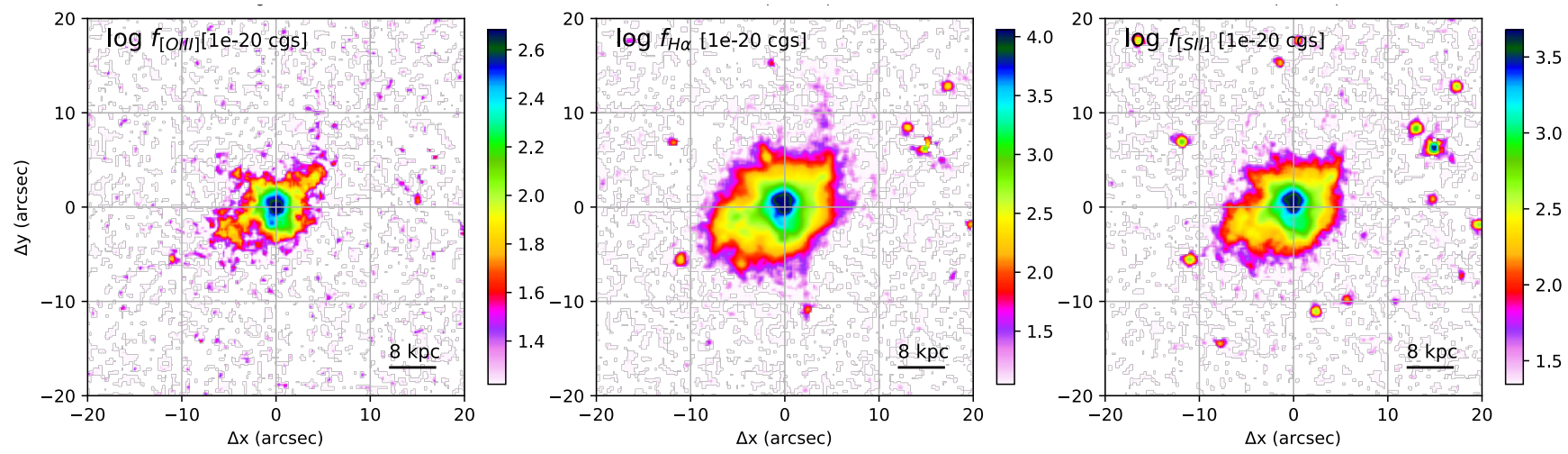

Fig. C.68. IRAS F19297-0406 emission line images from MUSE observations. [O III] (left, from the wavelength range $4991-5021 \AA ̊$ rest-frame), $\mathrm{H} \alpha$ (centre, $6557-6579 \AA$ ), and [S II] (right, $6700-6752 \AA$ ) images have been obtained by subtracting continuum emission using the adjacent regions at shorter and longer wavelengths with respect to the emission line systemics.
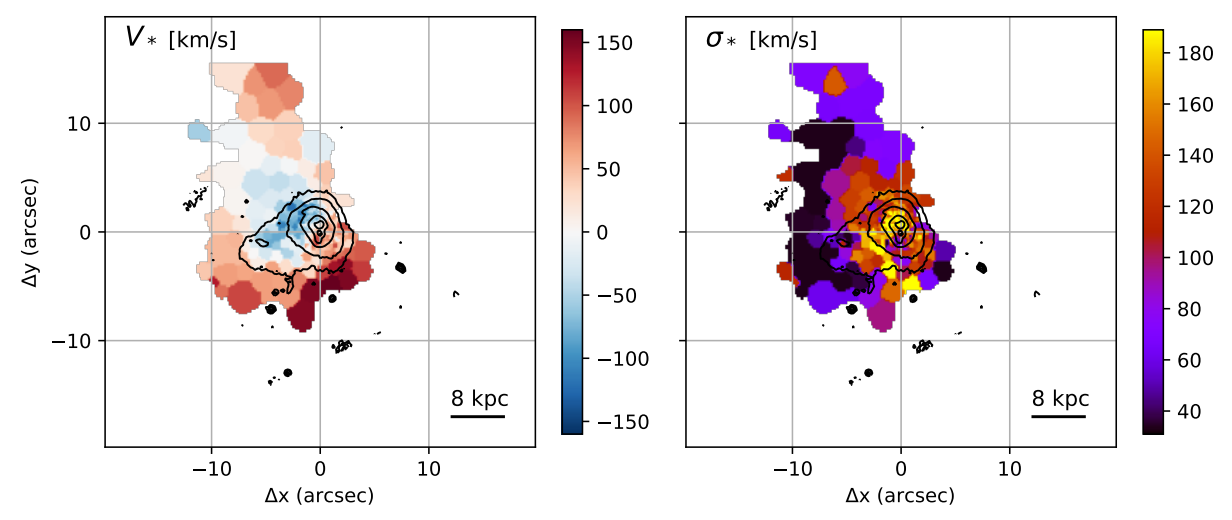

Fig. C.69. IRAS F19297-0406 stellar kinematic maps from the pPXF analysis with contours from HST/F160W. The left panel shows the stellar velocity $V_{*}$, and the right panel represents the velocity dispersion $\sigma_{*}$. 


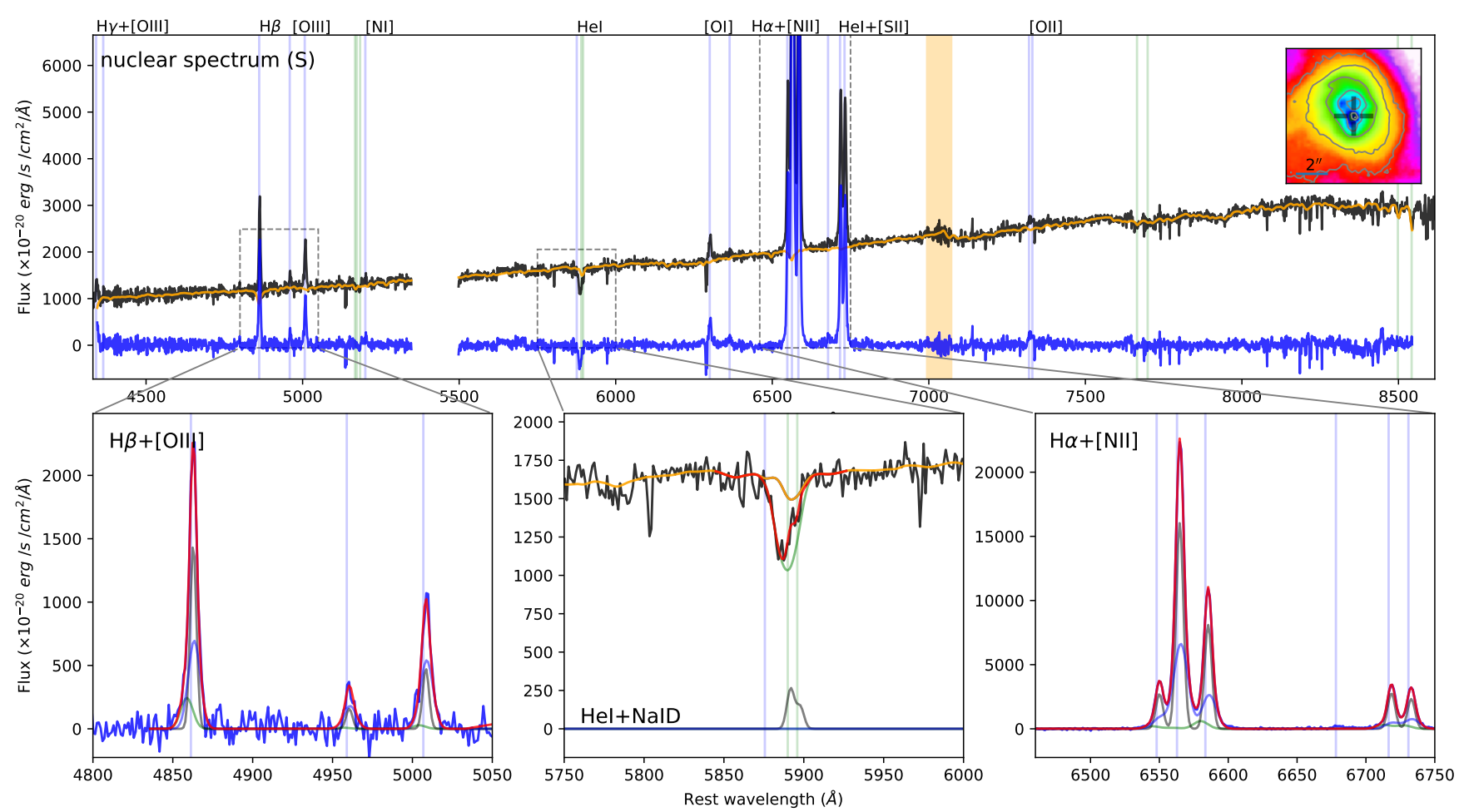

Fig. C.70. IRAS F19297-0406 (S) nuclear spectrum extracted from a circular aperture with $r<0.4^{\prime \prime}$, with the corresponding pPXF (top panel) and multi-component (bottom insets) best-fit models. See Fig. C.4 for details.

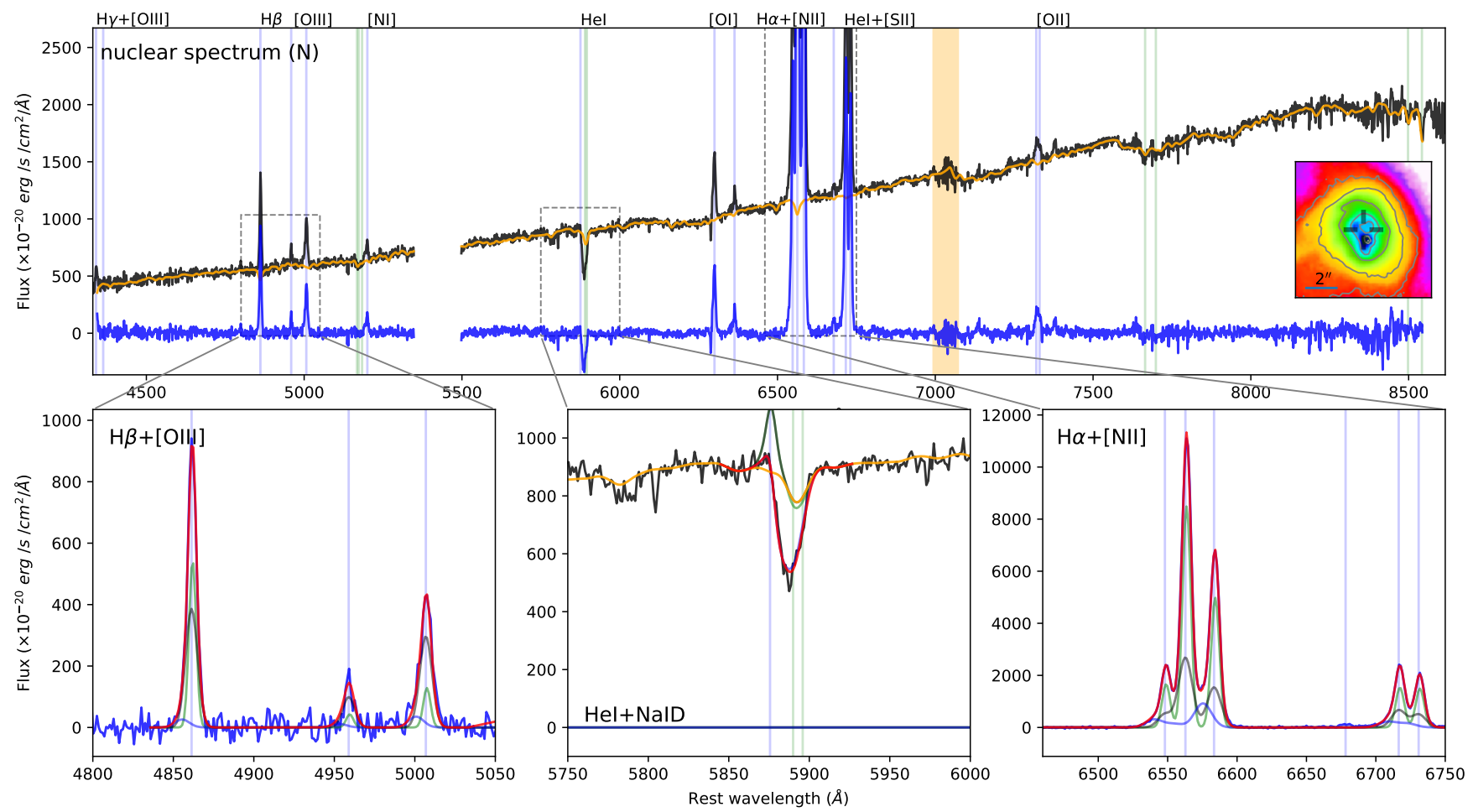

Fig. C.71. IRAS F19297-0406 (N) nuclear spectrum extracted from a circular aperture with $r<0.4^{\prime \prime}$, with the corresponding pPXF (top panel) and multi-component (bottom insets) best-fit models. See Fig. C.4 for details. 

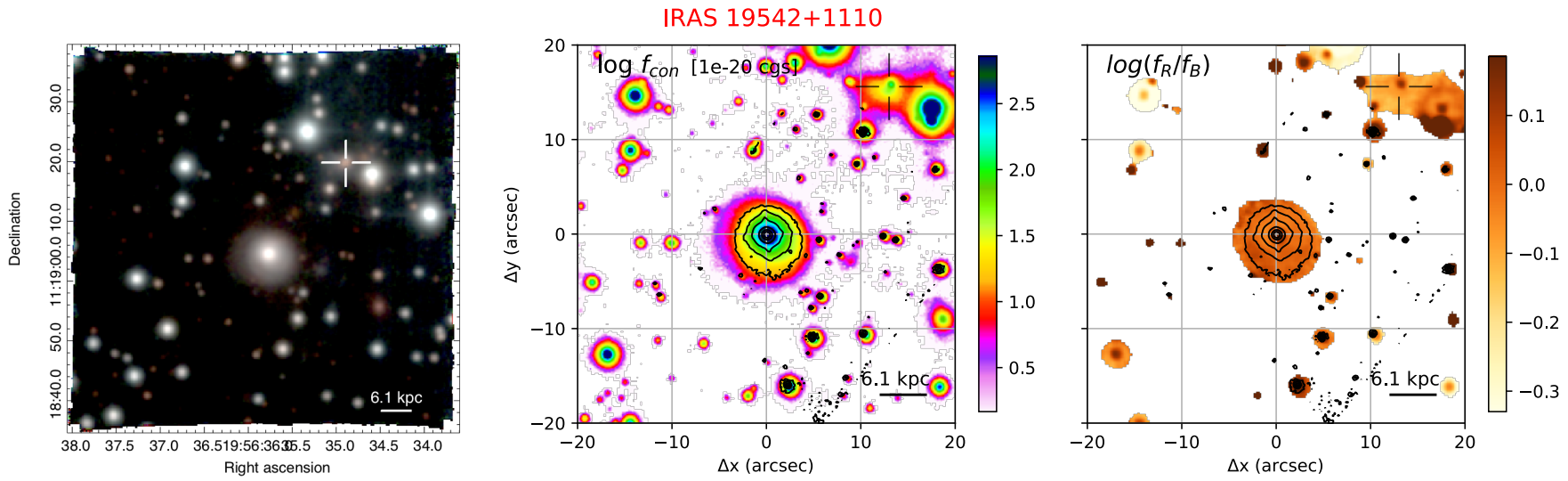

Fig. C.72. IRAS $19542+1110$ images from MUSE observations with TOT $=2.04 \mathrm{hr}$. Left: Colour-composite optical image showing [O III] (green, from the wavelength range $4985-5016 \AA$ rest-frame), $\mathrm{H} \alpha$ (red, $6552-6573 \AA$ ), and stellar continuum (blue, $4630-4740 \AA$ ). Centre: Red (7650 - 7750Å) continuum image from MUSE with contours from HST/F160W. Right: Stellar continuum colour map obtained from MUSE by dividing the red continuum image (central panel) by a blue image obtained by collapsing the continuum emission in the range $4630-4740 \AA$; contours from HST/F160W. In all panels, we display the companion galaxy of IRAS 19542+1110 with a cross.
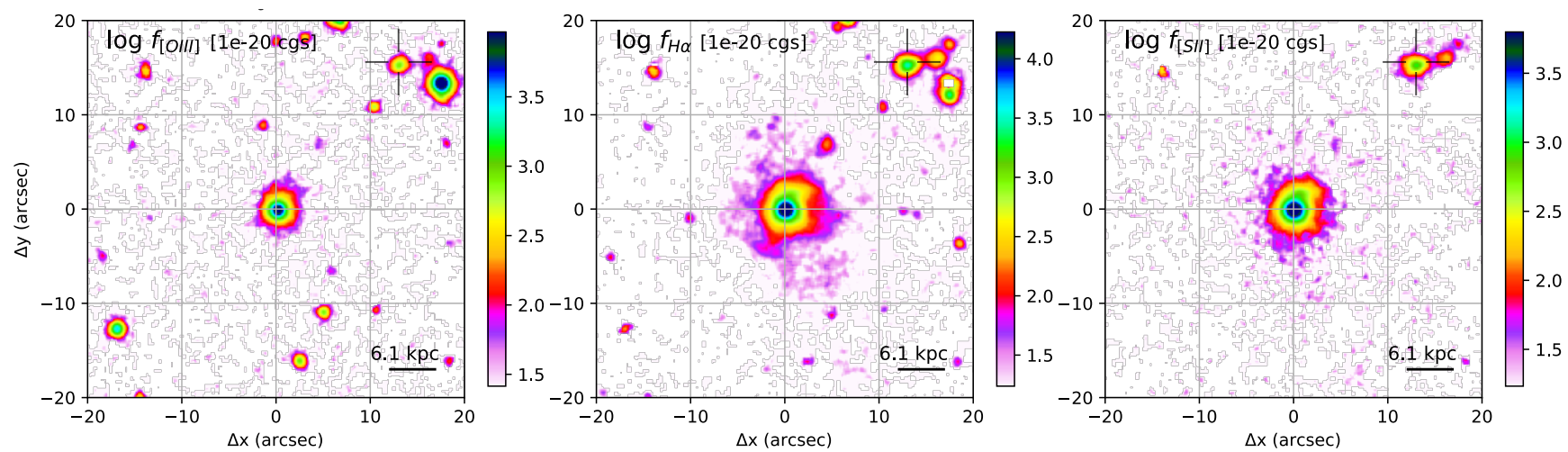

Fig. C.73. IRAS 19542+1110 emission line images from MUSE observations. [O III] (left, from the wavelength range 4985 - 5016 ̊ rest-frame), $\mathrm{H} \alpha$ (centre, $6552-6573 \AA$ ), and [S II] (right, $6700-6744 \AA$ ) images have been obtained by subtracting continuum emission using the adjacent regions at shorter and longer wavelengths with respect to the emission line systemics.
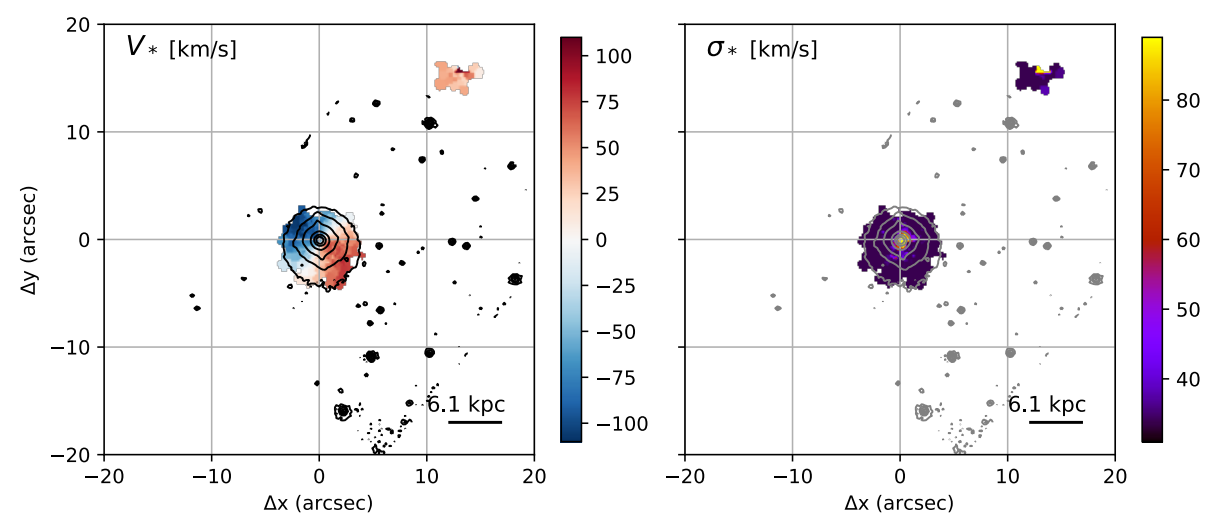

Fig. C.74. IRAS 19542+1110 stellar kinematic maps from the pPXF analysis with contours from HST/F160W. The left panel shows the stellar velocity $V_{*}$, and the right panel represents the velocity dispersion $\sigma_{*}$. 


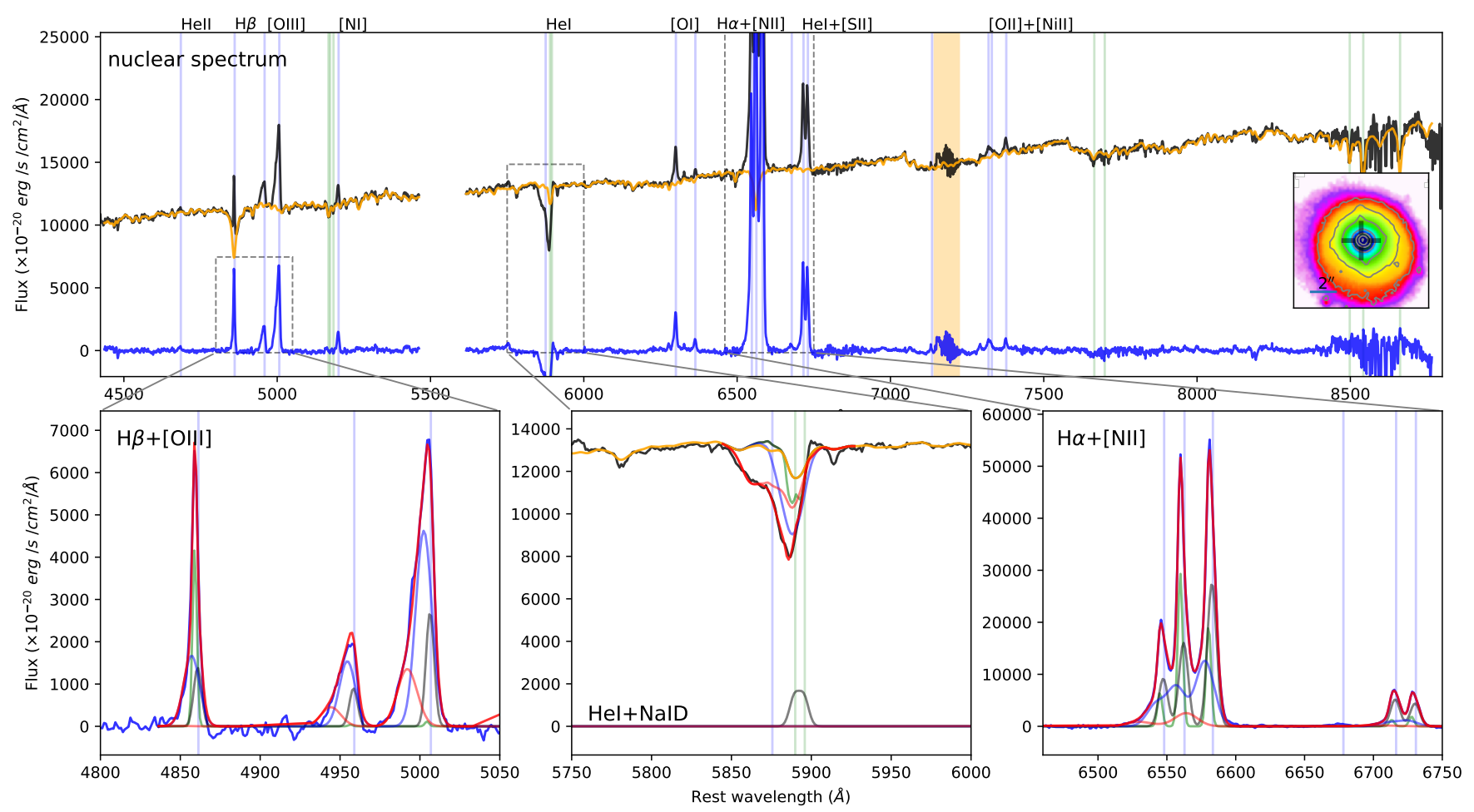

Fig. C.75. IRAS 19542+1110 nuclear spectrum extracted from a circular aperture with $r<0.4^{\prime \prime}$, with the corresponding pPXF (top panel) and multi-component (bottom insets) best-fit models. See Fig. C.4 for details. 

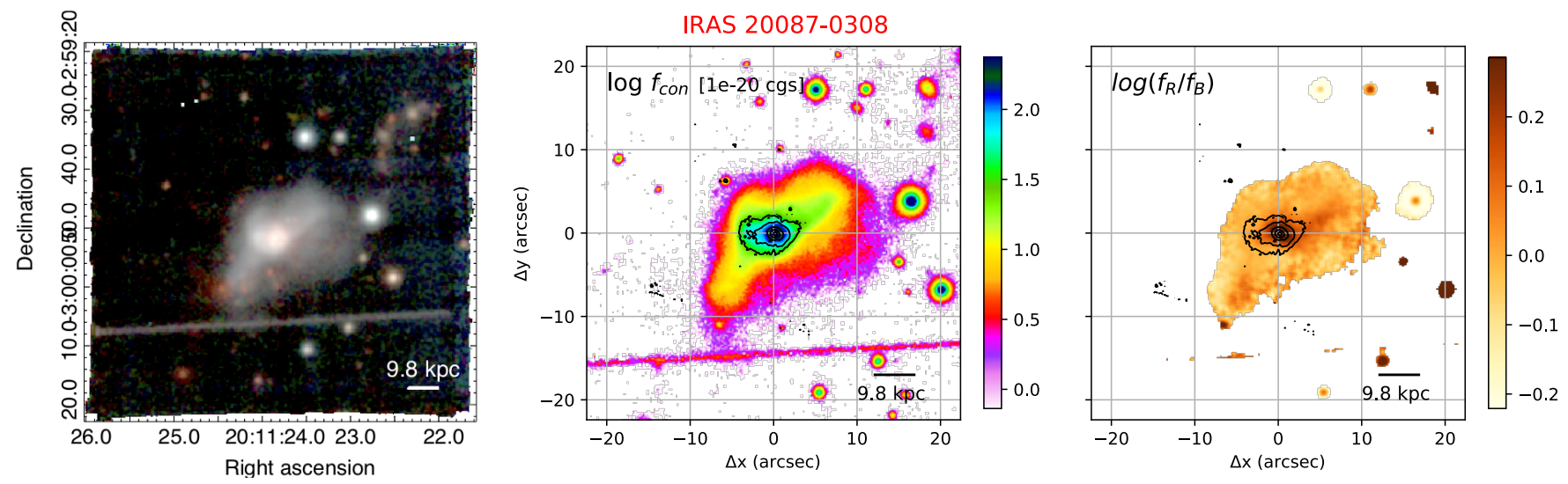

Fig. C.76. IRAS 20087-0308 images from MUSE observations with TOT = $2.04 \mathrm{hr}$. Left: Colour-composite optical image showing [O III] (green, from the wavelength range $4986-5020 \AA$ rest-frame), $\mathrm{H} \alpha$ (red, $6555-6574 \AA$ ), and continuum (blue, $4600-4700 \AA$ ). Centre: Red (7780 - 7880 $)$ continuum image from MUSE with contours from HST/F160W. Right: Stellar continuum colour map obtained from MUSE by dividing the red continuum image (central panel) by a blue image obtained by collapsing the stellar emission in the range 4600-4700Å; contours from HST/F160W. In all panels, the bottom part of the FOV shows a satellite trail.
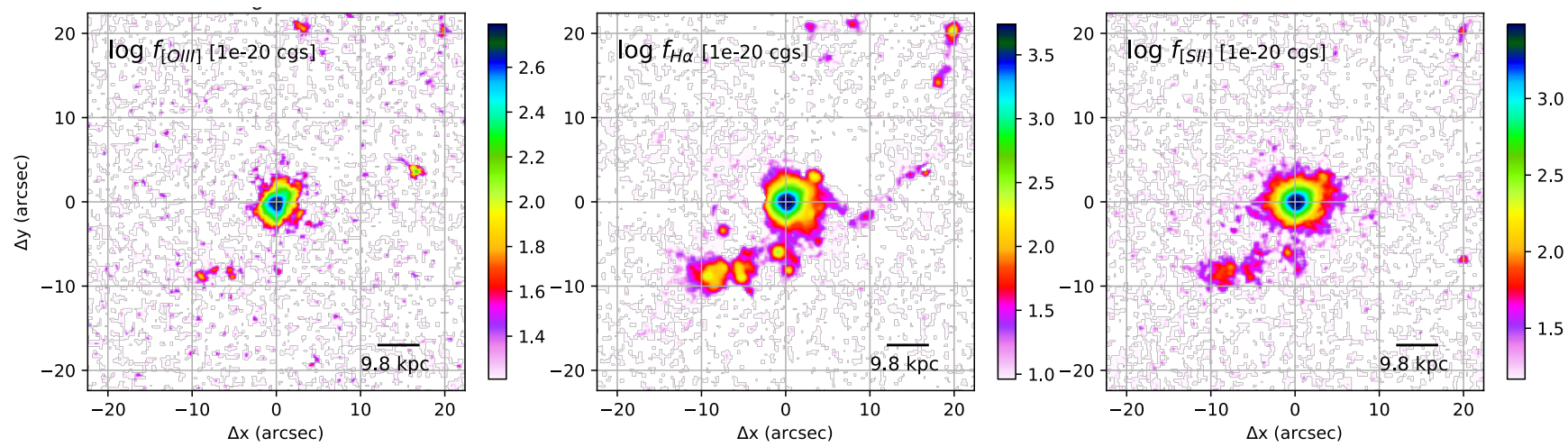

Fig. C.77. IRAS 20087-0308 emission line images from MUSE observations. [O III] (left, from the wavelength range 4986 - 5020 $\mathrm{A}$ rest-frame), $\mathrm{H} \alpha$ (centre, $6555-6574 \AA$ ), and [S II] (right, $6700-6747 \AA$ ) images have been obtained by subtracting continuum emission using the adjacent regions at shorter and longer wavelengths with respect to the emission line systemics.
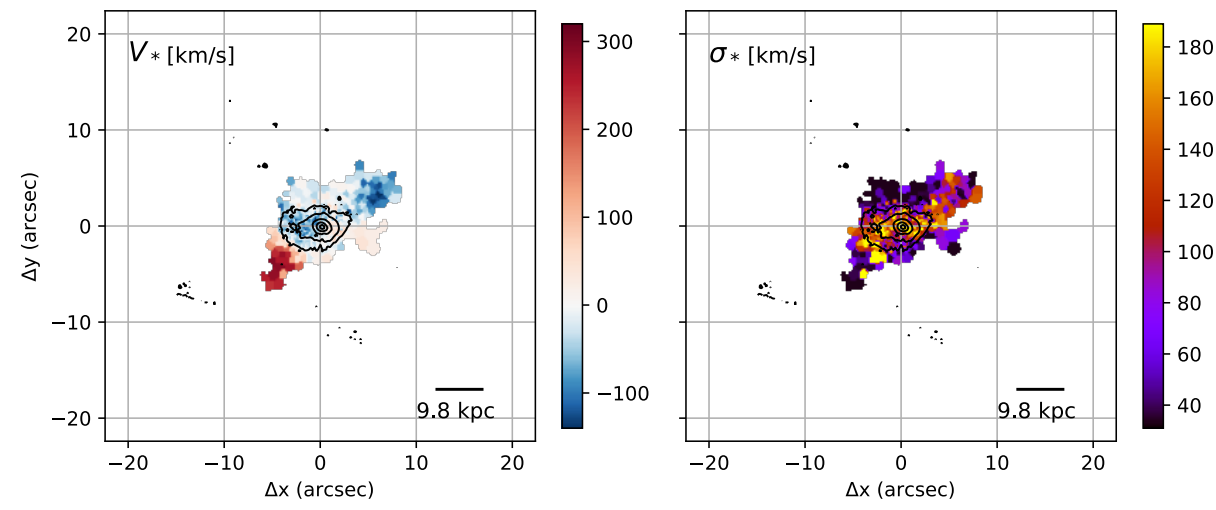

Fig. C.78. IRAS 20087-0308 stellar kinematic maps from the pPXF analysis with contours from HST/F160W. The left panel shows the stellar velocity $V_{*}$, and the right panel represents the velocity dispersion $\sigma_{*}$. 


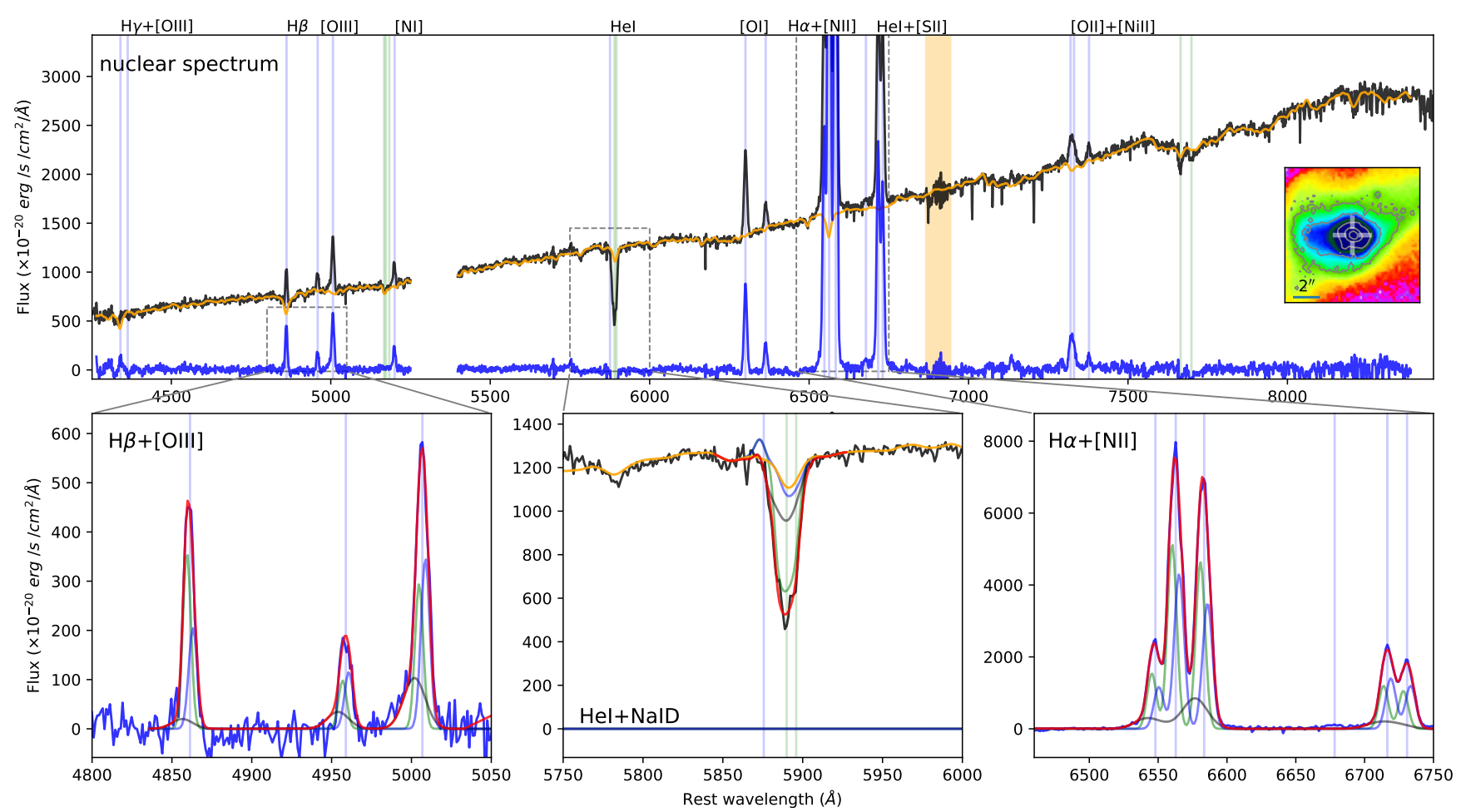

Fig. C.79. IRAS 20087-0308 nuclear spectrum extracted from a circular aperture with $r<0.4^{\prime \prime}$, with the corresponding pPXF (top panel) and multi-component (bottom insets) best-fit models. See Fig. C.4 for details. 

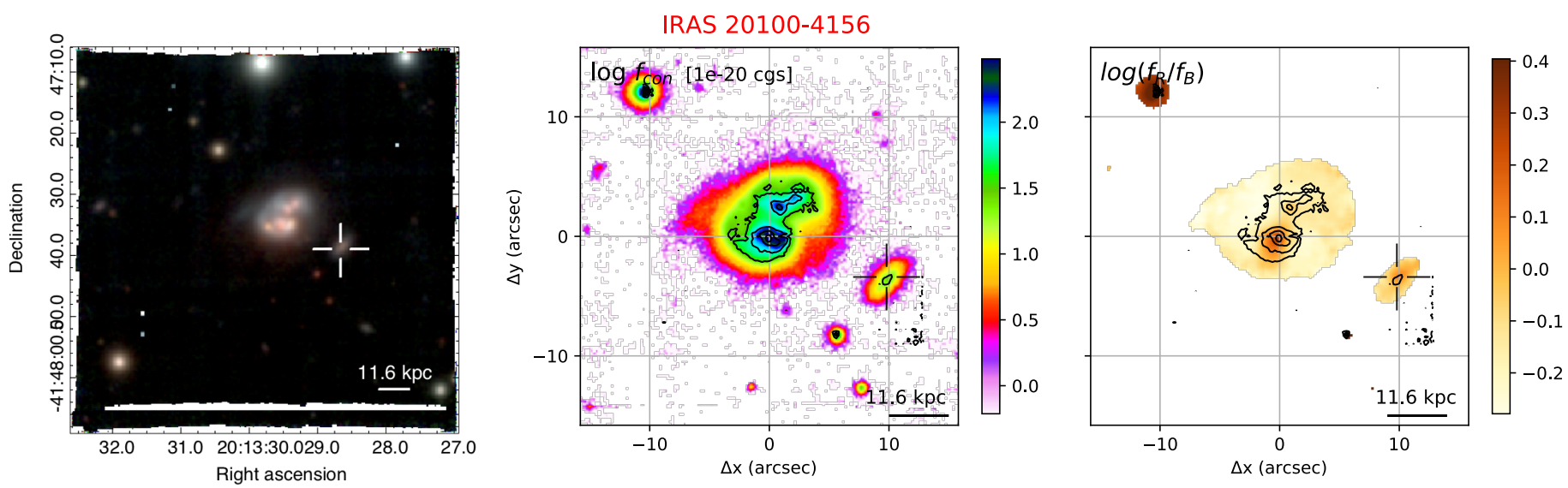

Fig. C.80. IRAS 20100-4156 images from MUSE observations with TOT = $2.04 \mathrm{hr}$. Left: Colour-composite optical image showing [O III] (green,

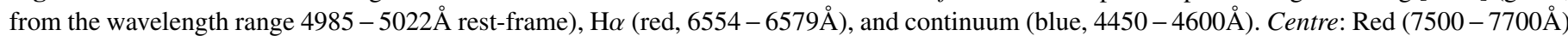
continuum image from MUSE with contours from HST/F160W. Right: Stellar continuum colour map obtained from MUSE by dividing the red continuum image (central panel) by a blue image obtained by collapsing the stellar emission in the range 4450-4600A ; contours from HST/F160W. In all panels, we display the IRAS 20100-4156 companion with a cross.
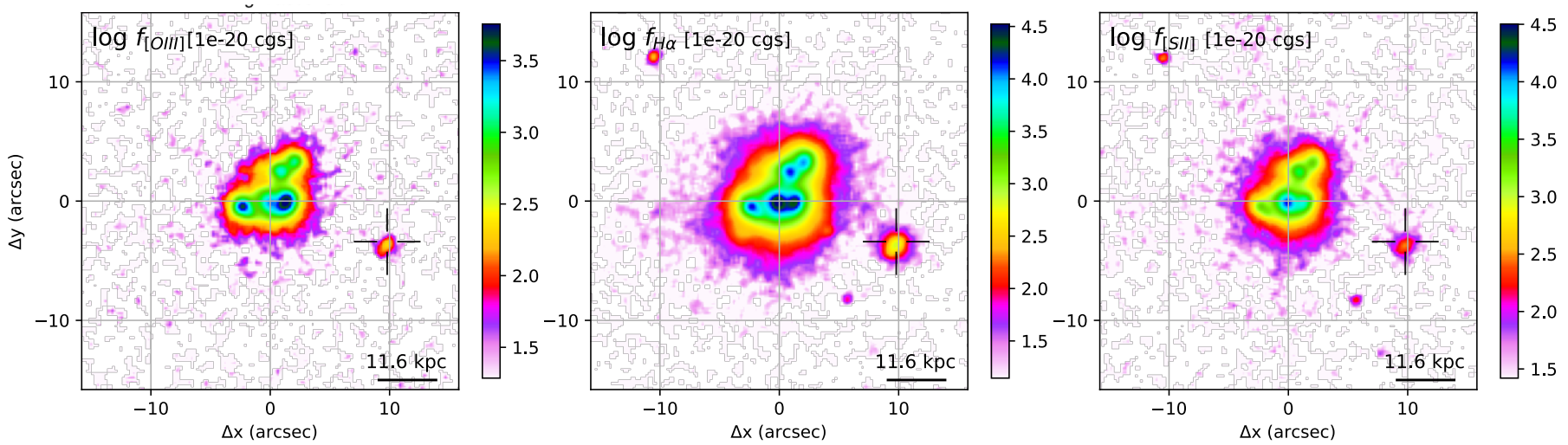

Fig. C.81. IRAS 20100-4156 emission line images from MUSE observations. [O III] (left, from the wavelength range $4985-5022 \AA$ rest-frame), $\mathrm{H} \alpha$ (centre, $6554-6579 \AA$ ), and [S II] (right, $6691-6743 \AA$ ) images have been obtained by subtracting continuum emission using the adjacent regions at shorter and longer wavelengths with respect to the emission line systemics. In all panels, we display the 20100-4156 companion with a cross.
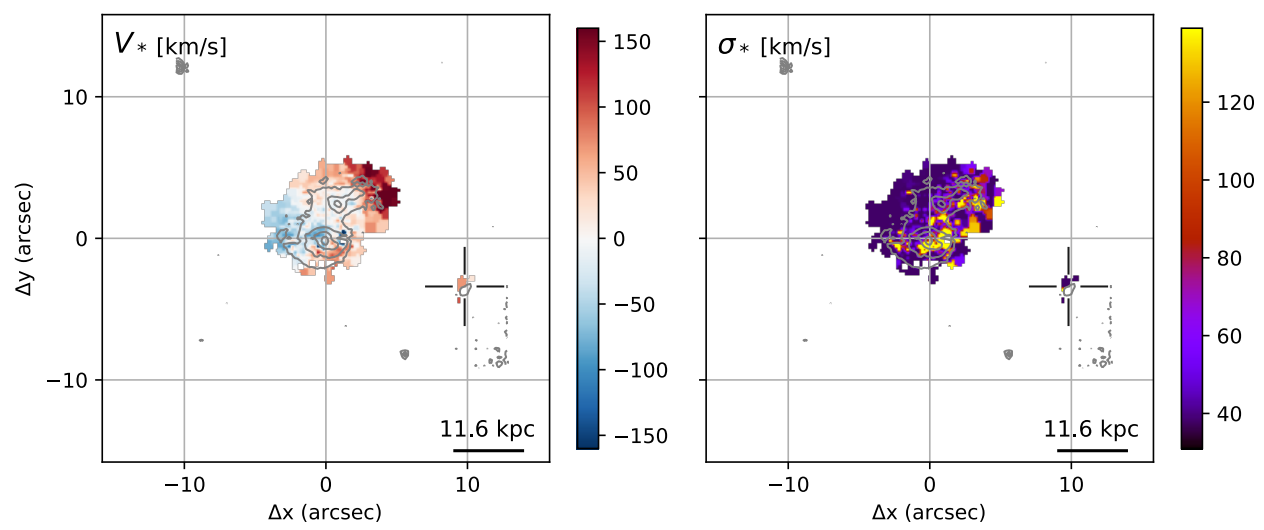

Fig. C.82. IRAS 20100-4156 stellar kinematic maps from the pPXF analysis with contours from HST/F160W. The left panel shows the stellar velocity $V_{*}$, and the right panel represents the velocity dispersion $\sigma_{*}$. 


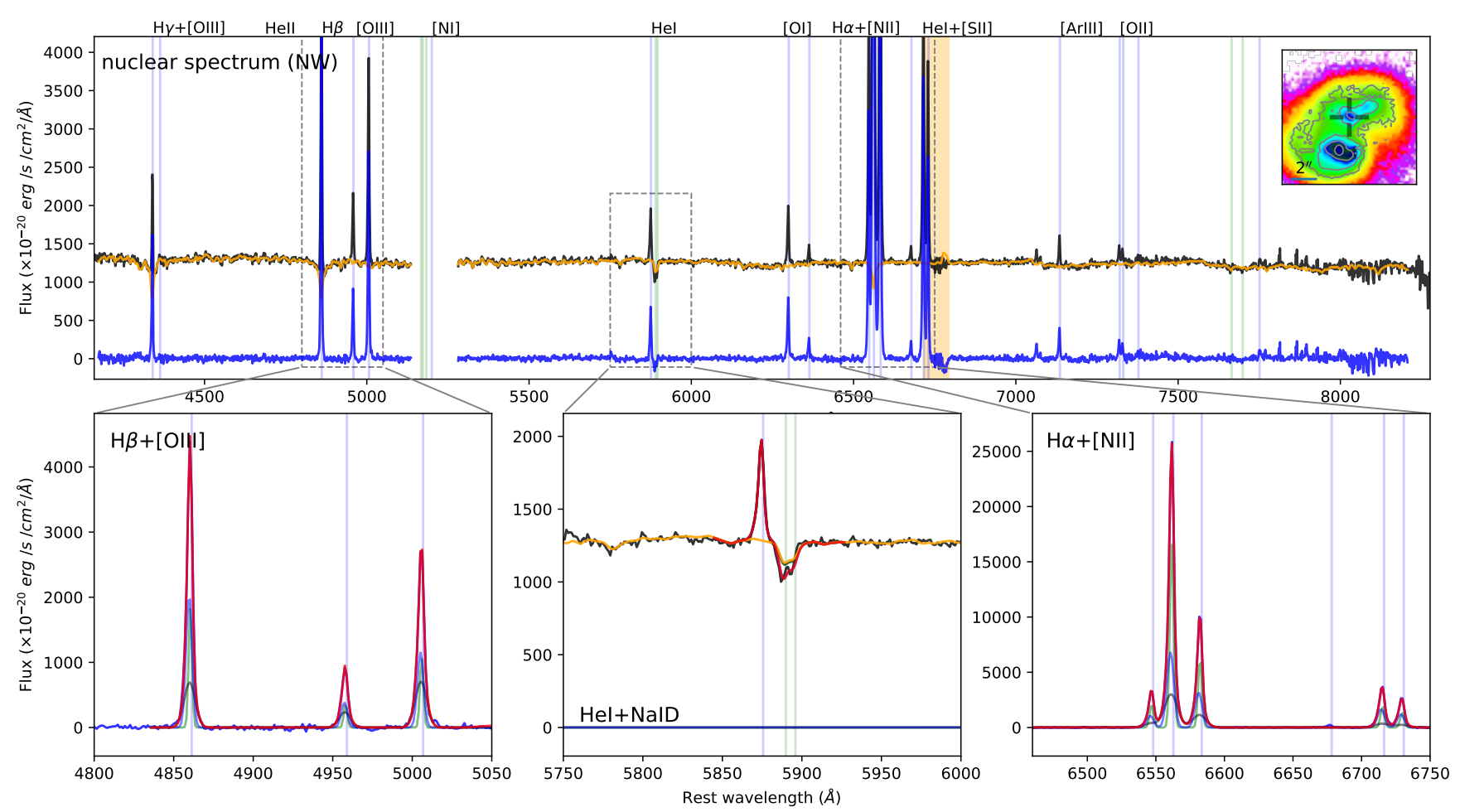

Fig. C.83. IRAS 20100-4156 NW nuclear spectrum extracted from a circular aperture with $r<0.4^{\prime \prime}$, with the corresponding pPXF (top panel) and multi-component (bottom insets) best-fit models. See Fig. C.4 for details.

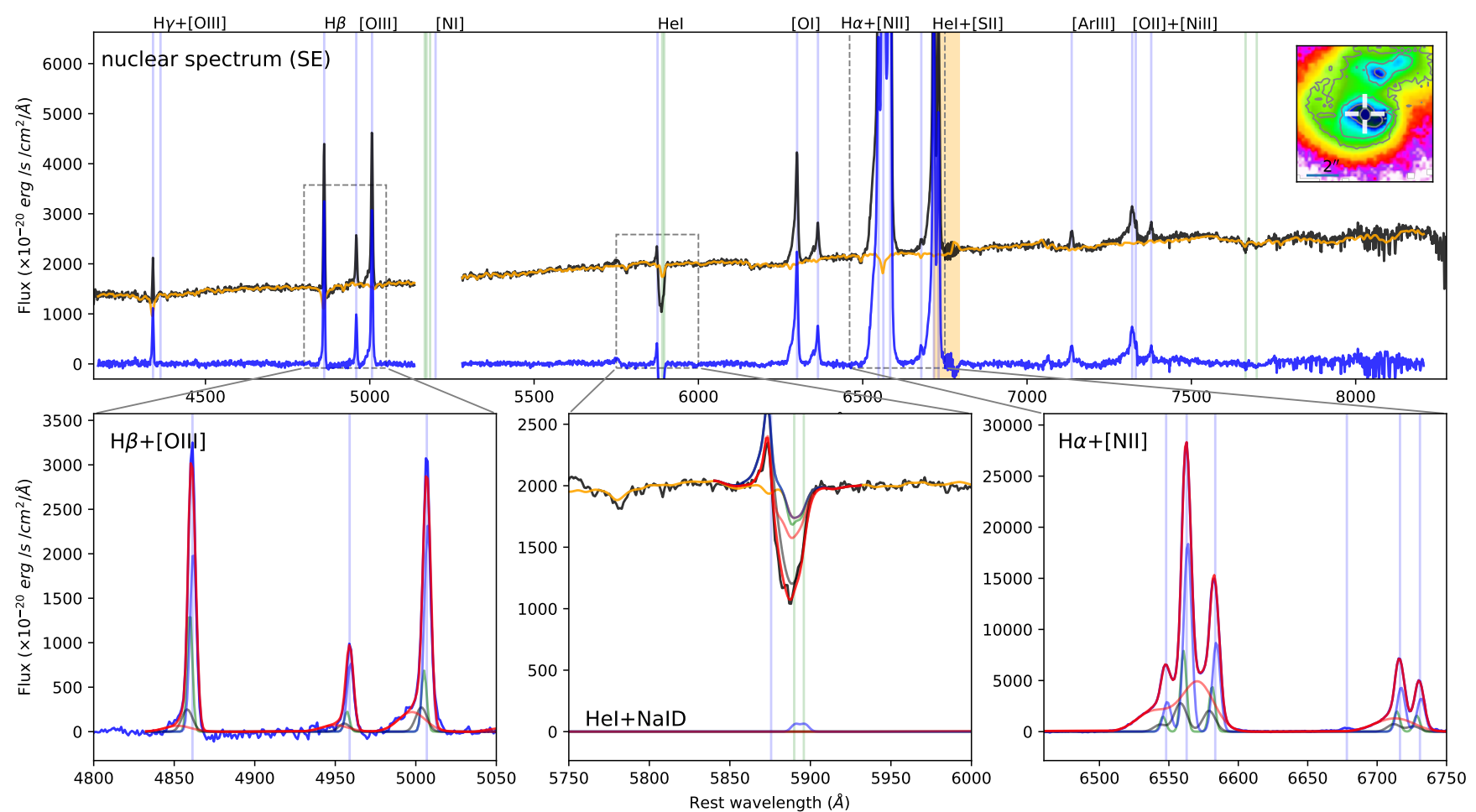

Fig. C.84. IRAS 20100-4156 SE nuclear spectrum extracted from a circular aperture with $r<0.4^{\prime \prime}$, with the corresponding pPXF (top panel) and multi-component (bottom insets) best-fit models. See Fig. C.4 for details. 

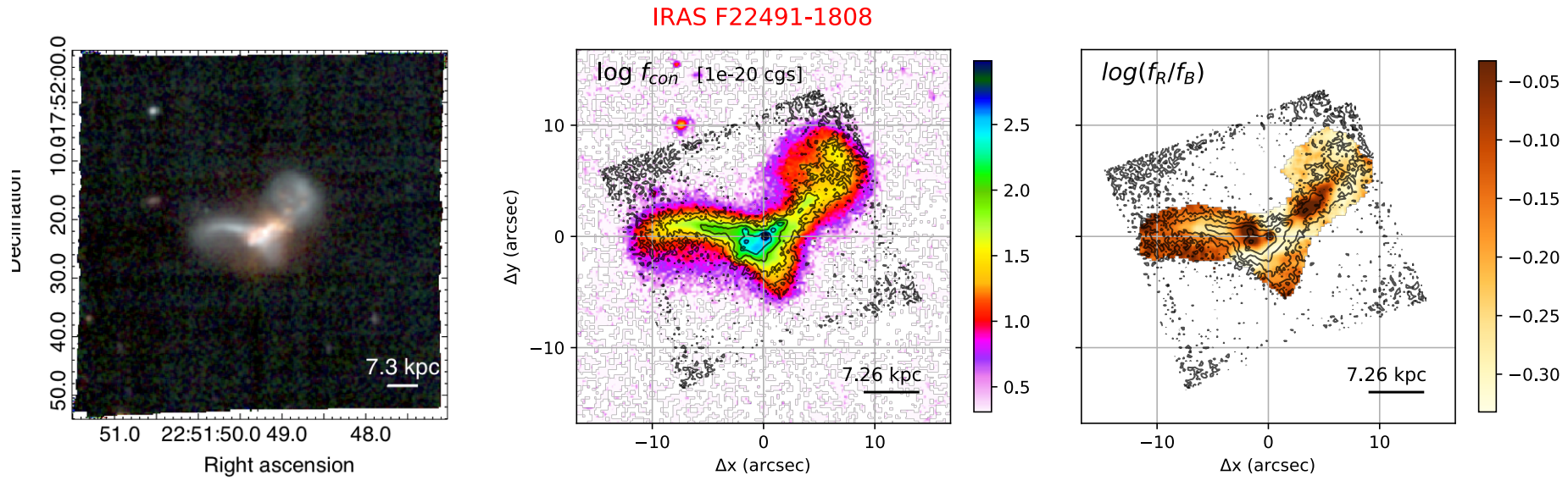

Fig. C.85. IRAS F22491-1808 images from MUSE observations with TOT $=0.41 \mathrm{hr}$. Left: Colour-composite optical image showing [O III] (green, from the wavelength range $4998-5017 \AA$ rest-frame), $\mathrm{H} \alpha$ (red, $6556-6575 \AA$ ), and stellar continuum (blue, $4460-4740 \AA$ ). Centre: Red $(8020-8120 \AA)$ stellar continuum image from MUSE with contours from HST/F160W. Right: Stellar continuum colour map obtained from MUSE by dividing the red continuum image (central panle) by a blue image obtained collapsing the stellar emission in the range $4460-4740 \AA$; contours from HST/F160W.
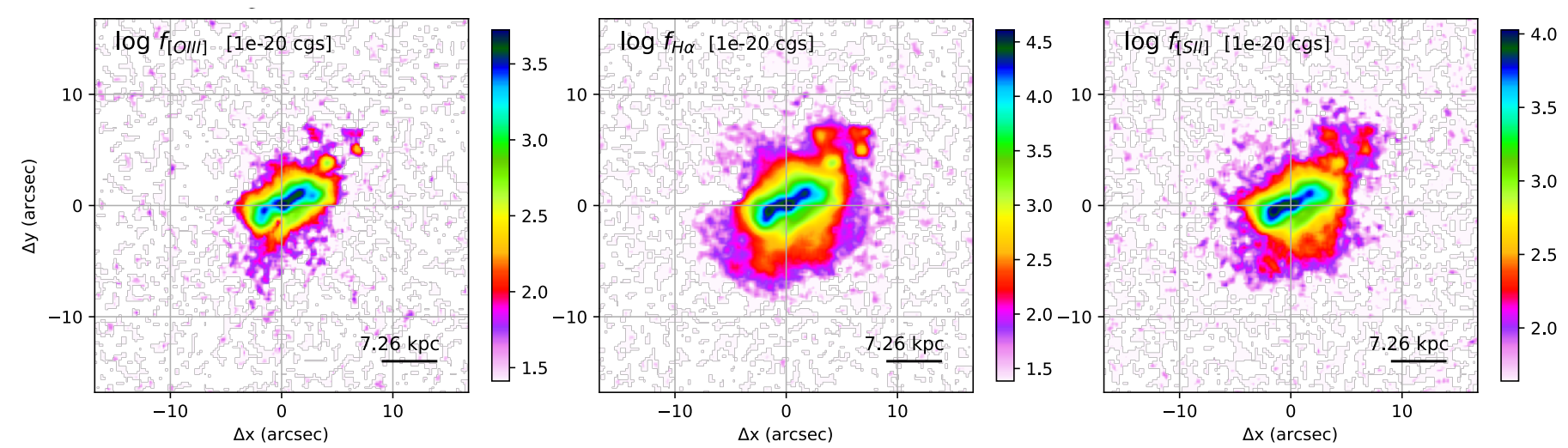

Fig. C.86. IRAS F22491-1808 emission line images from MUSE observations. [O III] (left, from the wavelength range 4998 - 5017 ̊̊ rest-frame), $\mathrm{H} \alpha$ (centre, $6556-6575 \AA$ ), and [S II] (right, $6707-6739 \AA$ ) images have been obtained by subtracting continuum emission using the adjacent regions at shorter and longer wavelengths with respect to the emission line systemics.
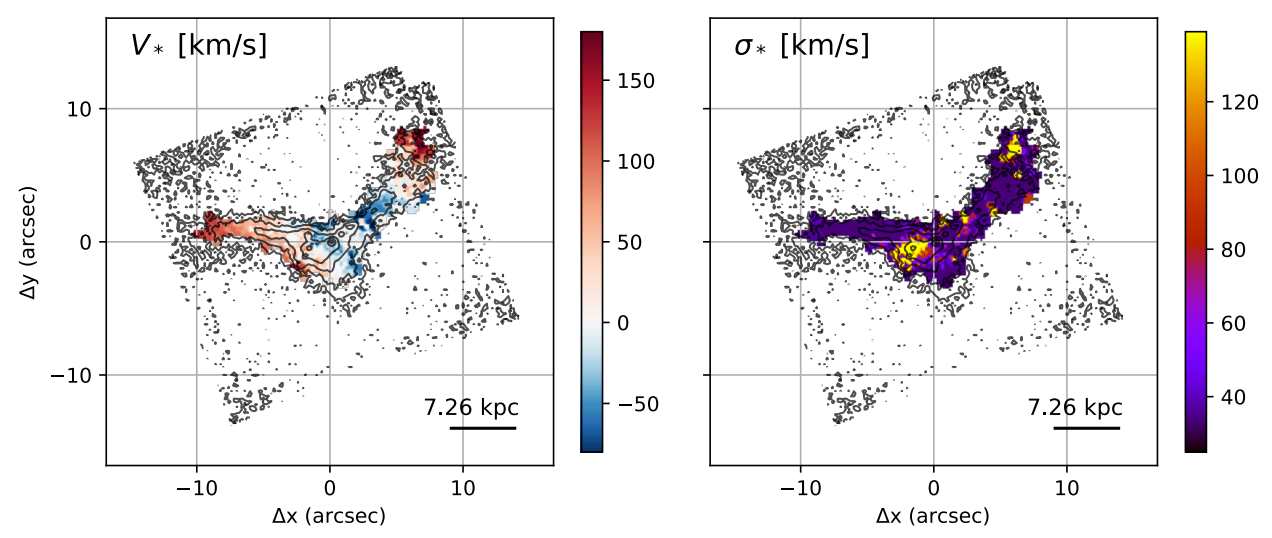

Fig. C.87. IRAS F22491-1808 stellar kinematic maps from the pPXF analysis with contours from HST/F160W. The left panel shows the stellar velocity $V_{*}$, and the right panel represents the velocity dispersion $\sigma_{*}$. 


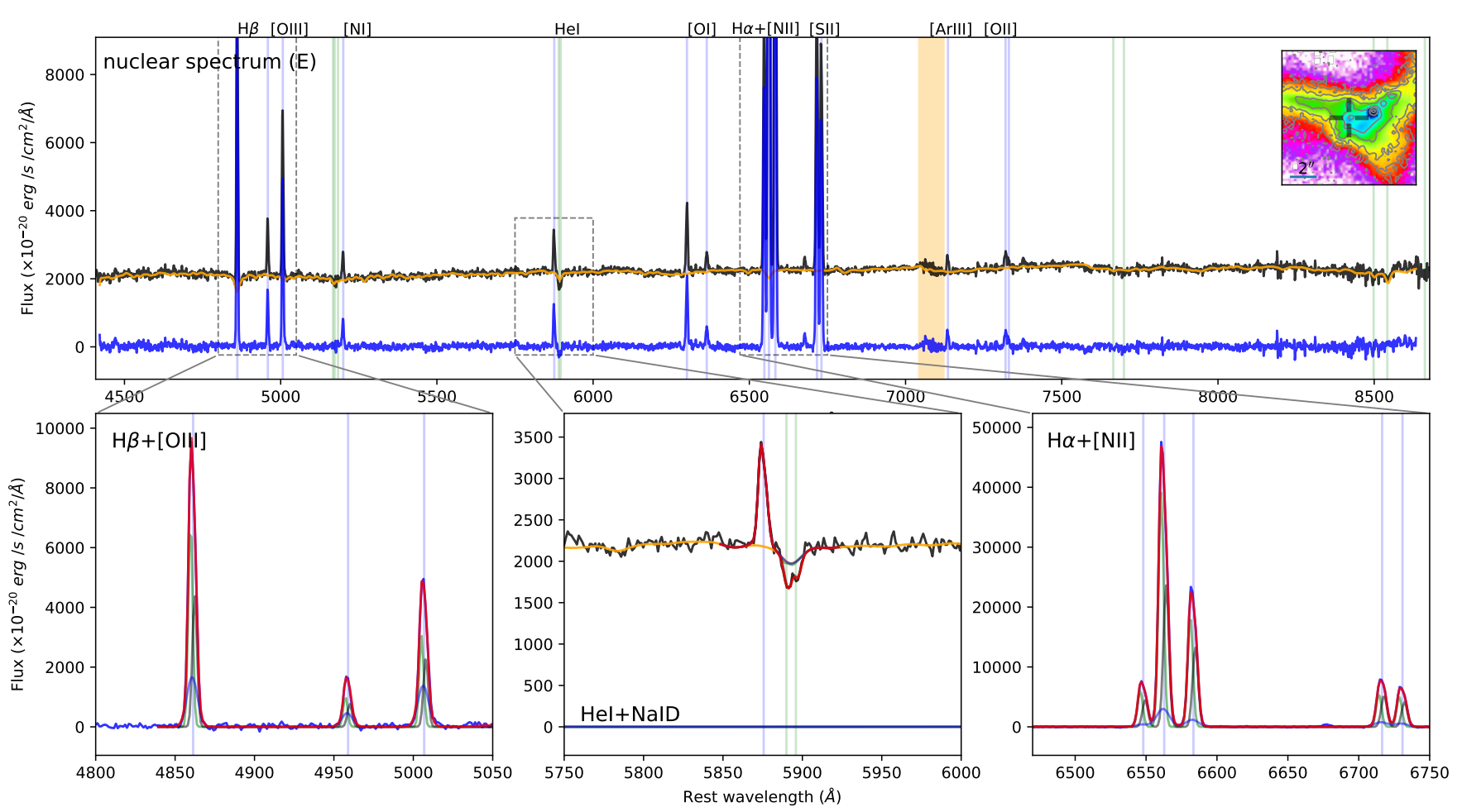

Fig. C.88. IRAS F22491-1808 E nuclear spectrum extracted from a circular aperture with $r<0.4^{\prime \prime}$, with the corresponding pPXF (top panel) and multi-component (bottom insets) best-fit models. See Fig. C.4 for details.
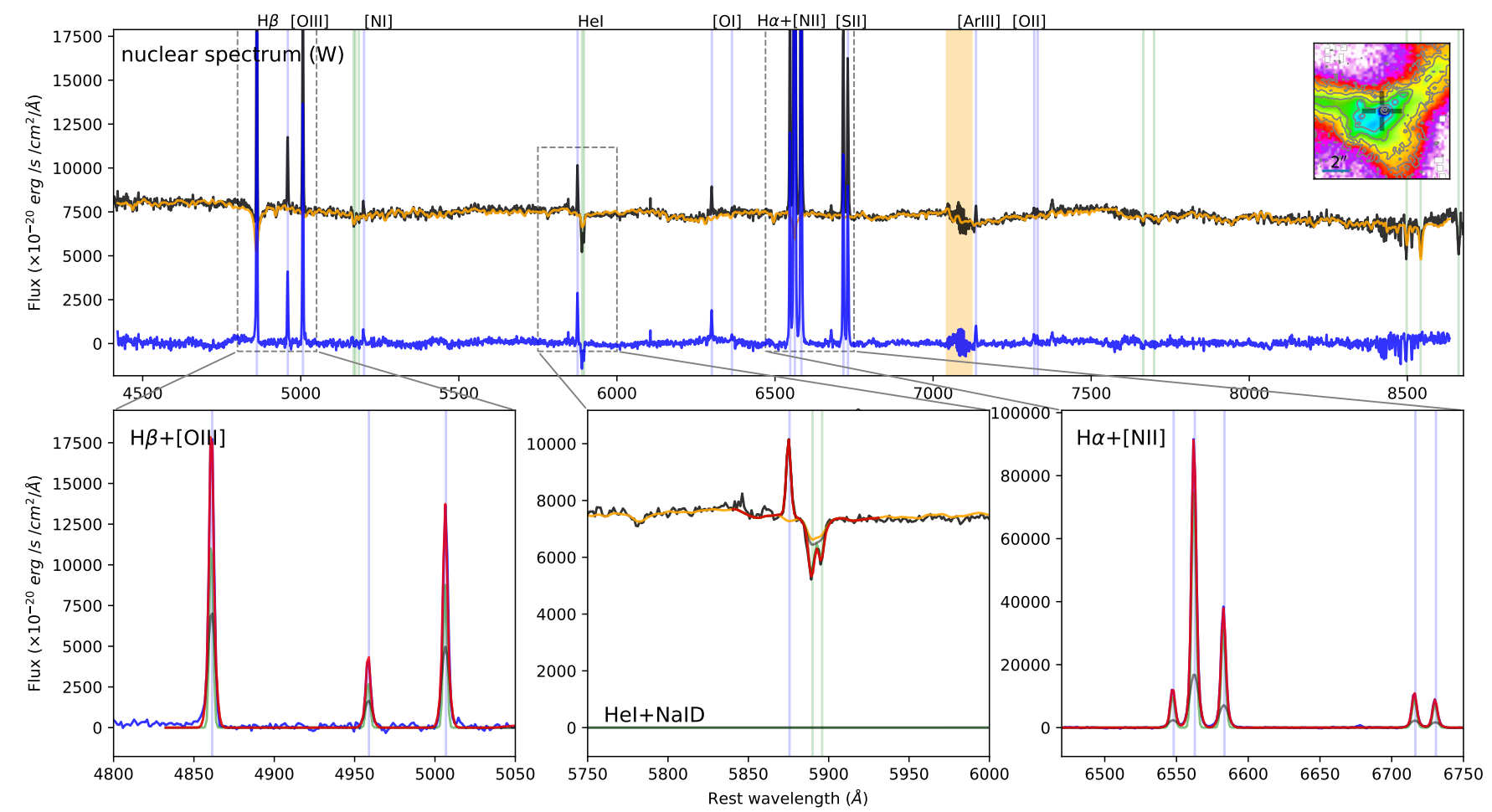

Fig. C.89. IRAS F22491-1808 W nuclear spectrum extracted from a circular aperture with $r<0.4^{\prime \prime}$, with the corresponding pPXF (top panel) and multi-component (bottom insets) best-fit models. See Fig. C.4 for details. 